2016

\title{
Modeling Spatially Varying Effects of Chemical Mixtures
}

Jenna Czarnota

Virginia Commonwealth University

Follow this and additional works at: https://scholarscompass.vcu.edu/etd

Part of the Biostatistics Commons

(C) The Author

\section{Downloaded from}

https://scholarscompass.vcu.edu/etd/4361

This Dissertation is brought to you for free and open access by the Graduate School at VCU Scholars Compass. It has been accepted for inclusion in Theses and Dissertations by an authorized administrator of VCU Scholars Compass. For more information, please contact libcompass@vcu.edu. 
Modeling Spatially Varying Effects of Chemical Mixtures

A dissertation submitted in partial fulfillment of the requirements for the degree of Doctor of Philosophy at Virginia Commonwealth University.

\section{by}

\section{Jenna Nichole Czarnota}

B.S. in Mathematics, University of Maryland Baltimore County, 2010

Director: David C. Wheeler

Assistant Professor, Department of Biostatistics

Virginia Commonwealth University

Richmond, Virginia

May, 2016 


\section{Acknowledgment}

I would like to extend my sincerest gratitude to my family and friends for their constant love and support. I am also tremendously grateful for the support and guidance of my advisors and for their unfailing provision of insight and encouragement. 


\section{Table of Contents}

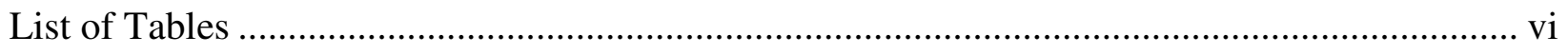

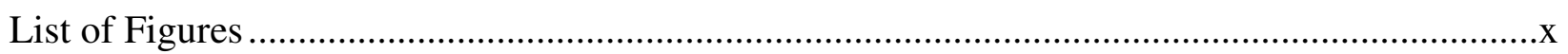

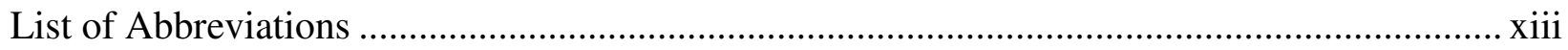

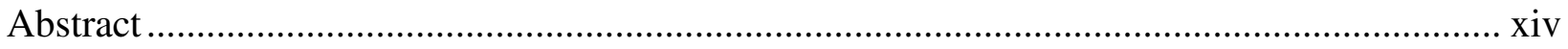

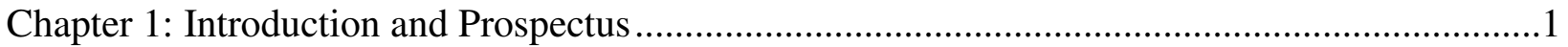

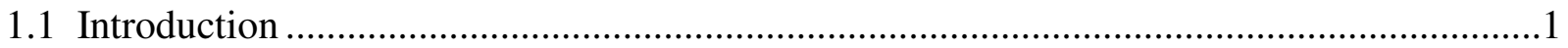

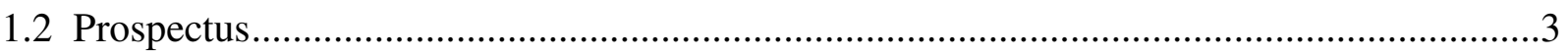

Chapter 2: Analysis of Environmental Chemical Mixtures and Non-Hodgkin Lymphoma Risk in

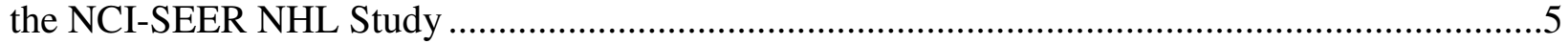

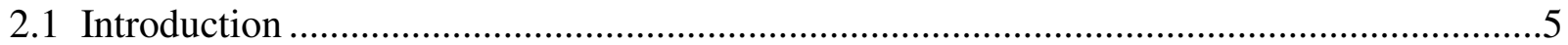

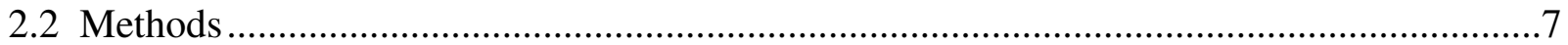

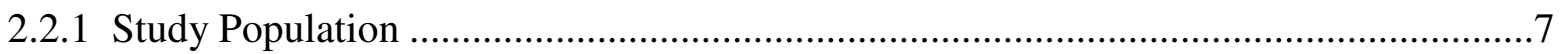

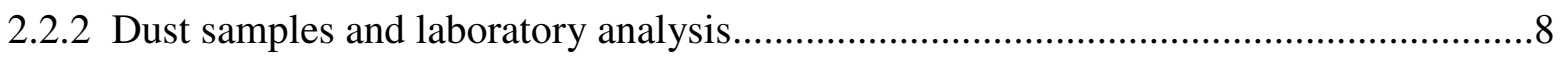

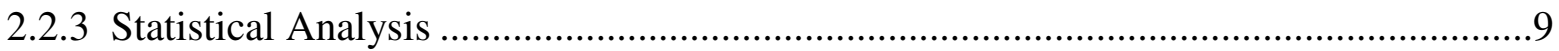

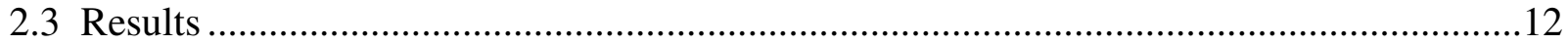

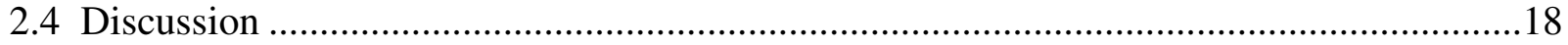

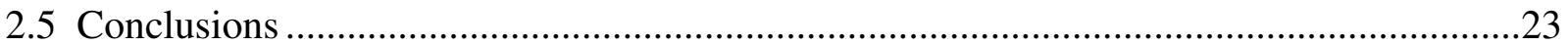

Chapter 3: Assessment of Weighted Quantile Sum Regression for Modeling Chemical Mixtures

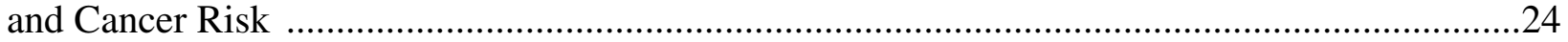

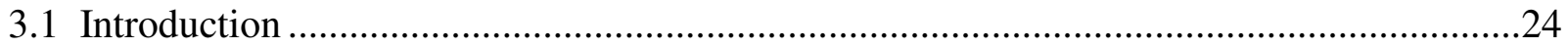

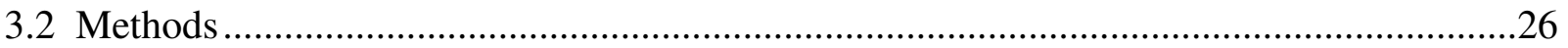

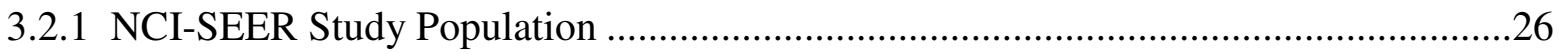

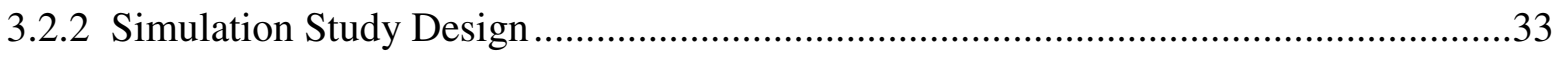

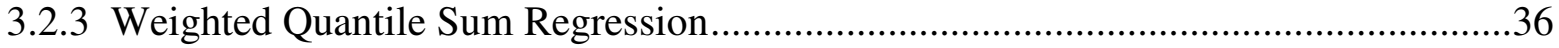

3.2.4 Comparison of WQS regression with lasso, adaptive lasso, and elastic net ...............38

3.3 Results .39 


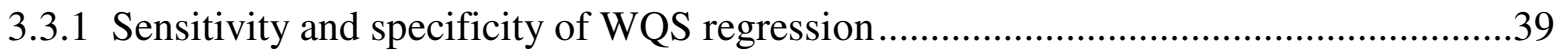

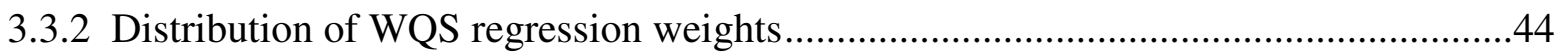

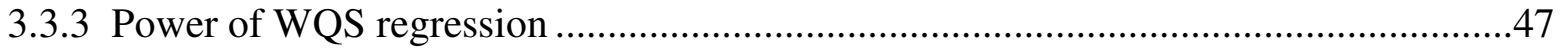

3.3.4 Comparison of WQS regression with lasso, adaptive lasso, and elastic net ................49

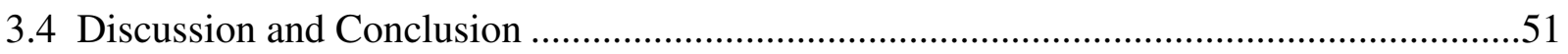

Chapter 4: Evaluating Geographically Weighted Regression Models for Environmental Chemical Risk Analysis ............................................................................................................5

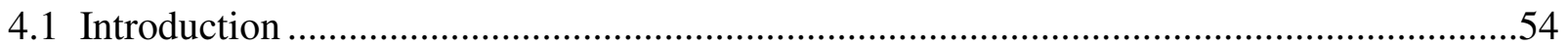

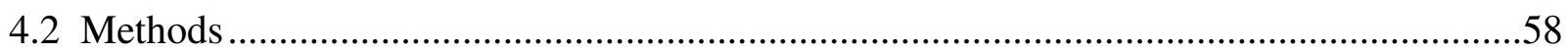

4.2.1 Simulating spatially varying exposure and dose-dependent association with an

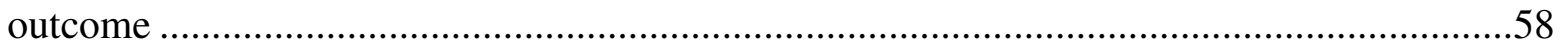

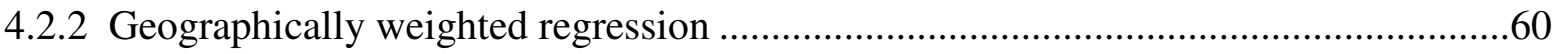

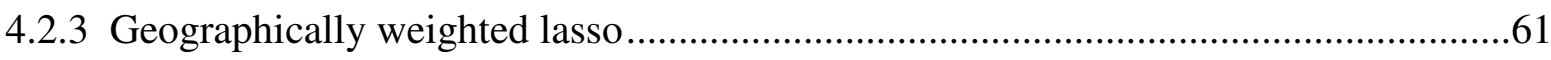

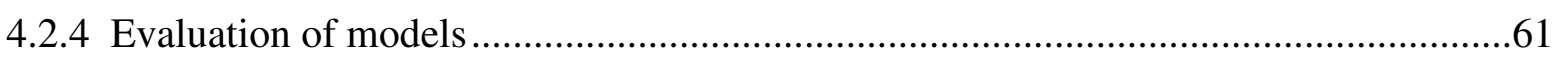

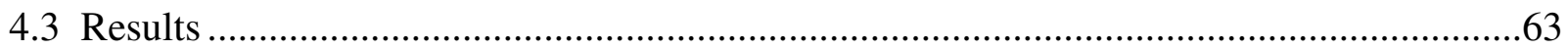

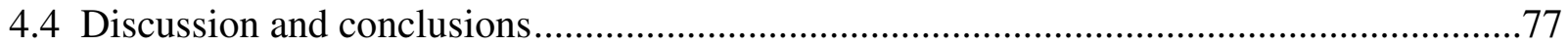

Chapter 5: Local Weighted Quantile Sum Regression ...............................................................81

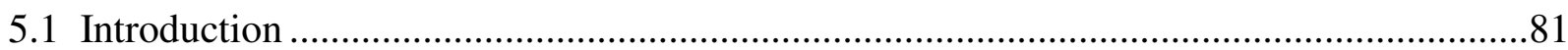

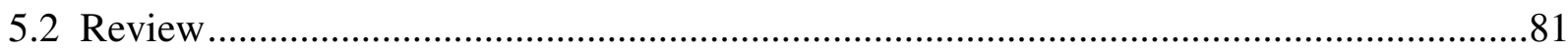

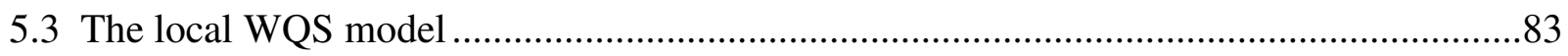

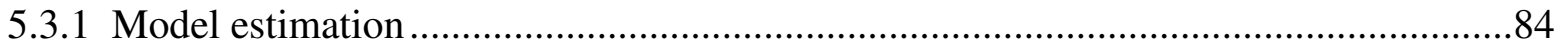

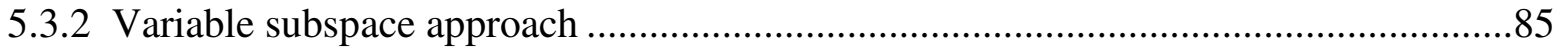

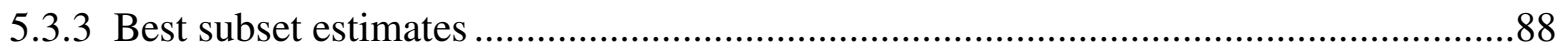

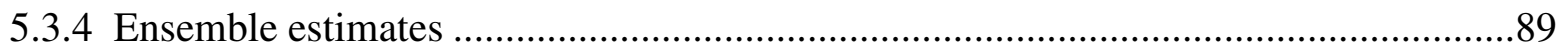

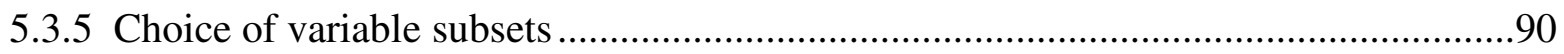

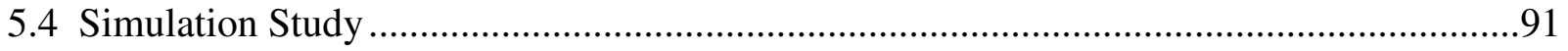

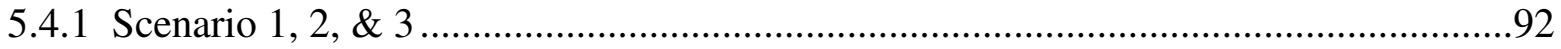

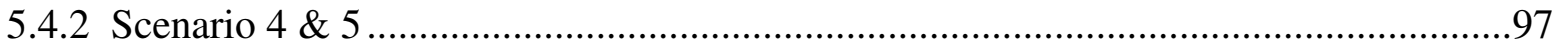

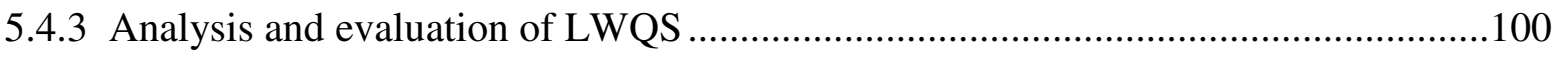

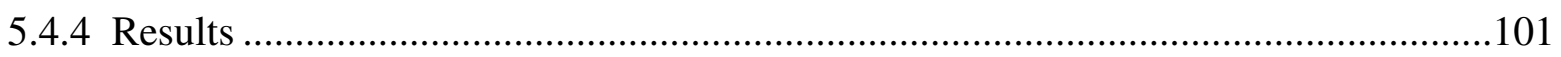

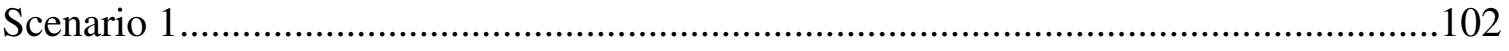

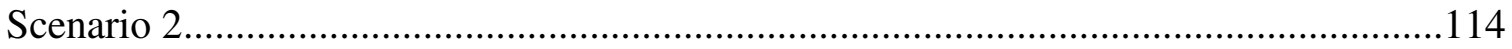

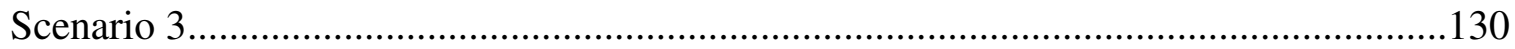




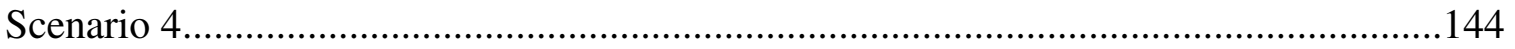

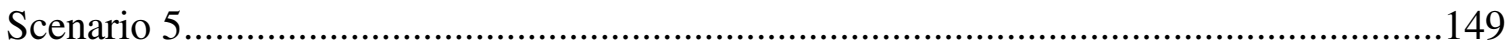

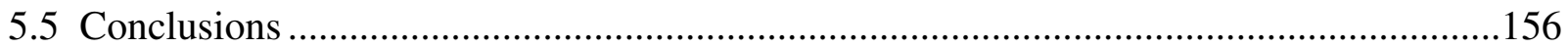

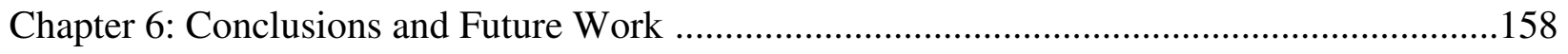

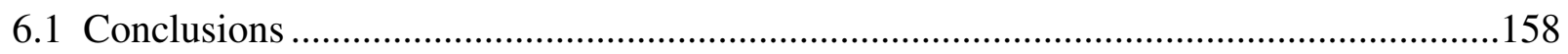

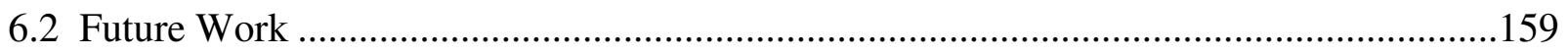

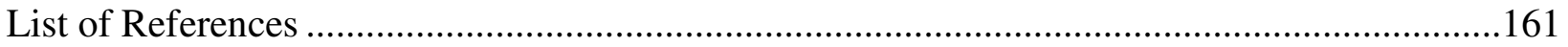

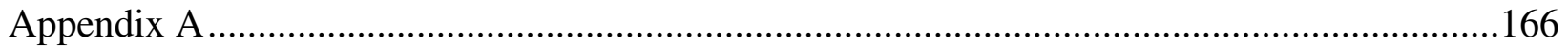

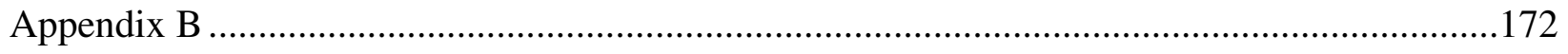

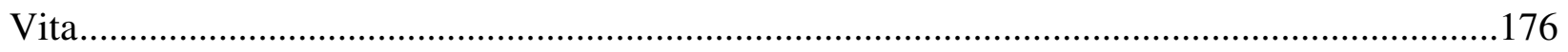




\section{List of Tables}

2.1: Characteristics of the NCI-SEER NHL study population overall and by study site .13

2.2: Associations between non-Hodgkin lymphoma and the weighted quantile sum regression index in the study population and in each study site

2.3: Weighted quantile sum regression index weights estimated in the study population and in each study site

2.4: Associations between non-Hodgkin lymphoma subtypes and the weighted quantile sum regression index in the study population

3.1: Correlations within chemical group by study site and across the full population in the NCI-SEER NHL study

3.2: Median $[\mathrm{IQR}]$ number of correctly chosen chemicals for the five WQS indices.

3.3: Median $[\mathrm{IQR}]$ number of incorrectly chosen chemicals for the five WQS indices.....

3.4: Summary of testing results for the five WQS indices across 100 simulated examples for the continuous outcome variable

3.5: Summary of testing results for the five WQS indices across 100 simulated examples for the binary outcome variable.

3.6: Median $[I Q R]$ number of correctly selected chemicals for lasso, adaptive lasso, elastic net, and WQS regression for the full study population.

3.7: Median [IQR] number of incorrectly selected chemicals for lasso, adaptive lasso, elastic net, and WQS regression for the full study population.

4.1: Average predictor and response values across the 100 simulated data sets for the cases of independent chemicals (case 1) and correlated chemicals (case 2).....

4.2: Median (IQR) of summary statistics for GWR and GWL models across the 100 simulated data sets for the cases of independent chemicals (case 1) and correlated chemicals (case 2) .......65

4.3: Median (IQR) percentage of GWR and GWL coefficient estimates that were positive, negative, and zero across the 100 simulated data sets for the cases of independent chemicals (case 1) and correlated chemicals (case 2). 
4.4: Median (IQR) percentage of GWR coefficient estimates that were positive, negative, and zero across the 100 simulated data sets when considering \pm 1 and \pm 2 standard errors of regression coefficient estimates for the cases of independent chemicals (case 1) and correlated chemicals (case 2)

5.1: Simulated mean exposure concentrations $(\mathrm{SD}=0.5)$ for the six exposure variables in scenario 1,2 , and 3

5.2: Environmental correlation structure 1 - Correlation among the observed exposures of three PCBs (PCB 153, PCB 170, PCB 180), two PAHs (Benzo(a)pyrene, Chrysene), and one pesticide (DDT) in the NCI-SEER NHL study

5.3: Environmental correlation structure 2 - Correlation among the observed exposures of three PCBs (PCB 153, PCB 170, PCB 180), one pesticide (DDT), and two PAHs (Benzo(a)pyrene, Chrysene) in the NCI-SEER NHL study

5.4: Environmental correlation structure 3 - Correlation among the observed exposures of six PAHs (Benzo(a)pyrene, Benzo(b)fluorene, Benzo(k)fluorene, Chrysene, dibenz(ah)anthracene, idenopyrene) in the NCI-SEER NHL study.

5.5: Simulated LWQS parameters for scenario 1

5.6: Summary of model AICc by variable subset and the corresponding number of times each candidate bandwidth value $\gamma$ was chosen over the 100 simulated data sets for scenario 1 in the case of a) independent exposures.

5.7: Summary of model AICc by variable subset and the corresponding number of times each candidate bandwidth value $\gamma$ was chosen over the 100 simulated data sets for scenario 1 in the case of $b) r(x 1, x 3)=0.6$.

5.8: Summary of model AICc by variable subset and the corresponding number of times each candidate bandwidth value $\gamma$ was chosen over the 100 simulated data sets for scenario 1 in the case of c) environmental correlation structure 1

5.9: Summary of model AICc in simulation scenario 1 for the best subset method and for ensemble methods using variable subset sizes of 3, 4, and 5 for the cases of a) independent exposures, b) $\mathrm{r}(\mathrm{x} 1, \mathrm{x} 3)=0.6$, and c) environmental correlation structure 1 ......

5.10: Summary of the LWQS parameter estimates for simulation scenario 1 using the best subset and ensemble methods for the cases of a) independent exposures, b) $r(x 1, x 3)=0.6$, and c) environmental correlation structure 1

5.11: Summary of the bias and RMSE in the estimated LWQS parameters for simulation scenario 1 using the best subset and ensemble methods for the cases of a) independent exposures, b) $\mathrm{r}(\mathrm{x} 1, \mathrm{x} 3)=0.6, \mathrm{c})$ environmental correlation structure 1 109

5.12: Simulated LWQS parameters for scenario 2 .114 
5.13: Summary of model AICc by variable subset and the corresponding number of times each candidate bandwidth value $\gamma$ was chosen over the 100 simulated data sets for scenario 2 in the case of a) independent exposures.

5.14: Summary of model AICc by variable subset and the corresponding number of times each candidate bandwidth value $\gamma$ was chosen over the 100 simulated data sets for scenario 2 in the case of $b) r(x 1, x 3)=0.6$.

5.15: Summary of model AICc by variable subset and the corresponding number of times each candidate bandwidth value $\gamma$ was chosen over the 100 simulated data sets for scenario 2 in the case of c) environmental correlation structure 2

5.16: Summary of model AICc in simulation scenario 2 for the best subset method and for ensemble methods using variable subset sizes of 3,4 , and 5 for the cases of a) independent exposures, $\mathrm{b}) \mathrm{r}(\mathrm{x} 1, \mathrm{x} 3)=0.6$, and $\mathrm{c}$ ) environmental correlation structure 2

5.17: Summary of the LWQS parameter estimates for simulation scenario 2 using the best subset and ensemble methods for the cases of a) independent exposures, b) $r(x 1, x 3)=0.6$, and c) environmental correlation structure 2

5.18: Summary of the bias and RMSE in the estimated LWQS parameters for simulation scenario 2 using the best subset and ensemble methods for the cases of a) independent exposures, b) $\mathrm{r}(\mathrm{x} 1, \mathrm{x} 3)=0.6, \mathrm{c})$ environmental correlation structure 2

5.19: Simulated LWQS parameters for scenario 3

5.20: Summary of model AICc by variable subset and the corresponding number of times each candidate bandwidth value $\gamma$ was chosen over the 100 simulated data sets for scenario 3 in case a) environmental correlation structure 2

5.21: Summary of model AICc by variable subset and the corresponding number of times each candidate bandwidth value $\gamma$ was chosen over the 100 simulated data sets for scenario 3 in case b) environmental correlation structure 3

5.22: Summary of model AICc in simulation scenario 3 for the best subset method and for ensemble methods using variable subset sizes of 3,4 , and 5 for the cases of a) environmental correlation matrix 2 and $b$ ) environmental correlation matrix 3 .

5.23: Summary of the LWQS parameter estimates for simulation scenario 3 using the best subset and ensemble methods in the cases of a) environmental correlation matrix 2 and b) environmental correlation matrix 3 .

5.24: Summary of the bias and RMSE in the estimated LWQS parameters for simulation scenario 3 using the best subset and ensemble methods for the cases of a) environmental correlation matrix 2 and b) environmental correlation matrix 3 ...... 
5.25: Summary of model AICc by variable subset and the corresponding number of times each candidate bandwidth value $\gamma$ was chosen over the 100 simulated data sets for scenario 4 ........144

5.26: Summary of model AICc in simulation scenario 4 for the best subset method and for ensemble methods using variable subset sizes of 3,4 , and 5 .

5.27: Summary of the LWQS parameter estimates for simulation scenario 4 using the best subset and ensemble methods

5.28: Summary of the bias and RMSE in the estimated LWQS parameters for simulation scenario 4 using the best subset and ensemble methods

5.29: Summary of model AICc by variable subset and the corresponding number of times each candidate bandwidth value $\gamma$ was chosen over the 100 simulated data sets for scenario 5 ........150

5.30: Summary of model AICc in simulation scenario 5 for the best subset method and for ensemble methods using variable subset sizes of 3,4 , and 5

5.31: Summary of the LWQS parameter estimates for simulation scenario 5 using the best subset and ensemble methods

5.32: Summary of the bias and RMSE in the estimated LWQS parameters for simulation scenario 5 using the best subset and ensemble methods 


\section{List of Figures}

3.1: Distribution of chemical concentrations in the NCI-SEER NHL study for a) PCBs, b) PAHs, and c) Pesticides/Insecticides

3.2: Distribution of Pearson pairwise correlation coefficients among chemical concentrations (on the log scale) by study site in the NCI-SEER NHL study

3.3: Distribution of the observed intergroup correlations for PCBs, PAHs, and pesticides by study site and across the full study population

3.4: Modified receiver operating curves for the WQS index derived from the full study population with varying weight thresholds for chemical selection

3.5: Distribution of WQS index weights for the five WQS indices across the 100 simulated data sets for the seven chemicals weakly associated $(r=0.1)$ with the continuous outcome for a) moderate correlation ( $65 \%$ of that observed) among chemicals, and b) correlation among chemicals diminished to $1 \%$ of that observed..

4.1: Standardized partial regression coefficients in a multiple regression model with two variables

4.2: Plots of average simulated concentration values across 100 simulated data sets over a square study area for two scenarios: independent chemicals (case 1) and correlated chemicals (case 2)

4.3: Pairwise plots of average regression coefficients across the 100 simulated data sets for the cases of independent chemicals (case 1) and correlated chemicals (case 2) for GWR and GWL

4.4: Boxplots of average GWR and GWL regression coefficients across 100 simulated datasets for the two study regions for the cases of independent chemicals (case 1) and correlated chemicals (case 2 ).

4.5: Average GWR and GWL regression coefficient estimates over 100 simulated datasets for the cases of independent chemicals (case1) and correlated chemicals (case 2)

4.6: Estimated regression coefficients from GWR and GWL for one simulation of data under the cases of independent chemicals (case 1) and correlated chemicals (case 2) .76

5.1: Simulated mean exposure concentrations for scenario 1,2 , and 3 
5.2: Simulated parameter values over the study region for scenario 1 .95

5.3: Simulated parameter values over the study region for scenario 2 .96

5.4: Simulated parameter values over the study region for scenario 3 .97

5.5: Simulated exposure concentrations for scenario 4 and 5 . .98

5.6: Simulated parameter values over the study region for scenario 4 . .99 5.7: Simulated parameter values over the study region for scenario 5 100

5.8: Maps of the LWQS best subset parameter estimates in simulation scenario 1 for the cases of: a) independent exposures (panel 2); b) $\mathrm{r}(\mathrm{x} 1, \mathrm{x} 3)=0.6$ (panel 3), and c) environmental correlation matrix 1 (panel 4). The simulated LWQS parameters for scenario 1 are included for the purpose of comparison (panel 1)

5.9: Maps of the LWQS ensemble parameter estimates from subsets of size 5 in simulation scenario 1 for the correlation cases of: a) independent exposures (panel 2); b) $r(x 1, x 3)=0.6$ (panel 3), and c) environmental correlation matrix 1 (panel 4). The scenario 1 simulated LWQS parameters (panel 1) are included for the purpose of comparison.

5.10: Bias in ensemble parameter estimates from subsets of size 5 for scenario 1 in the cases of a) independent exposures (panel 1), b) r(x1, x3) = 0.6 (panel 2), and c) environmental correlation structure 1 (panel 3)

5.11: Maps of the LWQS best subset parameter estimates in simulation scenario 2 for the cases of: a) independent exposures (panel 2); b) r(x1,x3) = 0.6 (panel 3), and c) environmental correlation matrix 2 (panel 4). The simulated LWQS parameters for scenario 2 are included for the purpose of comparison (panel 1).

5.12: Bias in the best subset parameter estimates for scenario 2 in the cases of a) independent exposures (panel 1), b) $\mathrm{r}(\mathrm{x} 1, \mathrm{x} 3)=0.6$ (panel 2), and c) environmental correlation structure 2 (panel 3)

5.13: Maps of the LWQS ensemble parameter estimates from subsets of size 5 for scenario 2 in the cases of a) independent exposures (panel 2), b) $r(x 1, x 3)=0.6$ (panel 3), and c) environmental correlation matrix 2 (panel 4). The scenario 2 simulated LWQS parameters (panel 1) are included for the purpose of comparison.

5.14: Bias in ensemble parameter estimates from subsets of size 5 for scenario 2 in the cases of a) independent exposures (panel 1), b) r(x1, x3) = 0.6 (panel 2), and c) environmental correlation structure 2 (panel 3)

5.15: Maps of the LWQS best subset parameter estimates in simulation scenario 3 for cases a) environmental correlation matrix 2 (panel 2), and b) environmental correlation matrix 3 (panel 3 ). The simulated LWQS parameters for scenario 3 are included for the purpose of comparison (panel 1). 
5.16: Bias in the best subset parameter estimates in scenario 3 for the cases of a) environmental correlation matrix 2 (panel 1) and b) environmental correlation matrix 3 (panel 2)

5.17: Maps of the ensemble parameter estimates in simulation scenario 3 for cases a) environmental correlation matrix 2 (panel 2) and b) environmental correlation matrix 3 (panel 3)

5.18: Bias in ensemble parameter estimates from subsets of size 5 in scenario 3 for the cases of a) environmental correlation matrix 2 (panel 1) and b) environmental correlation matrix 3 (panel 2).

5.19: Maps of the simulated LWQS parameters (panel 1), best subset estimates (panel 2), and ensemble estimates from subsets of size 4 (panel 3) in simulation scenario 4

5.20: Bias in the best subset estimates (panel 1) and ensemble estimates from subsets of size 4 (panel 2) in simulation scenario 4 .

5.21: Maps of the simulated LWQS parameters (panel 1), best subset estimates (panel 2), and ensemble estimates from subsets of size 4 (panel 3) in simulation scenario 5 .

5.22: Bias in the best subset estimates (panel 1) and ensemble estimates from subsets of size 4 (panel 2) in simulation scenario 5 . 


\title{
List of Abbreviations
}

\author{
2,4-D 2,4-dichlorophenoxyacetic acid \\ AIC Akaike Information Criterion \\ AICc corrected Akaike Information Criterion \\ CI confidence interval \\ CLL chronic lymphocytic leukemia \\ DDE dichlorodiphenyldichloroethylene \\ DDT dichlorodiphenyltrichloroethane \\ DLBCL diffuse large B-cell lymphoma \\ GC/MS gas chromatography/mass spectrometry \\ GWL geographically weighted lasso \\ GWR geographically weighted regression \\ IQR interquartile range \\ LARS least angle regression \\ LWQS local weighted quantile sum \\ NCI-SEER National Cancer Institute - Surveillance, Epidemiology, and End Results Program \\ NHANES National Health and Nutrition Examination Survey \\ NHL non-Hodgkin lymphoma \\ NOS not otherwise specified \\ OR odds ratio \\ PAH polycyclic aromatic hydrocarbon \\ PCB polychlorinated biphenyl \\ RMSE root mean square error \\ RMSPE root mean square prediction error \\ SD standard deviation \\ SE standard error \\ SLL small lymphocytic leukemia \\ WQS weighted quantile sum
}




\begin{abstract}
MODELING SPATIALLY VARYING EFFECTS OF CHEMICAL MIXTURES

By Jenna N. Czarnota, Ph.D.

A dissertation submitted in partial fulfillment of the requirements for the degree of Doctor of Philosophy at Virginia Commonwealth University.
\end{abstract}

Virginia Commonwealth University, 2016

Major Director: David C. Wheeler, Assistant Professor, Department of Biostatistics

Cancer incidence is associated with exposures to multiple environmental chemicals, and geographic variation in cancer rates suggests the importance of accommodating spatially varying effects in the analysis of environmental chemical mixtures and disease risk. Traditional regression methods are challenged by the complex correlation patterns inherent among cooccurring chemicals, and the applicability of geographically weighted regression models is limited in the setting of environmental chemical risk analysis. In comparison to traditional methods, weighted quantile sum (WQS) regression performs well in the identification of important environmental exposures, but is limited by the assumption that effects are fixed over space. We present an extension of the WQS method that models spatially varying chemical mixture effects called local weighted quantile sum (LWQS) regression, and assess through a simulation study its ability to identify important environmental risk factors over space. We use two different approaches to estimate the LWQS model based on variable subspaces. One uses an 
ensemble of variable subsets of the same size, and the other selects the best subset over a range of candidate subset sizes according to the model goodness-of-fit. We assess the performance of both estimation methods in simulated scenarios that incorporate increasingly complex levels of spatial dependency in the model, and consider correlation patterns from observed exposure data. The results demonstrate that LWQS has the ability to replicate spatially dependent mixture effects and can correctly identify important exposures in a mixture of environmental chemicals. In all scenarios, the best subset approach correctly chose an index containing only the important chemicals and improved on the accuracy of the chemical importance weights in comparison with the ensemble solutions. Future work will evaluate if the ensemble subset approach has better relative performance with larger chemical mixtures of highly correlated components. 


\title{
Chapter 1
}

\author{
Introduction and Prospectus
}

\subsection{Introduction}

The connection between environmental exposures and disease risk is a complex multidimensional problem and known public health concern. Humans experience multiple environmental exposures simultaneously, with exposure profiles that typically vary across location and behavior patterns. For example, risk of non-Hodgkin lymphoma (NHL) is suspected to be associated with several chemicals through environmental and occupational routes of exposure (Hartge et al. 2006). Geographic variation in NHL rates further suggest the importance of accounting for spatial variation in environmental factors.

Several methodological challenges arise when modeling the relationship between multiple environmental exposures and disease risk. Exposure patterns are typically complex, with inherently high correlations among co-occurring environmental chemicals (Czarnota et al., 2014). In the presence of such high correlations, traditional regression methods suffer from the effects of collinearity. While shrinkage methods have been developed to address collinearity and high-dimensionality, they have been shown to arbitrarily choose one variable from a group of highly correlated predictors (Zou et al., 2005). Most problematically, this may lead to the incorrect identification of harmful exposures as innocuous in a risk assessment setting. 
Additionally, the relationship between environmental exposures and adverse health outcomes is not always constant across a study area (Czarnota et al., 2014). Current methods for evaluating spatially varying effects in chemical mixtures include geographically weighted regression (GWR) and geographically weighted lasso (GWL). GWR has been shown to be impacted by local collinearity, which can result in coefficient estimates that are correlated locally and across space, have inflated variances, and are at times counterintuitive and contradictory in sign to the global regression estimates (Wheeler, 2007; Tu et al, 2008). In an effort to address the effects of collinearity in GWR, GWL adds a constraint on the magnitude of the estimated regression coefficients (Wheeler, 2009). GWL also performs model selection by potentially shrinking some of the local regression coefficients to zero, thereby diminishing the adverse effects of the correlation pattern. However, this strategy makes it difficult to discern whether a variable is excluded from the model due to lack of association with the outcome or due to correlation with other variables in the model.

In contrast to standard regression methods, weighted quantile sum regression (WQS) has been shown to perform well in the setting of correlated environmental chemicals, and can effectively distinguish between signal and noise in detecting important exposures (i.e., those related to the outcome). The WQS method estimates an overall mixture effect through a weighted linear index of exposures in which the weights are empirically determined. WQS improves the effects of illconditioning from correlated variables by focusing inference in a single direction and constraining the weights to sum to one. While the WQS method provides several advantages in the analysis of environmental exposures, the estimated weights and mixture effect are treated as constant over space. 
Appropriate accommodation of spatially varying effects in the analysis of chemical mixtures may identify previously undetected environmental sources of disease risk. In this work, we develop local weighted quantile sum (LWQS) regression, a spatial extension of the WQS method that accommodates spatially varying model effects. We include a variable subspace approach as a method of diversity generation and potential variable selection strategy. Two different methods are used to estimate the final LWQS parameters - both based on variable subspaces. One uses an ensemble strategy to create local composite estimates across variable subsets of the same size, and the other selects the best subset over a range of candidate subset sizes according to model goodness-of-fit. We assess the performance of LWQS in a simulation study that considers a variety of environmentally relevant scenarios with increasing complexity in the spatial dependency of parameters. Evaluation is focused on accurate variable selection and signal detection over space. The methodology developed herein has the potential to provide valuable insight into the relationship between adverse health effects and complex environmental exposure profiles.

\subsection{Prospectus}

Chapters 2, 3, and 4 are presented as stand-alone manuscripts. Chapter $\mathbf{2}$ details the application of WQS regression in modeling the association between a mixture of 27 chemicals measured in house dust and risk of NHL in four National Cancer Institute - Surveillance, Epidemiology, and End Results (NCI-SEER) centers. The analysis takes a site-specific approach in a preliminary effort to assess the effects of spatially varying exposure patterns among chemical mixtures on the risk of NHL (Czarnota et al., 2014).

In Chapter 3, we assess through simulation studies, the accuracy of WQS regression in detecting subsets of chemicals associated with health outcomes in site-specific analyses and in non-site- 
specific analysis (Czarnota, et al. ${ }^{\mathrm{a}}$, 2015). In order to achieve realistic exposure situations, we base the simulation study on data from the NCI-SEER NHL study in the previous chapter. We also evaluate the performance of several penalized regression methods in comparison to WQS.

In Chapter 4, we evaluate the impact of collinearity on the geographically weighted regression models of GWR and GWL in a chemical exposure and risk assessment context (Czarnota, et al. ${ }^{\text {b }}$ 2015). We assess through a simulation study the ability of GWR and GWL to correctly identify spatially varying chemical effects for a mixture of correlated chemicals within a study area.

Chapter 5 details the methodological development of LWQS. We include a variable subspace strategy and present two approaches to the estimation of the final LWQS parameters. We then conduct a simulation study to assess the performance of LWQS in a variety of environmentally relevant scenarios. The simulated scenarios incorporate differing degrees of spatial dependency and consider correlation structures based on those observed in the NCI-SEER study.

Chapter $\mathbf{6}$ concludes this work with a summary and discussion of our findings. Limitations of the newly developed method are discussed, and directions for future work are presented. 


\section{Chapter 2}

Analysis of Environmental Chemical Mixtures and Non-Hodgkin Lymphoma Risk in the NCISEER NHL Study.

\subsection{Introduction}

Risk of non-Hodgkin lymphoma (NHL) is suspected to be associated with several chemicals through occupational or environmental routes of exposure; geographic variation in NHL rates further suggests the importance of environmental risk factors (Hartge et al. 2006). Positive associations have been found with persistent organochlorine chemicals, including polychlorinated biphenyls (PCBs) (Engel et al. 2007a), particularly PCB congener 180 (Colt et al. 2005; De Roos et al. 2005; Morton et al. 2008), and dichlorodiphenyldichloroethylene (DDE) (Colt et al. 2005; Engel et al. 2007a). An association between NHL overall (Colt et al. 2006) and certain NHL subtypes (Morton et al. 2008) has also been found for residential termite treatment before 1988 (a surrogate for the insecticide chlordane). Several studies have found higher risk of NHL among persons living in areas with industrial emissions to air or industrial waste exposure (Bithell et al. 1994; De Roos et al. 2010; Dreiher et al. 2005; Floret et al. 2003; Franchini et al. 2004; Goldberg et al. 1999; Pronk et al. 2013).

Existing studies of environmental chemical exposures and NHL generally considered only single-chemical risk or total exposure within specific chemical groups, such as PCBs (Colt et al. 2005; De Roos et al. 2005), but did not consider the effects of simultaneous exposure to multiple diverse chemicals or environmental risk factors. Because individuals are exposed to many 
chemicals simultaneously, it is of particular importance to examine the relationship between chemical mixtures and disease risk. Additionally, the analysis of multiple chemical exposures must also consider the inherent correlations among co-occurring environmental chemicals. The complex correlation pattern among chemical exposures and subsequent issue of collinearity has not been directly addressed in studies of NHL or other diseases.

Some studies of environmental factors and disease risk consider many exposures (Everett et al. 2008), sometimes controlling for multiple comparisons in so-called environment-wide association studies (Patel et al. 2010; Patel et al. 2012), but they use separate regression models for each environmental exposure. This type of analysis ignores that environmental exposures may interact (Engel et al. 2007b; Porta et al. 2012). Studies also examine pairwise correlation coefficients between environmental factors (Ioannidis et al. 2009; Patel et al. 2012), but most do not account for the correlation among factors in statistical models. The lack of statistical independence observed among exposures presents challenges to assessing many exposure effects simultaneously in one traditional regression model.

Here, we present an application of the weighted quantile sum (WQS) regression method (Carrico et al. 2014) to estimate an index for 27 correlated environmental chemicals measured in residential carpet dust in a case-control study of NHL. Estimation of chemical weights and the resulting WQS index while considering the correlation between compounds allows us to make generalized inference about the mixture effect and identify the individual chemicals ('bad actors') most strongly associated with NHL. Due to the design of our study in four geographic regions, the analysis took a site-specific approach in a preliminary effort to consider the effects of spatially varying levels of exposures among chemical mixtures. 


\subsection{Methods}

\subsubsection{Study population}

We conducted a population-based case-control study of NHL in four National Cancer InstituteSurveillance Epidemiology and End Results Program (NCI-SEER) study sites

(http://seer.cancer.gov/). The study design has been previously described (Colt et al. 2004;

Wheeler et al. 2011). Briefly, the study was conducted in Iowa, Los Angeles County, and the metropolitan areas of Detroit (Macomb, Oakland, and Wayne counties) and Seattle (King and Snohomish counties). Eligible cases were aged 20-74 years, diagnosed with a first primary NHL between July 1998 and June 2000, and uninfected with HIV. In Seattle and Iowa, all consecutive cases were chosen. In Detroit and Los Angeles, all African American cases and a random sample of white (regardless of Hispanic ethnicity) cases were eligible for study, allowing for oversampling of African American cases. Of the 2,248 potentially eligible cases, 320 (14\%) died before they could be interviewed, 127 (6\%) were not located, 16 (1\%) had moved away, and 57 (3\%) had physician refusals. Of the 1,728 remaining cases, $1,321(76 \%)$ participated. Controls $(\geq$ 65 years) were selected from Center for Medicare and Medicaid Services files

(http://dnav.cms.gov/) or the general population using random digit dialing ( $<65$ years) and were frequency matched to cases by sex, age (within 5-year groups), race, and study site. Of the 2,409 potentially eligible controls, 2,046 were able to be located and contacted, and 1,057 (52\%) of these subjects participated. The study was approved by the human subjects review boards at all participating institutions. Written informed consent was obtained from each participant. Computer-assisted personal interviews were conducted in the home of each participant. Interviewers asked about demographics including race and education, age of the home, housing type, the presence of oriental rugs, pesticide use in the home and garden, residential and occupational histories, and other factors. 


\subsubsection{Dust samples and laboratory analysis}

As described in detail (Colt et al. 2004; Colt et al. 2005), dust was collected between February 1999 and May 2001 from vacuum cleaners of participants who gave permission (93\% of cases, 95\% of controls) and who had used their vacuum cleaner within the past year and owned at least half their carpets or rugs for 5 years or more (695 cases (57\%), 521 controls (52\%). Dust samples from 682 cases (98\%) and 513 controls (98\%) were successfully analyzed between September 1999 and September 2001.

Exposure to a mixture of 27 chemicals measured in house dust (5 PCBs, 7 polycyclic aromatic hydrocarbons (PAHs), and 15 pesticides) was of interest. The PCBs were congeners 105, 138, 153, 170, and 180. The PAHs were benz(a)anthracene, benzo(a)pyrene, benzo(b)fluoranthene, benzo(k)fluoranthene, chrysene, dibenz(ah)anthracene, and indeno(1,2,3-cd)pyrene. The pesticides were $\alpha$-chlordane, $\gamma$-chlordane, carbaryl, chlorpyrifos, cis-permethrin, transpermethrin, 2,4-dichlorophenoxyacetic acid (2,4-D), DDE, dichlorodiphenyltrichloroethane (DDT), diazinon, dicamba, methoxychlor, $o$-phenylphenol, pentachlorophenol, and propoxur. Extraction and analysis were performed on 2-g aliquots of dust samples using gas chromatography/mass spectrometry (GC/MS) in selected ion monitoring mode. Concentrations were quantified using the internal standard method. Usual detection limits were: $20.8 \mathrm{ng} / \mathrm{g}$ of dust for $\alpha$-chlordane, $\gamma$-chlordane, DDE, DDT, propoxur, o-phenylphenol, PAHs, and PCBs; 42 $84 \mathrm{ng} / \mathrm{g}$ for chlorpyrifos, diazinon, cis-permethrin, dicamba, pentachlorophenol, and 2,4-D; and $121-123 \mathrm{ng} / \mathrm{g}$ for carbaryl and trans-permethrin. Changes in analytic procedures during the study resulted in increased detection limits for methoxychlor (from 20.7 to $62.5 \mathrm{ng} / \mathrm{g}$ ). A small proportion of samples weighing less than $2 \mathrm{~g}$ had detection limits that were higher than the usual detection limits. 
The laboratory measurements for the 27 analytes contained various types of "missing data," primarily when the concentration was below the minimum detection level. To a lesser extent, missing data occurred when there was co-elution between the target chemical and interfering compounds. Chemical concentrations were assumed to follow a log-normal distribution, and data were imputed using a "fill-in" approach to create ten complete datasets for each of the 27 analytes. Details about the imputation of analyte values have been published previously (Colt et al. 2004; Lubin et al. 2004).

A total of $N=1180$ subjects with complete dust analysis results and covariate values were included in this analysis. The sample included 508 (43\%) controls and $672(57 \%)$ cases, and was predominantly white $(88 \%)$ with an average age of 60 years (standard deviation $=11.2$ ). Of these 1180 subjects, 202 (17\%) were from the Detroit study site, 340 (29\%) from Iowa, 292 (25\%) from Los Angeles, and 346 (29\%) from Seattle.

\subsubsection{Statistical Analysis}

In previous analyses of individual chemicals in the study population overall, we evaluated NHL risk comparing tertiles or other groupings of levels above the detection limit to those with no detectable level of the chemical (Colt et al. 2005; Colt et al. 2006; Hartge et al. 2005). Study site specific risk estimates were not presented in these publications. Here, we used a weighted quartile sum approach in conjunction with non-linear logistic regression to evaluate the effect of several chemical exposures together on the risk of NHL. Exposure to a mixture of 27 chemicals measured in house dust was evaluated overall and in study site-specific models. All models were adjusted for gender, age at diagnosis (cases)/selection date (controls), race, and level of education. Age was treated as continuous, race was dichotomized as white or non-white, and 
education was treated as ordinal (grouped as $<12,12-15$, and $\geq 16$ years). In the overall model, we also adjusted for study site.

The WQS method (Carrico et al. 2014) is constrained to have associations in the same direction for chemical exposures and risk, and is designed for variable selection over prediction. WQS regression estimates a weighted linear index in which the weights are empirically determined through the use of bootstrap sampling. The approach considers data with $c$ correlated components scored as ordinal variables into quantiles (here, quartiles) that are reasonable to combine (i.e., all chemicals) into an index and potentially have a common adverse outcome. The weights are constrained to sum to 1 and be between 0 and 1, thereby reducing dimensionality and addressing issues associated with collinearity. For this analysis, the $c=27$ chemical concentrations were scored into quartiles based on the case and control data combined and denoted by $q_{i}$, where $q_{i}=0,1,2$, or 3 for $i=1$ to $c$. A total of $B=100$ bootstrap samples (of the same size as the total sample, $N=1180$ ) were generated from the full dataset and used to estimate the unknown weights, w, that maximized the likelihood for $b=1$ to $B$ for the following model

$$
g(\mu)=\beta_{0}+\beta_{1}\left(\sum_{i=1}^{c} w_{i} q_{i}\right)+\left.\mathbf{z}^{\prime} \varphi\right|_{b}
$$

subject to the constraints $\left.\sum_{i=1}^{c} w_{i}\right|_{b}=1$ and $0 \leq w_{i} \leq 1$ for $i=1$ to $c$. In the above equation, $w_{i}$ represents the weight for the $i^{\text {th }}$ chemical component $q_{i}$ and the term $\sum_{i=1}^{c} w_{i} q_{i}$ represents a weighted index for the set of $c$ chemicals of interest. Furthermore, $\mathbf{z}$ denotes a vector of covariates determined prior to estimation of the weights, $\varphi$ are the coefficients for the covariates 
in $\mathbf{z}$, and $g($.) is any monotonic and differentiable link function that relates the mean, $\mu$, to the predictor variables in the right hand side of the equation. Because the outcome variable of interest in this analysis is binary (case status), a logit link was assumed for $g$.

For each bootstrap sample, the $p$-value of $\beta_{1}$, the parameter estimate for the weighted index, was used to evaluate the statistical significance of the estimated vector of weights $(\alpha=0.10)$. The weighted quantile score was then estimated as $W Q S=\sum_{i=1}^{c} \bar{w}_{i} q_{i}$, where $\bar{w}_{i}=\frac{1}{n_{B}} \sum_{j=1}^{n_{B}} w_{i j}$, and $n_{B}$ is the number of bootstrap samples in which $\beta_{1}$ was significant. Finally, the significance of the WQS index was determined using the original data set and the model

$$
g(\mu)=\beta_{0}+\beta_{1} W Q S+\mathbf{z}^{\prime} \boldsymbol{\varphi}
$$

where $\exp \left(\beta_{1}\right)$ is the odds ratio (OR) associated with a unit (quartile) increase in the weighted sum of exposure quartiles (WQS index).

Weights estimated from the full data set were used to create a WQS index denoted as WQS. In addition to $\mathrm{WQS}_{\mathrm{F}}$, four site-specific indices (denoted as $\mathrm{WQS}_{\mathrm{D}}, \mathrm{WQS}_{\mathrm{I}}, \mathrm{WQS}_{\mathrm{L}}$, and $\mathrm{WQS}_{\mathrm{S}}$ ) were estimated using data from each site. Differences in the distributions of the chemical concentrations across sites prohibited the use of quantiles based on the full data set in the estimation of site-specific weights; therefore, we used site-specific quartiles based on the combined case control distribution to estimate site-specific indices. The association between the WQS indices and NHL was examined by testing each index within its respective data set, with statistical significance set at $\alpha=0.05$. The primary statistical analysis was done with one randomly selected imputation dataset. A secondary analysis estimated WQS indices for all ten imputed datasets to assess sensitivity of the results to the data imputation. 
We conducted further analyses of major subtypes of NHL: diffuse large B-cell (DLBCL), follicular, small lymphocytic/chronic lymphocytic leukemia/ (SLL/CLL), marginal zone, other lymphomas, and lymphomas where subtype was not specified/unknown (not otherwise specified [NOS]). Our study primarily included SLL rather than CLL (Morton et al. 2008). Other lymphomas consisted of mantle cell lymphoma, lymphoplasmacytic lymphoma, Burkitt lymphoma/leukemia, mycosis fungoides/Sézary syndrome, and peripheral T-cell lymphoma. We fitted WQS regression models separately for each of these groups to determine if the mixture effect varied by subtype using all 508 controls in each model.

As a comparison to the WQS regression results, we also conducted single chemical analyses (one-by-one) for all of the data (adjusted for study site) and separately within each study site using study-site specific cutpoints based on the distributions among cases and controls combined. Models were adjusted for gender, age, race, and level of education. Odds ratios comparing each of the three highest quartiles to the first quartile of exposure were estimated for each individual chemical. Given the exploratory nature of these analyses, no adjustments were made for multiple comparisons.

\subsection{Results}

Characteristics of the study population are summarized overall and by study site in Table 2.1. The demographics were similar across the four sites, with the exceptions of race (varying from $73 \%$ white in Los Angeles to $99 \%$ white in Iowa) and education (individuals with $\geq 16$ years of education ranged from $19 \%$ in Iowa to $36 \%$ in Seattle). 
Table 2.1: Characteristics of the NCI-SEER NHL study population overall and by study site.

\begin{tabular}{lccccc} 
& $\begin{array}{c}\text { All Sites } \\
(\mathrm{N}=1180)\end{array}$ & $\begin{array}{c}\text { Detroit } \\
(\mathrm{N}=202)\end{array}$ & $\begin{array}{c}\text { Iowa } \\
(\mathrm{N}=340)\end{array}$ & $\begin{array}{c}\text { Los Angeles } \\
(\mathrm{N}=292)\end{array}$ & $\begin{array}{c}\text { Seattle } \\
(\mathrm{N}=346)\end{array}$ \\
\cline { 2 - 6 } Characteristic & $\mathrm{n}(\%)$ & $\mathrm{n}(\%)$ & $\mathrm{n}(\%)$ & $\mathrm{n}(\%)$ & $\mathrm{n}(\%)$ \\
\hline Case Status & & & & & \\
$\quad$ Control & $508(43)$ & $75(37)$ & $147(43)$ & $125(43)$ & $161(47)$ \\
$\quad$ Case & $672(57)$ & $127(63)$ & $193(57)$ & $167(57)$ & $185(53)$ \\
Age (1) years) & $60 \pm 11.2$ & $58 \pm 11.3$ & $61 \pm 11.4$ & $60 \pm 11.2$ & $59 \pm 10.8$ \\
Sex & & & & & \\
$\quad$ Male & $631(54)$ & $114(56)$ & $181(53)$ & $163(56)$ & $173(50)$ \\
$\quad$ Female & $549(47)$ & $88(44)$ & $159(47)$ & $129(44)$ & $173(50)$ \\
Race & & & & & \\
$\quad$ White & $1033(88)$ & $164(81)$ & $336(99)$ & $213(73)$ & $320(92)$ \\
$\quad$ Non-white & $147(12)$ & $38(19)$ & $4(1)$ & $79(27)$ & $26(8)$ \\
Education & & & & & \\
$\quad<12$ years & $106(9)$ & $24(12)$ & $33(10)$ & $30(10)$ & $19(5)$ \\
$\quad$ 12-15 years & $741(63)$ & $123(61)$ & $244(72)$ & $171(59)$ & $203(59)$ \\
$\geq 16$ years & $333(28)$ & $55(27)$ & $63(19)$ & $91(31)$ & $124(36)$ \\
\hline
\end{tabular}

${ }^{\mathrm{a}}$ Continuous variable summarized using mean \pm standard deviation.

The distribution of the pairwise Spearman correlations of the chemical concentrations was complex (see Appendix A, Figure A1), with pairwise correlations ranging from slightly negative $(\mathrm{r}=-0.15)$ to nearly perfect correlation $(\mathrm{r}=0.99$ for cis- and trans-permethrin). Of the 351 unique pairwise correlations, 289 were significant $(p<0.05)$. Correlation among the PAHs ranged from 0.87 to 0.96 (all significant), and correlation among the PCBs ranged from 0.69 to 0.91 (all significant). The correlation among pesticides was generally weaker ( $84 \%$ significant) with an interquartile range of 0.06 to 0.26 . Median concentrations of PCBs were generally similar across the four sites, although the concentration distributions were more positively skewed in Detroit and Seattle than in Iowa and Los Angeles (see Appendix A, Figure A2). Chemical concentrations for the PAHs and pesticides varied considerably by site. Concentrations for all seven PAHs were elevated (higher than in other locations) in Detroit, while pesticide 
concentrations were elevated in Iowa (e.g., carbaryl, 2,4-D, methoxychlor, and dicamba) and Los Angeles (e.g., $\gamma$-chlordane, trans-permethrin, diazinon, and propoxur) (data not shown).

The WQS index for the overall study population was significantly associated with NHL ( $p=$ 0.006, Table 2.2). More specifically, a quartile increase in the WQS index resulted in an increase of $1.30(95 \%$ CI: $1.08,1.56)$ in the odds of NHL in the overall study population. In the sitespecific analyses, odds ratios for a quartile increase in the Detroit $(\mathrm{OR}=1.71 ; 95 \% \mathrm{CI}=1.02$, 2.92), Iowa $(\mathrm{OR}=1.76 ; 95 \% \mathrm{CI}=1.23,2.53)$ and Los Angeles $(\mathrm{OR}=1.44 ; 95 \% \mathrm{CI}=1.00$, 2.08) indices were significantly associated with NHL. The ORs for each of the five WQS indices were generally robust to the analyte imputation (see Appendix A, Figure A3). Over the ten imputations, the average OR for a quartile increase in the WQS index for the study population overall was 1.25 , while the average site-specific ORs were 1.38 for Detroit, 1.67 for Iowa, 1.61 for Seattle, and 1.45 for Los Angeles. ORs were significant for only one imputation in Detroit, and four of the ten imputations in Seattle.

Table 2.2: Associations between non-Hodgkin lymphoma and the weighted quantile sum regression index in the study population and in each study site.

\begin{tabular}{llll} 
Parameter & $\mathrm{N}$ & Odds Ratio $^{\mathrm{a}}(95 \% \mathrm{CI})$ & $p$-value \\
\hline $\mathrm{WQS}_{\mathrm{F}}$ & 1180 & $1.30(1.08,1.56)$ & 0.006 \\
WQS $_{\mathrm{D}}$ & 202 & $1.71(1.02,2.92)$ & 0.045 \\
WQS $_{\mathrm{I}}$ & 340 & $1.76(1.23,2.53)$ & 0.002 \\
WQS $_{\mathrm{L}}$ & 292 & $1.44(1.00,2.08)$ & 0.049 \\
WQS $_{\mathrm{S}}$ & 346 & $1.39(0.97,1.99)$ & 0.071 \\
\hline
\end{tabular}

Abbreviations: F, full data set; D, Detroit; I, Iowa; L, Los Angeles; S, Seattle.

${ }^{a}$ Estimated odds ratios are associated with a unit increase in the WQS index. All models are adjusted for gender, race, education, and age. The model for the study population (i.e., the full data set) was also adjusted for study site.

The estimated chemical weights for each WQS index are shown in Table 2.3. Note that each weight would be 0.037 if all chemicals in the index received equal weight. The most heavily 
weighted chemicals in the index for the overall data set were PCB 180 (weight $w=0.32$ ),

propoxur $(w=0.17), \operatorname{DDE}(w=0.08), \gamma$-chlordane $(w=0.08)$, and benzo $(\mathrm{k})$ fluoranthene $(w=$ 0.07). The weight for PCB 180 was more than eight times the weight expected if all chemicals were equal.

Table 2.3: Weighted quantile sum regression index weights ${ }^{\mathrm{a}}$ estimated in the study population and in each study site.

\begin{tabular}{|c|c|c|c|c|c|}
\hline Chemical & $\begin{array}{r}\text { Detroit } \\
(p=0.045)\end{array}$ & $\begin{array}{r}\text { Iowa } \\
(p=0.002)\end{array}$ & $\begin{array}{r}\text { Los Angeles } \\
\quad(p<0.049)\end{array}$ & $\begin{array}{r}\text { Seattle } \\
(p=0.071)\end{array}$ & $\begin{array}{r}\text { All Sites } \\
(p=0.006)\end{array}$ \\
\hline PCB 105 & 0.01 & 0.07 & 0.03 & 0.01 & 0.02 \\
\hline PCB 138 & $<0.005$ & 0.01 & 0.01 & $<0.005$ & $<0.005$ \\
\hline PCB 153 & $<0.005$ & 0.01 & 0.12 & 0.07 & 0.02 \\
\hline PCB 170 & 0.17 & 0.01 & 0.01 & 0.01 & 0.03 \\
\hline PCB 180 & 0.18 & 0.02 & 0.01 & 0.14 & 0.32 \\
\hline benz(a)anthracene & $<0.005$ & $<0.005$ & $<0.005$ & 0.09 & $<0.005$ \\
\hline benzo(b)fluoranthene & $<0.005$ & $<0.005$ & 0.10 & $<0.005$ & $<0.005$ \\
\hline benzo(k)fluoranthene & $<0.005$ & $<0.005$ & 0.30 & 0.01 & 0.07 \\
\hline benzo(a)pyrene & 0.07 & 0.04 & $<0.005$ & 0.03 & 0.06 \\
\hline chrysene & $<0.005$ & $<0.005$ & $<0.005$ & $<0.005$ & $<0.005$ \\
\hline dibenz(ah)anthracene & $<0.005$ & 0.03 & 0.03 & 0.01 & 0.01 \\
\hline indeno $(1,2,3-c d)$ pyrene & 0.03 & $<0.005$ & $<0.005$ & $<0.005$ & $<0.005$ \\
\hline$\alpha$-chlordane & 0.02 & 0.07 & 0.03 & 0.01 & 0.04 \\
\hline$\gamma$-chlordane & $<0.005$ & 0.12 & 0.03 & 0.01 & 0.08 \\
\hline carbaryl & 0.01 & $<0.005$ & 0.03 & 0.01 & 0.01 \\
\hline chlorpyrifos & 0.01 & 0.02 & $<0.005$ & $<0.005$ & $<0.005$ \\
\hline cis-permethrin & 0.09 & 0.01 & 0.03 & 0.02 & $<0.005$ \\
\hline trans-permethrin & 0.06 & $<0.005$ & 0.01 & 0.09 & 0.03 \\
\hline $2,4-\mathrm{D}$ & 0.05 & $<0.005$ & 0.11 & $<0.005$ & $<0.005$ \\
\hline DDE & $<0.005$ & 0.11 & 0.07 & 0.14 & 0.08 \\
\hline DDT & 0.01 & $<0.005$ & 0.01 & 0.01 & $<0.005$ \\
\hline diazinon & 0.01 & $<0.005$ & $<0.005$ & 0.02 & $<0.005$ \\
\hline dicamba & 0.09 & $<0.005$ & 0.06 & $<0.005$ & $<0.005$ \\
\hline methoxychlor & 0.12 & 0.01 & $<0.005$ & $<0.005$ & $<0.005$ \\
\hline$o$-phenylphenol & $<0.005$ & 0.11 & $<0.005$ & 0.06 & 0.04 \\
\hline pentachlorophenol & $<0.005$ & 0.06 & $<0.005$ & 0.09 & 0.01 \\
\hline propoxur & 0.05 & 0.30 & 0.01 & 0.16 & 0.17 \\
\hline
\end{tabular}

${ }^{a} p$-value associated with the estimated weighted quantile sum regression index parameter given in Table 2.2. 
The chemicals most heavily weighted in the index varied by site (Table 2.3). PCBs were more heavily weighted in the urban study sites of Detroit, Los Angeles, and Seattle, and pesticides were more heavily weighted in Iowa, an agricultural state. PCB 180 was the most heavily weighted chemical in Detroit $(w=0.18)$, followed by PCB $170(w=0.17)$ and the organochlorine pesticide methoxychlor $(w=0.12)$. In Los Angeles, the PAH benzo(k)fluoranthene had the highest weight $(w=0.30)$, followed by PCB $153(w=0.12)$ and the herbicide $2,4-\mathrm{D}(w=0.11)$. In Seattle, propoxur had the highest weight $(w=0.16)$, followed by PCB $180(\mathrm{w}=0.14)$ and $\operatorname{DDE}(w=0.14)$. The pesticides propoxur $(w=0.30), \gamma$-chlordane $(w$ $=0.12), \mathrm{DDE}(w=0.11)$, and $o$-phenylphenol $(w=0.11)$ had the highest weights in Iowa. Chemicals that were highly weighted in more than one site included PCB 180 (Detroit and Seattle), propoxur (Iowa and Seattle), and DDE (Iowa and Seattle).

The distributions of the weights for PCB 180, propoxur, and benzo(k)fluoranthene, three highly weighted chemicals, differed greatly across the sites (see Appendix A, Figure A4). Although PCB 180 was weighted prominently overall, and in Detroit and Seattle, the distribution of its weights was centered near 0 in both Iowa and Los Angeles. Benzo(k)fluoranthene had a distribution of weights with a median above 0 only in Los Angeles. The weights for propoxur were mostly distributed above 0 in Iowa, but had a median of 0 for Los Angeles. The distributions of weights for propoxur were similar in Seattle and the full study population.

Of the 672 cases of NHL, 31\% were classified as DLBCL, 23\% as follicular, $10 \%$ as SLL/CLL, $9 \%$ as marginal zone, $14 \%$ as other, and $13 \%$ as NOS. The distribution of cases across subtypes was similar for each site (data not shown). WQS regression results by subtype are shown in Table 2.4. ORs for a one quartile increase in the index were statistically significant for follicular 
lymphomas $(\mathrm{OR}=1.47 ; 95 \% \mathrm{CI}=1.08,2.00)$, marginal zone lymphomas $(\mathrm{OR}=2.06 ; 95 \% \mathrm{CI}=$ $1.25,3.47)$, and the subtype other $(\mathrm{OR}=2.26 ; 95 \% \mathrm{CI}=1.55,3.34)$.

Table 2.4: Associations between non-Hodgkin lymphoma subtypes and the weighted quantile sum regression index in the study population.

\begin{tabular}{lrrr} 
Parameter & $\mathrm{N}_{\text {cases }}$ & Odds Ratio $^{\mathrm{a}}(95 \% \mathrm{CI})$ & $p$-value \\
\hline WQS $_{\text {DLBCL }}$ & 207 & $1.26(0.94,1.71)$ & 0.128 \\
WQS $_{\text {Follicular }}$ & 157 & $1.47(1.08,2.00)$ & 0.014 \\
WQS $_{\text {SLLCLL }}$ & 67 & $1.26(0.83,1.93)$ & 0.273 \\
WQS $_{\text {MarginalZone }}$ & 61 & $2.06(1.25,3.47)$ & 0.006 \\
WQS $_{\text {Other }}$ & 91 & $2.26(1.55,3.34)$ & $<0.001$ \\
WQS $_{\text {NOS }}$ & 89 & $1.32(0.91,1.92)$ & 0.144 \\
\hline
\end{tabular}

Abbreviations: $\mathrm{N}_{\text {cases }}$, number of cases; WQS, weighted quantile sum index; DLBCL, diffuse large B-cell; SLL/CLL chronic lymphocytic leukemia/small lymphocytic; NOS, not otherwise specified.

${ }^{a}$ Estimated odds ratios are associated with a unit increase in the WQS index. Models are adjusted for gender, race, education, age, and study site.

For the individual chemical analyses, the OR for the fourth vs. first quartile of exposure for each chemical is listed in Table A1 (Appendix A) for the overall analyses and in Table A2 (Appendix A) for the site-specific analyses. For the overall study population, PCB 180 was significantly associated with NHL (OR $=1.55 ; 95 \%$ CI: 1.11, 2.17 for the fourth vs. first quartile). Associations between NHL and the remaining PCBs were positive (ORs $\geq 1.20$ ) but not significant (p-values $\leq 0.29)$. Additionally, although non-significant, the ORs for each of the PAHs were less than one. With respect to the pesticides, the highest quartiles of $\alpha$-chlordane (OR $=1.40 ; 95 \%$ CI: $0.99,1.98)$ and $\gamma$-chlordane $(\mathrm{OR}=1.35 ; 95 \% \mathrm{CI}: 0.95,1.92)$ were positively associated with NHL, while fourth quartile levels of chlorpyrifos (OR $=0.73 ; 95 \% \mathrm{CI}$ : 0.52 , 1.02), 2,4-D (OR $=0.70 ; 95 \%$ CI: 0.48, 1.03), and dicamba $(\mathrm{OR}=0.74 ; 95 \%$ CI: $0.53,1.04)$ were inversely associated with NHL. 
In the site-specific analyses of individual chemicals (highest vs. lowest quartiles), PCB 180 was significantly associated with NHL in Detroit (OR $=2.87 ; 95 \%$ CI: $1.19,6.91)$. In Iowa, associations were positive and significant for $\alpha$-chlordane (OR $=2.18$; 95\% CI: $1.15,4.14), \gamma$ chlordane $(\mathrm{OR}=2.25 ; 95 \% \mathrm{CI}: 1.20,4.24), \mathrm{DDE}(\mathrm{OR}=1.96 ; 95 \% \mathrm{CI}: 1.05,3.68)$, and propoxur $(\mathrm{OR}=2.02 ; 95 \% \mathrm{CI}: 1.09,3.78)$; whereas associations were negative and significant for 2,4-D $(\mathrm{OR}=0.36 ; 95 \% \mathrm{CI}: 0.19,0.68)$ and dicamba $(\mathrm{OR}=0.48 ; 95 \% \mathrm{CI}: 0.26,0.90)$. In Los Angeles, benzo(k)fluoranthene was significantly associated with NHL (OR = 2.05; 95\% CI: 1.04, 4.04). In Seattle, no significant positive associations were found, however propoxur $(\mathrm{OR}=1.53 ; 95 \% \mathrm{CI}$ : 0.82, 2.85), DDE (OR = 1.53; 95\% CI: 0.83, 2.84), and PCB 180 (OR = 1.53; 95\% CI: 0.82, 2.85) were nominally associated with NHL. Finally, 2,4-D (OR $=0.53 ; 95 \%$ CI: $0.29,0.97)$ and dicamba $(\mathrm{OR}=0.41 ; 95 \% \mathrm{CI}: 0.22,0.76)$ were both significantly inversely associated with NHL in Seattle.

\subsection{Discussion}

We used weighted quantile sum regression to model the association of a mix of 27 correlated environmental chemicals measured in house dust and risk of NHL in a case-control study in four study centers. We fitted site-specific WQS models and an overall WQS model. We found evidence of an increased risk of NHL associated with an increase in the quantile of the weighted chemical index in the overall study population and in each of the four study sites. These associations were statistically significant for the study population overall and for three of the four study centers (Iowa, Detroit, and Los Angeles), and marginally significant in Seattle.

The most highly weighted chemicals in the overall WQS index were PCB congener 180, propoxur, $\gamma$-chlordane, and DDE. The chemicals most heavily weighted in the site-specific mixture indexes varied by site. Additional chemicals that were relatively highly weighted in site- 
specific models and associated with an increased risk of NHL included PCB 153 (Los Angeles), PCB 170 (Detroit), 2,4-D (Los Angeles), benzo(k)fluoranthene (Los Angeles), methoxychlor (Detroit), and $o$-phenylphenol (Iowa).

By comparison, in single chemical analyses, only PCB 180 was found to be significantly associated with NHL in the study population overall, although $\gamma$-chlordane was marginally significant. In prior analyses of these data using a slightly different approach, $\gamma$-chlordane and DDE were both associated with significantly increased risks of NHL (Colt et al. 2005; 2006). PCB 180 was the only chemical significantly associated with NHL in analyses of individual chemicals in Detroit. In Iowa, $\alpha$ - and $\gamma$-chlordane, DDE, and propoxur were associated with significantly increased risk of NHL. Benzo(k)fluoranthene was the only chemical significantly associated with NHL risk in Los Angeles. There were no chemicals significantly associated with increased risk in Seattle.

WQS regression highlighted some chemicals that were not significantly associated with NHL in prior analyses or by our single chemical analysis here. These included propoxur in the overall study population, and methoxychlor, $o$-phenylphenol, 2,4-D, PCB 153, and PCB 170 in individual study sites. These chemicals had weights that were several times greater than the value associated with equal weight for all chemicals. In addition, chemicals with non-negligible weights factor positively into the weighted quantile sum index and hence were part of the exposure term found to be significantly associated with NHL in the study population and in three of the four study sites.

Generally, the results from our individual chemical analyses supported the WQS regression findings. The chemicals associated with a significant increase in risk of NHL by individual 
chemical analyses were also selected as potential risk factors by WQS regression (received nonnegligible weights). These include PCB 180 (Detroit), propoxur (Iowa), benzo(k)fluoranthene (Los Angeles), and $\gamma$-chlordane (Iowa). However, WQS was able to place non-negligible weights on several additional chemicals that were not significantly associated with increased risk in individual chemical analyses. When considered individually, many of these chemicals had elevated ORs that may be potentially meaningful, but were not found to be significantly associated with NHL, likely due to a lack of power. For example, single chemical analysis for propoxur in the full study population resulted in an $\mathrm{OR}$ of $1.27(95 \% \mathrm{CI}=0.90,1.79 ; \mathrm{p}$-value $=$ 0.18) overall. WQS regression selected this chemical as a strong risk factor, giving it a substantial weight (0.17). In Seattle, the single chemical ORs for DDE $(\mathrm{OR}=1.53 ; 95 \% \mathrm{CI}=$ $0.83,2.84)$ and propoxur $(\mathrm{OR}=1.53 ; 95 \% \mathrm{CI}=0.82,2.85)$ were not significant, but these chemicals received WQS weights of 0.14 and 0.16 , respectively. Additionally, WQS placed zero or negligible index weights on chemicals that were identified as having inverse associations with NHL in the one-by-one analyses, (e.g., diazinon and dicamba).

Our findings also show that chemicals identified as important based on a site-specific WQS index may not be identified as important in an index derived from the full dataset. Similarly, chemicals identified as important in the index developed from the full dataset may not be identified as important in all site-specific indices. These differences are due in part to different concentration ranges across sites and overall (see Appendix A, Figure A2). The differences may also be due to different sources of these chemicals across study sites or differences in correlations with unmeasured exposures or other factors. For example, PAHs in Los Angeles may be correlated with benzene exposure from traffic and sources in Detroit may be different. 
The results illustrate the importance of estimating site-specific weights when developing the chemical mixture index when multiple sites are simultaneously under study.

The main strengths of this analysis are the evaluation of a broad range of environmental chemicals together in one modeling approach, the estimation of the association of a mixture of chemicals with NHL risk, and the estimation of mixture effects by study site. Previous studies have focused on effects of individual chemical exposures. We have used a statistical method that has been shown to have high specificity and adequate sensitivity in identifying important chemicals in regression models in simulation studies (Carrico et al. 2014). In simulation studies based on pairwise correlations of 11 phthalates in NHANES (2005-2008), WQS regression had greater accuracy in identifying the 7 of 11 truly important chemicals (i.e., chemicals set to be related to the outcome) correctly as the correlation of the exposures and the outcome increased from that observed (range of 0.03 to 0.08 ) to 3 times that observed (Carrico et al. 2014). It also showed an improvement in specificity over traditional ordinary regression and popular shrinkage methods (lasso, adaptive lasso and elastic net). WQS regression tends to place negligible weight on components with no correlation with the outcome.

Single chemical analyses are subject to confounding because of the high degree of correlation among chemical exposure concentrations, but WQS regression allows one to consider exposure to several chemicals simultaneously while accounting for collinearity. Estimation of individual chemical weights enables the identification of potentially harmful chemicals while accommodating the complex correlation observed among exposures. Additionally, WQS regression has the advantage of the estimation of a mixture effect and its association with NHL. Because all potentially harmful chemicals (i.e., those receiving non-zero weights) contribute to the estimation of the WQS index, the OR corresponding to the index is interpretable as the 
increase in risk associated with a quartile increase in the index of the mixture of chemicals, allowing for further inference and insight regarding the potentially harmful effects of exposure to these environmental chemicals.

A limitation of WQS regression is that it cannot identify associations in different directions for the components of the index. However, chemicals in our analysis with inverse associations with NHL in the single chemical analyses were estimated to have negligible weight in the WQS index that had a positive association with NHL. A limitation of the risk analysis is the potential for exposure misclassification from the use of chemical concentrations in house dust as a measure of past exposures. Levels of chemicals in carpet dust do not indicate the source of the chemicals or when they entered the home, and do not reflect dietary ingestion. However, dust sampling has important advantages over questionnaire- and biologically-based approaches. Levels in dust are unaffected by difficulties or biases in recall of past activities, and by factors that may influence body burdens of chemicals such as age, body mass index, and disease status or treatment (Colt et al. 2005). Further, with the exception of the persistent organochlorine chemicals (e.g., PCBs, chlordane, and DDT), which are no longer in use, biological measures of many chemicals may reflect only recent exposures due to short half-lives in the body. Carpets act as long-term chemical repositories, and hence chemical concentrations in carpet dust may reflect integrated chemical exposure over the time the carpet was in the home, providing potentially more relevant exposure indicators than a biologic measure of recent exposure. Moreover, studies have found positive associations between chemical concentrations in dust samples and biomarkers of exposure including serum and house dust levels of PCBs (Knobeloch et al. 2012; Rudel et al. 2008) and lead (Lanphear et al. 1998), and urinary and house dust levels of chromium (Stern et al. 1998). 
Another potential limitation was the large number of nonparticipants. However, previous analyses of spatial variation in NHL risk based on eligible nonparticipants and participants in this study population did not substantially change results based on study participants only (Wheeler et al. 2011). Additionally, analyses of potential bias in NHL risk associated with census-tract educational level among eligible nonparticipants and participants found a negligible bias between $1 \%$ and $8 \%$ (Shen et al. 2008). Also, dust samples were collected only from study participants who owned most of their carpets for at least five years; therefore, concentrations may not be representative of cases and controls who moved or replaced their carpets within five years of the interview.

\subsection{Conclusions}

We applied weighted quantile sum regression to estimate the association between NHL and an index for 27 correlated environmental chemicals measured in residential carpet dust. The WQS method allowed us to make generalized inference about the chemical mixture effect and identify the individual chemicals most strongly associated with NHL while considering the correlation between compounds. Using WQS regression, we found a positive association between the chemical index and NHL in the overall study population and in each of the four study sites. The WQS analysis also implicated several chemicals as NHL risk factors that were not associated with NHL when evaluated individually. Our results demonstrate the importance of evaluating chemical mixtures when studying cancer risk. 


\section{Chapter 3}

Assessment of Weighted Quantile Sum Regression for Modeling Chemical Mixtures and Cancer Risk

\subsection{Introduction}

The connection between environmental chemical exposures and human health/diseases (e.g., cancer) is a complex multi-dimensional problem that is of great interest to public health researchers. Further, exposure to environmental chemical mixtures varies across location and behavior patterns. For example, exposure patterns to polycyclic aromatic hydrocarbons (PAHs) from automobile exhaust in dense urban sites such as Detroit differ from those in rural, agricultural sites such as Iowa. However, there are many sources of PAHs besides automobile exhaust, and different sources may explain different levels across space. For example, PAH concentrations have been found to be associated with residence age (Whitehead et al., 2009, Whitehead et al., 2014). Some environmentally persistent chemicals such as polychlorinated biphenyls (PCBs) may be relatively similar in urban and rural areas. Total PCB levels have been found to be positively associated with percentage of developed land or population density and housing age (DellaValle et al., 2013). Regardless, exposure studies demonstrate complex correlation patterns among environmental chemicals that may vary across regions (Czarnota et al., 2014).

Because individuals are exposed to multiple chemicals simultaneously, it is important to examine the relationship between chemical mixtures and disease risk. Our research focus is on developing 
analysis strategies that incorporate larger sets of chemicals, which are more representative to actual human exposure. In such a higher-dimensional framework, some factors may increase risk, some may diminish risk, and others may have no effect on risk. The goal is to use an analysis strategy that most efficiently detects the signals and deemphasizes the noise in the data. Furthermore, we aim to develop and assess methods that accommodate site-specific exposure patterns and have the ability to accurately discern whether or not a chemical is associated with the outcome of interest.

Through weighted quantile sum (WQS) regression (Carrico et al., 2014) we are able to estimate a body burden index within a set of correlated environmental chemicals, and further estimate the association between the index and an outcome of interest. Additionally, the estimated chemical weights allow us to make generalized inference concerning relative chemical importance. WQS regression is constrained to model associations between the outcome and chemicals that are in one direction (all non-negative or all non-positive), making it appropriate in a risk setting where the goal is to identify exposures that are positively associated with a health outcome. As such, WQS regression is designed for variable selection with less emphasis on risk prediction.

Few existing studies of chemical exposure and disease risk have made efforts to consider the impact of spatially varying exposure patterns on the effect of a chemical mixture. One exception is Czarnota et al. (2014), where the authors take a site-specific approach in a preliminary effort to assess the effects of spatially varying exposure patterns among chemical mixtures on the risk of non-Hodgkin lymphoma (NHL). The objective in that work was to apply statistical methods to detect bad actors in environmental chemical mixtures while considering different exposure patterns based on geographic site. The focus here is on assessing through simulation studies the 
accuracy of WQS regression in detecting subsets of chemicals associated with health outcomes (binary and continuous) in site-specific analyses and in non-site-specific analysis. We based the simulation study on data from the National Cancer Institute Surveillance Epidemiology and End Results Program (NCI-SEER) case-control study of NHL to achieve realistic exposure situations while setting which chemicals were truly associated with the health outcomes. For comparison, we also evaluated the performance of several penalized regression methods in correctly classifying chemicals as bad actors or unrelated to the outcome.

\subsection{Methods}

\subsubsection{NCI-SEER Study Population}

The NCI-SEER NHL population-based case-control study design has been previously described (Colt et al., 2004, Wheeler et al., 2011). Briefly, the study was conducted in Iowa, Los Angeles County, and the metropolitan areas of Detroit (Macomb, Oakland, and Wayne counties) and Seattle (King and Snohomish counties). Eligible cases were aged 20-74 years, diagnosed with a first primary NHL between July 1998 and June 2000, and uninfected with HIV. In Seattle and Iowa, all consecutive cases were chosen. In Detroit and Los Angeles, all African American cases and a random sample of white cases were eligible for study, allowing for oversampling of African American cases. Of the 2,248 potentially eligible cases, 320 (14\%) died before they could be interviewed, 127 (6\%) were not located, $16(1 \%)$ had moved away, and $57(3 \%)$ had physician refusals. Of the 1,728 remaining cases, 1,321 (76\%) participated. Controls were selected from Center for Medicare and Medicaid Services files ( $\geq 65$ years) or the general population using random digit dialing ( $<65$ years) and were frequency matched to cases by sex, age, race, and study site. Of the 2,409 potentially eligible controls, 2,046 were able to be located and contacted, and 1,057 (52\%) of these subjects participated. 
Computer-assisted personal interviews were conducted in the home of each participant. Interviewers asked about demographics including race and education, age of the home, housing type, the presence of oriental rugs, pesticide use in the home and garden, residential and occupational histories, and other factors. As described in detail (Colt et al., 2004, Colt et al., 2005), dust was collected between February 1999 and May 2001 from vacuum cleaners of participants who gave permission (93\% of cases, $95 \%$ of controls) and who had used their vacuum cleaner within the past year and owned at least half their carpets or rugs for 5 years or more (695 cases (57\%), 521 controls (52\%)). Dust samples from 682 cases (98\%) and 513 controls (98\%) were successfully analyzed between September 1999 and September 2001.

A total of 27 chemicals were measured in house dust (5 PCBs, 7 PAHs, and 15 pesticides). The PCBs were congeners 105, 138, 153, 170, and 180. The PAHs were benz(a)anthracene, benzo(a)pyrene, benzo(b)fluoranthene, benzo(k)fluoranthene, chrysene, dibenz(ah)anthracene, and indeno(1,2,3-cd)pyrene. The pesticides were $\alpha$-chlordane, $\gamma$-chlordane, carbaryl, chlorpyrifos, cis-permethrin, trans-permethrin, 2,4-dichlorophenoxyacetic acid (2,4-D), DDE, dichlorodiphenyltrichloroethane (DDT), diazinon, dicamba, methoxychlor, o-phenylphenol, pentachlorophenol, and propoxur. Extraction and analysis were performed on 2-g aliquots of dust samples using gas chromatography/mass spectrometry (GC/MS) in selected ion monitoring mode. Concentrations were quantified using the internal standard method. Usual detection limits were: $20.8 \mathrm{ng} / \mathrm{g}$ of dust for $\alpha$-chlordane, $\gamma$-chlordane, DDE, DDT, propoxur, o-phenylphenol, PAHs, and PCBs; $42-84$ ng/g for chlorpyrifos, diazinon, cis-permethrin, dicamba, pentachlorophenol, and 2,4-D; and 121-123 ng/g for carbaryl and trans-permethrin. Changes in analytic procedures during the study resulted in increased detection limits for methoxychlor 
(from 20.7 to $62.5 \mathrm{ng} / \mathrm{g}$ ). A small proportion of samples weighing less than $2 \mathrm{~g}$ had detection limits that were higher than the usual detection limits.

The laboratory measurements for the 27 analytes contained various types of "missing data," primarily when the concentration was below the minimum detection level. To a lesser extent, missing data occurred when there was co-elution between the target chemical and interfering compounds. Chemical concentrations were assumed to follow a log-normal distribution, and data were imputed using a "fill-in" approach to create ten complete datasets for each of the 27 analytes. Details about the imputation of analyte values have been published previously (Colt et al., 2004, Lubin et al., 2004). A total of 1180 participants had available measurements of all 27 chemicals. Of these participants, 508 (43\%) were controls and 672 (57\%) were cases. With respect to study site, 202 (17\%) were from Detroit, 340 (29\%) from Iowa, 292 (25\%) from Los Angeles, and 346 (29\%) from Seattle.

Our primary interest in the NCI-SEER NHL study is the chemical exposure patterns and the correlation structure of the exposures. As illustrated in Figure 3.1, the concentrations among the PCBs were similar across the four sites, while the concentrations of PAHs and pesticides varied considerably by site. More specifically, concentrations for all seven PAHs were notably elevated in Detroit, while elevated concentrations of pesticides were seen in Iowa (ex. 2,4-D and methoxychlor) and Los Angeles (ex. $\alpha$ - and $\gamma$-chlordane and propoxur). 

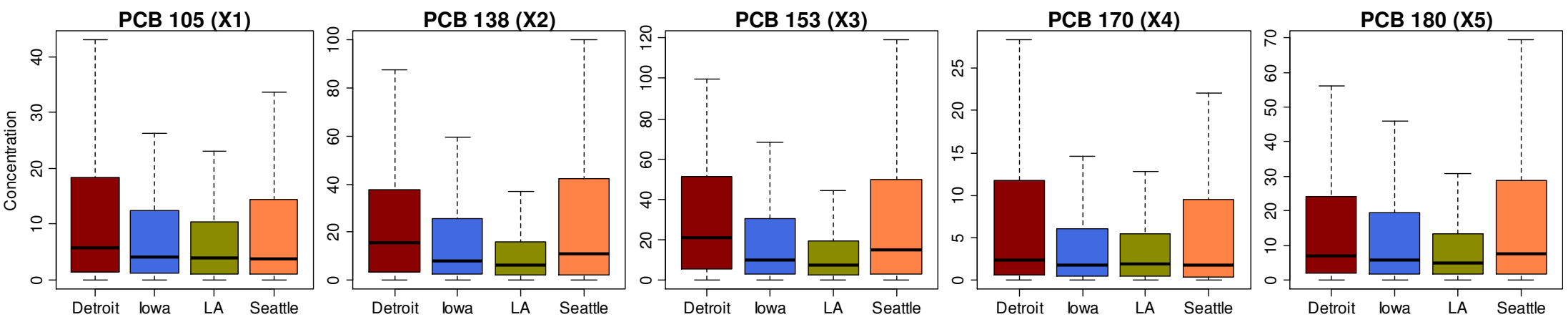

a) PCBs
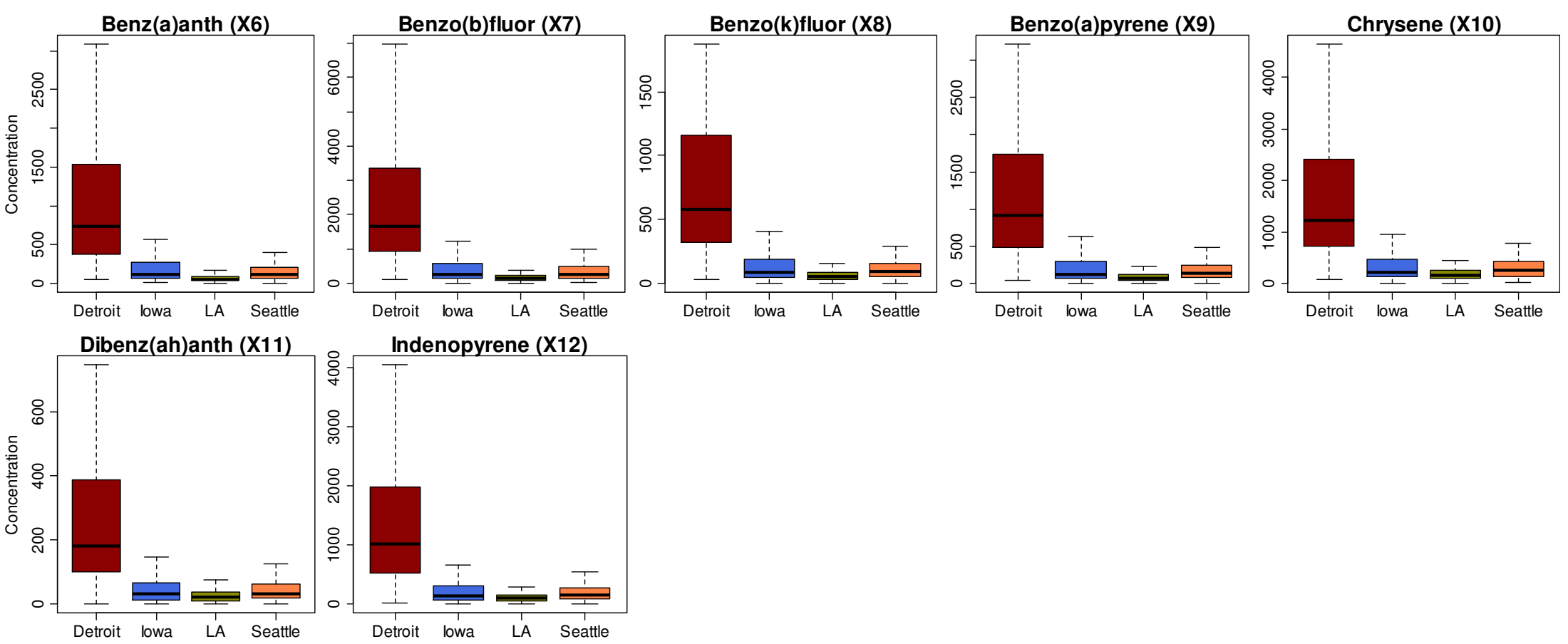

b) PAHs 

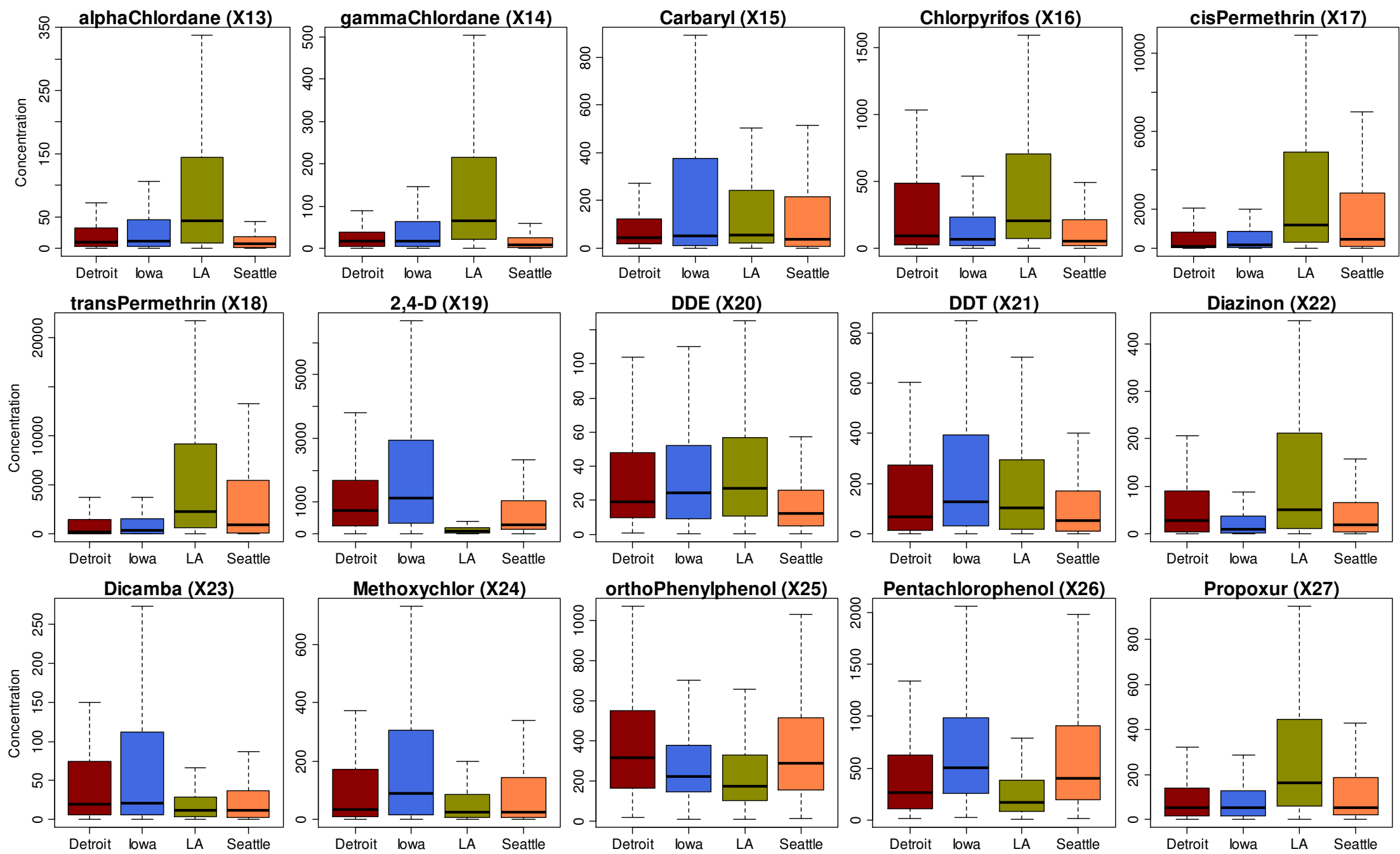

c) Pesticides/Insecticides

Figure 3.1: Distribution of chemical concentrations in the NCI-SEER NHL study for a) PCBs, b) PAHs, and c) Pesticides/Insecticides. 
The site-specific distributions of the Pearson pairwise correlation coefficients among the log transformed concentrations are shown in Figure 3.2. The observed pairwise correlation patterns are complex, with correlations ranging from slightly negative to nearly perfectly correlated within all four sites. When examining the correlations by chemical group, we see that for each site, the PAHs and PCBs demonstrated a high degree of intragroup correlation. The pesticides generally exhibited lesser intragroup correlation, with the exception of the pairwise correlations between metabolites or analogues. For each site, correlation within chemical group is further illustrated in Figure 3.3, and summarized in Table 3.1. We see that the PCBs were most highly correlated in Los Angeles, with $75 \%$ of the intragroup correlations greater than 0.81 . Additionally, we see that the correlation among the PAHs was most pronounced in Detroit (pairwise correlations ranging from 0.95 to 0.99 ) where PAH exposure was highest, and least pronounced in Los Angeles (Interquartile range (IQR) of 0.68 to 0.86 ), were PAH exposure concentration was lowest. As demonstrated by the NHL data, chemical exposure patterns may vary in both concentration and correlation across space, illustrating the need to consider site specific risk analyses in the context of environmental chemical exposure.

Table 3.1: Correlations within chemical group by study site and across the full population in the NCI-SEER NHL study.

\begin{tabular}{|c|c|c|c|}
\hline & PCBs & PAHs & Pesticides/Insecticides \\
\hline & Median (Range) & Median (Range) & Median (Range) \\
\hline Detroit & $0.76(0.68,0.89)$ & $0.98(0.95,0.99)$ & $0.20(-0.09,0.98)$ \\
\hline Iowa & $0.76(0.63,0.93)$ & $0.92(0.80,0.97)$ & $0.17(-0.07,0.98)$ \\
\hline Los Angeles & $0.84(0.80,0.95)$ & $0.80(0.63,0.91)$ & $0.14(-0.06,0.99)$ \\
\hline Seattle & $0.78(0.65,0.95)$ & $0.93(0.81,0.97)$ & $0.16(-0.10,0.97)$ \\
\hline Full & $0.79(0.70,0.94)$ & $0.94(0.86,0.97)$ & $0.14(-0.12,0.98)$ \\
\hline
\end{tabular}



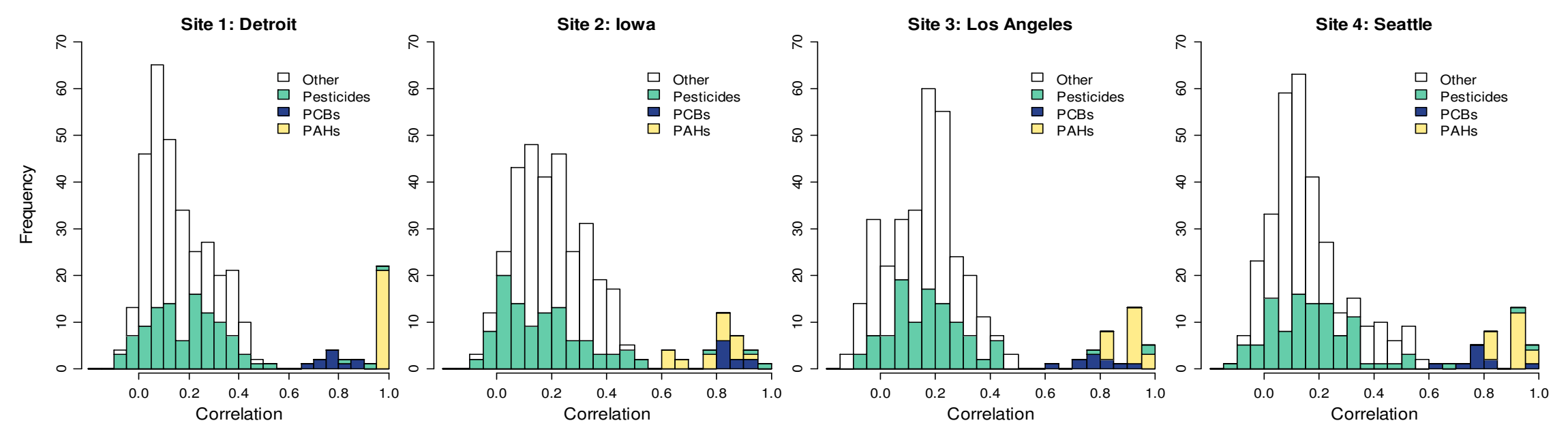

Figure 3.2: Distribution of Pearson pairwise correlation coefficients among chemical concentrations (on the log scale) by study site in the NCI-SEER NHL study
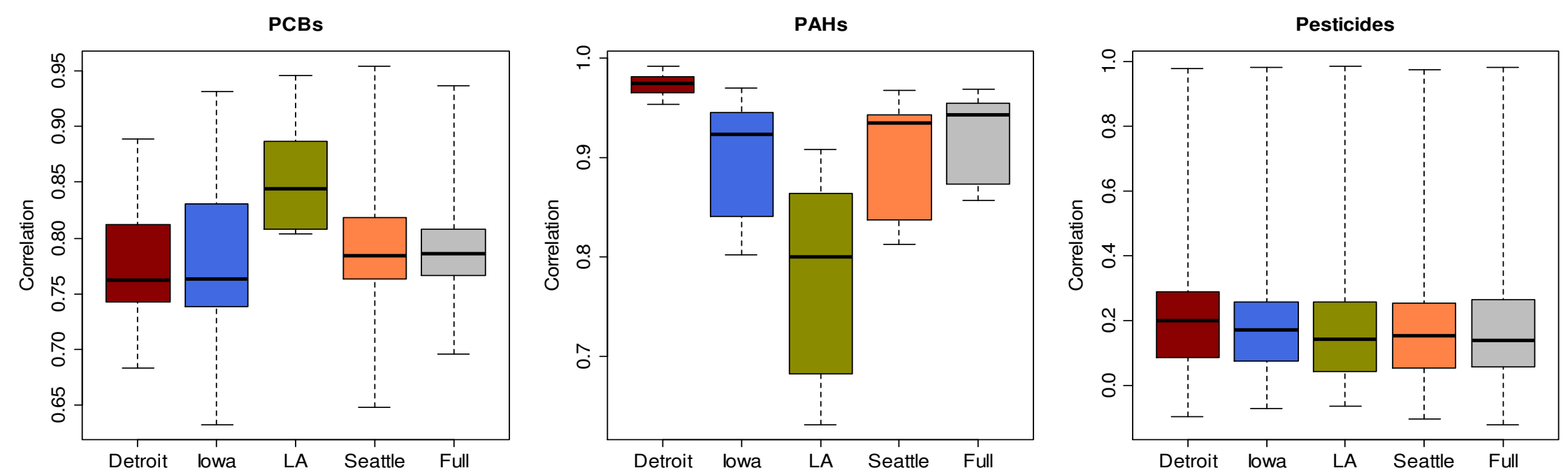

Figure 3.3: Distribution of the observed intergroup correlations for PCBs, PAHs, and pesticides by study site and across the full study population 


\subsubsection{Simulation Study Design}

Using each of the four site-specific correlation structures for the 27 chemicals in the NCI-SEER NHL study, we generated data on a site-specific basis using the observed mean concentrations (from the log scale) and standard deviations, for each of the following three correlation patterns: 1) $65 \%$ of the observed site-specific correlation structure (moderate correlation), 2) $30 \%$ of the observed site-specific correlation structure (mild correlation), and 3) 1\% of the observed sitespecific correlation structure (near independence). We did not include the observed correlation patterns to generate data as the resulting correlation matrices were generally singular and could not be inverted.

The following seven chemicals (one PCB, two PAHs, and four pesticides/insecticides) were set to be associated with the response variable: PCB180 (X5), benzo(k)fluoranthene (X8), benzo(a)pyrene (X9), 2,4-D (X19), DDE (X20), methoxychlor (X24), and propoxur (X27).These chemicals were chosen in an effort to represent a wide range of correlations between and among the bad actors and benign chemicals. Correlation within the seven selected chemicals ranged from -0.08 to 0.94 , with a median correlation of 0.14 . We also ensured that at least one chemical was selected from each chemical group in an attempt to capture the variation in exposure patterns across the study sites. PCB 180 was purposefully selected to represent the PCBs due to its known link to NHL.

Given that chemical exposure patterns differed across site, the association with the outcome variable was assumed true only under the condition that the observed site-specific concentrations were high enough to have a health effect. More specifically, we assumed the association was true within a site if and only if more than $25 \%$ of the site-specific concentrations were higher than the overall median concentration. This condition was satisfied for each of the seven specified 
chemicals in the Detroit, Iowa, and Seattle sites, and thus all seven of the specified chemicals were set to be associated with the response when simulating data. For the Los Angeles site, over $75 \%$ of the observed concentrations for chemicals $\mathrm{X} 8, \mathrm{X} 9$, and $\mathrm{X} 19$ were below the overall median concentration. Therefore, only four chemicals (X5, X20, X24, and X27) were set to be correlated with the outcome in Los Angeles.

For each correlation pattern we simulated data where the selected chemicals (i.e., the prespecified chemicals that satisfied the above condition) had a correlation of 0.1 with the response (weak association with outcome) as well as where the selected chemicals had a correlation of 0.3 with the response (strong association with outcome). To simulate the analyses of case-control study data, we calculated a disease indicator for those subjects with the highest $30 \%$ of the simulated continuous response variable.

We simulated 100 datasets for each set of conditions (correlation pattern and outcome correlation combination), with a sample size of $N=1000$ for each site. The site-specific data sets were split into training and validation sets (50:50 split) and then concatenated to create an overall training and testing data set. Thus, the site specific sample size was $N=1000$ (500 for estimation and 500 for validation), while the overall sample size was $N=4000$ (2000 for estimation and 2000 for validation).

Data were simulated for each site based upon the site-specific observed mean vector (on the log scale) and covariance matrix. The continuous response variable $y$ was assumed to be normally distributed with a mean of 0 and variance of $1, y \sim N(0,1)$, for each site. We used a dampening parameter $k \in(0.65,0.30,0.01)$ to diminish the site-specific correlations to $65 \%, 30 \%$, and $1 \%$ of that observed using the equation $\tilde{\mathbf{R}}_{j}=k\left(\mathbf{R}_{j}-\mathbf{I}\right)+\mathbf{I}$, where $\mathbf{R}_{j}$ denotes the observed matrix 
of correlations for site $j$, and $\tilde{\mathbf{R}}_{j}$ is the corresponding diminished correlation structure. In order to simulate a data set $\mathbf{Y} \sim N(\mathbf{M}, \Sigma)$ with a specified correlation structure for a continuous response variable, $y$, and predictors, $x_{1}, x_{2}, \ldots, x_{c}$, the following method was used according to Carrico et al. (2014):

Let $p=c+1$, and define $\boldsymbol{\rho}_{p \times p}$ as the correlation matrix between and among $y$ and the chemicals in $\mathbf{X}$. Let $\sum_{p \times p}$ denote the corresponding covariance matrix, and define $\mathbf{S}_{p \times 1}$ as the vector of standard deviations. Additionally, let $\mathbf{m}_{p \times 1}$ be the vector of observed sample means for the predictor variables and outcome $y$. We then define the matrix $\mathbf{D}=\operatorname{diag}(\mathbf{S})$, a square matrix of dimension $p \times p$ with diagonal entries consisting of the standard deviations, and impose the desired correlation structure through the relationship between the correlation and variance given by $\Sigma=\mathbf{D} \boldsymbol{\rho D}$.

Next, calculate the Cholesky decomposition, $\mathbf{U}$, of the covariance matrix $\sum$. That is, calculate $\mathbf{U}_{p \times p}$ such that $\sum=\mathbf{U}_{p \times p}^{\prime} \mathbf{U}_{p \times p}$, noting that calculation of the Cholesky decomposition requires that the covariance matrix be positive definite. Simulate $Z_{i} \sim N\left(\mathbf{0}_{p \times 1}, \mathbf{I}_{p}\right)$ for $i=1, \ldots, \mathrm{n}$, and define $\mathbf{Z}^{\prime}=\left[\mathbf{Z}_{1} \cdots \mathbf{Z}_{n}\right]$. In other words, $\mathbf{Z}_{n \times p}=\left[\mathbf{Z}_{1}, \ldots, \mathbf{Z}_{n}\right]$, where each row is p-variate standard normal. Let $\mathbf{M}_{n \times p}=\left[\mathbf{m}_{p \times 1} \mathbf{1}_{1 \times n}\right]^{\prime}$ and define $\mathbf{Y}$ as $\mathbf{Y}_{n \times p}=\mathbf{M}_{n \times p}+\mathbf{Z}_{n \times p} \mathbf{U}_{p \times p}$. Here, $\mathbf{Y}$ is the newly generated data matrix with mean $E(\mathbf{Y})=E(\mathbf{M}+\mathbf{Z U})=\mathbf{M}+\mathrm{E}(\mathbf{Z})=\mathbf{M}$, and variance $\operatorname{Var}(\mathbf{Y})=\operatorname{Var}(\mathbf{M}+\mathbf{Z U})=\operatorname{Var}(\mathbf{M})+\operatorname{Var}(\mathbf{Z U})=\mathbf{U}^{\prime} \operatorname{Var}(\mathbf{Z}) \mathbf{U}=\mathbf{U}^{\prime} \mathbf{U}=\sum$. Thus, $\mathbf{Y}_{n \times p}$ is distributed as $N_{p}(\mathbf{M}, \Sigma)$. 


\subsubsection{Weighted Quantile Sum Regression}

The primary method of risk analysis used in this study was weighted quantile sum regression. The WQS approach estimates a weighted linear index in which the weights are empirically determined through the use of bootstrap sampling. The approach considers data with $c$ components scored into quantiles that are reasonable to combine into an index and potentially have a common outcome. The weights are constrained be between 0 and 1 and sum to 1 , reducing dimensionality and addressing issues that arise with collinearity. For this analysis, the $c$ $=27$ chemical concentrations were scored into quartiles (to reduce the influence of outliers in skewed distributions) denoted by $q_{i}$, where $q_{i} \in\{0,1,2,3\}$ for $i=1$ to $c$. For estimation of the weights, we split the simulated data into training and validation data sets of size $\mathrm{N}_{t}$ and $\mathrm{N}_{\mathrm{v}}$, respectively. A total of $B=100$ bootstrap samples of size $\mathrm{N}_{\mathrm{t}}$ were generated from the training data and used to estimate the unknown weights $w_{i}$ that maximized the likelihood for $b=1$ to $B$ for the following nonlinear model

$$
g(\mu)=\beta_{0}+\beta_{1}\left(\sum_{i=1}^{c} w_{i} q_{i}\right)+\left.\mathbf{z}^{\prime} \varphi\right|_{b}
$$

subject to the constraints $\left.\sum_{i=1}^{c} w_{i}\right|_{b}=1$ and $0 \leq w_{i} \leq 1$ for $i=1$ to $c$. In the above equation, $w_{i}$ represents the weight for the $i^{\text {th }}$ chemical component $q_{i}$, and the term $\sum_{i=1}^{c} w_{i} q_{i}$ represents a weighted index for the set of $c$ chemicals of interest. Furthermore, $\mathbf{z}$ denotes a vector of covariates determined prior to estimation of the weights, and $g$ is a monotonic and differentiable link function that relates the mean, $\mu$, to the predictor variables in the right hand side of the equation. For this analysis, we considered continuous and binary outcome variables, with identity and logit links for $g$, respectively. 
For each bootstrap sample, the significance of the estimated vector of weights was evaluated through the significance $(p \leq .05)$ of $\hat{\beta}_{1}$, which corresponds to the parameter estimate for the weighted index. The weighted quantile score was then estimated as $W Q S=\sum_{i=1}^{c} \bar{w}_{i} q_{i}$, where $\bar{w}_{i}=\frac{1}{n_{B}} \sum_{j=1}^{n_{B}} w_{i j}$, and $n_{B}$ is defined as the number of bootstrap samples in which $\hat{\beta}_{1}$ was significant. Finally, the significance of $W Q S$ was assessed using the validation data set and the model

$$
g(\mu)=\beta_{0}+\beta_{1} W Q S+\mathbf{z}^{\prime} \boldsymbol{\varphi}
$$

Simultaneous estimation of the unknown weights and parameters was achieved through the use of an optimization algorithm that maximized the nonlinear function in equation (3.1), subject to the linear constraint $\sum_{i=1}^{c} w_{i}=1$ and the bounds $w_{i} \in[0,1]$. The nonlinear optimization was performed in R using the function solnp found in the package Rsolnp. The algorithm employed belongs to the class of indirect solvers, and implements the augmented Lagrange multiplier method with a sequential quadratic programming interior algorithm.

For each of the 100 simulated datasets for each set of simulation conditions (correlation pattern and outcome correlation), we performed WQS regression on the full data set (adjusted for study location) and separately at each site. The ranks used in WQS regression were calculated within each site for the site specific analyses, and overall for the site adjusted analysis of the full data set. The process was performed twice, once using the continuous outcome variable, and once using the binary outcome variable. Therefore, for each set of conditions a total of five indices (four site-specific indices and one full-study index) were estimated for each outcome variable. 
The median number of correctly and incorrectly selected chemicals was calculated for each of the five indices across the 100 simulated studies. A chemical was identified as selected if it received a weight of at least 0.05 . The significance of the five estimated indices in their respective validation data sets was also examined.

\subsubsection{Comparison of WQS regression with lasso, adaptive lasso, and elastic net}

Modern methods that address collinearity and high-dimensionality (e.g., lasso, elastic net), have been demonstrated to be less accurate in the selection of potentially harmful chemicals compared with WQS regression (Carrico et al., 2014). To further assess the use of shrinkage regression models for evaluating effects of chemical exposures, we fitted lasso (Tibshirani, 1996), adaptive lasso (Zou, 2006), and elastic net (Zou et al., 2005) models to the 100 training data sets (of size $\left.N_{t}=500\right)$ for each set of conditions (correlation pattern and outcome association) for both the continuous and binary response variables. In an effort to most closely parallel the site-adjusted model used in the estimation of WQS weights in these overall data sets, indicator variables for site were included in the lasso, adaptive lasso, and elastic net models, but were not subjected to the penalty (i.e., these variables were forced to remain in the model). The penalized regressions were performed in $\mathrm{R}$ using the cv.glmnet and glmnet functions in the glmnet package (Friedman et al., 2010). For the lasso and adaptive lasso models, the tuning parameters were chosen using cross-validation and the one standard error rule (Breiman et al., 1984). For the elastic net models, a grid search was performed using cross-validation, with the elastic net mixing parameter allowed to vary from 0 to 1 .

For the lasso, adaptive lasso and elastic net methods, chemicals related to the outcome variable were identified as correctly chosen if they were retained in the model with a positive coefficient, while chemicals not related to the outcome variable were identified as incorrectly chosen if they 
were retained in the model. The median and IQR for the number of correctly and incorrectly selected chemicals were reported, and the three methods were compared in terms of sensitivity and specificity.

\subsection{Results}

\subsubsection{Sensitivity and specificity of WQS regression}

The median number of correctly and incorrectly chosen chemicals across 100 samples for each setting is displayed in Tables 3.2 and 3.3, respectively. When association with the outcome was strong $(r=0.3)$, the estimated weights for sites Detroit, Iowa, and Seattle performed at least as well as the weights estimated using the full data set, in terms of both sensitivity and specificity. Based on the weights for these three sites, WQS regression correctly chose all seven chemicals at least half of the time (median value) for both the continuous and binary responses, regardless of the correlation pattern among predictors. The weights estimated from the full study population also correctly chose all seven chemicals at least half of the time (median value), with the exception of the setting in which the correlation among predictors was strongest (65\% of observed site-specific correlations) and the outcome was continuous (median of six correctly chosen chemicals). With respect to specificity, when outcome correlation was strong, the weights for sites Detroit, Iowa, and Seattle, and the weights for the full study population had a median value of zero incorrectly chosen chemicals at all settings. When association with the outcome was weak $(r=0.1)$, the weights estimated from the full data set may have slightly improved sensitivity, as the median number of correctly chosen chemicals for the overall analysis was often greater by one chemical (as compared to the median number correctly selected in Detroit, Iowa, and Seattle). This one chemical increase in sensitivity was seen across all correlation patterns for the binary outcome variable, and in the case of moderate correlation (65\% of that 
observed) among chemicals for the continuous variable. Similarly, the weights estimated from the full data set may have slightly increased specificity when outcome association was weak, as the site-specific weights tended to incorrectly choose one additional chemical.

From the results for the Los Angeles site, all four chemicals were correctly selected at least half of the time for each setting, but the number of incorrectly chosen chemicals ranged from two to four across the different settings. Because fewer chemicals (four) were set to be associated with the outcome within this site, it may have been advantageous to define a criterion for chemical selection unique to this site.

Table 3.2: Median [IQR] number of correctly chosen chemicals for the five WQS indices Weak Association with Strong Association

Outcome with Outcome

\begin{tabular}{|c|c|c|c|c|c|c|c|}
\hline & & \multicolumn{3}{|c|}{ Correlation Pattern } & \multicolumn{3}{|c|}{ Correlation Pattern } \\
\hline Cont. Outcome & Max & 0.65 & 0.30 & 0.01 & 0.65 & 0.30 & 0.01 \\
\hline WQS $_{\text {Detroit }}$ & 7 & $5[5,6]$ & $6[5,6]$ & $6[5,6]$ & $7[7,7]$ & $7[7,7]$ & $7[7,7]$ \\
\hline WQS $_{\text {Iowa }}$ & 7 & $5[5,6]$ & $6[5,6]$ & $6[5,6]$ & $7[7,7]$ & $7[7,7]$ & $7[7,7]$ \\
\hline $\mathrm{WQS}_{\mathrm{LA}}$ & 4 & $4[3,4]$ & $4[3,4]$ & $4[3,5]$ & $4[4,4]$ & $4[4,4]$ & $4[4,4]$ \\
\hline $\mathrm{WQS}_{\text {Seattle }}$ & 7 & $5[5,6]$ & $6[5,6]$ & $6[5,6]$ & $7[7,7]$ & $7[7,7]$ & $7[7,7]$ \\
\hline WQS $_{\text {Full }}$ & 7 & $6[5,6]$ & $6[6,7]$ & $6[6,6]$ & $6[6,7]$ & $7[7,7]$ & $7[7,7]$ \\
\hline \multicolumn{8}{|l|}{ Binary Outcom } \\
\hline $\mathrm{WQS}_{\mathrm{De}}$ & 7 & $5[4,5]$ & $5[4,6]$ & $5[4,6]$ & $7[6,7]$ & $7[7,7]$ & $7[7,7]$ \\
\hline $\mathrm{WQS}_{\text {Iowa }}$ & 7 & $5[4,5]$ & $5[4,6]$ & $5[4,6]$ & $7[7,7]$ & $7[7,7]$ & $7[7,7]$ \\
\hline WQS $_{\text {LA }}$ & 4 & $4[3,4]$ & $4[3,4]$ & $4[3,4]$ & $4[4,4]$ & $4[4,4]$ & $4[4,4]$ \\
\hline WQS & 7 & $5[4,5]$ & $5[4,5]$ & $5[4,5]$ & $7[6,7]$ & $7[7,7]$ & $7[7,7]$ \\
\hline $\mathrm{WQS}_{\text {Full }}$ & 7 & $6[6,6]$ & $6[6,7]$ & $6[6,7]$ & $7[7,7]$ & $7[7,7]$ & $7[7,7]$ \\
\hline
\end{tabular}

Cont. Outcome denotes the continuous outcome variable 
Table 3.3: Median [IQR] number of incorrectly chosen chemicals for the five WQS indices Weak Association with Strong Association with

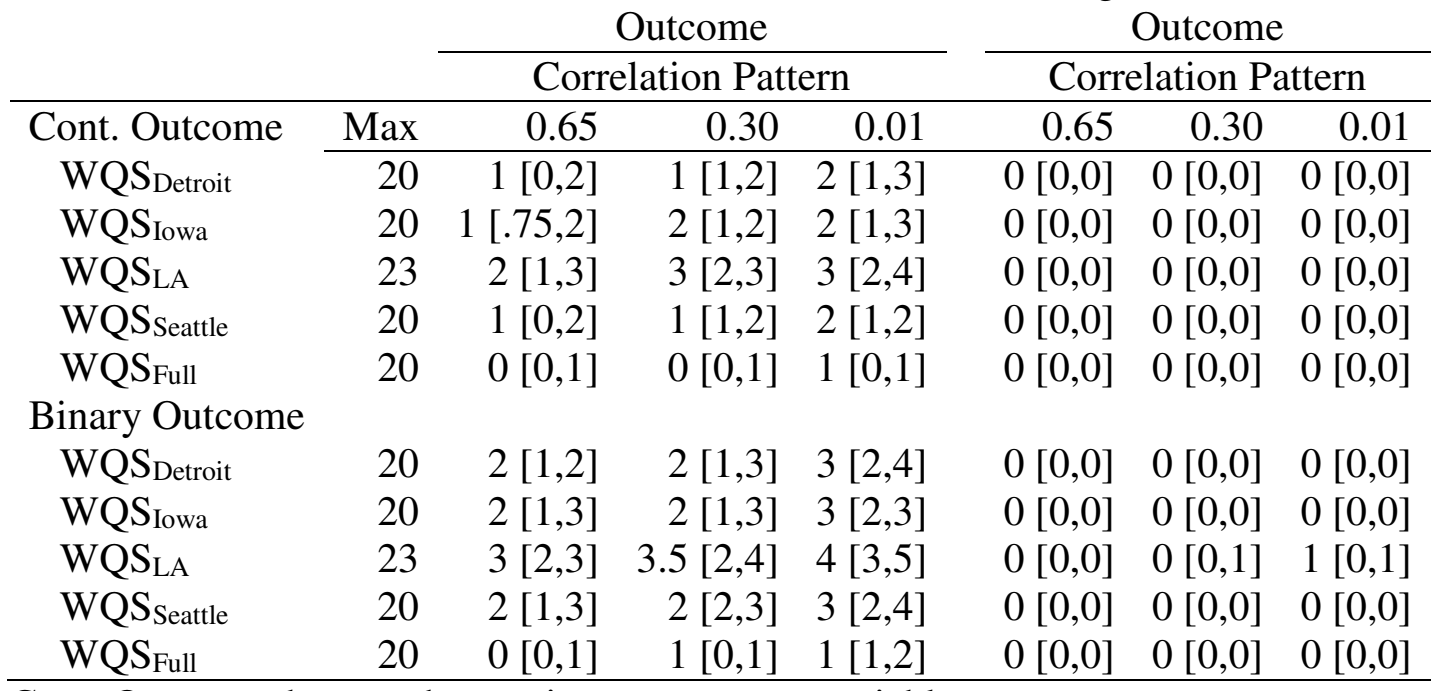

Cont. Outcome denotes the continuous outcome variable

In summary, WQS regression had good sensitivity and specificity at all settings for the sitespecific and overall analysis for both the continuous and binary outcome variable. Performance of the site-specific analyses was comparable to that of the overall analysis. We caution against overinterpretation of a one or two chemical difference in sensitivity and/or specificity, as any perceived improved performance for the overall analyses in comparison to the site-specific analyses may be a result of the 4-fold increase in sample size in the estimation data set for the index derived from the full study population. Furthermore, the results presented in this analysis are dependent upon chemical selection as defined by a minimum value of 0.05 for the estimated chemical weight. It was decided a priori that a chemical must receive at least $5 \%$ of the weights to be considered important. While this may be reasonably applied in practice, the method for best choosing a cut-off value is still an open area of research. The choice of cutoff value may be affected by number of chemicals, correlation structure, signal strength, etc. 
With respect to chemical selection, we generally expect to see an increase in sensitivity and a decrease in specificity as the threshold weight for chemical selection is lowered. Figure 3.4 shows modified receiver operating curves (ROC) for the three different correlation structures among the chemicals in the setting of weak association with the (a) continuous outcome and (b) binary outcome, with the cutoff weight for chemical selection varied. The true positive rate (sensitivity) was calculated as the average percentage of correctly selected chemicals across the 100 simulations, and the false positive rate (1-sensitivity) was calculated as the average percentage of incorrectly selected chemicals across the 100 simulations. As the cutoff weight for chemical selection is lowered, we see an increase in both the average true and false positive rates as expected. The a priori chosen cutoff of 0.05 (i.e., $5 \%$ of the total chemical weights) performed well regardless of the level of correlation among chemicals or strength of association with outcome. When association with the continuous outcome was weak (Figure 3.4, left) the average false positive rate for the cutoff weight of 0.05 ranged from $2.3 \%$ to $4.5 \%$ across the correlation structures, while the average true positive rate ranged from $83.9 \%$ to $85.6 \%$. Similarly, for the binary outcome (Figure 3.4, right) the average false positive rate for the cutoff weight of 0.05 ranged from $2.6 \%$ to $6.3 \%$ across the correlation structures, while the average true positive rate ranged from $85.6 \%$ to $89.9 \%$. Finally, when association with the outcome was strong (results not shown) the average false positive rate for the cutoff weight of 0.05 was at most $0.05 \%$, while the average true positive rate was at least $92.6 \%$ across the three correlation structures and both the continuous and binary outcomes. 

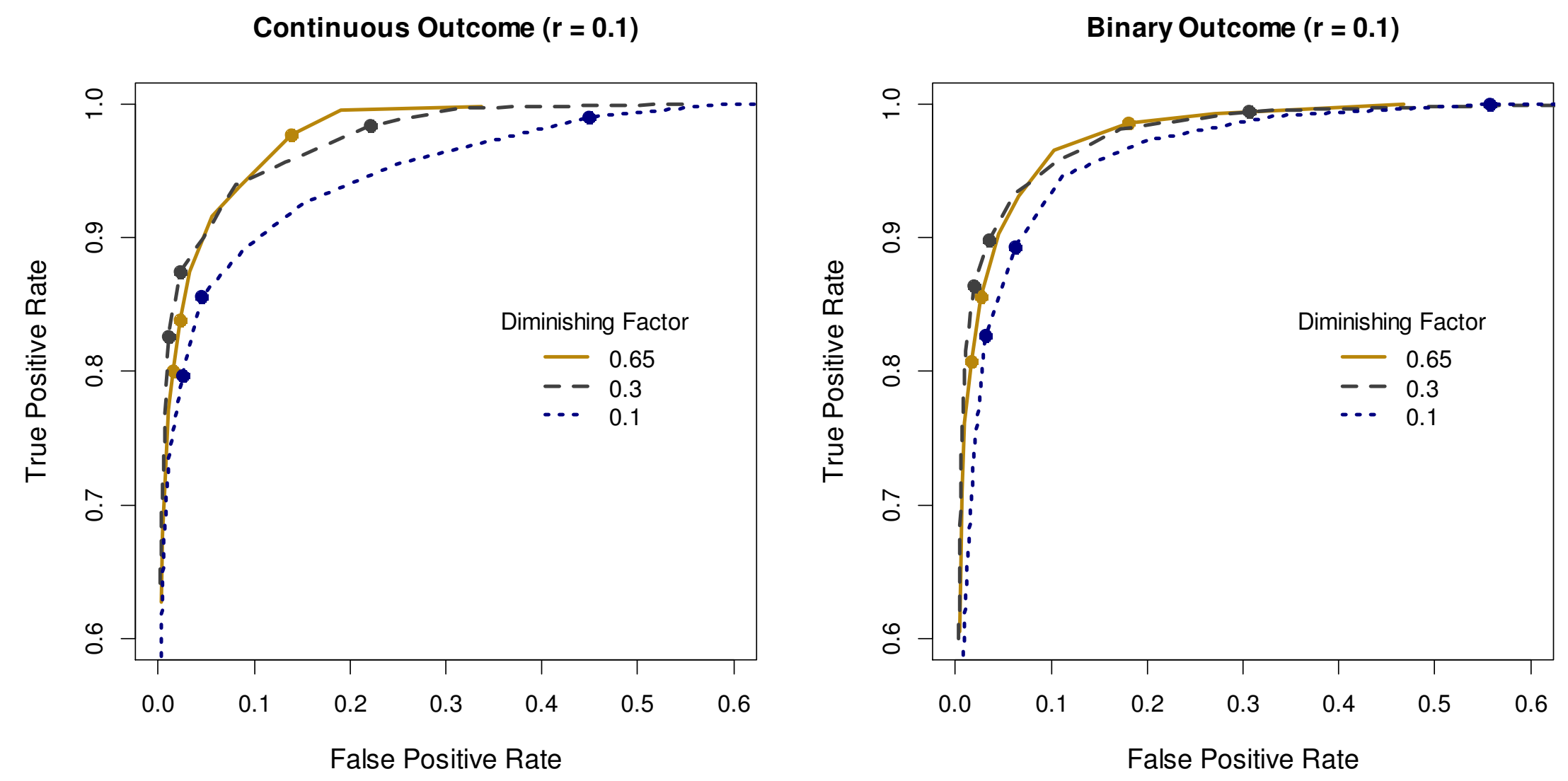

Figure 3.4: Modified receiver operating curves for the WQS index derived from the full study population with varying weight thresholds for chemical selection. The true positive rate was calculated as the average percentage of correctly selected chemicals (i.e., the average number of correctly selected chemicals divided by 7) over the 100 simulations, while the false positive rate was calculated as the average percentage of incorrectly selected chemicals (i.e., the average number of incorrectly selected chemicals divided by 20). The points on each line represent (from left to right) the average true and false positive rates for weight thresholds of $0.01,0.05$, and 0.06. Diminishing factor refers to the level of correlation among the chemicals (i.e., the amount by which the observed chemical correlations were diminished). 


\subsubsection{Distribution of WQS regression weights}

In practice, we also look at the distributions of the weights in deciding which chemicals are important. Figure 3.5 shows the distribution of the average weights across the 100 simulated samples for the seven chemicals assumed to be associated with outcome for each of the five indices. The plots focus on the setting in which there was weak correlation with the continuous outcome for (a) moderate correlation (65\% of that observed) among chemicals, and (b) correlation diminished to $1 \%$ of that observed. For both correlation structures, WQS appropriately placed considerable weight on the true bad actors, and also placed negligible weight on chemicals uncorrelated with the outcome. The latter is demonstrated by the near zero weight placed on X8, X9, and X19 by the Los Angeles index. Also, as correlation among chemicals was diminished, reliability of the weights improved, as evident by the narrowed distributions in (b).

When comparing the weights from the different indices, the index for Los Angeles tended to place greater emphasis on chemicals X5, X20, X24, and X27 compared with the other sites. This is likely due to the fact that these four chemicals were the only true bad actors in this site, and thus the weights as a whole were divided over fewer components. Additionally, the weights for the full study population analysis seem to demonstrate an averaging effect across the sites, as they appear to shift downward for the chemicals that were unassociated with outcome in Los Angeles (X8, X9, X19). For the chemicals associated with outcome in all four sites (X5, X20, $\mathrm{X} 24$, and X27), the weights estimated by the overall analysis were slightly higher than those estimated in site-specific analysis. This may be attributable to the increased power (greater sample size) of the overall analysis. 

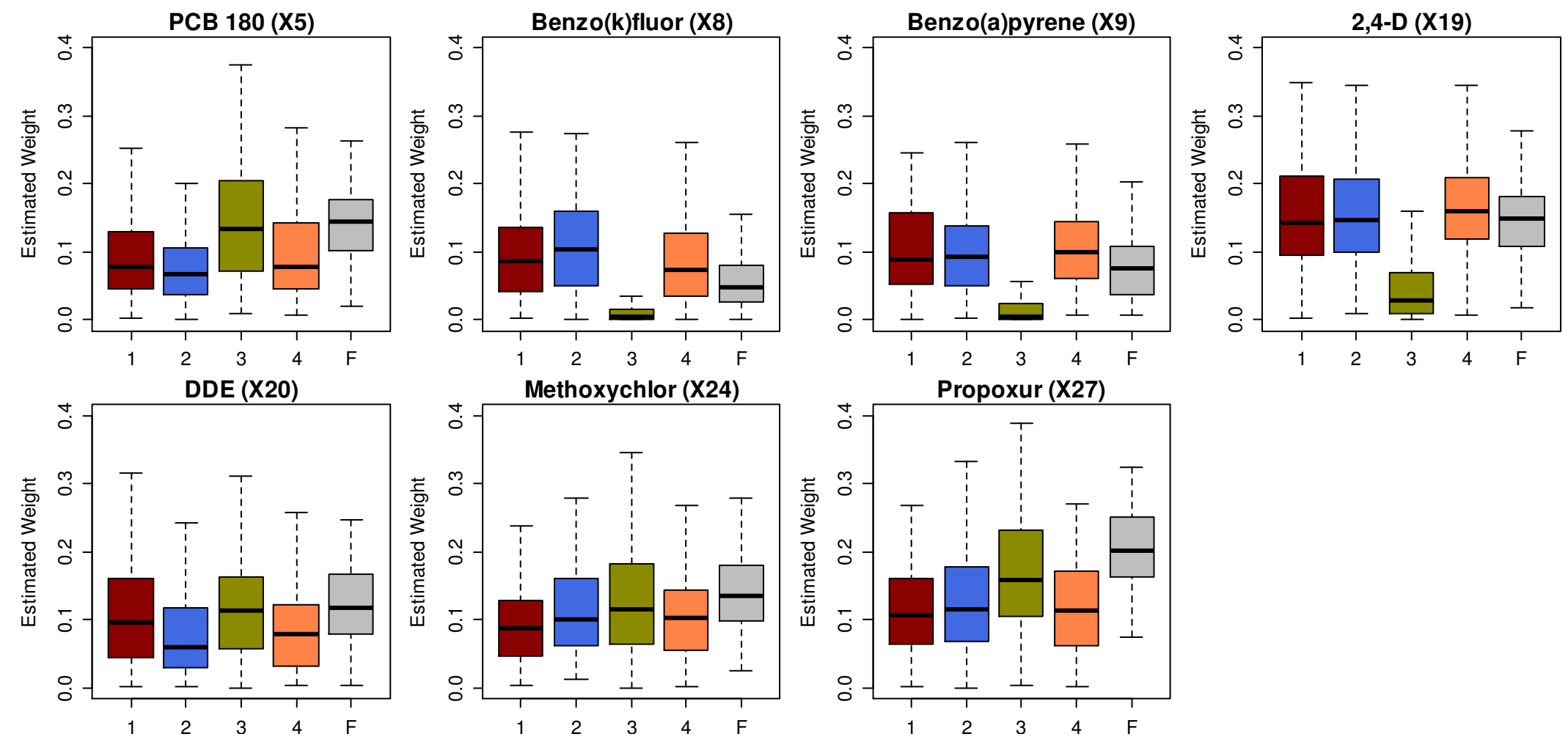

a) Correlation among chemicals diminished to $65 \%$ of the observed site-specific correlation structures. 

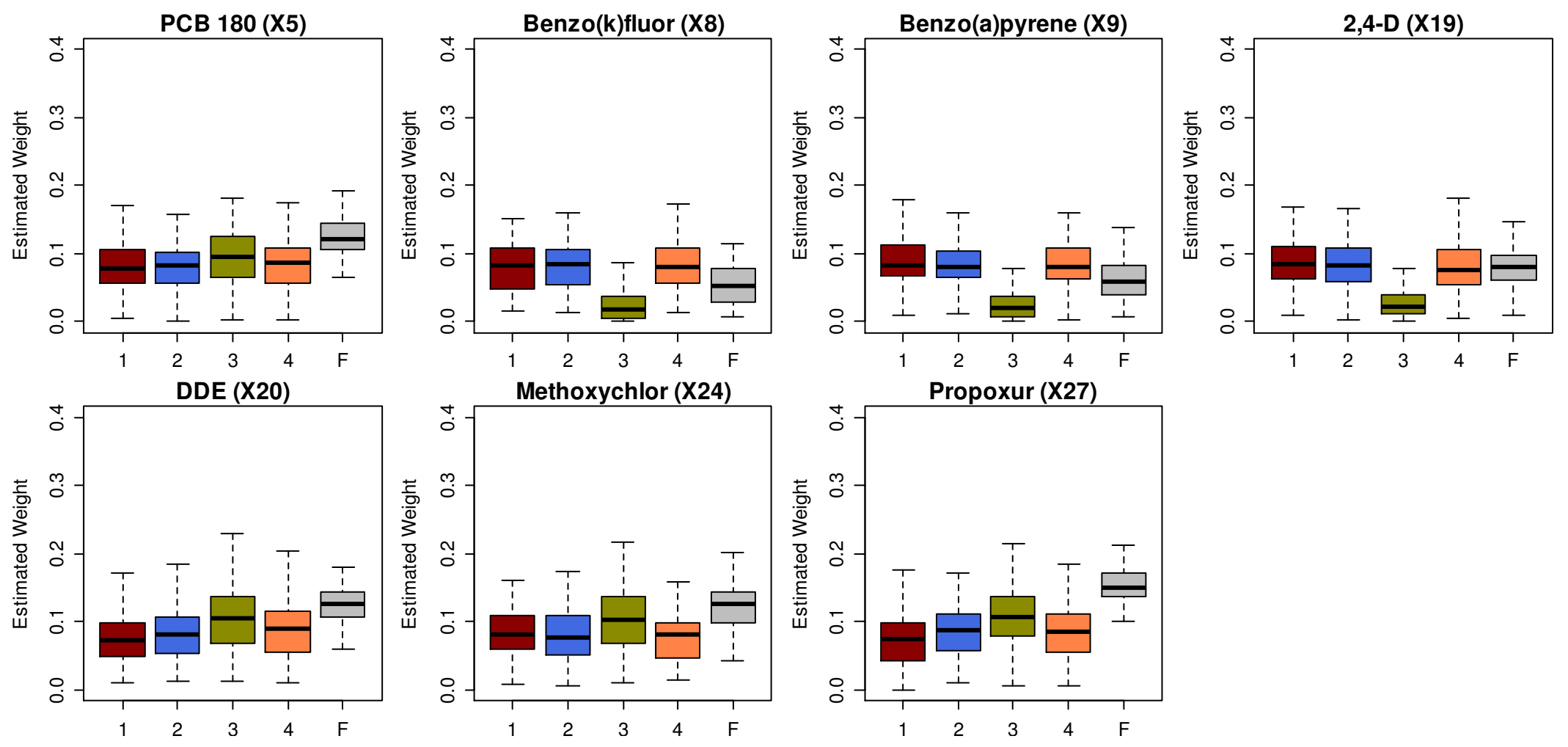

b) Correlation among chemicals diminished to $1 \%$ of the observed site-specific correlation structures

Figure 3.5: Distribution of WQS index weights for the five WQS indices across the 100 simulated data sets for the seven chemicals weakly associated $(r=0.1)$ with the continuous outcome for a) moderate correlation ( $65 \%$ of that observed) among chemicals, and $b)$ correlation among chemicals diminished to $1 \%$ of that observed.

Note: Site 1 = Detroit, 2 = Iowa, 3 = Los Angeles, 4 = Seattle, $F$ = full study population 
When strongly correlated with the continuous outcome (data not shown), the findings were consistent with those discussed above, but the important chemicals (true bad actors) tended to receive higher average weights. With respect to the binary outcome (data not shown), the findings were again analogous.

\subsubsection{Power of WQS regression}

The estimated weights were applied to the validation data sets and significance was assessed

through the $\hat{\beta}_{1}$ parameter. The results of the significance tests across the simulated data sets are summarized in Tables 3.4 and 3.5. The average parameter estimates were all positively associated with outcome, suggesting that increased body burden (as estimated by the WQS index) was associated with increased risk. Overall power increased, as expected, as the association with the outcome variable strengthened and correlation among chemicals diminished. Additionally, the indices estimated using the continuous outcome variable had greater power in comparison to those using the binary outcome variable. Finally, the indices for the Los Angeles site exhibited lower power in comparison to the other indices. This is likely because the set of predictor variables as a whole were contributing less information, as fewer chemicals were set to be associated with outcome. This became more pronounced, again as expected, when correlation among chemicals strengthened and association with outcome was weakened. 
Table 3.4: Summary of testing results for the five WQS indices across 100 simulated examples for the continuous outcome variable.

\begin{tabular}{|c|c|c|c|c|c|c|}
\hline \multirow[b]{2}{*}{ Correlation } & \multicolumn{3}{|c|}{ Weak Association with Outcome } & \multicolumn{3}{|c|}{ Strong Association with Outcome } \\
\hline & $\hat{\beta}_{1}$ & $95 \% \mathrm{CI}$ & $\%$ sig. & $\hat{\beta}_{1}$ & $95 \% \mathrm{CI}$ & $\%$ sig. \\
\hline \multicolumn{7}{|l|}{$65 \%$} \\
\hline $\mathrm{WQS}_{\text {Detroit }}$ & 0.27 & $(0.11,0.44)$ & 91 & 1.00 & $(0.87,1.13)$ & 100 \\
\hline $\mathrm{WQS}_{\text {Iowa }}$ & 0.29 & $(0.12,0.46)$ & 89 & 1.07 & $(0.94,1.21)$ & 100 \\
\hline $\mathrm{WQS}_{\mathrm{LA}}$ & 0.18 & $(0.00,0.35)$ & 58 & 0.69 & $(0.55,0.83)$ & 100 \\
\hline $\mathrm{WQS}_{\text {Seattle }}$ & 0.31 & $(0.13,0.50)$ & 96 & 1.07 & $(0.93,1.21)$ & 100 \\
\hline WQS Full & 0.30 & $(0.20,0.40)$ & 100 & 0.94 & $(0.87,1.02)$ & 100 \\
\hline \multicolumn{7}{|l|}{$30 \%$} \\
\hline $\mathrm{WQS}_{\text {Detroit }}$ & 0.39 & $(0.18,0.60)$ & 96 & 1.31 & $(1.16,1.45)$ & 100 \\
\hline $\mathrm{WQS}_{\text {Iowa }}$ & 0.40 & $(0.18,0.61)$ & 99 & 1.35 & $(1.19,1.51)$ & 100 \\
\hline $\mathrm{WQS}_{\mathrm{LA}}$ & 0.26 & $(0.06,0.45)$ & 75 & 0.87 & $(0.74,1.00)$ & 100 \\
\hline WQS $_{\text {Seattle }}$ & 0.41 & $(0.15,0.66)$ & 94 & 1.35 & $(1.20,1.50)$ & 100 \\
\hline $\mathrm{WQS}_{\text {Full }}$ & 0.40 & $(0.29,0.51)$ & 100 & 1.19 & $(1.11,1.27)$ & 100 \\
\hline \multicolumn{7}{|l|}{$1 \%$} \\
\hline $\mathrm{WQS}_{\text {Detroit }}$ & 0.64 & $(0.32,0.96)$ & 99 & 1.91 & $(1.72,2.11)$ & 100 \\
\hline $\mathrm{WQS}_{\text {Iowa }}$ & 0.62 & $(0.28,0.97)$ & 97 & 1.94 & $(1.74,2.14)$ & 100 \\
\hline $\mathrm{WQS}_{\mathrm{LA}}$ & 0.44 & $(0.17,0.70)$ & 87 & 1.28 & $(1.05,1.51)$ & 100 \\
\hline $\mathrm{WQS}_{\text {Seattle }}$ & 0.66 & $(0.37,0.96)$ & 100 & 1.92 & $(1.74,2.10)$ & 100 \\
\hline $\mathrm{WQS}_{\text {Full }}$ & 0.58 & $(0.45,0.72)$ & 100 & 1.64 & $(1.51,1.76)$ & 100 \\
\hline
\end{tabular}

$\hat{\beta}_{1}$ is given as the average across 100 simulated data sets; CI denotes confidence interval;

$\%$ sig. denotes the $\%$ of simulated examples in which $\hat{\beta}_{1}$ was significant $(\alpha=0.05)$. 
Table 3.5: Summary of testing results for the five WQS indices across 100 simulated examples for the binary outcome variable.

\begin{tabular}{|c|c|c|c|c|c|c|}
\hline \multirow[b]{2}{*}{ Correlation } & \multicolumn{3}{|c|}{ Weak Association with Outcome } & \multicolumn{3}{|c|}{ Strong Association with Outcome } \\
\hline & $\hat{\beta}_{1}$ & $95 \% \mathrm{CI}$ & $\%$ sig. & $\hat{\beta}_{1}$ & $95 \% \mathrm{CI}$ & $\%$ sig. \\
\hline \multicolumn{7}{|l|}{$65 \%$} \\
\hline WQS $_{\text {Detroit }}$ & 0.43 & $(0.07,0.78)$ & 61 & 2.00 & $(1.47,2.53)$ & 100 \\
\hline $\mathrm{WQS}_{\text {Iowa }}$ & 0.45 & $(0.01,0.89)$ & 61 & 2.18 & $(1.70,2.66)$ & 100 \\
\hline WQS $_{\mathrm{LA}}$ & 0.25 & $(-0.14,0.65)$ & 28 & 1.28 & $(0.91,1.64)$ & 100 \\
\hline WQS $_{\text {Seattle }}$ & 0.48 & $(0.06,0.89)$ & 67 & 2.16 & $(1.65,2.66)$ & 100 \\
\hline $\mathrm{WQS}_{\text {Full }}$ & 0.51 & $(0.31,0.72)$ & 100 & 1.99 & $(1.73,2.26)$ & 100 \\
\hline \multicolumn{7}{|l|}{$30 \%$} \\
\hline $\mathrm{WQS}_{\text {Detroit }}$ & 0.65 & $(0.19,1.10)$ & 84 & 2.81 & $(2.11,3.52)$ & 100 \\
\hline WQS $_{\text {Iowa }}$ & 0.64 & $(0.10,1.17)$ & 74 & 2.97 & $(2.34,3.60)$ & 100 \\
\hline $\mathrm{WQS}_{\mathrm{LA}}$ & 0.35 & $(-0.08,0.78)$ & 31 & 1.70 & $(1.26,2.14)$ & 100 \\
\hline $\mathrm{WQS}_{\text {Seattle }}$ & 0.62 & $(0.08,1.17)$ & 74 & 2.97 & $(2.32,3.62)$ & 100 \\
\hline $\mathrm{WQS}_{\text {Full }}$ & 0.70 & $(0.45,0.95)$ & 100 & 2.67 & $(2.39,2.96)$ & 100 \\
\hline \multicolumn{7}{|l|}{$1 \%$} \\
\hline $\mathrm{WQS}_{\text {Detroit }}$ & 1.00 & $(0.29,1.71)$ & 84 & 4.82 & $(3.92,5.72)$ & 100 \\
\hline $\mathrm{WQS}_{\text {Iowa }}$ & 0.97 & $(0.26,1.67)$ & 82 & 4.93 & $(3.95,5.91)$ & 100 \\
\hline $\mathrm{WQS}_{\mathrm{LA}}$ & 0.68 & $(0.09,1.27)$ & 61 & 2.67 & $(1.95,3.38)$ & 100 \\
\hline WQS $_{\text {Seattle }}$ & 1.03 & $(0.39,1.67)$ & 90 & 4.95 & $(3.95,5.96)$ & 100 \\
\hline $\mathrm{WQS}_{\text {Full }}$ & 1.10 & $(0.77,1.43)$ & 100 & 4.21 & $(3.70,4.72)$ & 100 \\
\hline
\end{tabular}

$\hat{\beta}_{1}$ is given as the average across 100 simulated data sets; CI denotes confidence interval;

$\%$ sig. denotes the $\%$ of simulated examples in which $\hat{\beta}_{1}$ was significant $(\alpha=0.05)$.

\subsubsection{Comparison of WQS regression with lasso, adaptive lasso, and elastic net}

Lasso, adaptive lasso, and elastic net regression were performed on only the full study population for the both the binary and continuous outcomes for the six different simulation settings. The median number of correctly and incorrectly chosen chemicals for the lasso, adaptive lasso, elastic net, and WQS regression models across 100 samples are given in Tables 3.6 and 3.7. When the predictors were strongly associated with the outcome, WQS and the traditional shrinkage methods demonstrated a high degree of sensitivity for both the continuous and binary response variables, regardless of the level of correlation among predictors. For the continuous outcome, each of the shrinkage methods correctly selected all seven chemicals at least half of the time, while WQS regression correctly selected a median of at least six of the seven chemicals. In 
the case of the binary outcome, each of the methods correctly chose all seven chemicals at least half of the time.

When considering the setting of weak association among the predictors and the outcome, WQS regression correctly selected six of the seven chemicals at least half of the time, for both the continuous and binary outcomes, regardless of the level of correlation among the predictors. Similarly, the median number of correctly chosen chemicals for elastic net ranged between six and seven for both the continuous and binary response. In contrast, lasso and adaptive lasso demonstrated diminished sensitivity, with the median number of correctly chosen chemicals ranging from four to seven for the continuous outcome and three to five for the binary outcome.

While the penalized regression models may have exhibited sensitivity that was comparable to that of WQS regression in several settings, the sensitivity of these traditional shrinkage methods was often overshadowed by their lack of specificity. WQS regression was highly specific, choosing at most a median of one incorrect chemical, regardless of the degree of correlation among predictors, and regardless of the strength of association with the response. In contrast, as correlation among the chemicals increased, the penalized regression methods demonstrated a loss of specificity. In particular, when the predictors were strongly associated with the response (both continuous and binary), the penalized regression models chose a median of at least 14 incorrect chemicals in the presence of moderate or mild correlation among chemicals. Most notably, the lasso and elastic net had a tendency to select almost all of the chemicals when the chemicals were moderately correlated, and strongly associated with the response. The relatively low specificity of these shrinkage methods appears to limit their role in risk evaluation of environmental chemical mixtures. 
Table 3.6: Median [IQR] number of correctly selected chemicals for lasso, adaptive lasso, elastic net, and WQS regression for the full study population.

\begin{tabular}{|c|c|c|c|c|c|c|}
\hline & \multicolumn{3}{|c|}{$\begin{array}{l}\text { Weak Association } \\
\text { with Outcome }\end{array}$} & \multicolumn{3}{|c|}{$\begin{array}{l}\text { Strong Association } \\
\text { with Outcome }\end{array}$} \\
\hline & \multicolumn{3}{|c|}{ Correlation } & \multicolumn{3}{|c|}{ Correlation } \\
\hline Continuous Outcome & 0.65 & 0.30 & 0.01 & 0.65 & 0.30 & 0.01 \\
\hline Lasso & $7[6,7]$ & $4[3,6]$ & $5[4,6]$ & $7[7,7]$ & $7[7,7]$ & $7[7,7]$ \\
\hline Adaptive Lasso & $6[6,7]$ & $4[3,5]$ & $5[4,6]$ & $7[7,7]$ & $7[7,7]$ & $7[7,7]$ \\
\hline Elastic Net & $7[6,7]$ & $6[5,7]$ & $6[5,7]$ & $7[7,7]$ & $7[7,7]$ & $7[7,7]$ \\
\hline WQS $_{\text {Full }}$ & $6[5,6]$ & $6[6,7]$ & $6[6,6]$ & $6[6,7]$ & $7[7,7]$ & $7[7,7]$ \\
\hline \multicolumn{7}{|l|}{ Binary Outcome } \\
\hline Lasso & $5[3,6]$ & $3[0,5]$ & $3[0,5]$ & $7[7,7]$ & $7[7,7]$ & $7[7,7]$ \\
\hline Adaptive Lasso & $5[4,6]$ & $3[1,5]$ & $3[2,5]$ & $7[7,7]$ & $7[7,7]$ & $7[7,7]$ \\
\hline Elastic Net & $7[6,7]$ & $7[5.75,7]$ & $6[4,7]$ & $7[7,7]$ & $7[7,7]$ & $7[7,7]$ \\
\hline $\mathrm{WQS}_{\text {Full }}$ & $6[6,6]$ & $6[6,7]$ & $6[6,7]$ & $7[7,7]$ & $7[7,7]$ & $7[7,7]$ \\
\hline
\end{tabular}

Table 3.7: Median [IQR] number of incorrectly selected chemicals for lasso, adaptive lasso, elastic net, and WQS regression for the full study population.

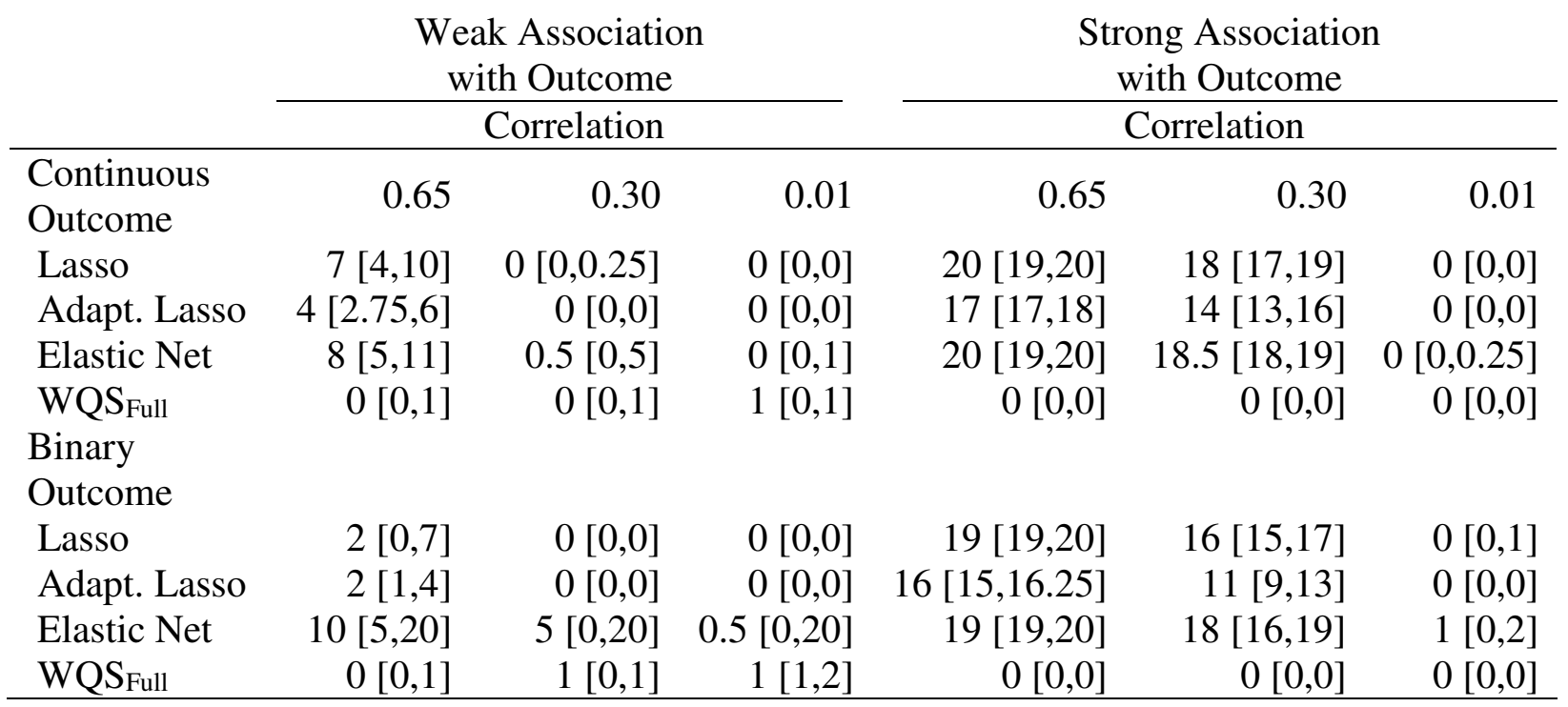

\subsection{Discussion and Conclusion}

WQS regression demonstrated good sensitivity and specificity for both site-specific models and the full study population model across a variety of conditions considered in this study. WQS adequately detected important predictors, while simultaneously placing negligible weight on chemicals unassociated with outcome, for both continuous and binary response variables. 
Additionally, the WQS index was significantly and positively associated with the outcome when tested in the validation data sets, and generally demonstrated good power. Results improved as correlation among chemicals diminished and association with the outcome strengthened. In comparison to the shrinkage regression methods of lasso and elastic net, WQS performed well for sensitivity and specificity, while the lasso and elastic-net models exhibited good sensitivity but poor specificity. The shrinkage methods had a tendency to incorrectly identify a large number of components, especially in the case of strong association with the outcome. This suggests that these methods may be limited for use in risk assessment, as they are unable to discern which chemicals are unassociated with health risk.

The WQS index weights for the full study population demonstrated an averaging effect, suggesting that chemical weights estimated in an overall analysis may not be representative of the true bad actors within a site. Three chemicals were deemed unassociated with outcome in the Los Angeles site, as they were not present in high enough concentrations to satisfy the imposed definition of health risk. The overall analysis consistently identified these three chemicals unassociated with outcome in Los Angeles as bad actors. While this is representative of the data as a whole (these chemicals were set as truly bad actors in three of the four sites), it is not an accurate representation of the chemicals posing risk in Los Angeles. Additionally, the average weights assigned to these three chemicals by the index in the full study population were lower in comparison to the weights assigned by the indices in Detroit, Iowa, and Seattle. The nonassociation in Los Angeles therefore seems to result in an underestimation (by the full index) of the importance of these chemicals in the sites in which they truly were bad actors.

With the goal of identifying chemicals that pose a significant health risk, it is of great importance to consider the toxicological principle that "the dose makes the poison," especially given that 
exposure patterns are spatially varying. Although a chemical may not be present in high enough concentrations to pose a health risk in one location, it may still pose a significant health risk at other locations. Though limited, these simulation studies suggest that use of an overall index may overstate the importance of a chemical in sites where the concentration is too low to constitute risk, and may understate the importance of a chemical in locations where it is present in concentrations that are high enough to adversely affect health.

The simulation studies conducted in this analysis were largely reflective of the exposure patterns observed in the original NCI-SEER NHL study, incorporating the exposure concentrations and the complex correlation among chemicals on a site-specific basis. However, simulation of the data utilized Cholesky decomposition, which required that the covariance matrices be positive definite. As a result, the simulations only incorporated (at most) $65 \%$ of the observed correlation structures, as the covariance matrices became singular if they were any less diminished. Other studies have used methods such as ridging to allow the correlation to be preserved or even inflated (Carrico, 2013), which should be considered in future work. We expect that as correlation among chemicals increases, one will encounter cases in which WQS may not perform as well as was seen in this study. Finally, in the general context of risk assessment, it is a limitation that the observed chemical concentrations in the NCI-SEER NHL study were external measures of exposures, as what is found in house dust may not be truly reflective of an individual's absorption or ingestion of chemicals. 


\section{Chapter 4}

Evaluating Geographically Weighted Regression Models for Environmental Chemical Risk Analysis

\subsection{Introduction}

Humans are exposed to mixtures of chemicals that may be influential for cancer risk. For example, risk of non-Hodgkin lymphoma (NHL) is suspected to be associated with several chemicals through environmental or occupational routes of exposure, and geographic variation in NHL rates suggests the importance of environmental risk factors (Hartge et al. 2006). Positive associations have been found with persistent organochlorine chemicals, including polychlorinated biphenyls (PCBs) (Engel et al. 2007), particularly PCB congener 180 (Colt et al. 2005; De Roos et al. 2005; Morton et al. 2008), and dichlorodiphenyldichloroethylene (DDE) (Colt et al. 2005; Engel L et al. 2007).

Environmental exposure patterns are typically complex with inherent correlations among cooccurring chemicals or their metabolites (Czarnota et al., 2014). For example, many PCB congeners exhibit a high degree of correlation. Important questions in the analysis of mixtures include whether and how the health effect of one chemical should be adjusted for other chemicals present, even when those chemicals are highly correlated. Furthermore, the relationship between environmental chemicals and health effects (e.g., cancer risk) is not always constant across a study area (Czarnota et al., 2014). Exposure levels may be different spatially due to environmental factors. For example, pesticide levels measured in house dust may be 
higher in agricultural communities (e.g., in Iowa) or those in temperate climates where more pesticides are applied throughout the year (e.g., Los Angeles) compared to urban locations (e.g., Detroit). Acknowledging the principle that "the dose makes the poison", the risk of adverse health effects such as NHL is greater in regions where exposure is higher. Thus, environmental health models that account for these spatially changing exposure/risk regions can be informative.

Models with spatially varying coefficients include geographically weighted regression (GWR; Fortheringham et al, 2002), which is similar to local linear regression (e.g., Cleveland, 1979; Hastie et al., 2001; Loader, 1999) in that both methods use a kernel function to calculate weights that are applied to observations in a series of local weighted regression models. One issue with GWR is that GWR models have been found to be impacted by local collinearity (Wheeler and Tiefelsdorf, 2005; Wheeler, 2007; Wheeler and Calder, 2008; Wheeler, 2009; Páez et al., 2011). Local collinearity in weighted explanatory variables can lead to GWR coefficient estimates that are correlated locally and across space, have inflated variances, and are at times counterintuitive and contradictory in sign to the global regression estimates, i.e., evidence of the reversal paradox (Wheeler, 2007; Tu et al, 2008).

To illustrate, Wheeler and Tiefelsdorf (2005) highlighted the issue of collinearity in GWR in a simple model to explain white male bladder cancer mortality rates (1970-1994) in the 508 State Economic Areas (SEA) of the US. Their model consisted of two explanatory variables: population density, a proxy for environmental and behavioral differences in urban/rural life, and lung cancer mortality rates, a proxy for the risk factor smoking, a known risk factor for bladder cancer. These two variables had a global correlation estimate of -0.59 ; however, local correlation estimates were generally more extreme (i.e., more strongly negative; median $=-0.63 ; \mathrm{Q} 3=-0.71$ 
as approximated from their Figure 4), with strongest inverse association in parts of the Northeastern and Midwestern US (their Figure 3). The resulting maps of GWR coefficients for population density and the smoking proxy showed a clear inverse map pattern. When the local smoking proxy parameter was high (primarily in the West and Northeast), the local population density parameter was negative. When the local smoking proxy parameter was negligible, the population density parameter was large and positive (primarily in the Midwest and Southeast). As noted by Wheeler and Tiefelsdorf (2005), the important question is whether this complementary relationship in the parameters is real, meaningful, and interpretable, or an artifact of the statistical method. The natural research question is whether such inverse patterning in regression coefficients is an example of the reversal paradox (Tu et al, 2008) due to strong local correlations between the two variables.

According to the reversal paradox, the association between two variables can be reversed, diminished, or enhanced when another variable is statistically controlled for (Tu et al, 2008). For example, consider two explanatory variables, $x_{1}$ and $x_{2}$, where the bivariate correlation between $x_{1}$ and $y$ is 0.2 , and between $x_{2}$ and $y$ is 0.1 . Figure 4.1 presents the standardized beta coefficients in the multiple regression model $\mu=\beta_{0}+\beta_{1} x_{1}+\beta_{2} x_{2}$. As the correlation between the variables increases, the regression coefficient associated with $x_{1}$ increases and the coefficient associated with $x_{2}$ becomes large and negative - which could lead to a misleading interpretation of the association between $x_{2}$ and $y$. Use of statistical models with correlated data may produce consistent, replicable, yet erroneous results (Tu, et al, 2008). 


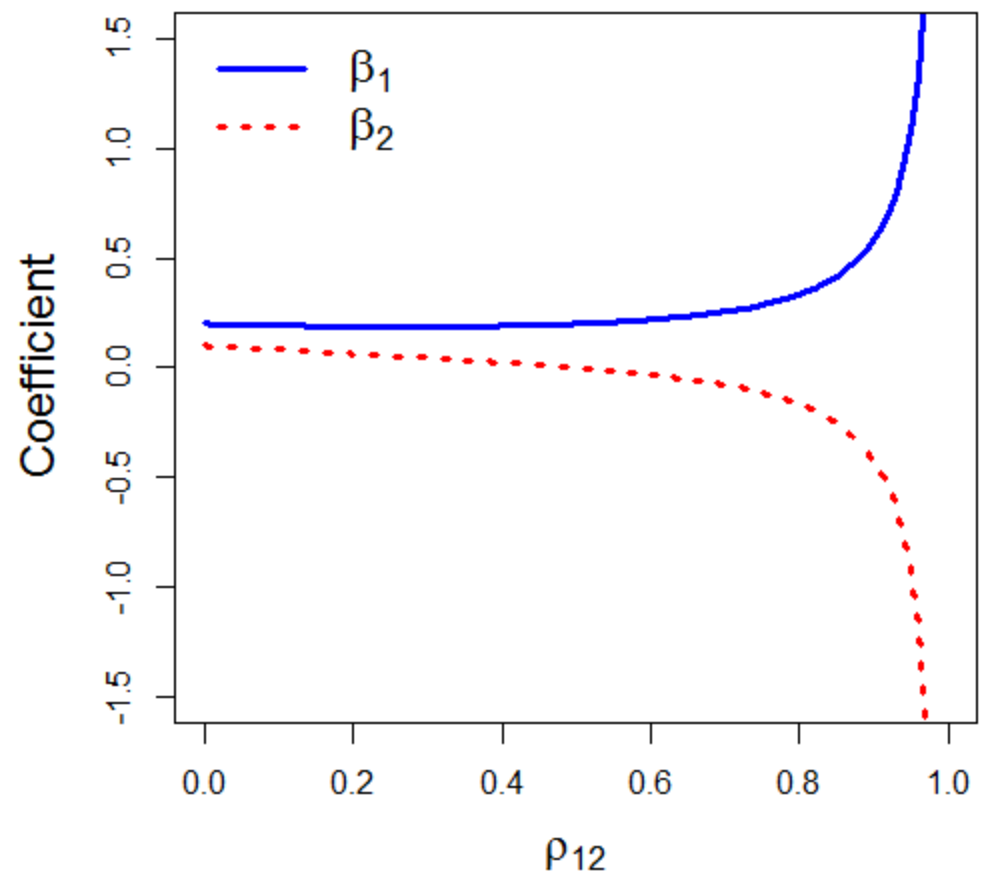

Figure 4.1: Standardized partial regression coefficients in a multiple regression model with two variables.

Note: $\beta_{1}=\left(\rho_{y 1}-\rho_{12} \rho_{y 2}\right) /\left(1-\rho_{12}^{2}\right)$ and $\beta_{2}=\left(\rho_{y 2}-\rho_{12} \rho_{y 1}\right) /\left(1-\rho_{12}^{2}\right)$, where the bivariate correlation between the predictor variables and $y$ are set as $\rho_{y 1}=0.2$ and $\rho_{y 2}=0.1$, respectively.

To address the issue of collinearity with GWR and limit its effects, the geographically weighted lasso (GWL) adds a constraint on the magnitude of the estimated regression coefficients (Wheeler, 2009). GWL also performs local model selection by potentially shrinking some of the estimated regression coefficients to zero in some locations of the study area - thereby, diminishing the adverse effects of the correlation pattern. However, when accurate variable selection is the focus of the analysis, such a strategy makes it difficult to determine whether a variable was excluded from the model due to a lack of association with the outcome or due to its correlation with variables in the model.

Our objective in this paper is to evaluate the impact of collinearity on the geographically weighted regression models of GWR and GWL in a chemical exposure and risk assessment 
context. We use a simulated dataset where the truth is known, and further assess the ability of GWL to control collinearity effects, such as the reversal paradox, when the effects of correlated environmental chemicals are of interest. We begin by describing the process used to simulate data that we propose are environmentally relevant - i.e., regions with low exposure and regions with higher exposures and where different chemicals may have related exposure patterns but not necessarily the same association with a health effect of interest. We conduct GWR and GWL analyses in a scenario with independent chemicals and a scenario with correlated chemicals.

\subsection{Methods}

\subsubsection{Simulating spatially varying exposure and dose-dependent association with an outcome}

Consider the scenario in which there are three predictor variables (e.g., environmental chemicals) that vary over space in a study area (Figure 4.2). We assumed that the first predictor variable, $x_{1}$, was present at high enough levels to be associated with an increase in the mean response in the upper region of the study area, while present only at background levels where there is no increase in mean response in the lower region of the study area. Furthermore, we assumed that

both $x_{2}$ and $x_{3}$ were present at uniform levels across the study area, and that $x_{2}$ was not related to the response variable, while the relationship between $x_{3}$ and the response was moderate.

Additionally, we considered two cases for the relationships among the predictor variables: case 1, where the predictor variables were independent (i.e. multivariate normal with zero correlation); and case 2 , where the predictor variables were correlated (i.e., $\rho_{12}=0.7, \rho_{13}=0.3$ and $\rho_{23}=0$ ). We used a unit grid as the study area, and divided the grid into three equal sized rows. A total of 500 locations were randomly generated inside the study area, and locations falling in the upper $1 / 3$ of the study area were defined as belonging to region $1\left(n_{1}=160\right)$, while 
locations falling in the lower $2 / 3$ of the study area were defined as belonging to region 2 $\left(n_{2}=340\right)$.

For each case, multivariate normal data were simulated separately for region 1 and region 2 . We assumed that the levels of $x_{1}$ were highest in region 1 (mean of 3 ) and negligible in region 2 (mean of 0.1). We further assumed that the mean of both $x_{2}$ and $x_{3}$ was constant (mean of 0.1) across the entire study area. In the case of independence, an identity matrix was used for the covariance, while for the correlated case, the aforementioned correlation pattern was imposed. To simulate the corresponding mean related to the three predictor variables, we used the following nonlinear threshold model:

$$
\mu=\beta_{0}+\beta_{1}\left(x_{1}>\delta\right) x_{1}+\beta_{2} x_{2}+\beta_{3} x_{3}
$$

with parameters defined as $\beta_{0}=\beta_{2}=0, \beta_{1}=2, \beta_{3}=1$, and $\delta=2$. The response variable, $y$, was generated by adding a standard normal error term to the mean. Using this model, we imposed that $x_{1}$ was active in region 1 and inactive in region 2 . More specifically, we allowed $x_{1}$ to be present at high enough levels to be associated with an increase in mean response in region 1, while present only at background levels (i.e., less than the threshold) and not associated with the mean response in region 2 . This specification effectively removed $\beta_{1}$ from the model in region 2 , with $\beta_{1}=0$ for almost all of the locations in region 2 for both the correlated and uncorrelated case. The parameter $\beta_{1}$ was equal to 2 in the majority of locations in region 1 . Hence, there was a simple spatially varying relationship for $x_{1}$ and the outcome variable. Finally, we imposed that $x_{2}$ was not related to the response variable, while the relationship between $x_{3}$ and the response was moderate and uniform across the study area. A total of 100 data sets of size $\mathrm{N}=500$ were 
generated for each case and results are later presented aggregated over the 100 simulated data sets.

\subsubsection{Geographically weighted regression}

In GWR, the spatial coordinates of data are used in the calculation of distances that are input into a kernel function to determine weights for spatial dependence between observations. Local regression models are related through sharing data, but the dependence between regression coefficients at different locations is not specified. For example, consider $n$ observations measured at different locations. The GWR model at location $i$ is:

$$
y_{i}=\mathbf{X}_{i} \boldsymbol{\beta}_{i}+\varepsilon_{i}
$$

where $y_{i}$ is the dependent variable at location $i, \mathbf{X}_{i}$ is the row vector of explanatory variables at location $i, \boldsymbol{\beta}_{i}$ is the column vector of regression coefficients at location $i$, and $\varepsilon_{i}$ is the random error at location $i$. The vector of estimated regression coefficients at location $i$ is

$$
\hat{\boldsymbol{\beta}}_{i}=\left[\mathbf{X}^{T} \mathbf{W}_{i} \mathbf{X}\right]^{-1} \mathbf{X}^{T} \mathbf{W}_{i} \mathbf{y}
$$

where $\mathbf{X}$ is the design matrix of explanatory variables; $\mathbf{W}_{i}$ is the diagonal weights matrix that is calculated for each location $i$ and applies weights to observations $j=1, \ldots, \mathrm{n}$; and $\mathbf{y}$ is the vector of dependent variable values. Examples of kernel functions for defining the weight matrix include the Gaussian function, the bi-square nearest-neighbor function, and the exponential function, used herein. The weight from the exponential kernel function between any location $j$ and the model location $i$ is calculated as

$$
w_{j}(i)=\exp \left(\frac{-d_{i j}}{\phi}\right)
$$


where $d_{i j}$ is the distance between locations $i$ and $j$ and $\phi$ is the kernel bandwidth parameter.

\subsubsection{Geographically weighted lasso}

For a continuous response, the lasso is defined (Tibshirani, 1996) as

$$
\hat{\beta}^{L}=\underset{\beta}{\operatorname{argmin}} \sum_{i=1}^{n}\left(y_{i}-\beta_{0}-\sum_{k=1}^{p} x_{i j} \beta_{k}\right)^{2}+\lambda \sum_{k}\left|\beta_{k}\right|,
$$

where lambda controls the amount of shrinkage of the regression coefficients, the value of which is chosen through algorithms such as LARS (Efron et al, 2004) to find the lowest root mean square prediction error (RMSPE). Wheeler (2009) extended lasso to a geographically weighted version by defining a weighted $\mathbf{X}$ matrix as

$$
\mathbf{X}_{W}=\mathbf{W}_{i}^{1 / 2} \mathbf{X}
$$

and estimating a lasso model with the LARS algorithm corresponding to each of the $i^{\text {th }}$ locations, $i=1, \ldots, \mathrm{n}$.

\subsubsection{Evaluation of models}

The focus of the study was to determine whether the methods were able to correctly detect a strong relationship between $x_{1}$ and the mean response $\left(\beta_{1}=2\right)$ in the upper third of the study grid and a moderate but uniform relationship between $x_{3}$ and the mean response $\left(\beta_{3}=1\right)$ over the entire study area. Additionally, we were also interested in whether or not the methods can correctly discern that there is no relationship between $x_{1}$ and the mean response in the lower $2 / 3$ of the study grid, and no relationship between $x_{2}$ and the mean response over the entire study area. In order to evaluate the performance of GWR and GWL in identifying the spatially varying 
patterns in the coefficients, we started by mapping the average of the coefficient estimates at each location over the study area for both methods.

For each model we calculated the root mean squared error (RMSE) from estimation, the root mean squared prediction error (RMSPE), and the $\mathrm{R}^{2}$ value. The RMSE is defined as

$$
R M S E=\sqrt{\frac{1}{n} \sum_{i=1}^{n}\left[y_{i}-\hat{y}_{i}\right]^{2}},
$$

while RMSPE is defined as

$$
R M S P E=\sqrt{\frac{1}{n} \sum_{i=1}^{n}\left[y_{i}-\hat{y}_{(i)}\right]^{2}},
$$

where $\hat{y}_{(i)}$ is the predicted value of observation $i$ with location $i$ left out of the estimation data set. We then described these summary statistics using the median and IQR over the 100 simulations.

To evaluate the performance of GWL in terms of variable selection, the percentage of coefficient estimates that were positive, negative, or zero were calculated by region for each simulated data set. We summarized the results across the simulated examples using medians and interquartile ranges (IQR). Because GWR does not perform variable selection, we calculated the percentage of coefficient estimates that were positive and negative within each region. Additionally, in an effort to further evaluate the performance of GWR, we approximated the variance of the estimated GWR regression coefficients and created confidence intervals for the estimates at each location based on 1 and 2 standard errors (i.e., $\hat{\beta}_{i j} \pm \operatorname{SE}\left(\hat{\beta}_{i j}\right)$ and $\hat{\beta}_{i j} \pm 2 \operatorname{SE}\left(\hat{\beta}_{i j}\right)$, for the $i=1, \ldots$, $n$ locations, and $j=0, \ldots, k$ parameters). The estimates were then classified as positive, if the confidence interval was above zero, negative if the confidence interval was below zero, and zero 
(negligible) if the confidence interval contained zero. The covariance of the estimated regression coefficients was approximated (Wheeler, 2014) as

$$
\operatorname{Var}\left[\hat{\boldsymbol{\beta}}_{i}\right]=\left[\left(\mathbf{X}^{T} \mathbf{W}_{i} \mathbf{X}\right)^{-1} \mathbf{X}^{T} \mathbf{W}_{i}\right]\left[\left(\mathbf{X}^{T} \mathbf{W}_{i} \mathbf{X}\right)^{-1} \mathbf{X}^{T} \mathbf{W}_{i}\right]^{T} \hat{\sigma}^{2}
$$

where the estimated error variance, $\hat{\sigma}^{2}$, is given as

$$
\hat{\sigma}^{2}=\sum_{i=1}^{n}\left(y_{i}-\hat{y}_{i}\right)^{2} /\left(n-\left(2 \operatorname{trace}(\mathbf{H})-\operatorname{trace}\left(\mathbf{H}^{T} \mathbf{H}\right)\right)\right)
$$

with the $i^{\text {th }}$ row of the hat matrix defined as

$$
\mathbf{H}_{i}=\mathbf{X}_{i}\left(\mathbf{X}^{T} \mathbf{W}_{i} \mathbf{X}\right)^{-1} \mathbf{X}^{T} \mathbf{W}_{i}
$$

\subsection{Results}

The average observed concentration levels across the 100 simulated examples are plotted over the study area for each case in Figure 4.2, where we see that the average levels of $x_{2}$ and $x_{3}$ are uniform over the study area, while the mean level for $x_{1}$ is higher in region 1 (the upper $1 / 3$ of the grid space) as desired. The observed means for both the predictor and response variables are consistent with the study design, and are summarized by case and region in Table 4.1. The observed correlation patterns were also consistent with the study design (results not shown).

Table 4.1: Average predictor and response values across the 100 simulated data sets for the cases of independent chemicals (case 1) and correlated chemicals (case 2).

\begin{tabular}{lrrrr} 
& $\mathrm{X}_{1}$ & $\mathrm{X}_{2}$ & $\mathrm{X}_{3}$ & $\mathrm{Y}$ \\
\hline Case 1 & & & & \\
$\quad$ Region 1 & 3.00 & 0.11 & 0.09 & 5.63 \\
Region 2 & 0.10 & 0.11 & 0.10 & 0.23 \\
Case 2 & & & & \\
$\quad$ Region 1 & 3.00 & 0.11 & 0.11 & 5.62 \\
Region 2 & 0.10 & 0.10 & 0.11 & 0.25 \\
\hline
\end{tabular}



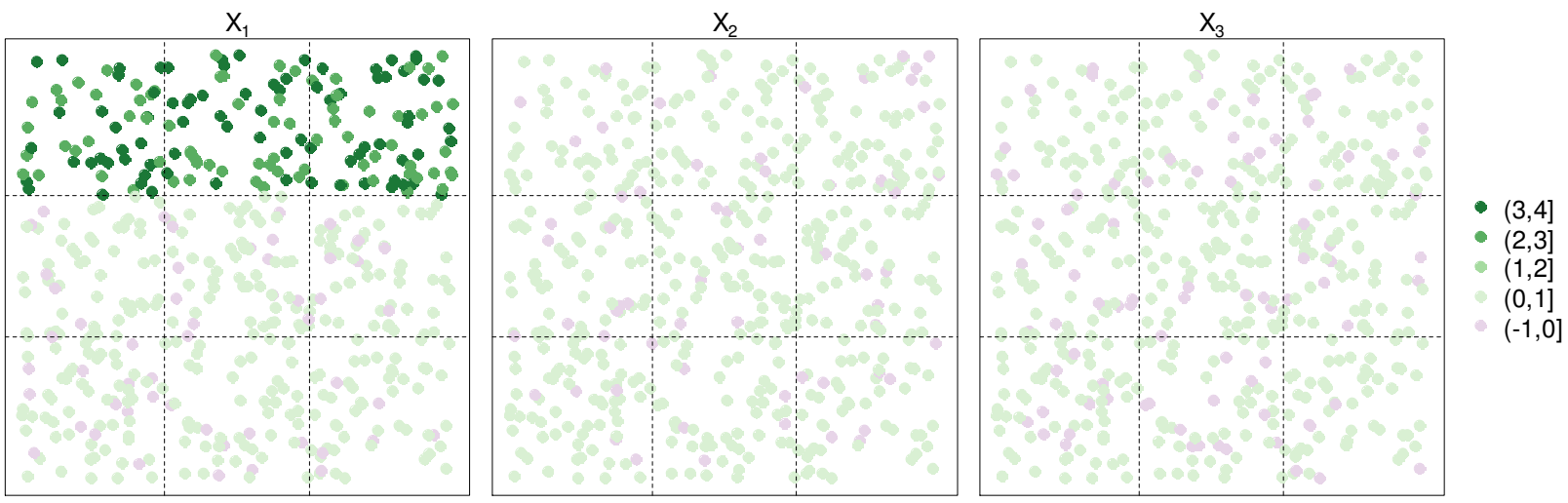

Case 1: Independence
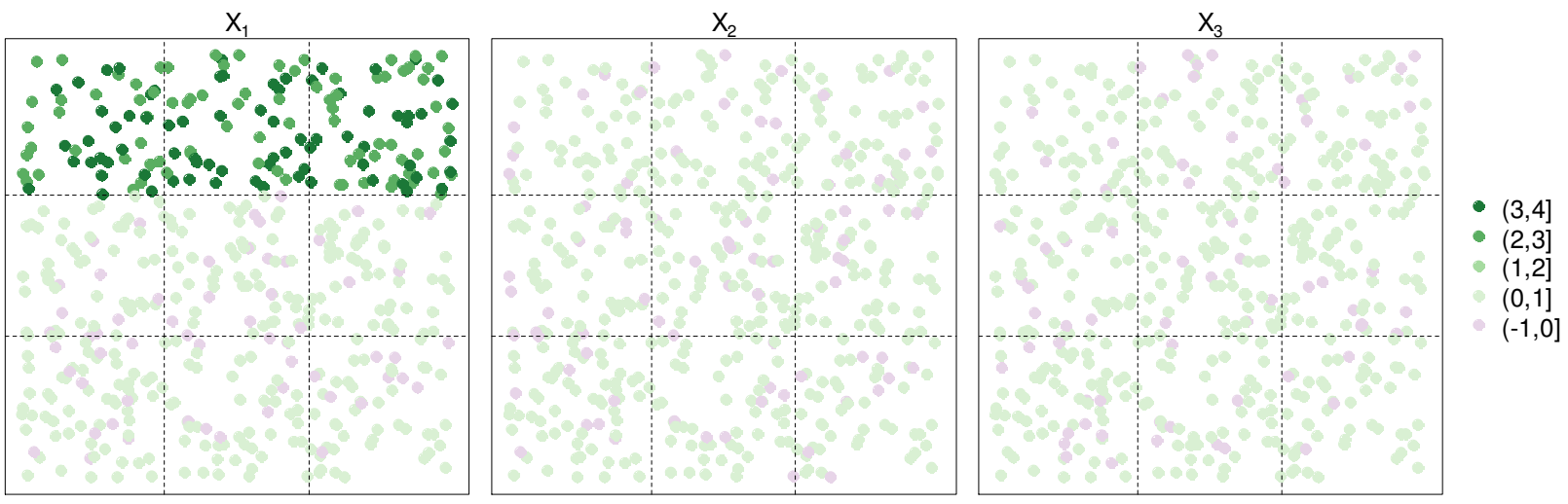

Case 2: Correlation

Figure 4.2: Plots of average simulated concentration values across 100 simulated data sets over a square study area for two scenarios: independent chemicals (case 1) and correlated chemicals (case 2).

The summary statistics across the 100 data sets are listed in Table 4.2. GWL outperformed GWR in terms of RMSPE in the uncorrelated case, while in the correlated case GWL outperformed GWR in terms of both RMSPE and RMSE, with a greater improvement for prediction of the outcome (RMSPE) than for estimation of the outcome (RMSE). 
Table 4.2: Median (IQR) of summary statistics for GWR and GWL models across the 100 simulated data sets for the cases of independent chemicals (case 1) and correlated chemicals (case 2).

\begin{tabular}{ccccccc} 
& \multicolumn{2}{c}{ RMSPE } & \multicolumn{2}{c}{ RMSE } & \multicolumn{2}{c}{$\mathrm{R}^{2}$} \\
\hline Case 1 & & & & & & \\
GWR & 1.4 & $(1.4,1.5)$ & 1.0 & $(1.0,1.2)$ & 0.9 & $(0.9,0.9)$ \\
GWL & 1.2 & $(1.1,1.2)$ & 1.1 & $(0.7,1.2)$ & 0.9 & $(0.9,1.0)$ \\
Case 2 & & & & & & \\
GWR & 1.4 & $(1.4,1.5)$ & 1.2 & $(1.0,1.2)$ & 0.9 & $(0.9,0.9)$ \\
GWL & 1.2 & $(1.1,1.2)$ & 1.1 & $(0.7,1.2)$ & 0.9 & $(0.9,1.0)$ \\
\hline
\end{tabular}

Pairwise plots of the average regression coefficients are shown in Figure 4.3. Correlation in the parameter estimates is evident for both GWR and GWL in the case of independence and in the case of correlated chemicals. In the uncorrelated case, the relationship is most pronounced between the intercept and $\beta_{1}$ parameters (denoted by $\mathrm{b} 0$ and $\mathrm{b} 1$, respectively). In the correlated case, there is a noticeable pattern among all of the parameter estimates, with a strong linear relationship evident between the estimates for $\beta_{2}$ and $\beta_{3}$ (denoted by b2 and b3, respectively). While GWL breaks up some of the strong correlation among the parameter estimates that is evident in GWR, strong relationships are still present between many of the regression coefficients. 

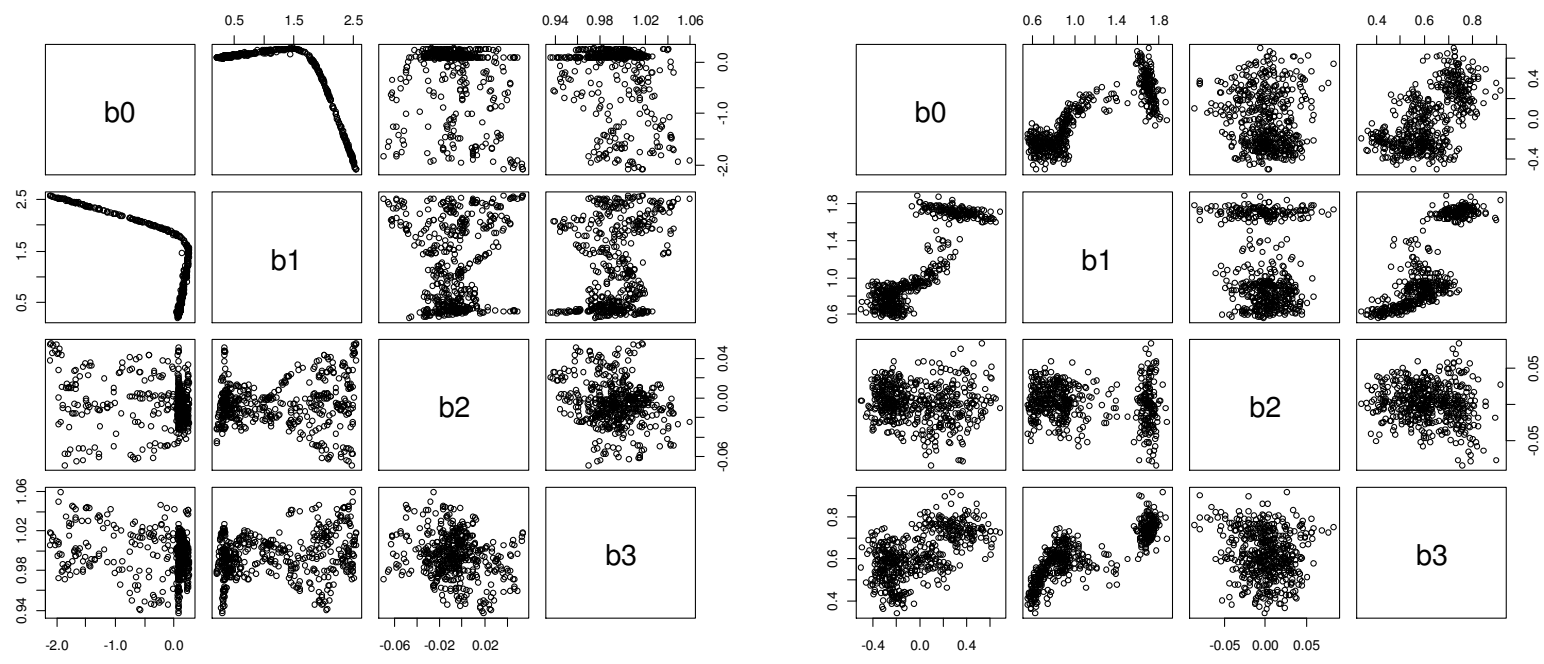

Case 1: Independence (GWR left, GWL right)
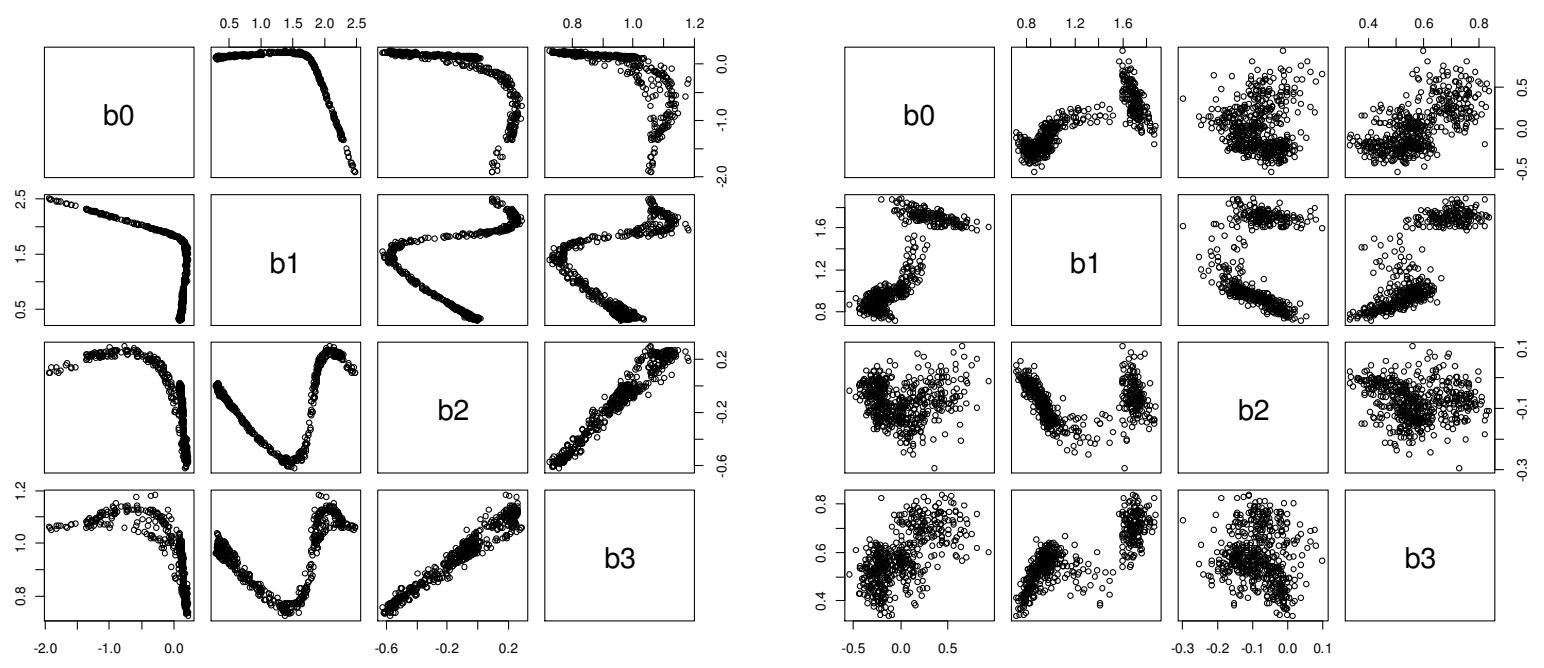

Case 2: Correlation (GWR left, GWL right)

Figure 4.3: Pairwise plots of average regression coefficients across the 100 simulated data sets for the cases of independent chemicals (case 1) and correlated chemicals (case 2) for GWR and GWL.

As demonstrated in the boxplots of the averaged regression coefficients from the models for the 100 simulated datasets (Figure 4.4), GWR appears to accurately capture the importance of $x_{1}$ in region 1 , with distributions centered around 2 for the $\beta_{1}$ estimates in both the independent and correlated case. However, we also see that GWR overstates the importance of $x_{1}$ in region 2, 
with distributions centered above zero for the $\beta_{1}$ estimates regardless of the relationship among the predictor variables. Furthermore, GWL performs shrinkage as expected, demonstrated by the frequent reduction in the magnitude of the parameter estimates when comparing GWR to GWL. However, in both the independent and correlated case, GWL often understates the importance of $x_{1}$ in region 1 and overstates its importance region 2, with distributions for the $\beta_{1}$ estimates centered below 2 in region 1 and around 1 in region 2.

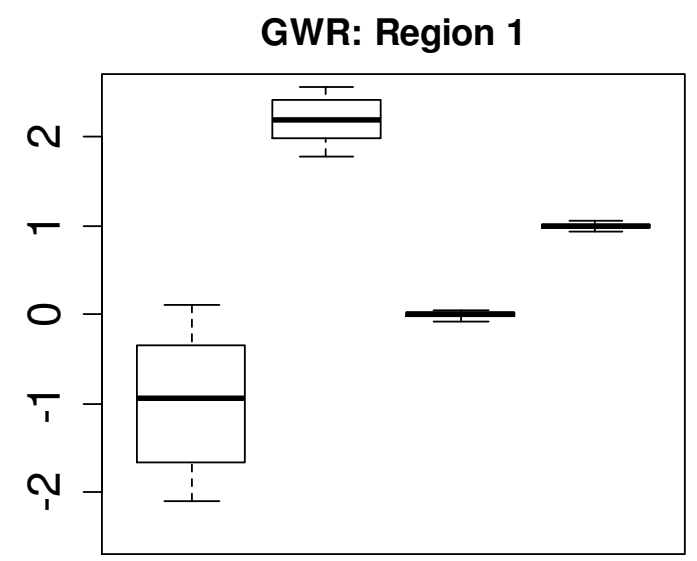

GWR: Region 2

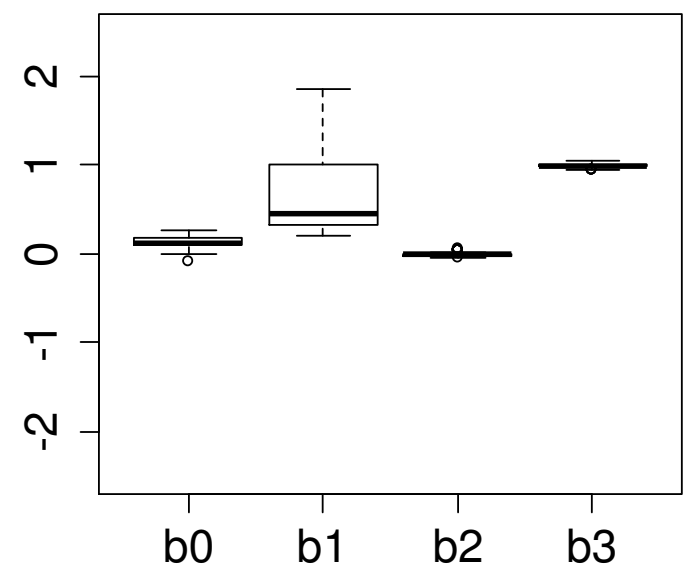

GWL: Region 1

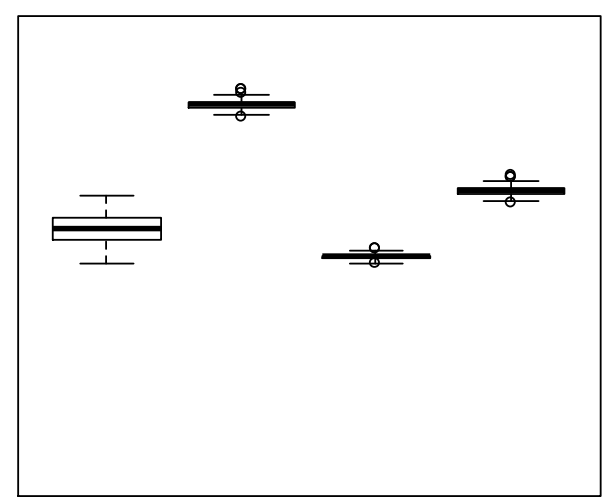

GWL: Region 2

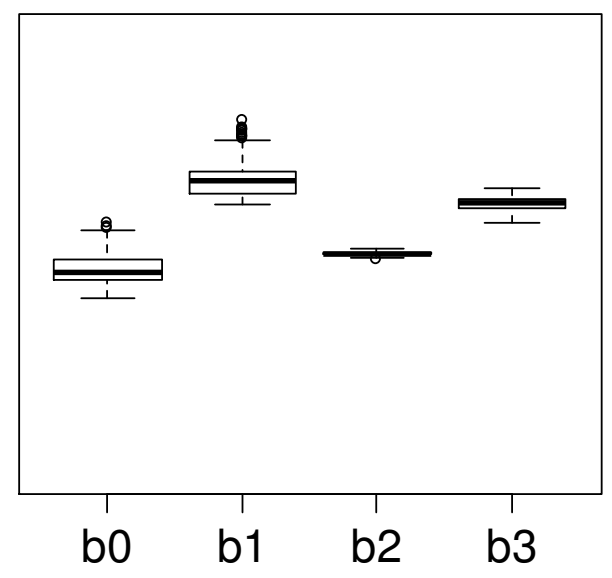

Case 1: Independence (GWR left, GWL right) 


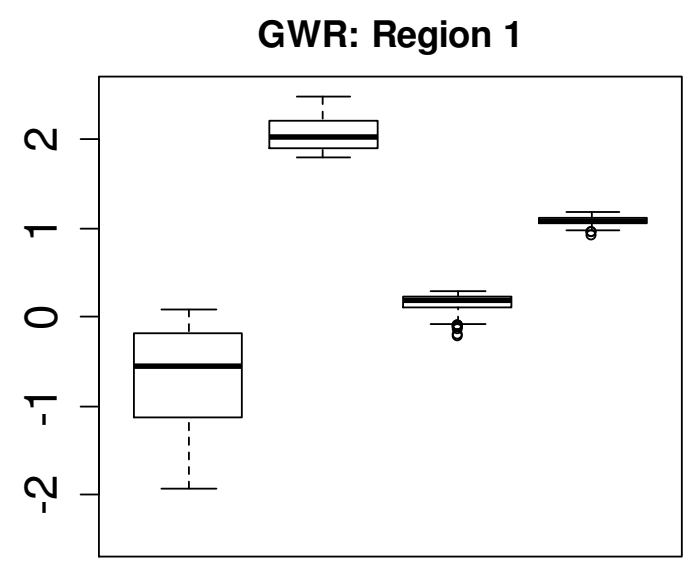

GWR: Region 2

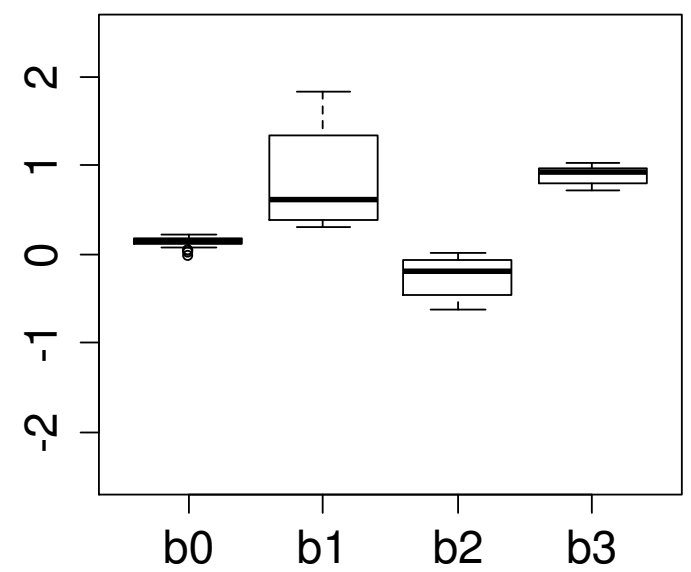

GWL: Region 1

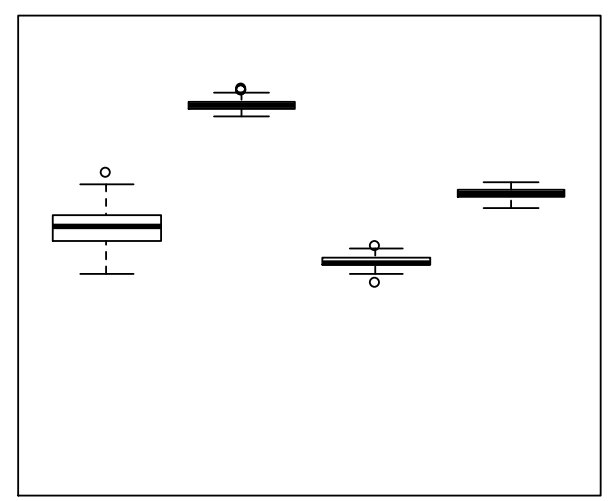

GWL: Region 2

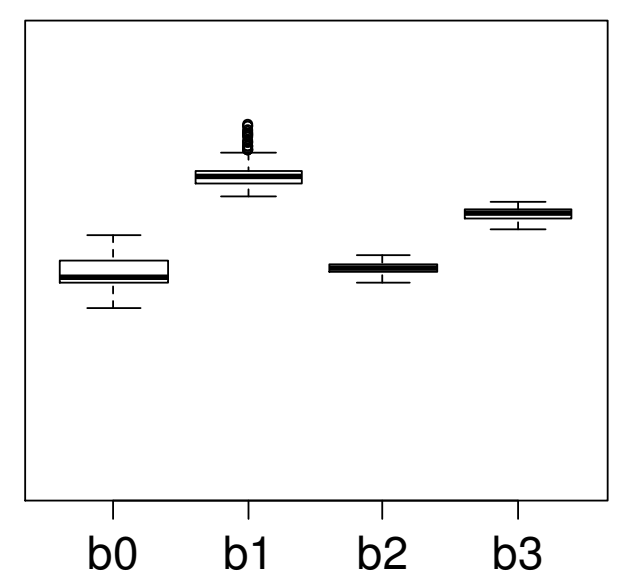

Case 2: Correlation (GWR left, GWL right)

Figure 4.4: Boxplots of average GWR and GWL regression coefficients across 100 simulated datasets for the two study regions for the cases of independent chemicals (case 1) and correlated chemicals (case 2).

The GWR and GWL regression coefficient estimates from the 100 simulated datasets were averaged at each location and plotted in Figure 4.5. The coefficient maps reveal a high degree of correlation between the GWR estimates of $\beta_{0}$ and $\beta_{1}$ in both the independent and correlated cases. This strong negative relationship is also evident in the pairwise scatter plots of the regression coefficients (Figure 4.3). Similarly, correlation in the intercept and $\beta_{1}$ is also apparent in the GWL models, although the correlation between the estimates is not as strong and is largely positive. When examining the coefficient maps for $\beta_{1}$ in both the independent and 
correlated cases, GWR and GWL correctly identified region 1 as the area of highest activity for $x_{1}$ but tended to over-smooth the effect into the upper part of region 2 (i.e., the second row of the grid space). GWL also tended to over-shrink the parameter estimates for $\beta_{1}$ in region 1 , thereby underestimating the effect of $x_{1}$ in the region of activity.
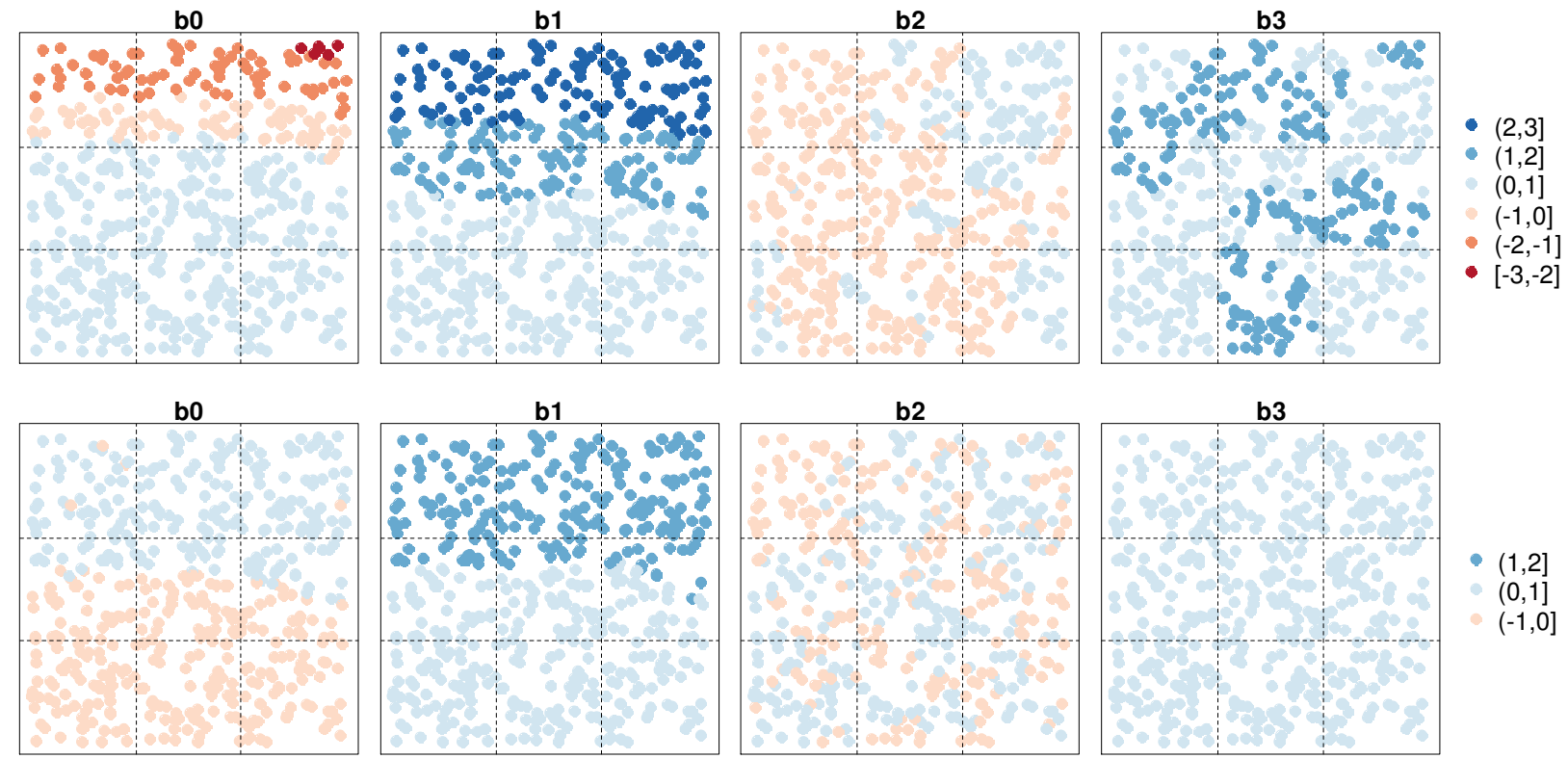

b2

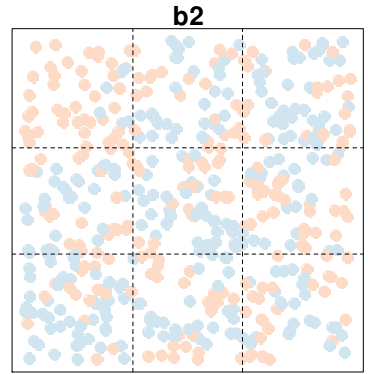

b3

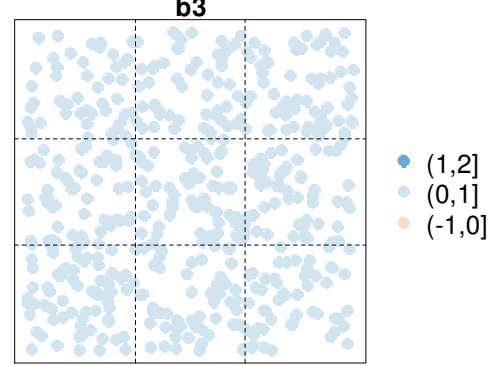

Case 1: Independence (GWR top panel, GWL bottom panel) 

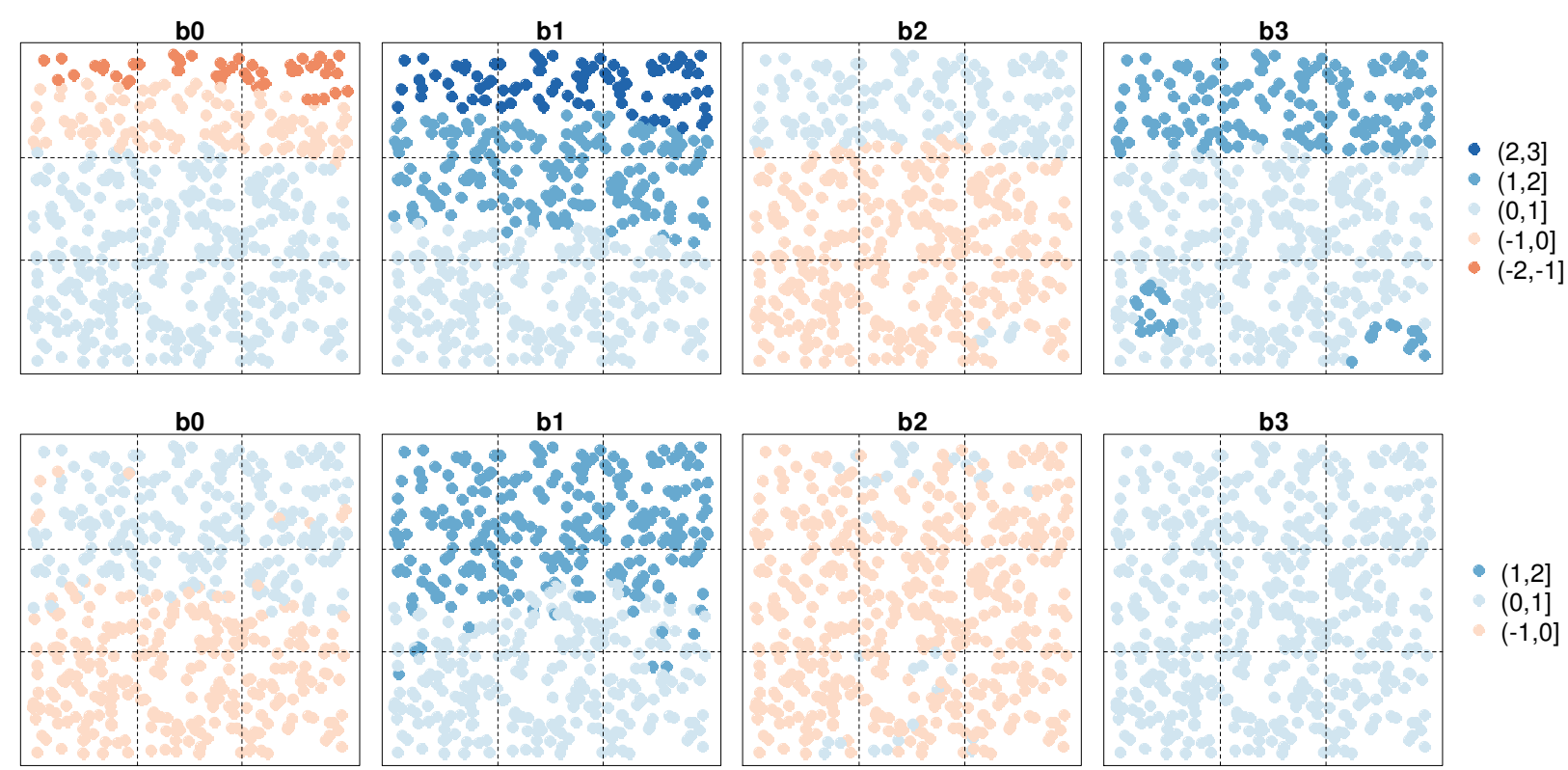

Case 2: Correlation (GWR top panel, GWL bottom panel)

Figure 4.5: Average GWR and GWL regression coefficient estimates over 100 simulated datasets for the cases of independent chemicals (case1) and correlated chemicals (case 2).

When considering the estimated $\beta_{2}$ coefficients, GWR appears to identify several clusters of positive and negative associations in the independent case, while in the case of correlation, it finds a positive association in region 1 and a negative association in region 2, likely a reflection of the high degree of correlation between the predictors $x_{1}$ and $x_{2}$. Although the estimates are small in magnitude, it is clear that GWR is identifying artificial patterns in the $\beta_{2}$ regression coefficients. In contrast, the maps of the GWL estimates for $\beta_{2}$ demonstrate little to no systematic patterning in both the correlated and uncorrelated case, suggesting that GWL is able to break up some the artificial patterning seen in the GWR estimates $\beta_{2}$.

Finally, with respect to $\beta_{3}$, GWR appears to incorrectly identify several clusters of a stronger positive relationship between $x_{3}$ and the response variable in the uncorrelated case. Furthermore, in the correlated case, GWR incorrectly identifies a strong spatial pattern in the $\beta_{3}$ estimates, 
with region 1 appearing to be an area of high activity. The similarity in the GWR coefficient maps of $\beta_{2}$ and $\beta_{3}$ (Figure 4.5) reflects the strong linear positive relationship demonstrated in the pairwise plots of the estimated GWR regression coefficients in the correlated case (Figure 4.3). The artificial spatial pattern in the GWR estimates of $\beta_{3}$ parallels the true spatial variation in $\beta_{1}$, and is likely induced by the correlation among $x_{1}$ and $x_{3}$. In contrast, GWL is able to reduce the correlation between the $\beta_{2}$ and $\beta_{3}$ estimates, and appears to correctly identify the uniform moderate relationship between $x_{3}$ and the response regardless of the relationship among the predictor variables.

The percentage of positive and negative GWR coefficient estimates are summarized by region for each correlation case in the upper half of Table 4.3. We see that across the simulated data sets, the GWR estimates of $\beta_{1}$ were positive nearly 100 percent of the time in region 1 and 2 for both the independent and correlated cases. This is further evidence that GWR overstates the importance of $\beta_{1}$ in region 2 . When considering $\beta_{2}, 53 \%$ of the GWR estimates in regions 1 and 2 were negative in the case of independence for at least half of the simulated data sets, while $28 \%$ and $77 \%$ of the GWR estimates in regions 1 and 2, respectively, were negative in the case of correlation. Given that $x_{2}$ has no relationship with the outcome in the simulated data, we suspect the presence of the reversal paradox, which could lead to incorrect inference about the impact of this predictor.

Similarly, as shown in the lower half of Table 4.3, the GWL estimates of $\beta_{1}$ were positive nearly 100 percent of the time in region 1 and 2, for both the independent and correlated cases. This indicates that GWL failed to appropriately perform variable selection for $x_{1}$ in region 2, the 
region of inactivity. Furthermore, in at least half of the simulated examples, $17 \%$ and $35 \%$ of the GWL estimates of $\beta_{3}$ in regions 1 and 2 , respectively, were zero in the case of independence, and $15 \%$ and $35 \%$ of the estimates of $\beta_{3}$ in regions 1 and 2 , respectively, were zero in the case of correlation. Given that $x_{3}$ is moderately positively associated with the outcome across the study area, these results could lead to the incorrect conclusion that this predictor is not positively associated with the adverse outcome.

Table 4.3: Median (IQR) percentage of GWR and GWL coefficient estimates that were positive, negative, and zero across the 100 simulated data sets for the cases of independent chemicals (case 1) and correlated chemicals (case 2).

\begin{tabular}{|c|c|c|c|c|c|c|c|c|}
\hline \multicolumn{9}{|l|}{ GWR } \\
\hline Case 1 & \multicolumn{2}{|r|}{ b0 } & \multicolumn{2}{|r|}{ b1 } & \multicolumn{2}{|r|}{$\mathrm{b} 2$} & \multicolumn{2}{|r|}{ b3 } \\
\hline \multicolumn{9}{|l|}{ Region 1} \\
\hline Positive & 12 & $(6,21)$ & 100 & $(100,100)$ & 47 & $(39,64)$ & 100 & $(100,100)$ \\
\hline Negative & 88 & $(79,94)$ & 0 & $(0,0)$ & 53 & $(36,61)$ & 0 & $(0,0)$ \\
\hline Zero & -- & -- & -- & - & -- & - & -- & - \\
\hline \multicolumn{9}{|l|}{ Region 2} \\
\hline Positive & 76 & $(66,86)$ & 96 & $(92,99)$ & 47 & $(40,61)$ & 100 & $(100,100)$ \\
\hline Negative & 24 & $(14,34)$ & 4 & $(1,8)$ & 53 & $(39,60)$ & 0 & $(0,0)$ \\
\hline Zero & -- & -- & -- & -- & -- & -- & -- & -- \\
\hline \multicolumn{9}{|l|}{ Case 2} \\
\hline \multicolumn{9}{|l|}{ Region 1} \\
\hline Positive & 15 & $(8,25)$ & 100 & $(100,100)$ & 73 & $(64,83)$ & 100 & $(100,100)$ \\
\hline Negative & 85 & $(75,93)$ & 0 & $(0,0)$ & 28 & $(18,36)$ & 0 & $(0,0)$ \\
\hline Zero & -- & - & -- & - & -- & - & -- & - \\
\hline \multicolumn{9}{|l|}{ Region 2} \\
\hline Positive & 78 & $(69,89)$ & 97 & $(92,100)$ & 23 & $(13,31)$ & 100 & $(100,100)$ \\
\hline Negative & 22 & $(11,31)$ & 3 & $(0,8)$ & 77 & $(69,87)$ & 0 & $(0,0)$ \\
\hline Zero & -- & -- & -- & -- & -- & -- & -- & -- \\
\hline \multicolumn{9}{|l|}{ GWL } \\
\hline Case 1 & & b0 & & b1 & & b2 & & b3 \\
\hline \multicolumn{9}{|l|}{ Region 1} \\
\hline Positive & 62 & $(16,68)$ & 100 & $(100,100)$ & 27 & $(0,36)$ & 83 & $(72,86)$ \\
\hline Negative & 0 & $(0,35)$ & 0 & $(0,0)$ & 27 & $(4,38)$ & 0 & $(0,1)$ \\
\hline Zero & 38 & $(33,49)$ & 0 & $(0,0)$ & 55 & $(44,63)$ & 17 & $(14,26)$ \\
\hline \multicolumn{9}{|l|}{ Region 2} \\
\hline Positive & 7 & $(5,27)$ & 100 & $(47,100)$ & 15 & $(0,23)$ & 65 & $(61,81)$ \\
\hline Negative & 19 & $(18,21)$ & 0 & $(0,12)$ & 16 & $(9,21)$ & 0 & $(0,0)$ \\
\hline
\end{tabular}




\begin{tabular}{lrrrrrrrr}
$\quad$ Zero & 74 & $(51,76)$ & 0 & $(0,37)$ & 81 & $(52,85)$ & 35 & $(18,39)$ \\
Case 2 & & & & & & & & \\
Region 1 & & & & & & & & \\
$\quad$ Positive & 57 & $(17,66)$ & 100 & $(100,100)$ & 0 & $(0,36)$ & 85 & $(75,88)$ \\
$\quad$ Negative & 1 & $(0,30)$ & 0 & $(0,0)$ & 42 & $(26,53)$ & 0 & $(0,1)$ \\
$\quad$ Zero & 42 & $(34,51)$ & 0 & $(0,0)$ & 44 & $(37,55)$ & 15 & $(13,23)$ \\
Region 2 & & & & & & & & \\
$\quad$ Positive & 8 & $(5,26)$ & 100 & $(46,100)$ & 0 & $(0,20)$ & 65 & $(63,82)$ \\
$\quad$ Negative & 19 & $(17,22)$ & 0 & $(0,12)$ & 28 & $(26,31)$ & 0 & $(0,1)$ \\
$\quad$ Zero & 74 & $(50,76)$ & 0 & $(0,40)$ & 68 & $(54,72)$ & 35 & $(16,37)$ \\
\hline
\end{tabular}

On average, the true $\beta_{1}$ parameter was nonzero (i.e., $\beta_{1}=2$ ) at $84 \%$ of locations in region 1 and $3 \%$ of locations in region 2 for both case 1 and case 2 .

The results of applying 1 and 2 standard errors to the GWR estimated coefficients to classify them as positive, negative, or zero are listed in Table 4.4. Using the 1-SE criteria, GWR incorrectly classified $83 \%$ of $\beta_{1}$ estimates in region 2 as positive at least half of the time for the independent case, and incorrectly classified $84 \%$ of $\beta_{1}$ estimates in region 2 as positive at least half of the time when the predictors were correlated. Similarly, when applying the 2-SE criteria, GWR incorrectly classified $64 \%$ of $\beta_{1}$ estimates in region 2 as positive at least half of the time for the independent case, and incorrectly classified $66 \%$ of $\beta_{1}$ estimates in region 2 as positive at least half of the time in the correlated case. This implies that GWR frequently yields nonnegligible positive estimates of $\beta_{1}$ in the region of inactivity.

Furthermore, when applying the 1-SE rule, we see that in the case of independence GWR correctly classified $64 \%$ and $72 \%$ of the $\beta_{2}$ estimates as zero in the upper and lower regions, respectively, at least half of the time. In the case of correlated predictors, only $51 \%$ and $44 \%$ of the $\beta_{2}$ estimates were correctly classified at least half of the time in the upper and lower regions respectively. Finally when using the $2-\mathrm{SE}$ criteria, $29 \%$ of the $\beta_{2}$ estimates in region 1 were incorrectly classified as positive at least half of the time when the predictors were correlated. 
Thus, even when allowing "small” estimates to be considered as negligible, GWR results can still lead to erroneous inference about the nature of a predictor variable that is not associated with the response.

Table 4.4: Median (IQR) percentage of GWR coefficient estimates that were positive, negative, and zero across the 100 simulated data sets when considering \pm 1 and \pm 2 standard errors of regression coefficient estimates for the cases of independent chemicals (case 1) and correlated chemicals (case 2).

\section{GWR (1 SE)}

\begin{tabular}{lrrrrrrrr}
\hline Case 1 & \multicolumn{1}{c}{$\mathrm{b} 0$} & \multicolumn{2}{c}{$\mathrm{b} 1$} & & $\mathrm{~b} 2$ & \multicolumn{2}{c}{$\mathrm{b} 3$} \\
\cline { 2 - 8 } Region 1 & & & & & & & & \\
$\quad$ Positive & 4 & $(1,10)$ & 100 & $(100,100)$ & 16 & $(6,27)$ & 100 & $(99,100)$ \\
$\quad$ Negative & 73 & $(64,82)$ & 0 & $(0,0)$ & 15 & $(8,28)$ & 0 & $(0,0)$ \\
$\quad$ Zero & 21 & $(16,28)$ & 0 & $(0,0)$ & 64 & $(54,73)$ & 0 & $(0,1)$ \\
Region 2 & & & & & & & & \\
$\quad$ Positive & 35 & $(22,44)$ & 83 & $(73,92)$ & 12 & $(7,18)$ & 100 & $(100,100)$ \\
$\quad$ Negative & 3 & $(1,6)$ & 0 & $(0,1)$ & 14 & $(7,22)$ & 0 & $(0,0)$ \\
$\quad$ Zero & 60 & $(53,70)$ & 17 & $(8,24)$ & 72 & $(65,77)$ & 0 & $(0,0)$
\end{tabular}

Case 2

Region 1

$\begin{array}{lllllllll}\text { Positive } & 6 & (1,10) & 100 & (100,100) & 39 & (30,53) & 100 & (100,100)\end{array}$

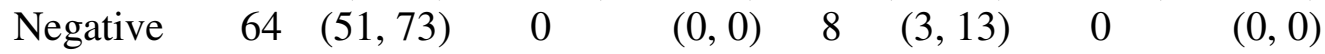

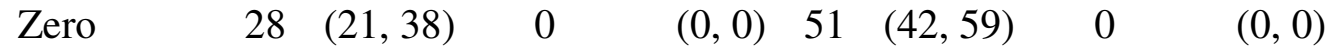

Region 2

Positive $\quad 39 \quad(27,50) \quad 84 \quad(69,92) \quad 3 \quad(0,9) \quad 100 \quad(100,100)$

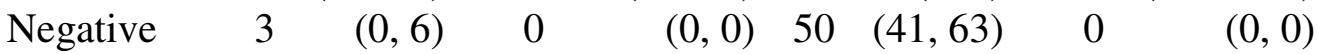

$\begin{array}{lllllllll}\text { Zero } & 58 & (48,68) & 16 & (8,29) & 44 & (35,53) & 0 & (0,0)\end{array}$

GWR (2 SE)

\begin{tabular}{lrrrrrrrr}
\hline Case 1 & \multicolumn{1}{c}{$\mathrm{b} 0$} & \multicolumn{2}{c}{$\mathrm{b} 1$} & \multicolumn{2}{c}{$\mathrm{b} 2$} & \multicolumn{1}{c}{$\mathrm{b} 3$} \\
\cline { 2 - 8 } Region 1 & & & & & & & & \\
$\quad$ Positive & 0 & $(0,3)$ & 100 & $(100,100)$ & 1 & $(0,6)$ & 98 & $(96,100)$ \\
$\quad$ Negative & 53 & $(44,63)$ & 0 & $(0,0)$ & 2 & $(0,6)$ & 0 & $(0,0)$ \\
$\quad$ Zero & 45 & $(36,54)$ & 0 & $(0,0)$ & 94 & $(88,98)$ & 2 & $(0,4)$ \\
Region 2 & & & & & & & & \\
$\quad$ Positive & 8 & $(4,14)$ & 64 & $(54,78)$ & 1 & $(0,3)$ & 99 & $(98,100)$ \\
$\quad$ Negative & 0 & $(0,0)$ & 0 & $(0,0)$ & 1 & $(0,3)$ & 0 & $(0,0)$ \\
$\quad$ Zero & 91 & $(86,96)$ & 36 & $(23,46)$ & 96 & $(94,99)$ & 1 & $(0,2)$ \\
Case 2 & & & & & & & & \\
Region 1 & & & & & & & & \\
$\quad$ Positive & 0 & $(0,4)$ & 100 & $(100,100)$ & 12 & $(5,20)$ & 100 & $(98,100)$ \\
$\quad$ Negative & 33 & $(23,45)$ & 0 & $(0,0)$ & 1 & $(0,3)$ & 0 & $(0,0)$ \\
$\quad$ Zero & 64 & $(53,72)$ & 0 & $(0,0)$ & 86 & $(79,93)$ & 0 & $(0,3)$
\end{tabular}




\begin{tabular}{lrrrrrrrr} 
Region 2 & & & & & & & \\
Positive & 8 & $(3,16)$ & 66 & $(49,76)$ & 0 & $(0,0)$ & 99 & $(97,100)$ \\
Negative & 0 & $(0,0)$ & 0 & $(0,0)$ & 31 & $(20,42)$ & 0 & $(0,0)$ \\
Zero & 91 & $(84,96)$ & 34 & $(24,50)$ & 69 & $(57,79)$ & 1 & $(0,3)$ \\
\hline
\end{tabular}

On average, the true $\beta_{1}$ parameter was nonzero (i.e., $\beta_{1}=2$ ) at $84 \%$ of locations in region 1 and $3 \%$ of locations in region 2 for both case 1 and case 2 . A parameter estimate was counted as zero if its confidence interval based on one or two standard errors contained zero.

As an illustrative example, we randomly chose one simulated data set for each correlation case and plotted the corresponding estimated regression coefficients from GWR and GWL, using open circles for the negligible estimates (i.e., GWR estimates with confidence intervals containing zero or GWL estimates of zero) (Figure 4.6). This example visually supports the aggregate results given in Table 4.3 and 4.4. GWR accurately identified region 1 as the region of high activity for $x_{1}$, but overstated the effect of $x_{1}$ in region 2 , where the predictor is inactive. In addition, in the correlated case, GWR produced a cluster of non-negligible positive estimates for $\beta_{2}$ in region 1 , and non-negligible negative estimates for $\beta_{2}$ in region 2 . Finally, while GWL was able to correctly perform variable selection for $x_{2}$ with some frequency (i.e., estimate that $\beta_{2}$ was zero as shown by the open circles), we see that GWL was again unable to correctly identify the spatially varying pattern of $\beta_{1}$ in the case of independence and in the case of correlation. More specifically, GWL estimated a nearly uniform effect for $\beta_{1}$ across the study area, understating the effect of $x_{1}$ in region 1, and failing to perform appropriate variable selection for $x_{1}$ in region 2 (implying that $x_{1}$ is active in region 2 ). 

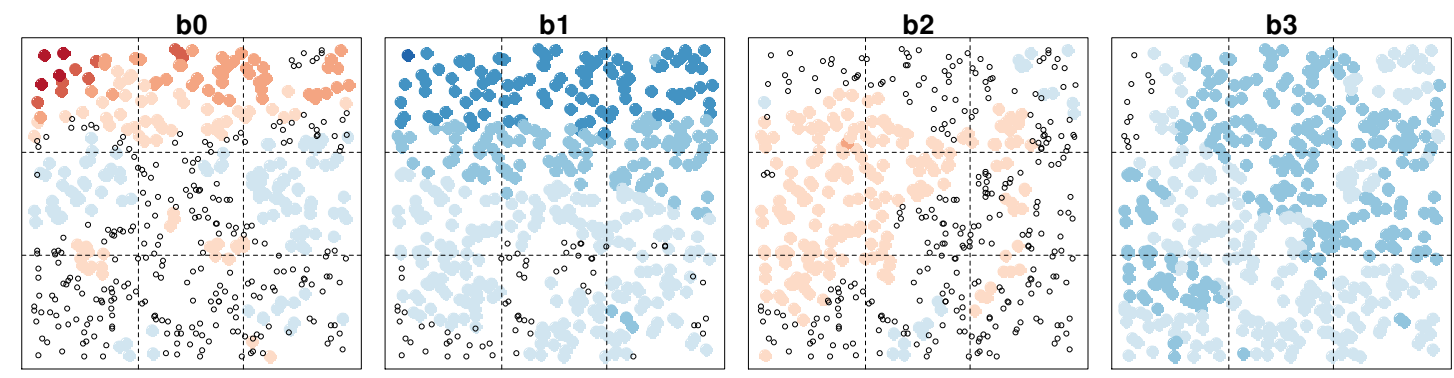

- $(3,4]$

$(2,3]$

- $(1,2]$

- $(0,1]$

$(-1,0]$

- $(-2,-1]$

- $(-3,-2]$

- $[-4,-3]$
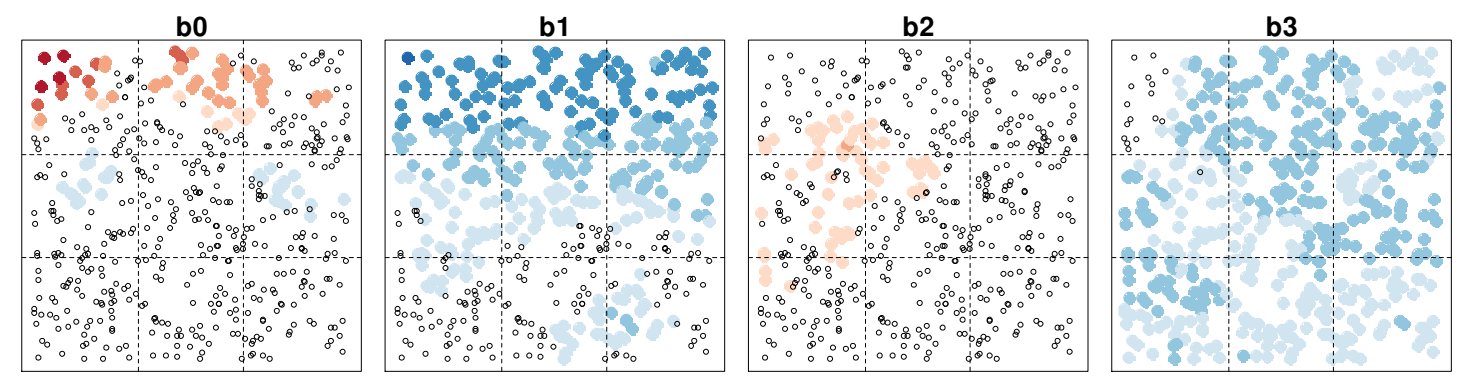

- $(3,4]$

- $(2,3]$

- $(1,2]$

- $(0,1]$

- $(-1,0]$

- $(-2,-1]$

- $(-3,-2]$

- $[-4,-3]$
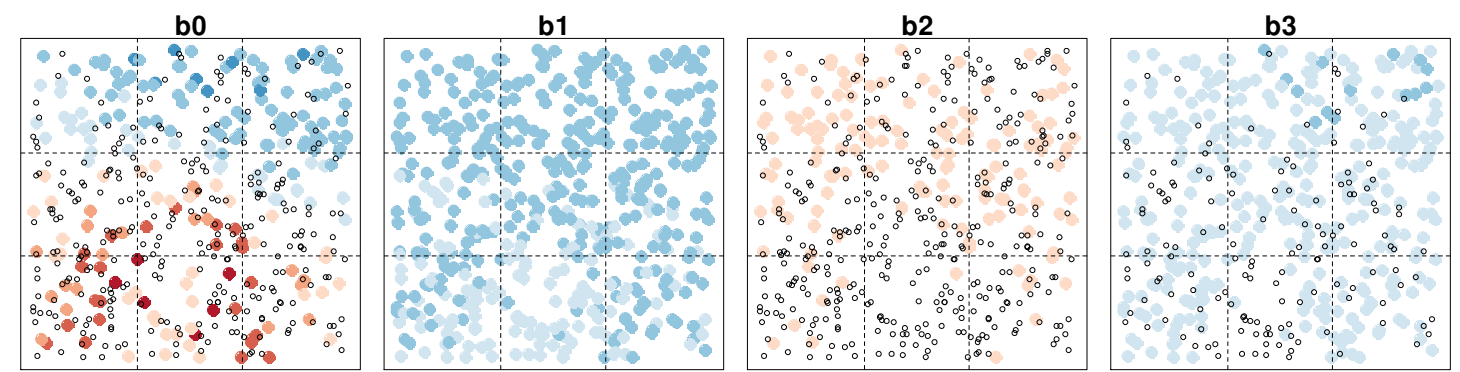

- $(3,4]$

- $(2,3]$

- $(1,2]$

- $(0,1]$

$(-1,0]$

- $(-2,-1]$

- $(-3,-2]$

- $[-4,-3]$

Case 1: Independence: GWR 1-SE (top panel), GWR 2-SE (middle panel), GWL (bottom panel) 

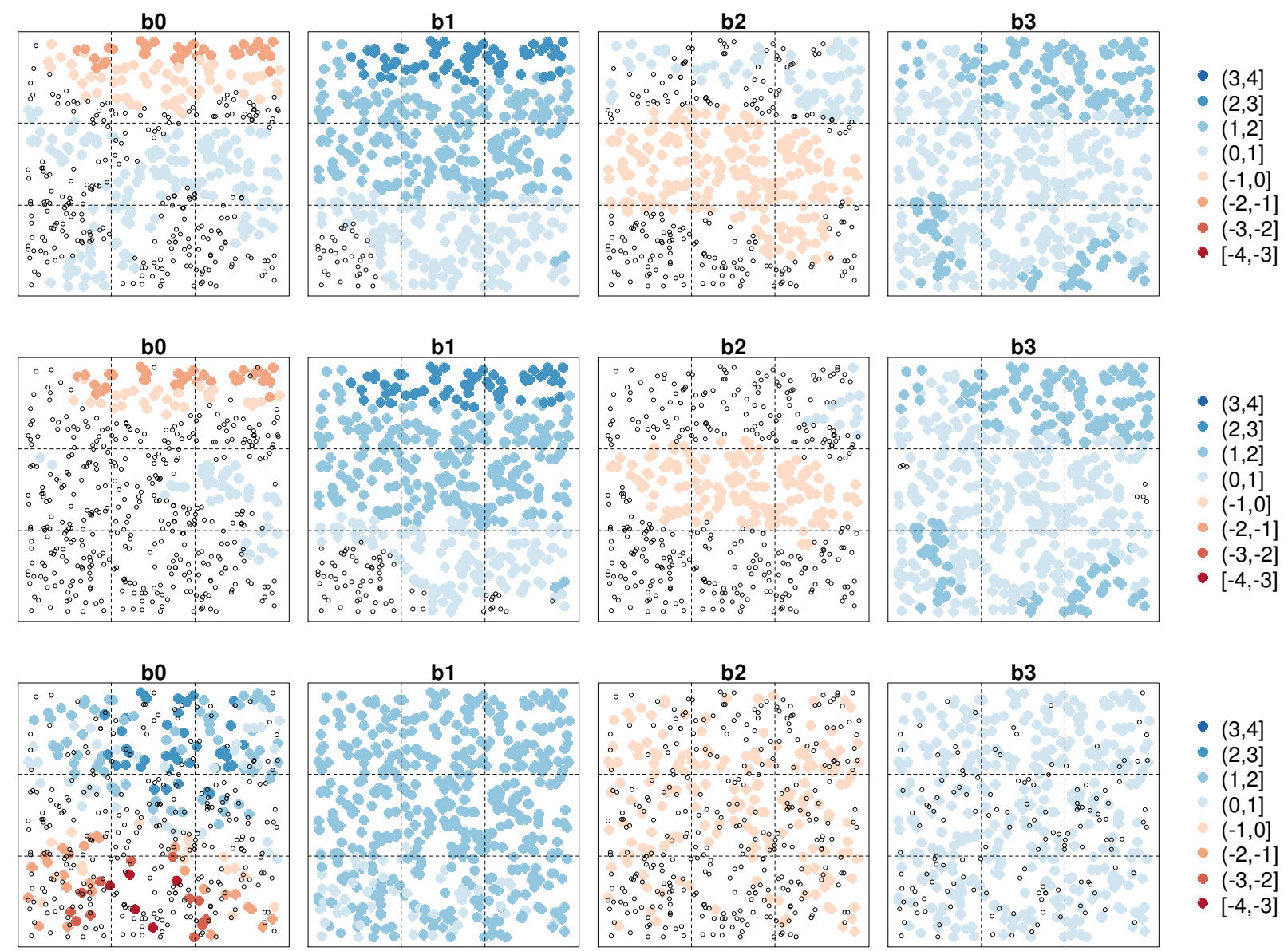

- $(3,4]$

- $(2,3]$

- $(1,2]$

- $(0,1]$

- $(-1,0]$

- $(-2,-1]$

- $(-3,-2]$

- $[-4,-3]$

Case 2: Correlation: GWR 1-SE (top panel), GWR 2-SE (middle panel), GWL (bottom panel)

Figure 4.6: Estimated regression coefficients from GWR and GWL for one simulation of data under the cases of independent chemicals (case 1) and correlated chemicals (case 2).

\subsection{Discussion and conclusions}

We have evaluated the ability of the geographically weighted regression methods of GWR and GWL to detect signal from noise in the context of modeling the associations of environmental chemicals and an adverse health effect using a simulation study with both independent and correlated chemicals. We found that GWR was able to identify regions of high activity for an important chemical when the predictors were independent and when they were highly correlated, but it demonstrated a tendency to overstate the importance of this chemical in its region of inactivity. Furthermore, GWR suffered from the reversal paradox for less important chemicals 
when the chemicals were correlated, as the variable that was not associated with the outcome was largely positive in the upper study region and largely negative in the lower study region. We also found that with GWL the signal of the most important chemical was diminished, with less distinction between the inactive and active study regions, regardless of the correlation among the chemicals.

Previous work has addressed the issue of collinearity in GWR. Wheeler and Tiefelsdorf (2005) first demonstrated the link between collinearity in GWR and correlation of estimated regression coefficients using simulation studies. These authors introduced systematic collinearity into the model by adding correlation to a pair of covariates and found consistent evidence of increasing correlation in GWR coefficients with increasing collinearity. Wheeler and Calder (2007) used two simulation studies to evaluate the coverage probability and accuracy of the regression coefficients from GWR. Results of the simulation studies include low coverage probabilities for the GWR coefficients and consistently increasing error in the coefficients when collinearity is increased. Wheeler (2007) conducted a simple experiment by systematically increasing collinearity in a dataset to demonstrate that a penalized form of GWR, geographically weighted ridge regression, reduces the extreme effects of collinearity that afflict GWR. More recent simulation study work confirms that a non-negligible amount of spatial variation of and correlation between GWR coefficient surfaces is inherently generated by the method (Páez, Farber, and Wheeler, 2011). This work finds that the false positive rates for GWR coefficients are typically much higher than convention would mandate, from less than $10 \%$ to more than $50 \%$ of the time (depending on the true correlation level between two covariates) when the true underlying process is stationary. 
Wheeler (2009) expanded the simulation study of Wheeler and Calder (2007) to have four explanatory variables and 196 observations in a study of the performance of GWR and GWL. This work compared the coefficient accuracy and the predictive performance of the models in the presence of collinearity. In these experiments, 100 realizations of a data generating process were used with the true local coefficients sampled from a multivariate normal distribution. These simulation studies show that the performance of GWR in both prediction and coefficient accuracy can be improved by constraining the magnitude of its regression coefficients with techniques designed to remediate collinearity. However, the experiments reported in the paper show that the correlation between local coefficients is reduced but not eliminated with GWL, and that although GWL can shrink some coefficients to zero to stabilize the model, the estimates still tend to be positively correlated with those from GWR (Páez, Farber, and Wheeler, 2011).

We have extended these results in the case of three environmental chemicals to identify evidence of the reversal paradox and evaluate the correct identification of local 'hot spots' or regions of high activity for one chemical. Our results demonstrate that while GWR can correctly identify a region of high activity for one chemical, it had difficulty identifying regions of inactivity or low exposure. Additionally, GWR artificially induces spatial patterning, and suffers from the reversal paradox in the setting of highly correlated predictor variables. Finally, we have shown that while GWL reduces the correlation among the coefficient estimates and tempers the reversal paradox that is problematic with GWR, it suffers from an inability to adequately distinguish local regions of high activity regardless of the relationship among the predictor variables. The implications of our findings for environmental risk analysis is that GWR may incorrectly identify some chemicals as positively or negatively associated with disease risk, and GWL may not correctly estimate the magnitude of association for an important chemical in some regions of the study 
area. Given these findings, more methodological development is required to better estimate effects of correlated environmental chemicals for diseases with environmental factors, such as many cancers. 


\title{
Chapter 5
}

\author{
Local Weighted Quantile Sum Regression
}

\subsection{Introduction}

In this chapter, we extend the WQS method to accommodate spatially varying effects by developing local weighted quantile sum regression (LWQS), a spatially weighted version of WQS, with the inclusion of a variable subspace strategy. We briefly revisit WQS regression (discussed at length in Chapters 2 and 3) and then extend the method to accommodate spatially varying effects in a detailed discussion of LWQS. Finally, we present a simulation study that assesses the performance of LWQS in a variety of environmentally relevant scenarios.

\subsection{Review}

Recall that WQS regression estimates a weighted linear index of exposures (e.g., environmental chemicals) in which the individual component weights are empirically determined. Simultaneous estimation of the exposure weights and an overall WQS index effect allows us to make generalized inference regarding exposure to the mixture and identify the individual components most strongly related to the outcome.

The WQS approach considers data with $c$ components scored into quantiles that are reasonable (e.g., all environmental chemical exposures) to combine into an index and potentially have a common outcome. The association between exposure and disease risk is constrained to be in the 
same direction for all components included in the index. The weights are constrained be between 0 and 1 and sum to 1 , reducing dimensionality and mitigating issues associated with collinearity. Further, scoring the exposure concentrations into quantiles reduces the influence of outliers in skewed distributions. By convention, concentrations are often scored into quartiles, and quartiles will be used herein. We note however, that the method of data pre-processing (e.g., choice of quantiles) is still an open area of research.

Traditionally, WQS incorporates a bootstrap step in the calculation of the empirical weights, which has been shown to improve accuracy in variable selection (Carrico et al. 2014). WQS regression maximizes the likelihood for the nonlinear model

$$
g(\mu)=\beta_{0}+\beta_{1}\left(\sum_{j=1}^{c} \omega_{j} q_{j}\right)+\mathbf{z}^{\prime} \boldsymbol{\varphi}
$$

where $\omega_{j}$ is the weight parameter $\left(\omega_{j} \in[0,1], \sum_{j=1}^{c} \omega_{j}=1\right)$ for the $j^{\text {th }}$ component with quantile score $q_{j}$, adjusted for covariates in $\mathbf{Z}$ with corresponding parameters in $\boldsymbol{\varphi}$. The generalized link function $g($.$) relates the mean, \mu$, to the predictor variables in the right hand side of the equation, and enables the accomodation of a variety of response variables. The unknown parameters are estimated for $b=1, \ldots, B$ bootstrap samples, and the component weights are used to estimate the weighted quantile sum index, $W Q S=\sum_{j=1}^{c} \bar{\omega}_{j} q_{j}$, where $\bar{\omega}_{j}=\frac{1}{B} \sum_{b=1}^{B} \omega_{j(b)} f\left(\hat{\beta}_{1(b)}\right)$, and $f\left(\hat{\beta}_{1(b)}\right)$ is a prespecified signal function defined to place higher relative weight on samples with stronger signal. The significance of $W Q S$ is then determined using the model $g(\mu)=\beta_{0}+\beta_{1} W Q S+\mathbf{z}^{\prime} \boldsymbol{\varphi}$.

LWQS extends the traditional WQS model (5.1) to a model in which the unknown parameters vary over observations and estimates an intercept, mixture effect, vector of exposure weights, 
and vector of covariate parameters for each location. Spatial references (e.g., coordinate locations) are required for each observation in order to enable the specification of spatial structure within the model. In this work we consider data in which each observation corresponds to a unique location (i.e., one-to-one correspondence).

The LWQS method includes a variable subspace approach as a potential strategy for variable selection and as an alternative to the bootstrap sampling used to estimate the exposure weights in traditional WQS regression. The use of variable subsets (per the variable subspace approach) enables the estimation of effects for several different combinations of exposures; each of which may reflect a unique degree of systematic spatial variation. In this body of work, we present two approaches for the estimation of the final LWQS parameters. The first is a best subset estimate that minimizes model AIC over the variable subspace. The second is an ensemble estimate calculated as weighted average of estimates from subsets of a fixed size.

\subsection{The local WQS model}

Suppose that for each observation $i=1, \ldots, \mathrm{N}$, we have $c$ environmental exposures scored into quartiles denoted by $q_{j i}$, where $q_{j i}=0,1,2$, or 3 , for the $j=1, \ldots, c$ exposures.

The LWQS model for location $i$ is given by

$$
y_{i}=\beta_{0 i}+\beta_{1 i} \sum_{j} \omega_{j i} q_{j i}+\mathbf{z}_{i}^{\prime} \boldsymbol{\varphi}_{i}+\varepsilon_{i},
$$

where $y_{i}$ is the continuous response variable at location $i ; \beta_{0 i}$ and $\beta_{1 i}$ denote the intercept and mixture effect, respectively, at location $i ; \omega_{j i}$ is the weight parameter $\left(0 \leq \omega_{j i} \leq 1, \sum_{j} \omega_{j i}=1\right)$ for the $j^{\text {th }}$ component with quantile score $q_{j i}$ at location $i ; \mathbf{z}_{i}$ is the vector of covariates with 
corresponding regression coefficients $\boldsymbol{\varphi}_{i}$ at location $i$; and $\varepsilon_{i}$ is the random error term for the $i^{\text {th }}$ location. For each location $i=1, \ldots, \mathrm{N}$, we estimate the unknown parameter vector

$$
\boldsymbol{\theta}_{i}=\left[\beta_{0 i}, \beta_{1 i}, \omega_{1 i}, \ldots, \omega_{(c-1) i}, \varphi_{1 i}, \ldots, \varphi_{k i}\right]^{\prime}
$$

resulting in an $\mathrm{N} \times p$ matrix of parameter estimates $\hat{\boldsymbol{\theta}}$, with each row corresponding to the $p$ dimensional vector of estimates for a single location.

Inspired from the spatial statistical literature, weights are applied to the data in order to specify a spatial structure in the model. We define an $\mathrm{N} \times \mathrm{N}$ diagonal weight matrix $\mathbf{W}_{i}$ for location $i$, using a kernel function that typically returns a value inversely related to the distance between observations. The kernel function is dependent upon a bandwidth parameter that controls the spatial range and decay of the kernel. Examples of commonly used kernel functions include the Gaussian and the exponential, both of which decrease monotonically as a function of distance. The form of the kernel function must be chosen prior to model estimation, and the bandwidth parameter is estimated from the data using a corrected version of the Akaike Information Criterion (AIC), a measure of model goodness-of-fit. For the remainder of this document, we limit our discussion to the exponential kernel function defined as

$$
w_{i j}=\exp \left(-\frac{d_{i j}}{\gamma}\right)
$$

where $w_{i j}$ is the weight for data at location $j$ in the model estimated for location $i, d_{i j}$ is the distance between observations $i$ and $j$, and $\gamma$ is the bandwidth parameter to be estimated from the data. 


\subsubsection{Model estimation}

The estimated parameter vector $\hat{\boldsymbol{\theta}}_{i}$ for a single location $i$ is calculated as the solution that minimizes the weighted sum of square errors

$$
\mathbf{S}\left(\boldsymbol{\theta}_{i}\right)=(\mathbf{y}-\boldsymbol{\mu})^{\prime} \mathbf{W}_{i}(\mathbf{y}-\boldsymbol{\mu})
$$

where $\mu_{i}=\beta_{0 i}+\beta_{1 i} \mathbf{q}_{i}^{\prime} \boldsymbol{\omega}_{i}+\mathbf{z}_{i}^{\prime} \boldsymbol{\varphi}_{i}$, and $\mathbf{W}_{i}=\operatorname{diag}\left(w_{i 1}, \ldots, w_{i N}\right)$ is the diagonal matrix of spatial weights calculated for each location $i$ prior to the estimation. Simultaneous estimation of $\beta_{0 i}$, $\beta_{1 i}$, unknown weights $\boldsymbol{\omega}_{i}^{\prime}$, and the covariate parameters in $\boldsymbol{\varphi}_{i}^{\prime}$ is achieved through the use of an optimization algorithm that minimizes the sum of square errors defined in equation (5.5), subject to the linear constraint $\sum_{j=1}^{c} \omega_{j i}=1$ and the bounds $\omega_{j i} \in[0,1]$. We currently perform the nonlinear optimization in R using the function solnp found in the package Rsolnp. The algorithm employed belongs to the class of indirect solvers, and implements the augmented Lagrange multiplier method with a sequential quadratic programming interior algorithm.

\subsubsection{Variable subspace approach}

Local models are estimated over several variable subsets in an effort to perform variable selection and in an attempt to decorrelate the data. Here, "variable subset" refers to subsets of the $c$ components considered in the index. Model covariates are not subject to variable subsetting, and all models are adjusted for the same pre-specified vector of covariates.

Suppose we consider $b=1, \ldots, \mathrm{B}$ variable subsets $\mathrm{S}_{\mathrm{b}}$, of a fixed size $c^{*} \leq c-1$. The model for the $b^{\text {th }}$ variable subset $\mathrm{S}_{\mathrm{b}}$ at location $i$ adjusted for covariates $\mathbf{z}_{\mathrm{i}}$ is given by

$$
y_{i}=\beta_{0 i}+\beta_{1 i} \sum_{j \in S_{b}} \omega_{j i} q_{j i}+\mathbf{z}_{i}^{\prime} \boldsymbol{\varphi}_{i}+\varepsilon_{i}
$$


where $0 \leq \omega_{j i} \leq 1$, and $\sum_{j} \omega_{j i}=1$. For notational consistency, we define the matrix of local parameters for the $b^{\text {th }}$ variable subset $\mathrm{S}_{\mathrm{b}}$ as the $\mathrm{N} \times p$ matrix

$$
\boldsymbol{\theta}^{(b)}=\left[\begin{array}{c}
\boldsymbol{\theta}_{1}^{(b)} \\
\vdots \\
\boldsymbol{\theta}_{N}^{(b)^{\prime}}
\end{array}\right]=\left[\begin{array}{cccccc}
\beta_{01} & \beta_{11} & \omega_{11} & \cdots & \omega_{c 1} & \boldsymbol{\varphi}_{1}^{\prime} \\
\vdots & \vdots & \vdots & \vdots & \vdots & \vdots \\
\beta_{0 N} & \beta_{1 N} & \omega_{1 N} & \cdots & \omega_{c N} & \boldsymbol{\varphi}_{N}^{\prime}
\end{array}\right],
$$

where $\omega_{j i}=0$ for all $j \notin \mathrm{S}_{\mathrm{b}}$.

The model in (5.6) is estimated over a range of candidate bandwidth values indexed by $\gamma$, and the final estimate for the $b^{\text {th }}$ variable subset is selected based on the corrected AIC. If we let $\hat{\boldsymbol{\theta}}^{(b, \gamma)}$ denote the matrix of parameter estimates for the $b^{\text {th }}$ variable subset with bandwidth value $\gamma$, then $\hat{\boldsymbol{\theta}}_{i}^{(b, \gamma)}$ is the solution that minimizes the sum of square errors

$$
\mathbf{S}\left(\boldsymbol{\theta}_{i}^{(b)}\right)=\left(\mathbf{y}-\boldsymbol{\mu}^{(b)}\right)^{\prime} \mathbf{W}_{i}^{\gamma}\left(\mathbf{y}-\boldsymbol{\mu}^{(b)}\right)
$$

where $\mathbf{W}_{i}^{\gamma}$ is the weight matrix for the $i^{\text {th }}$ location calculated for bandwidth $\gamma$. The use of the superscript $\gamma$ in both the weight matrix and subsequent estimate denote the model's dependency on the current bandwidth value.

Analogous to the corrected AIC proposed to evaluate geographically weighted regression (GWR) models (Fotheringham et al., 2002), we define the corrected AIC as

$$
\mathrm{AICc}=2 N \log (\hat{\sigma})+N \log (2 \pi)+N\left(\frac{N+\operatorname{trace}(\mathbf{H})}{N-2-\operatorname{trace}(\mathbf{H})}\right)
$$

where $\hat{\sigma}^{2}$ is the estimated error variance given by

$$
\hat{\sigma}^{2}=\sum_{i=1}^{N}\left(y_{i}-\hat{y}_{i}\right)^{2} /\left(N-\left(2 \operatorname{trace}(\mathbf{H})-\operatorname{trace}\left(\mathbf{H}^{T} \mathbf{H}\right)\right)\right) \text {. }
$$


The estimates of the response variable $\hat{y}_{i}$ are calculated as

$$
\hat{y}_{i}=\hat{\beta}_{0 i}+\hat{\beta}_{1 i} \sum_{j \in S_{b}} \hat{\omega}_{j i} q_{j i}+\left.\mathbf{z}_{i}^{\prime} \hat{\boldsymbol{\varphi}}_{i}\right|_{\hat{\boldsymbol{\theta}}=\hat{\boldsymbol{\theta}}^{(b, \gamma)}},
$$

and $\mathbf{H}$ denotes the hat matrix with $i^{\text {th }}$ row defined as

$$
\mathbf{H}_{i}=\mathbf{D}_{i}\left(\mathbf{D}^{T} \mathbf{W}_{i} \mathbf{D}\right)^{-1} \mathbf{D}^{T} \mathbf{W}_{i},
$$

where $\mathbf{D}$ is the $\mathrm{N} \times \mathrm{p}$ derivative matrix

$$
\mathbf{D}(\boldsymbol{\theta})=\frac{\partial \boldsymbol{\mu}}{\partial \boldsymbol{\theta}^{\prime}}=\left[\left(\frac{\partial \mu_{i}}{\partial \theta_{r}}\right)\right] ; \quad i=1, \ldots, N, \quad r=1, \ldots, \mathrm{p}
$$

evaluated using the data and the parameter estimate $\hat{\boldsymbol{\theta}}^{(b, \gamma)}$.

More explicitly,

$$
\mathbf{D}(\boldsymbol{\theta})=\frac{\partial \boldsymbol{\mu}}{\partial \boldsymbol{\theta}^{\prime}}=\left[\begin{array}{cccccccc}
\frac{\partial \mu_{1}}{\partial \beta_{01}} & \frac{\partial \mu_{1}}{\partial \beta_{11}} & \frac{\partial \mu_{1}}{\partial \omega_{11}} & \ldots & \frac{\partial \mu_{1}}{\partial \omega_{c 1}} & \frac{\partial \mu_{1}}{\partial \varphi_{11}} & \ldots & \frac{\partial \mu_{1}}{\partial \varphi_{K 1}} \\
\vdots & \vdots & \vdots & \ddots & \vdots & \vdots & \ddots & \vdots \\
\frac{\partial \mu_{N}}{\partial \beta_{0 N}} & \frac{\partial \mu_{N}}{\partial \beta_{1 N}} & \frac{\partial \mu_{N}}{\partial \omega_{1 N}} & \ldots & \frac{\partial \mu_{N}}{\partial \omega_{c N}} & \frac{\partial \mu_{N}}{\partial \varphi_{1 N}} & \ldots & \frac{\partial \mu_{N}}{\partial \varphi_{K N}}
\end{array}\right]
$$

with $i^{\text {th }}$ row

$$
\mathbf{D}_{i}(\boldsymbol{\theta})=\frac{\partial \mu_{i}}{\partial \boldsymbol{\theta}^{\prime}}=\left[\frac{\partial \mu_{i}}{\partial \beta_{0 i}}, \frac{\partial \mu_{i}}{\partial \beta_{1 i}}, \frac{\partial \mu_{i}}{\partial \omega_{1 i}}, \ldots, \frac{\partial \mu_{i}}{\partial \omega_{c i}}, \frac{\partial \mu_{i}}{\partial \varphi_{1 i}}, \ldots, \frac{\partial \mu_{i}}{\partial \varphi_{K i}}\right]
$$

where $\mu_{i}=\beta_{0 i}+\beta_{1 i} \sum_{j} \omega_{j i} q_{j i}+\sum_{k} z_{k i} \varphi_{k i}$. Solving for the $r=1, . ., \mathrm{p}$ derivatives yields

$$
\mathbf{D}_{i}(\boldsymbol{\theta})=\left[1, \quad \sum_{j=1}^{c} \omega_{j i} q_{j i}, \quad \beta_{1 i} q_{1 i}, \quad \ldots, \quad \beta_{1 i} q_{c i}, \quad z_{1 i}, \quad \ldots, \quad z_{K i}\right]
$$

We calculate the matrix of local parameter estimates $\hat{\boldsymbol{\theta}}^{(b, \gamma)}$ and approximate AICc (5.9) for all candidate bandwidth values $\gamma \in \Gamma$. We then retain the estimates from the model that minimizes 
the corrected AIC. More formally, the matrix of local parameter estimates for the $b^{\text {th }}$ variable subset is defined as $\hat{\boldsymbol{\theta}}^{(b)}=\hat{\boldsymbol{\theta}}^{\left(b, \gamma^{*}\right)}$, where $\gamma^{*}$ is the candidate bandwidth value that satisfies

$$
\operatorname{AICc}\left(\hat{\boldsymbol{\theta}}^{\left(b, \gamma^{*}\right)}\right)=\min _{\gamma \in \Gamma}\left\{\operatorname{AICc}\left(\hat{\boldsymbol{\theta}}^{(b, \gamma)}\right)\right\}
$$

The estimation process is performed for each of the $b=1, \ldots, \mathrm{B}$ variable subsets resulting in an $\mathrm{N} \times \mathrm{p}$ estimated parameter matrix, $\hat{\boldsymbol{\theta}}^{(b)}$, and corresponding kernel bandwidth, $\gamma^{*}$, for each subset. We now discuss two approaches to the estimation of the final LWQS parameters based on the variable subset estimates. The "best subset" approach minimizes model AICc over the variable subspace, while the "ensemble" approach calculates the final estimates as a weighted average of estimates from variable subsets of a fixed size.

\subsubsection{Best subset estimates}

The best variable subset is defined as the subset that minimizes AICc (5.9) across all subsets of

sizes $c^{*}=3, \ldots, c-1$, where $c$ is the total number of components considered. If we let $\hat{\boldsymbol{\theta}}^{c^{*}}$ denote the estimated parameter matrix from the model that minimizes AICc over all subsets of size $c^{*}$, then the best subset estimate, $\hat{\boldsymbol{\theta}}^{\text {best }}$, is given by

$$
\hat{\boldsymbol{\theta}}^{\text {best }}=\underset{\hat{\boldsymbol{\theta}}^{c^{*}}}{\arg \min }\left\{\operatorname{AICc}\left(\hat{\boldsymbol{\theta}}^{c^{*}}\right) \mid c^{*} \in\{3, \ldots, c-1\}\right\}
$$

Estimating the final LWQS parameters using the best subset approach performs variable selection in terms of the components included in the index. Components not belonging to the best subset are excluded from the model and are interpreted as having no meaningful relationship (under the specified directional constraint) with the outcome.

Calculation of the best subset estimate requires estimation of the local parameter matrix and bandwidth value for all unique variable subsets of sizes $3, \ldots, c-1$. The procedure is 
computationally intensive and may not be feasible in settings where a large number of exposures are of interest. To elucidate, we note that given $c$ components, the total number of subsets sized $c^{*}=3, \ldots, c-1$ is given by

$$
\sum_{c^{*}=3}^{c-1}\left(\begin{array}{c}
c \\
c^{*}
\end{array}\right)=\sum_{c^{*}=3}^{c-1} \frac{c !}{c^{*} !\left(c-c^{*}\right) !}
$$

where the expression inside the summation is the familiar binomial coefficient, "n choose k", with $\mathrm{n}=c$ and $\mathrm{k}=c^{*}$. Thus, consideration of a set of 10 exposures would require that the estimation process be performed for a total of 967 subsets (120 of size 3, 210 of size 4, 252 of

size 5, 210 of size 6,120 of size 7, 45 of size 8, and 10 of size 9). Likewise, a set of 15 exposures requires the consideration of 32,646 subsets, a set of 20 exposures requires the consideration of 1048364 subsets, etc.

\subsubsection{Ensemble estimates}

As an alternative to the best subset method, we present an ensemble approach in which the unknown LWQS parameters are calculated as a weighted average of the estimated parameter matrices for B variable subsets of a fixed size $c^{*}$, where $3 \leq c^{*} \leq c-1$, and $c$ is the total number of components considered. The subset size $c^{*}$ is either selected by the user a priori or estimated from the data using an evaluation process analogous to those in random forest (e.g. k-fold crossvalidation (Kuhn, 2013).

For the ensemble method, the unknown LWQS parameters are estimated using ensemble techniques associated with B variable subsets of size $c^{*}$. The ensemble estimates are defined as

$$
\overline{\boldsymbol{\theta}}=\sum_{b=1}^{B} \hat{\boldsymbol{\theta}}^{(b)} f\left(\hat{\boldsymbol{\theta}}^{(b)}\right),
$$


where $f\left(\hat{\boldsymbol{\theta}}^{(b)}\right)$ is a pre-specified signal function that depends upon the estimates from the $b^{\text {th }}$ variable subset, and has the property $\sum_{b=1}^{B} f\left(\hat{\boldsymbol{\theta}}^{(b)}\right)=1$. Analogous to the signal function in traditional WQS, the signal function in LWQS is designed to assign higher relative weight to subsets with higher signal. For LWQS, we use AICc (5.9) as the measure of signal and define the monotonic signal function

$$
f\left(\hat{\boldsymbol{\theta}}_{(b)}\right)=\exp \left(-\operatorname{AICc}^{(b)}\right) / \sum_{b=1}^{B} \exp \left(-\operatorname{AICc}^{(b)}\right)
$$

where $\mathrm{AICc}^{(b)}$ is the standardized AICc value for the $b^{\text {th }}$ variable subset. The AICc is standardized across the $\mathrm{B}$ variable subsets as

$$
\operatorname{AICc}^{(b)}=\frac{\operatorname{AICc}\left(\hat{\boldsymbol{\theta}}^{(b)}\right)-\overline{\mathrm{AICc}}}{\sigma_{\mathrm{AICc}}},
$$

with mean

$$
\overline{\mathrm{AICc}}=\frac{1}{B} \sum_{b=1}^{B} \operatorname{AICc}\left(\hat{\boldsymbol{\theta}}^{(b)}\right)
$$

and variance

$$
\sigma_{\mathrm{AICc}}^{2}=\frac{1}{B} \sum_{b=1}^{B}\left(\operatorname{AICc}\left(\hat{\boldsymbol{\theta}}^{(b)}\right)-\overline{\mathrm{AICc}}\right)^{2}
$$

\subsubsection{Choice of variable subsets}

The choice of variable subsets is an open area of research, but we show in the subsequent simulation study that if computationally feasible, it may be advantageous to consider all unique subsets of size $c^{*}$ for $c^{*}=3, \ldots, c-1$. Model convergence can be difficult to achieve with subsets as small as $c^{*}=2$, and consideration of subsets this small moves away from the idea of evaluating multiple simultaneous environmental exposures. Conversely, evaluating all exposures does not allow for perturbation of the variable subspace. 
Future work is needed to evaluate the best choice of subsets/subspace. We expect that the appropriate size and number of subsets will depend on a multitude of factors including the total number of exposure variables, degree of correlation between exposures, number of exposures related to the outcome, strength of association with outcome, and the spatial pattern in exposure effects. In the simulation study that follows, we consider a set of six environmental exposures, and estimate LWQS models for all subsets of sizes 3, 4, and 5 in a preliminary attempt to evaluate the role of subset choice, and to compare the performance of the best subset and ensemble estimation methods.

\subsection{Simulation study}

We assess the performance of LWQS in a simulation study that considers six environmental exposures, $\mathrm{x} 1, \ldots, \mathrm{x} 6$, and a continuous outcome variable, y. In this study, three exposures variables, $x 1, x 3$ and $x 6$, are set to be related to an increase in the mean response. The remaining three exposures, $\mathrm{x} 2, \mathrm{x} 4$ and $\mathrm{x} 5$, are assumed to have no relationship with the outcome.

The continuous response vector, $\mathrm{y}$, is simulated through the local WQS model

$$
y_{i}=\beta_{0 i}+\beta_{1 i} \sum_{j=1}^{6} \omega_{i j} q_{i j}+\varepsilon_{i}, \quad i=1, \ldots N
$$

where $y_{i}$ is the response variable at location $i ; \beta_{0 i}$ and $\beta_{1 i}$ denote the intercept and mixture effect at location $i ; \omega_{j i}$ is the weight parameter $\left(0 \leq \omega_{j i} \leq 1, \sum_{j} \omega_{j i}=1\right)$ for the $j^{\text {th }}$ exposure variable with quartile score $q_{j i}=0,1,2$, or 3 at location $i$; and $\varepsilon_{i} \sim \mathrm{N}(0,0.1)$ is the random error term for the $i^{\text {th }}$ location.

We use a unit grid as the study area and simulate exposure and response data for a total of $\mathrm{N}=$ 500 randomly generated locations inside the study area. The study area is divided into three 
equal sized rows, with 170 locations falling in row 1 , defined as the uppermost $1 / 3$ of the study area; 169 locations falling in row 2, defined as the middle 1/3 of the study area; and 161 locations falling in row 3 , defined as the lower $1 / 3$ of the study area.

In total there are five simulated scenarios discussed in this document. Scenarios 1 through 3 assume "flat" exposures with no systematic spatial variation in concentrations, while scenario 4 and 5 assume a smooth spatial pattern in exposures $\mathrm{x} 2$, $\mathrm{x} 4$, and $\mathrm{x} 6$. In scenario 1 , the simulated exposure weights and mixture effect are constant over the study region. Scenario 2 introduces spatial variation in the exposure weights, and scenario 3 considers spatial variation in both the exposure weights and the mixture effect. For scenario 4 and 5, the mixture effect varies systematically over the study region in a pattern similar to that of the spatially varying exposures. In scenario 4 , the exposure weights are constant over space, while in scenario 5 , the weights vary systematically over the study region in conjunction with the mixture effect. A summary of the simulation settings for each scenario is given in Appendix B (Table B1).

\subsubsection{Scenario $1,2, \& 3$}

\section{Simulating environmental exposures}

In the first three scenarios, the mean exposures are constant over the study area (i.e., no systematic spatial variation in concentrations). Exposure variables $\mathrm{x} 1, \ldots, \mathrm{x} 6$ are simulated as multivariate normal with means given in Table 5.1 and standard deviations of 0.5. The simulated exposure means for scenarios 1 through 3 are mapped over the study area in Figure 5.1.

Table 5.1: Simulated mean exposure concentrations $(\mathrm{SD}=0.5)$ for the six exposure variables in scenario 1,2 , and 3 .

\begin{tabular}{lrrrrrr} 
Exposure: & $\mathrm{x} 1$ & $\mathrm{x} 2$ & $\mathrm{x} 3$ & $\mathrm{x} 4$ & $\mathrm{x} 5$ & $\mathrm{x} 6$ \\
\hline Mean & 5.0 & 1.5 & 2.5 & 4.0 & 3.0 & 6.0 \\
\hline
\end{tabular}




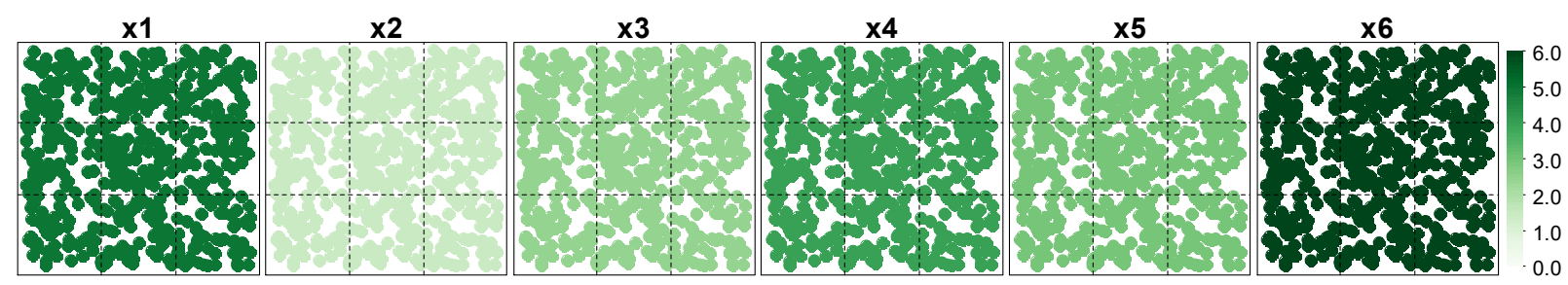

Figure 5.1: Simulated mean exposure concentrations for scenario 1, 2, and 3. Exposures are simulated as multivariate normal with standard deviations of 0.5 , and means of $5.0,1.5,2.5,4.0,3.0$, and 6.0 for $\mathrm{x} 1, \mathrm{x} 2, \mathrm{x} 3, \mathrm{x} 4, \mathrm{x} 5$, and $\mathrm{x} 6$, respectively.

Within each of the first three scenarios, we consider multiple correlation structures (of increasing complexity) for the relationships among the exposure variables. The correlation structures considered within each scenario are as follows:

Scenario 1:
a) Independent exposures
b) $r(x 1, x 3)=0.6$
c) Environmental correlation structure 1 (Table 5.2), based on the observed exposure patterns of three PCBs, two PAHs, and one pesticide in the NCI-SEER NHL study.

Scenario 2:

a) Independent exposures

b) $\mathrm{r}(\mathrm{x} 1, \mathrm{x} 3)=0.6$

c) Environmental correlation structure 2 (Table 5.3), based on the observed environmental exposures of three PCBs, one pesticide, and two PAHs in the NCI-SEER NHL study.

Scenario 3:

a) Environmental correlation structure 2 (Table 5.3), based on the observed environmental exposures of three PCBs, one pesticide, and two PAHs in the NCI-SEER NHL study.

b) Environmental correlation structure 3 (Table 5.4), based on the observed environmental exposure patterns of six PAHs from the NCI-SEER NHL study. 
Table 5.2: Environmental correlation structure 1 - Correlation among the observed exposures of three PCBs (PCB 153, PCB 170, PCB 180), two PAHs (Benzo(a)pyrene, Chrysene), and one pesticide (DDT) in the NCI-SEER NHL study.

\begin{tabular}{rrrrrrr} 
& $\mathrm{x} 1$ & $\mathrm{x} 2$ & $\mathrm{x} 3$ & $\mathrm{x} 4$ & $\mathrm{x} 5$ & $\mathrm{x} 6$ \\
\hline x1 & 1.00 & 0.71 & 0.77 & 0.24 & 0.29 & 0.37 \\
x2 & & 1.00 & 0.84 & 0.11 & 0.14 & 0.26 \\
x3 & & & 1.00 & 0.15 & 0.20 & 0.31 \\
x4 & & & & 1.00 & 0.91 & 0.08 \\
x5 & & & & & 1.00 & 0.16 \\
x6 & & & & & & 1.00 \\
\hline
\end{tabular}

Table 5.3: Environmental correlation structure 2 - Correlation among the observed exposures of three PCBs (PCB 153, PCB 170, PCB 180), one pesticide (DDT), and two PAHs (Benzo(a)pyrene, Chrysene) in the NCI-SEER NHL study.

\begin{tabular}{rrrrrrr} 
& $\mathrm{x} 1$ & $\mathrm{x} 2$ & $\mathrm{x} 3$ & $\mathrm{x} 4$ & $\mathrm{x} 5$ & $\mathrm{x} 6$ \\
\hline x1 & 1.00 & 0.71 & 0.77 & 0.37 & 0.24 & 0.29 \\
x2 & & 1.00 & 0.84 & 0.26 & 0.11 & 0.14 \\
$\mathrm{x} 3$ & & & 1.00 & 0.31 & 0.15 & 0.20 \\
x4 & & & & 1.00 & 0.08 & 0.16 \\
x5 & & & & & 1.00 & 0.91 \\
x6 & & & & & & 1.00 \\
\hline
\end{tabular}

Table 5.4: Environmental correlation structure 3 - Correlation among the observed exposures of six PAHs (Benzo(a)pyrene, Benzo(b)fluorene, Benzo(k)fluorene, Chrysene, dibenz(ah)anthracene, idenopyrene) in the NCI-SEER NHL study.

\begin{tabular}{rrrrrrr} 
& $\mathrm{x} 1$ & $\mathrm{x} 2$ & $\mathrm{x} 3$ & $\mathrm{x} 4$ & $\mathrm{x} 5$ & $\mathrm{x} 6$ \\
\hline $\mathrm{x} 1$ & 1.00 & 0.94 & 0.91 & 0.91 & 0.88 & 0.94 \\
x2 & & 1.00 & 0.94 & 0.95 & 0.88 & 0.94 \\
x3 & & & 1.00 & 0.91 & 0.87 & 0.92 \\
x4 & & & & 1.00 & 0.87 & 0.91 \\
x5 & & & & & 1.00 & 0.89 \\
x6 & & & & & & 1.00 \\
\hline
\end{tabular}

\section{Exposure weights and mixture effect}

We assume in the study design that exposure variables $\mathrm{x} 1, \mathrm{x} 3$, and $\mathrm{x} 6$ are positively associated with the outcome variable, while exposures $\mathrm{x} 2, \mathrm{x} 4$, and $\mathrm{x} 5$ have no relationship with the response. We impose that $x 1, x 3$, and $x 6$ are related to the response by assigning these exposures non-zero weights in the mixture index. Further, we impose that $\mathrm{x} 2, \mathrm{x} 4$, and $\mathrm{x} 5$ have no relationship with the response by setting their weights equal to zero. 
In scenario 1, the mixture effect and exposure weights are fixed over the study region with no systematic spatial variation. We assume that $\mathrm{x} 6$ is of the greatest relative importance with weight $\mathrm{w} 1=0.6$, followed by $\mathrm{x} 1$ with weight $\mathrm{w} 1=0.3$, and finally $\mathrm{x} 3$ with weight $\mathrm{w} 3=0.1$. The mixture effect b1 is set 1.0 for the entire study region. Maps of the simulated parameter values for scenario 1 are given in Figure 5.2.

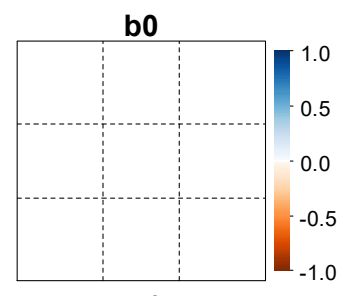

w1

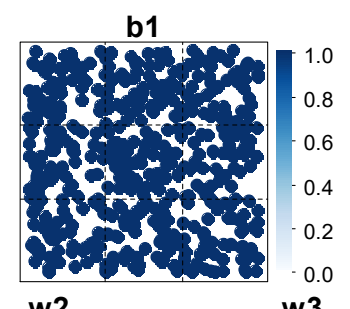

w3
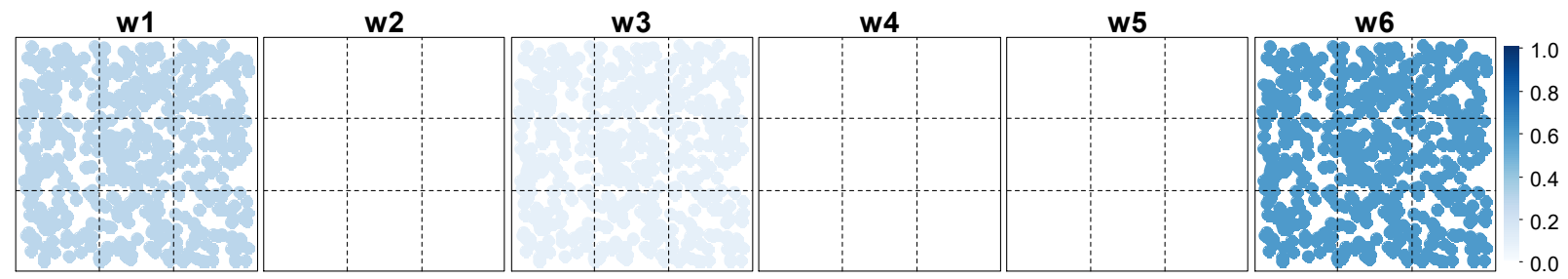

Figure 5.2: Simulated parameter values over the study region for scenario 1 .

The intercept (denoted as b0), mixture effect (denoted as b1), and exposure weights (denoted as $\mathrm{w} 1, \ldots, \mathrm{w} 6)$ are fixed over the study region with simulated values $\mathrm{b} 0=0, \mathrm{~b} 1=1, \mathrm{w} 1=0.3$, w2 $=$ $0.0, \mathrm{w} 3=0.1, \mathrm{w} 4=0.0, \mathrm{w} 5=0.0$, and $\mathrm{w} 6=0.6$.

In scenario 2, the mixture effect $\mathrm{b} 1=1$ is again fixed over the study region. However, in contrast to scenario 1 , the exposure weights for $\mathrm{x} 1$ and $\mathrm{x} 6$ now vary systematically over the study region. The weight for $\mathrm{x} 1$ is highest in the first row and lowest in the third row with values of $\omega_{1}=0.5$, 0.3 , and 0.1 in rows 1,2 , and 3, respectively. Conversely, the weight for $\mathrm{x} 6$ is lowest in the first row and highest in the third, with values of $\mathrm{w} 6=0.4,0.6$, and 0.8 in rows 1,2 , and 3 , respectively. The effect of $x 3(w 3=0.1)$ remains constant over the study region. In terms of relative importance of exposure variables, the weights impose that $\mathrm{x} 1$ is the most important exposure in the first row of the study region, while $\mathrm{x} 6$ is the most important in the latter two rows. The simulated parameter values for scenario 2 are given in Figure 5.3. 


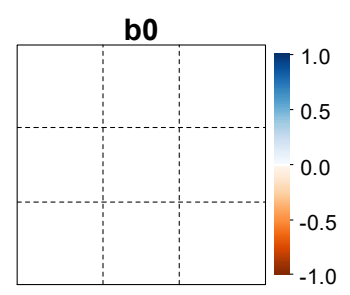

w1

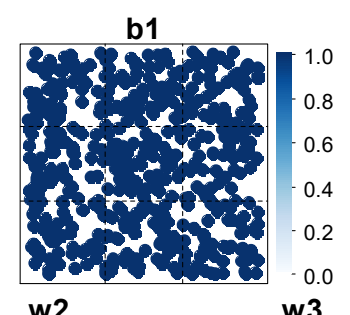

w3 w4

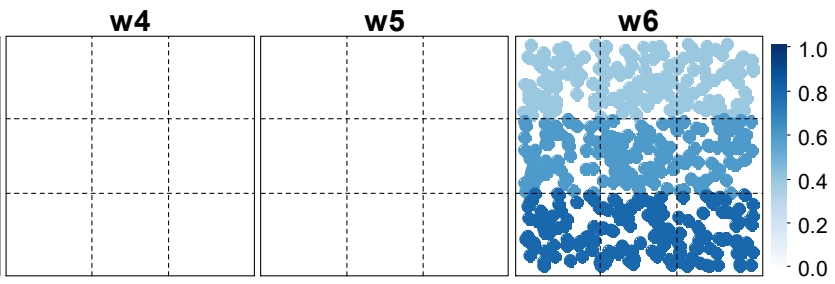

Figure 5.3: Simulated parameter values over the study region for scenario 2 .

The intercept $b 0=0$ and mixture effect $b 1=1$ are fixed over the study region. The weight for $\mathrm{x} 1$ decreases by row from the top of the study region to the bottom with values of $w 1=0.5,0.3$, and 0.1 for rows 1,2 , and 3 , respectively. The weight for $\mathrm{x} 6$ increases with descending rows with values of w6 $=0.4,0.6$, and 0.8 , in rows 1,2 , and 3 , respectively. The weight for $\mathrm{x} 3$ remains constant over the study region with $\mathrm{w} 1=0.1$.

The simulated exposure weights in scenario 3 are the same as those used in scenario 2. To recap, w1 decreases by row with values of $0.5,0.3$, and 0.1 in rows 1,2 , and 3 , respectively; w6 increases by row with values of $0.4,0.6$, and 0.8 , in rows 1,2 , and 3 , respectively; and w $1=0.1$ for the entire study region. Scenario 3 differs from the former scenario by introducing systematic spatial variation in the mixture effect. In particular, the mixture effect increases from the first to last row in the study area, with $\mathrm{b} 1=0.5,0.7$, and 1.0 in rows 1,2 , and 3 , respectively. The simulated parameter values for scenario 3 are shown in Figure 5.4. 


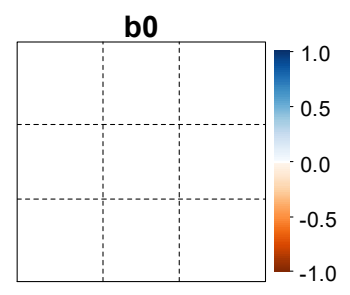

w1

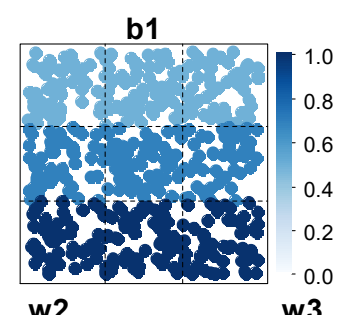

w3

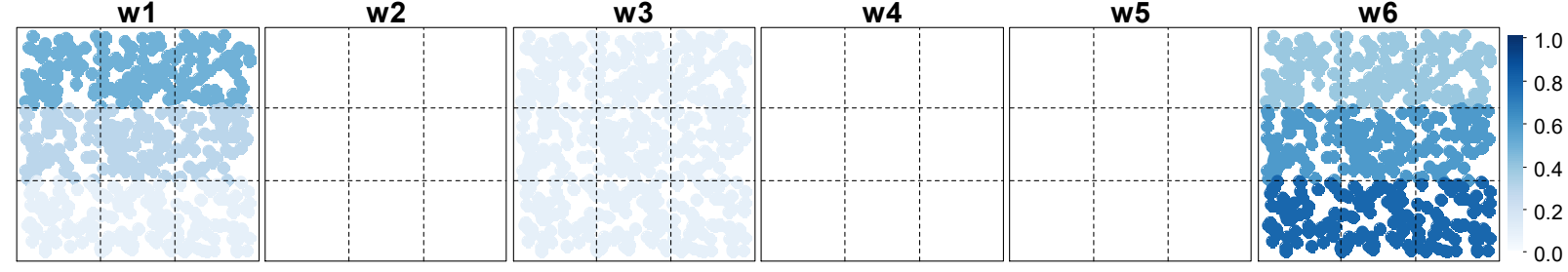

Figure 5.4: Simulated parameter values over the study region for scenario 3 .

The intercept, $\mathrm{b} 0=0$, is constant over study region. The weight for $\mathrm{x} 1$ decreases by row from the top of the study region to the bottom with values of $\mathrm{w} 1=0.5,0.3$, and 0.1 for rows 1,2 , and 3 , respectively. Conversely, the weight for $\mathrm{x} 6$ increases with descending rows with values of $\mathrm{w} 6=$ $0.4,0.6$, and 0.8 , in rows 1,2 , and 3 , respectively. The weight for $\times 3$ remains constant over the study region with $\mathrm{w} 1=0.1$. The mixture effect (b1) increases as the weight for $\mathrm{x} 6$ increases with values of $\mathrm{b} 1=0.5,0.7$, and 1.0 in rows 1,2 , and 3 , respectively.

\subsubsection{Scenario 4 \& 5}

Scenario 4 and 5 consider exposures variables with concentrations that vary smoothly over space. We measure the proximity of each location to the center of the study area, and use this distance to impose a central region of high exposures and/or high activity within the study area.

\section{Simulating environmental exposures}

For scenario 4 and 5, we assume no systematic spatial variation in exposure concentrations for variables $\mathrm{x} 1, \mathrm{x} 3$ and $\mathrm{x} 5$, while concentrations for $\mathrm{x} 2, \mathrm{x} 4$, and $\mathrm{x} 6$ decrease radially from the center of the study region (see Figure 5.5). Exposure data for $\mathrm{x} 1, \mathrm{x} 3$, and $\mathrm{x} 5$ were simulated as multivariate normal with standard deviations of 0.5 ; means of 5.0, 2.5, and 3.0, respectively; and a correlation structure of $r(x 1, x 3)=0.91, r(x 1, x 5)=0.88$, and $r(x 3, x 5)=0.87$, based on the observed correlation among three PAHs in the NCI-SEER NHL study (see Table 5.4). 
Concentrations for $\mathrm{x} 2, \mathrm{x} 4$, and $\mathrm{x} 6$ were set to vary systematically as a function of distance from the center of the study region. More specifically, for location $i$, we calculate distance from the center of the study region as

$$
d_{i}=\sqrt{\left(u_{i}-0.5\right)^{2}+\left(v_{i}-0.5\right)^{2}},
$$

where $\left(u_{i}, v_{i}\right)$ are the spatial coordinates for the $i^{\text {th }}$ location. We then simulate exposure data for $\mathrm{x} 6$ at each of the $i=1, \ldots, \mathrm{N}$ locations as $x_{6 i}=3+5 \exp \left(-2 d_{i}\right)+\varepsilon_{N(0,0.5)}$, where $\varepsilon_{N(0,0.5)}$ is a random error term with mean 0 and standard deviation of 0.5 . The remaining exposures were simulated as functions of $\mathrm{x} 6$, with concentrations given by $x_{2}=x_{6}-\varepsilon_{N(1,1)}$ and $x_{4}=\sqrt{x_{6}}+\varepsilon_{N(0,0.5)}$.

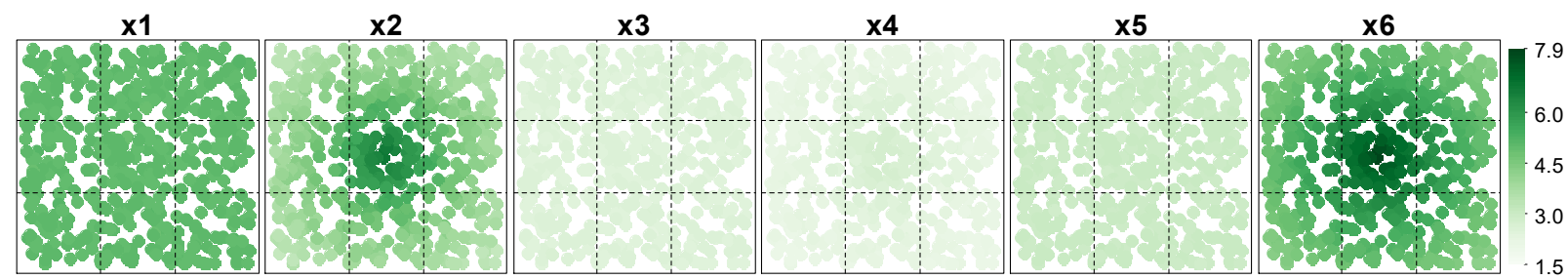

Figure 5.5: Simulated exposure concentrations for scenario 4 and 5 .

Exposure variables $\mathrm{x} 1, \mathrm{x} 3$, and $\mathrm{x} 5$ were simulated as multivariate normal with standard deviations of 0.5 ; means of 5.0, 2.5, and 3.0 respectively; and correlations of ranging from 0.87 to 0.91 . Exposure variables $\mathrm{x} 2, \mathrm{x} 4$, and $\mathrm{x} 6$ vary systematically as a function of distance from the center of the study region. Concentrations for these variables are highest in the center of the study area and decrease outwardly in a radial pattern.

\section{Mixture effect and exposure weights}

In both scenario 4 and 5, we consider a mixture effect that varies systematically over the study region in a pattern similar to that of the exposure concentrations for $\mathrm{x} 2, \mathrm{x} 4$, and $\mathrm{x} 6$. For each location, the mixture effect is simulated as

$$
\beta_{1 i}=\exp \left(-2 d_{i}\right), \quad i=1, \ldots, N,
$$


a decreasing function of the distance (5.26) from the center of the study region. As shown in Figure 5.6, b1 is strongest in the center of the study region and decreases outwardly in a radial pattern.

For scenario 4, the simulated exposure weights (see Figure 5.6) are fixed over space with values of $\omega 1=0.3, \omega 3=0.1$, and $\omega 6=0.6$. In scenario 5 , we introduce systematic spatial variation in the exposure weights by allowing $\omega 6$ to decrease in a radial pattern from the center of the study grid- reflective of the exposure pattern for $\mathrm{x} 6$. More explicitly, the weight parameter for $\mathrm{x} 6$ is simulated as $\omega 6=\exp (-d)-0.3$, where $d$ is defined as the distance from the center of the study region to each location. The remaining weight is allocated randomly between $\omega 1$ and $\omega 3$ in proportions ranging from 0.3 to 0.7 , with $\omega 1=k(1-\omega 6)$ and $\omega 3=(1-k)(1-\omega 6)$, where the coefficient $k \in[0.3,0.7]$ is a randomly chosen for each location. The simulated parameter values for scenario 5 are shown in Figure 5.7.

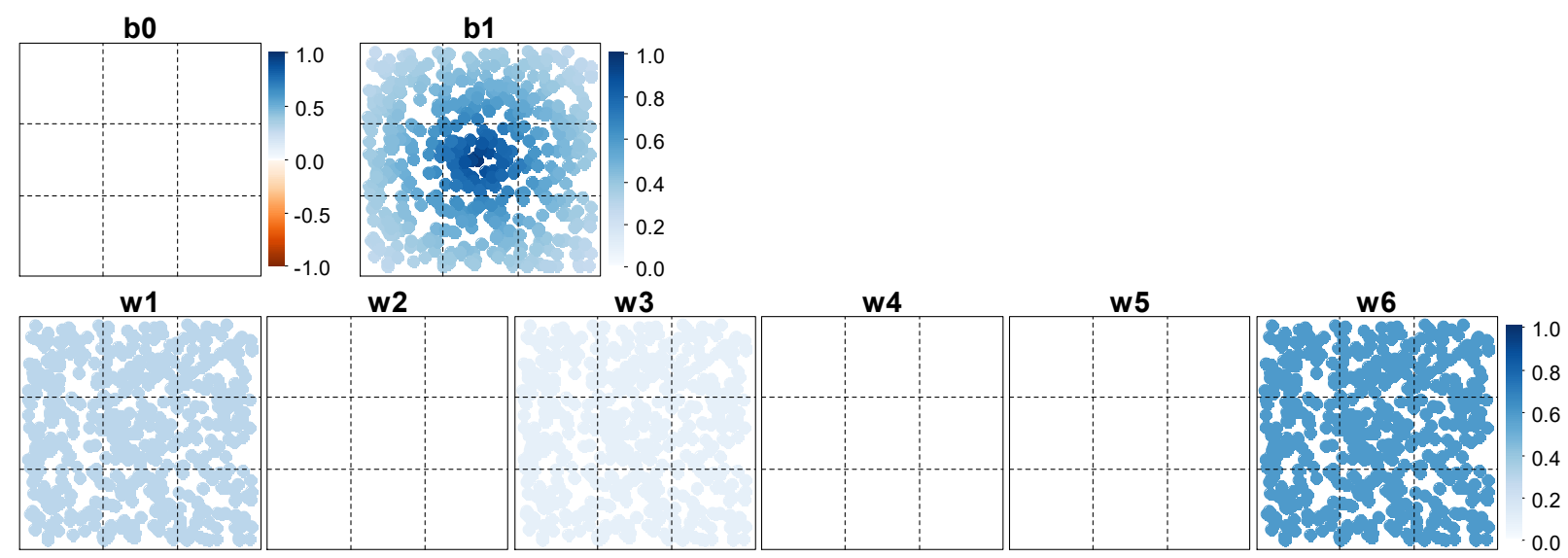

Figure 5.6: Simulated parameter values over the study region for scenario 4. The mixture effect b1 is strongest in the center of the study region and decreases outwardly in a radial pattern. The intercept, b0, and exposure weights, w1, ., w6, are fixed over the study region with simulated values $\mathrm{b} 0=0, \mathrm{w} 1=0.3, \mathrm{w} 2=0.0, \mathrm{w} 3=0.1, \mathrm{w} 4=0.0, \mathrm{w} 5=0.0$, and $\mathrm{w} 6=$ 0.6 . 

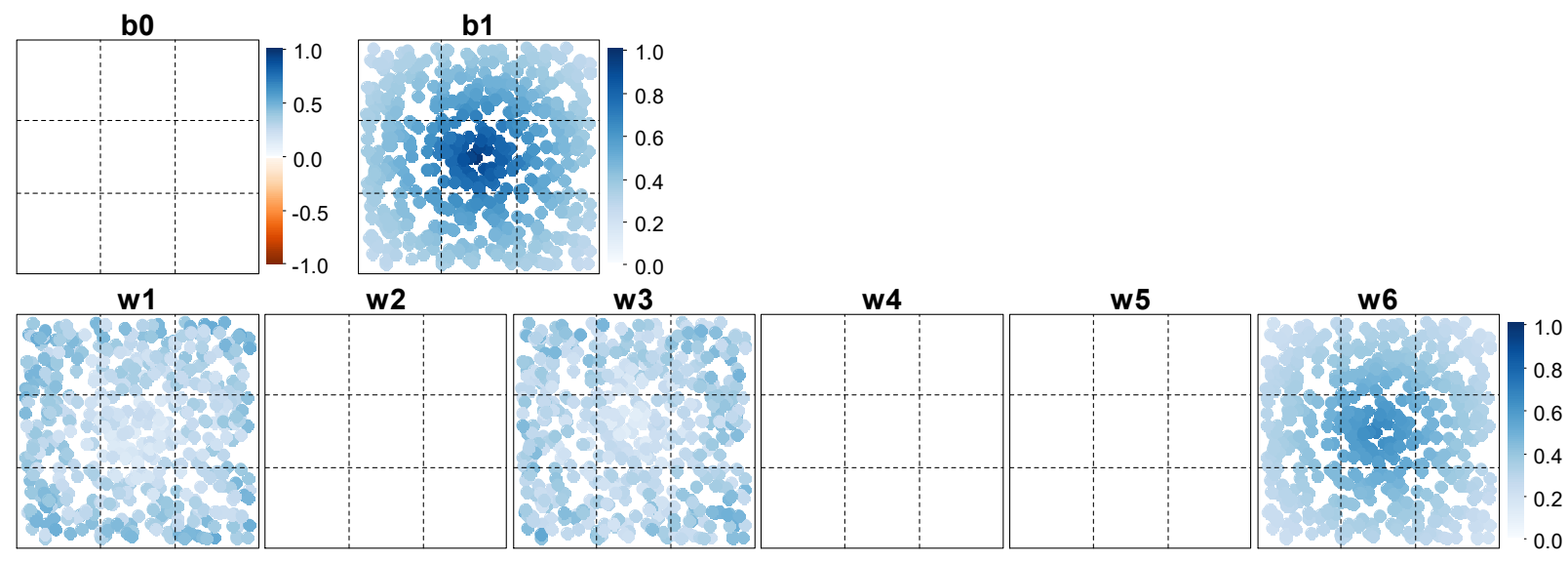

Figure 5.7: Simulated parameter values over the study region for scenario 5.

The mixture effect b1 is strongest in the center of the study region and decreases outwardly in a radial pattern. Exposure weight w6 also decreases in a radial pattern with its strongest values in the in the center of the study region. Exposure weights w1 and w3 are set to randomly share the remaining index weights, while $\mathrm{w} 2, \mathrm{w} 4$, and $\mathrm{w} 5$ are set to 0 . The intercept b0 is set to zero for the entire study region.

\subsubsection{Analysis and evaluation of LWQS}

For each simulation setting we generate a total of 100 simulated data sets and perform LWQS regression on all subsets of size 3, 4, and 5 for each data set. When estimating the LWQS models we use the exponential kernel function (5.4), and consider candidate bandwidth values of $\gamma \in\{0.1,0.2,0.4,0.5,0.6,0.8,1.0,1.4\}$. The range of candidate values for the bandwidth parameter is specified to include values small enough to accommodate strong spatial dependency and large enough to encompass the entire study region. Final LWQS parameter estimates are calculated using both the best subset and the ensemble methods. For the ensemble method, we select the final parameter estimates for the subset size that has the lowest weighted average AICc, calculated using weights determined by the signal function (5.21) described in the previous section. Results are presented in aggregate over the 100 simulated data sets for the best subset and selected ensemble estimates. 
When examining the performance of LWQS, we focus on the method's ability to correctly identify important exposures (i.e., those most strongly related to the outcome) and to detect changes in the mixture effect and weight parameters over space. The best subset method performs variable selection inherently, choosing which exposures enter the model through the selection of a best variable subset. For the ensemble estimates, a pre-specified "cut-off" value is used to select important exposures. In this work, an exposure is identified as selected if it receives an estimated ensemble weight of at least 0.05 .

The average parameter estimates are mapped over the study region and examined in comparison to maps of the true simulated parameters in an effort to visually assess the model's ability to detect spatially varying effects. We estimate bias at each location and calculate root mean square error $(\mathrm{RMSE})$ for each parameter $(r=1, \ldots, \mathrm{p})$ as

$$
\operatorname{RMSE}\left(\hat{\boldsymbol{\theta}}_{r}\right)=\sqrt{\frac{1}{N} \sum_{i=1}^{N}\left(\hat{\theta}_{r i}-\theta_{r i}\right)^{2}} .
$$

Summaries of the LWQS parameter estimates and error are presented for the study area or by row when appropriate. Finally, the bias is mapped over the study area in an effort to visualize local model performance and assess spatial patterning in the direction and magnitude of error.

\subsubsection{Results}

Results are presented separately for each simulated scenario. We briefly review the simulation settings within a scenario prior to the presentation of results. The reader is encouraged to refer to the table of summarized simulation settings (Table B1, appendix B) if further re-orientation is needed. 


\section{Scenario 1}

In scenario 1 the simulated exposure means, weight parameters $(w 1, \ldots, w 6)$, and mixture effect (b1) are constant over the study area. The simulated parameter values for scenario 1 are shown in Table 5.5. We consider three different correlation structures of increasing complexity for the relationships among exposures: a) independent exposures, b) $r(x 1, x 3)=0.6$, and c) environmental correlation pattern 1 (see Table 5.2) with correlations range from 0.08 to 0.91 .

Table 5.5: Simulated LWQS parameters for scenario 1.

\begin{tabular}{rrrrrrrr} 
b0 & b1 & w1 & w2 & w3 & w4 & w5 & w6 \\
\hline 0.0 & 1.0 & 0.3 & 0.0 & 0.1 & 0.0 & 0.0 & 0.6 \\
\hline
\end{tabular}

Local models were estimated for all subsets of size 3, 4, and 5, for each correlation case. The average AICc and corresponding estimated bandwidths for each subset are shown in Tables 5.6, 5.7, and 5.8, for correlation cases a, b, and c, respectively. We see from these tables that regardless of correlation pattern, LWQS selects the largest candidate bandwidth size $(\gamma=1.4)$ for all subset models, across all simulated data sets. The choice of the largest candidate value as the estimated bandwidth parameter is appropriate given the absence of spatial dependency in the simulated effects. Further, we see that for all correlation cases, the best subset (i.e., model with the lowest AICc) is the subset containing the three exposures (x1, x3, x6) set to be related to the response. Therefore, in terms of variable selection, the best subset method was able to correctly retain important exposures (i.e., sensitivity) and exclude unimportant exposures (i.e., specificity) for all correlation cases. 
Table 5.6: Summary of model AICc by variable subset and the corresponding number of times each candidate bandwidth value $\gamma$ was chosen over the 100 simulated data sets for scenario 1 in the case of a) independent exposures.

\# Times Selected

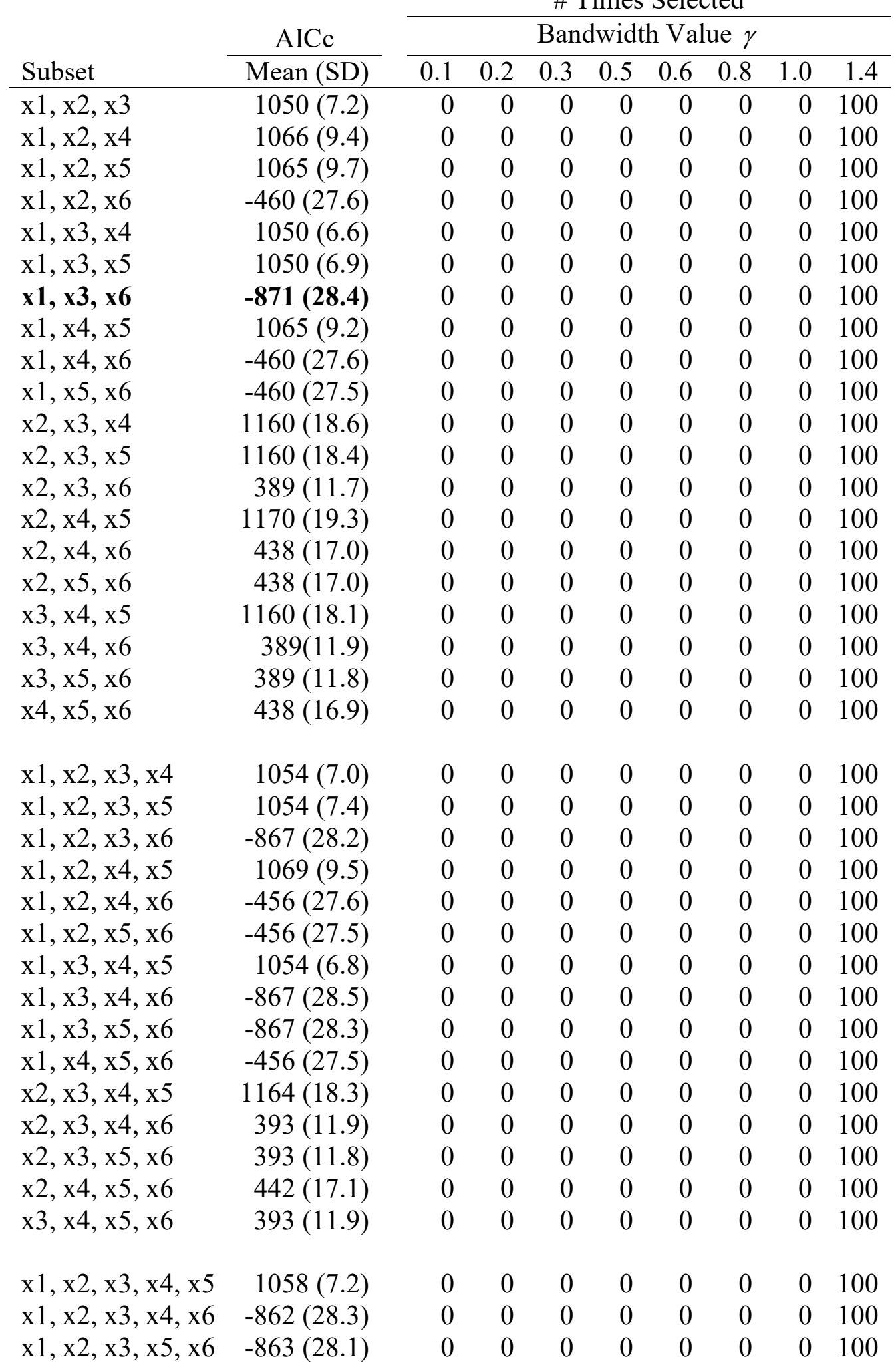




\begin{tabular}{rrrrrrrrrr}
$\mathrm{x} 1, \mathrm{x} 2, \mathrm{x} 4, \mathrm{x} 5, \mathrm{x} 6$ & $-452(27.5)$ & 0 & 0 & 0 & 0 & 0 & 0 & 0 & 100 \\
$\mathrm{x} 1, \mathrm{x} 3, \mathrm{x} 4, \mathrm{x} 5, \mathrm{x} 6$ & $-863(28.4)$ & 0 & 0 & 0 & 0 & 0 & 0 & 0 & 100 \\
$\mathrm{x} 2, \mathrm{x} 3, \mathrm{x} 4, \mathrm{x} 5, \mathrm{x} 6$ & $397(12.0)$ & 0 & 0 & 0 & 0 & 0 & 0 & 0 & 100 \\
\hline
\end{tabular}

Table 5.7: Summary of model AICc by variable subset and the corresponding number of times each candidate bandwidth value $\gamma$ was chosen over the 100 simulated data sets for scenario 1 in the case of $b) \mathrm{r}(\mathrm{x} 1, \mathrm{x} 3)=0.6$.

\begin{tabular}{|c|c|c|c|c|c|c|c|c|c|}
\hline \multirow[b]{2}{*}{ Subset } & AICc & \multicolumn{7}{|c|}{ \# Times Selected } & \\
\hline & Mean (SD) & 0.1 & 0.2 & 0.3 & 0.5 & 0.6 & 0.8 & 1.0 & 1.4 \\
\hline $\mathrm{x} 1, \mathrm{x} 2, \mathrm{x} 3$ & $1051(6.2)$ & 0 & 0 & 0 & 0 & 0 & 0 & 0 & 100 \\
\hline $\mathrm{x} 1, \mathrm{x} 2, \mathrm{x} 4$ & $1061(9.7)$ & 0 & 0 & 0 & 0 & 0 & 0 & 0 & 100 \\
\hline$x 1, x 2, x 5$ & $1062(9.1)$ & 0 & 0 & 0 & 0 & 0 & 0 & 0 & 100 \\
\hline$x 1, x 2, x 6$ & $-544(28.7)$ & 0 & 0 & 0 & 0 & 0 & 0 & 0 & 100 \\
\hline $\mathrm{x} 1, \mathrm{x} 3, \mathrm{x} 4$ & $1050(6.9)$ & 0 & 0 & 0 & 0 & 0 & 0 & 0 & 100 \\
\hline$x 1, x 3, x 5$ & $1051(6.3)$ & 0 & 0 & 0 & 0 & 0 & 0 & 0 & 100 \\
\hline$x 1, x 3, x 6$ & $-871(28.6)$ & 0 & 0 & 0 & 0 & 0 & 0 & 0 & 100 \\
\hline $\mathrm{x} 1, \mathrm{x} 4, \mathrm{x} 5$ & $1062(9.6)$ & 0 & 0 & 0 & 0 & 0 & 0 & 0 & 100 \\
\hline $\mathrm{x} 1, \mathrm{x} 4, \mathrm{x} 6$ & $-544(28.5)$ & 0 & 0 & 0 & 0 & 0 & 0 & 0 & 100 \\
\hline$x 1, x 5, x 6$ & $-544(28.7)$ & 0 & 0 & 0 & 0 & 0 & 0 & 0 & 100 \\
\hline$x 2, x 3, x 4$ & $1131(16.1)$ & 0 & 0 & 0 & 0 & 0 & 0 & 0 & 100 \\
\hline$x 2, x 3, x 5$ & $1131(15.9)$ & 0 & 0 & 0 & 0 & 0 & 0 & 0 & 100 \\
\hline$x 2, x 3, x 6$ & $240(28.0)$ & 0 & 0 & 0 & 0 & 0 & 0 & 0 & 100 \\
\hline$x 2, x 4, x 5$ & $1200(17.7)$ & 0 & 0 & 0 & 0 & 0 & 0 & 0 & 100 \\
\hline$x 2, x 4, x 6$ & $567(13.8)$ & 0 & 0 & 0 & 0 & 0 & 0 & 0 & 100 \\
\hline$x 2, x 5, x 6$ & 567 (13.9) & 0 & 0 & 0 & 0 & 0 & 0 & 0 & 100 \\
\hline$x 3, x 4, x 5$ & $1131(16.3)$ & 0 & 0 & 0 & 0 & 0 & 0 & 0 & 100 \\
\hline$x 3, x 4, x 6$ & $240(27.6)$ & 0 & 0 & 0 & 0 & 0 & 0 & 0 & 100 \\
\hline$x 3, x 5, x 6$ & $240(27.6)$ & 0 & 0 & 0 & 0 & 0 & 0 & 0 & 100 \\
\hline$x 4, x 5, x 6$ & $567(14.1)$ & 0 & 0 & 0 & 0 & 0 & 0 & 0 & 100 \\
\hline$x 1, x 2, x 3, x 4$ & $1054(6.9)$ & 0 & 0 & 0 & 0 & 0 & 0 & 0 & 100 \\
\hline$x 1, x 2, x 3, x 5$ & $1055(6.1)$ & 0 & 0 & 0 & 0 & 0 & 0 & 0 & 100 \\
\hline$x 1, x 2, x 3, x 6$ & $-867(28.8)$ & 0 & 0 & 0 & 0 & 0 & 0 & 0 & 100 \\
\hline$x 1, x 2, x 4, x 5$ & $1066(9.6)$ & 0 & 0 & 0 & 0 & 0 & 0 & 0 & 100 \\
\hline$x 1, x 2, x 4, x 6$ & $-540(28.5)$ & 0 & 0 & 0 & 0 & 0 & 0 & 0 & 100 \\
\hline$x 1, x 2, x 5, x 6$ & $-540(28.8)$ & 0 & 0 & 0 & 0 & 0 & 0 & 0 & 100 \\
\hline$x 1, x 3, x 4, x 5$ & 1055 (6.9) & 0 & 0 & 0 & 0 & 0 & 0 & 0 & 100 \\
\hline$x 1, x 3, x 4, x 6$ & $-867(28.6)$ & 0 & 0 & 0 & 0 & 0 & 0 & 0 & 100 \\
\hline$x 1, x 3, x 5, x 6$ & $-866(28.8)$ & 0 & 0 & 0 & 0 & 0 & 0 & 0 & 100 \\
\hline$x 1, x 4, x 5, x 6$ & $-540(28.6)$ & 0 & 0 & 0 & 0 & 0 & 0 & 0 & 100 \\
\hline$x 2, x 3, x 4, x 5$ & $1135(16.3)$ & 0 & 0 & 0 & 0 & 0 & 0 & 0 & 100 \\
\hline$x 2, x 3, x 4, x 6$ & 244 (27.9) & 0 & 0 & 0 & 0 & 0 & 0 & 0 & 100 \\
\hline$x 2, x 3, x 5, x 6$ & 244 (27.9) & 0 & 0 & 0 & 0 & 0 & 0 & 0 & 100 \\
\hline
\end{tabular}




\begin{tabular}{lrllllllll} 
x2, x4, x5, x6 & $571(14.0)$ & 0 & 0 & 0 & 0 & 0 & 0 & 0 & 100 \\
x3, x4, x5, x6 & $244(27.5)$ & 0 & 0 & 0 & 0 & 0 & 0 & 0 & 100 \\
& & & & & & & & & \\
x1, x2, x3, x4, x5 & $1059(6.8)$ & 0 & 0 & 0 & 0 & 0 & 0 & 0 & 100 \\
x1, x2, x3, x4, x6 & $-863(28.8)$ & 0 & 0 & 0 & 0 & 0 & 0 & 0 & 100 \\
x1, x2, x3, x5, x6 & $-862(28.9)$ & 0 & 0 & 0 & 0 & 0 & 0 & 0 & 100 \\
x1, x2, x4, x5, x6 & $-536(28.7)$ & 0 & 0 & 0 & 0 & 0 & 0 & 0 & 100 \\
x1, x3, x4, x5, x6 & $-863(28.8)$ & 0 & 0 & 0 & 0 & 0 & 0 & 0 & 100 \\
x2, x3, x4, x5, x6 & $248(27.8)$ & 0 & 0 & 0 & 0 & 0 & 0 & 0 & 100 \\
\hline
\end{tabular}

Table 5.8: Summary of model AICc by variable subset and the corresponding number of times each candidate bandwidth value $\gamma$ was chosen over the 100 simulated data sets for scenario 1 in the case of c) environmental correlation structure 1.

\# Times Selected

\begin{tabular}{|c|c|c|c|c|c|c|c|c|c|}
\hline \multirow{3}{*}{ Subset } & \multirow{3}{*}{$\frac{\text { AICc }}{\text { Average (SD) }}$} & \multirow{2}{*}{\multicolumn{8}{|c|}{ Bandwidth Value $\gamma$}} \\
\hline & & & & & & & & & \\
\hline & & 0.1 & 0.2 & 0.3 & 0.5 & 0.6 & 0.8 & 1.0 & 1.4 \\
\hline$x 1, x 2, x 3$ & $996(15.8)$ & 0 & 0 & 0 & 0 & 0 & 0 & 0 & 100 \\
\hline$x 1, x 2, x 4$ & $1008(16.6)$ & 0 & 0 & 0 & 0 & 0 & 0 & 0 & 100 \\
\hline$x 1, x 2, x 5$ & $1006(16.4)$ & 0 & 0 & 0 & 0 & 0 & 0 & 0 & 100 \\
\hline $\mathrm{x} 1, \mathrm{x} 2, \mathrm{x} 6$ & $-690(30.8)$ & 0 & 0 & 0 & 0 & 0 & 0 & 0 & 100 \\
\hline $\mathrm{x} 1, \mathrm{x} 3, \mathrm{x} 4$ & $996(15.8)$ & 0 & 0 & 0 & 0 & 0 & 0 & 0 & 100 \\
\hline $\mathrm{x} 1, \mathrm{x} 3, \mathrm{x} 5$ & $994(15.8)$ & 0 & 0 & 0 & 0 & 0 & 0 & 0 & 100 \\
\hline$\times 1, \times 3, x 6$ & $-862(30.4)$ & 0 & 0 & 0 & 0 & 0 & 0 & 0 & 100 \\
\hline $\mathrm{x} 1, \mathrm{x} 4, \mathrm{x} 5$ & $1013(17.0)$ & 0 & 0 & 0 & 0 & 0 & 0 & 0 & 100 \\
\hline $\mathrm{x} 1, \mathrm{x} 4, \mathrm{x} 6$ & $-615(31.6)$ & 0 & 0 & 0 & 0 & 0 & 0 & 0 & 100 \\
\hline$x 1, \times 5, x 6$ & $-616(31.5)$ & 0 & 0 & 0 & 0 & 0 & 0 & 0 & 100 \\
\hline$x 2, x 3, x 4$ & $1126(17.6)$ & 0 & 0 & 0 & 0 & 0 & 0 & 0 & 100 \\
\hline$x 2, x 3, x 5$ & $1120(18.0)$ & 0 & 0 & 0 & 0 & 0 & 0 & 0 & 100 \\
\hline$x 2, x 3, x 6$ & $53(35.2)$ & 0 & 0 & 0 & 0 & 0 & 0 & 0 & 100 \\
\hline$x 2, x 4, x 5$ & $1184(19.6)$ & 0 & 0 & 0 & 0 & 0 & 0 & 0 & 100 \\
\hline$x 2, x 4, x 6$ & $231(31.9)$ & 0 & 0 & 0 & 0 & 0 & 0 & 0 & 100 \\
\hline$x 2, x 5, x 6$ & $227(32.3)$ & 0 & 0 & 0 & 0 & 0 & 0 & 0 & 100 \\
\hline$x 3, x 4, x 5$ & $1127(18.1)$ & 0 & 0 & 0 & 0 & 0 & 0 & 0 & 100 \\
\hline$\times 3, x 4, x 6$ & $62(34.7)$ & 0 & 0 & 0 & 0 & 0 & 0 & 0 & 100 \\
\hline$x 3, x 5, x 6$ & $62(34.9)$ & 0 & 0 & 0 & 0 & 0 & 0 & 0 & 100 \\
\hline$x 4, x 5, x 6$ & $524(19.7)$ & 0 & 0 & 0 & 0 & 0 & 0 & 0 & 100 \\
\hline$x 1, x 2, x 3, x 4$ & $1000(15.8)$ & 0 & 0 & 0 & 0 & 0 & 0 & 0 & 100 \\
\hline$x 1, x 2, x 3, x 5$ & $998(15.7)$ & 0 & 0 & 0 & 0 & 0 & 0 & 0 & 100 \\
\hline$x 1, x 2, x 3, x 6$ & $-859(30.6)$ & 0 & 0 & 0 & 0 & 0 & 0 & 0 & 100 \\
\hline$x 1, x 2, x 4, x 5$ & $1010(16.4)$ & 0 & 0 & 0 & 0 & 0 & 0 & 0 & 100 \\
\hline$x 1, x 2, x 4, x 6$ & $-687(31.0)$ & 0 & 0 & 0 & 0 & 0 & 0 & 0 & 100 \\
\hline$x 1, x 2, x 5, x 6$ & $-687(31.0)$ & 0 & 0 & 0 & 0 & 0 & 0 & 0 & 100 \\
\hline$x 1, x 3, x 4, x 5$ & $998(15.8)$ & 0 & 0 & 0 & 0 & 0 & 0 & 0 & 100 \\
\hline
\end{tabular}




$\begin{array}{lrllllllll}\text { x1, x3, x4, x6 } & -859(30.5) & 0 & 0 & 0 & 0 & 0 & 0 & 0 & 100 \\ \text { x1, x3, x5, x6 } & -859(30.4) & 0 & 0 & 0 & 0 & 0 & 0 & 0 & 100 \\ \text { x1, x4, x5, x6 } & -611(31.6) & 0 & 0 & 0 & 0 & 0 & 0 & 0 & 100 \\ \text { x2, x3, x4, x5 } & 1124(18.0) & 0 & 0 & 0 & 0 & 0 & 0 & 0 & 100 \\ \text { x2, x3, x4, x6 } & 44(35.3) & 0 & 0 & 0 & 0 & 0 & 0 & 0 & 100 \\ \text { x2, x3, x5, x6 } & 43(35.7) & 0 & 0 & 0 & 0 & 0 & 0 & 0 & 100 \\ \text { x2, x4, x5, x6 } & 230(32.3) & 0 & 0 & 0 & 0 & 0 & 0 & 0 & 100 \\ \text { x3, x4, x5, x6 } & 64(34.8) & 0 & 0 & 0 & 0 & 0 & 0 & 0 & 100 \\ & & & & & & & & & \\ \text { x1, x2, x3, x4, x5 } & 1002(15.7) & 0 & 0 & 0 & 0 & 0 & 0 & 0 & 100 \\ \text { x1, x2, x3, x4, x6 } & -855(30.7) & 0 & 0 & 0 & 0 & 0 & 0 & 0 & 100 \\ \text { x1, x2, x3, x5, x6 } & -855(30.6) & 0 & 0 & 0 & 0 & 0 & 0 & 0 & 100 \\ \text { x1, x2, x4, x5, x6 } & -683(31.0) & 0 & 0 & 0 & 0 & 0 & 0 & 0 & 100 \\ \text { x1, x3, x4, x5, x6 } & -854(30.5) & 0 & 0 & 0 & 0 & 0 & 0 & 0 & 100 \\ \text { x2, x3, x4, x5, x6 } & 46(35.7) & 0 & 0 & 0 & 0 & 0 & 0 & 0 & 100\end{array}$

In Table 5.9, the average weighted AICc for each subset size is given along with the average AICc from the best subset, for each correlation case. For all three correlation structures, the lowest weighted AICc value corresponds to a subset of size 5. Therefore, in each case, we select as our final ensemble estimates, those calculated as the weighted average of the six subsets of size 5. We also note that for each correlation structure, the AICc from the best subset is far smaller than the weighted average AICc for each of the three subset sizes, suggesting a superior goodness-of-fit for the best subset models.

Table 5.9: Summary of model AICc in simulation scenario 1 for the best subset method and for ensemble methods using variable subset sizes of 3,4 , and 5 for the cases of a) independent exposures, b) $\mathrm{r}(\mathrm{x} 1, \mathrm{x} 3)=0.6$, and c) environmental correlation structure 1 .

\begin{tabular}{|c|c|c|c|c|}
\hline \multirow[b]{3}{*}{ Ensemble Method } & & Case a & Case b & Case c \\
\hline & & Mean (SD) & Mean (SD) & Mean (SD) \\
\hline & \# of subsets & Weighted AICc & Weighted AICc & Weighted AICc \\
\hline Size 3 & 20 & $-179(18.5)$ & $-209(19.5)$ & $-256(20.8)$ \\
\hline Size 4 & 15 & $-438(21.0)$ & $-460(22.0)$ & $-506(23.7)$ \\
\hline Size 5 & 6 & $-685(24.9)$ & $-697(25.7)$ & $-719(27.5)$ \\
\hline Best Subset & Bandwidth & AICc & $\mathrm{AICc}$ & $\mathrm{AICc}$ \\
\hline$x 1, x 3, x 6$ & 1.4 & $-871(28.4)$ & $-871(28.6)$ & $-862(30.4)$ \\
\hline
\end{tabular}


The best subset LWQS parameter estimates are mapped over the study area for the three correlation cases in Figure 5.8. The simulated parameter values are given in panel 1 as a point of reference, and as shown in panels $2-4$, the best subset estimates are indistinguishable from the simulated parameters in all correlation cases. Further, as shown in in the left hand side of Tables 5.10 and 5.11, the best subset estimates are very precise with negligible bias across all correlation cases (all bias $<=0.001$ ). Note that there is truly no bias in $w 2$, w4, and w6, as these parameters are excluded in the best subset model.

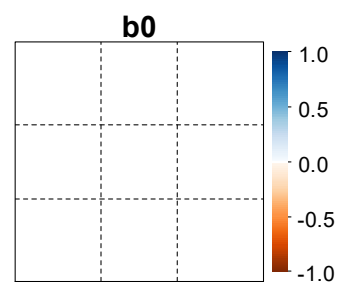

w1

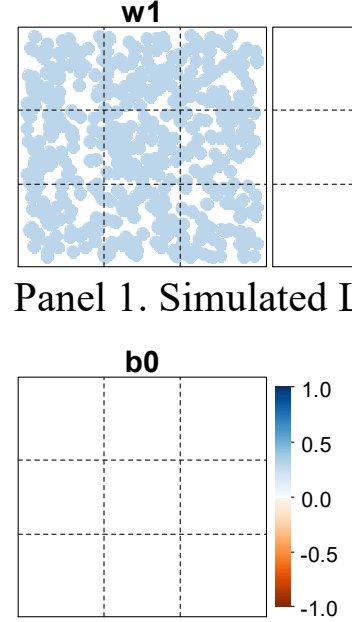

w1

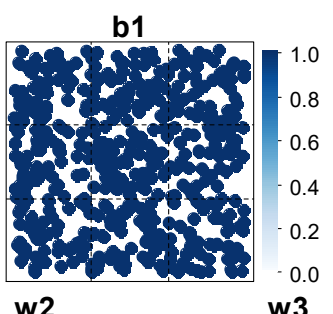

w2 w3

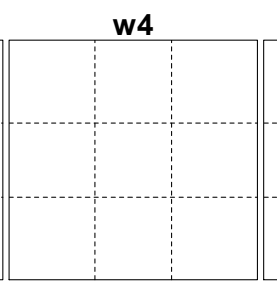

w5
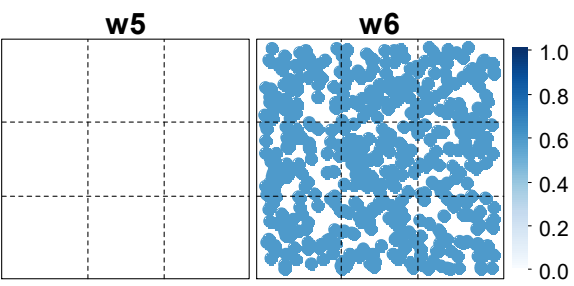

0.0

Panel 1. Simulated LWQS parameter values in scenario 1.
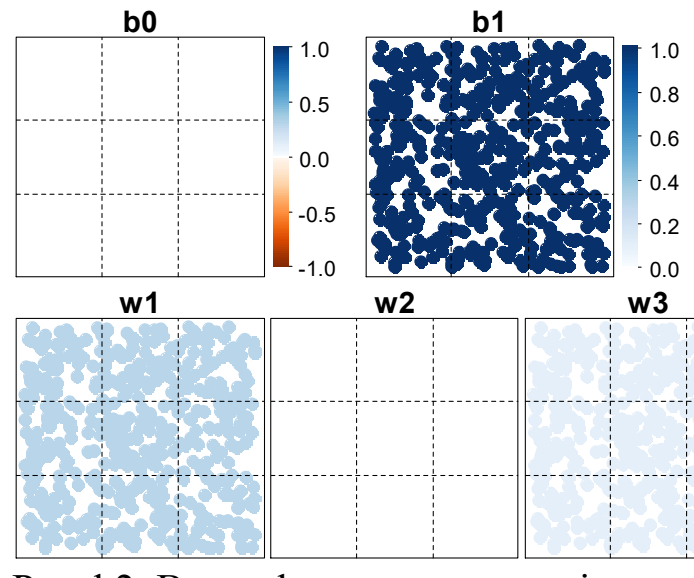

w3

w4

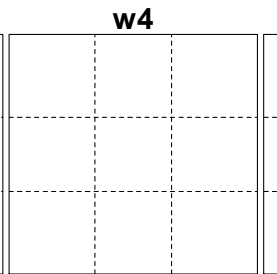

w5 w6

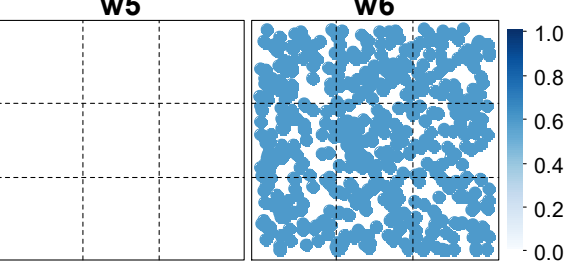

Panel 2. Best subset parameter estimates for case a) independent exposures. 

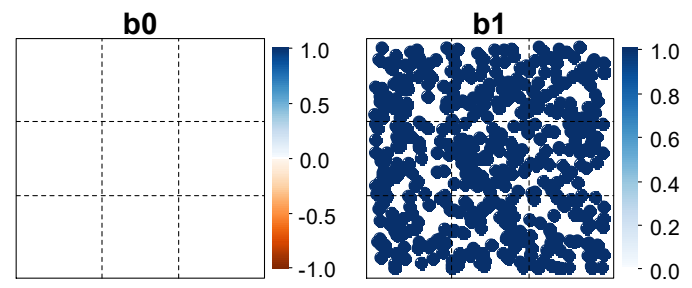

w1 w2

w3

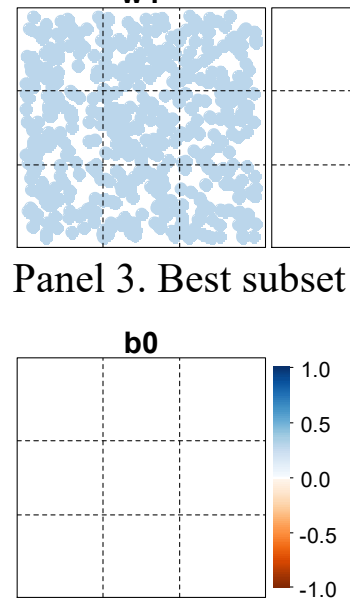

w1
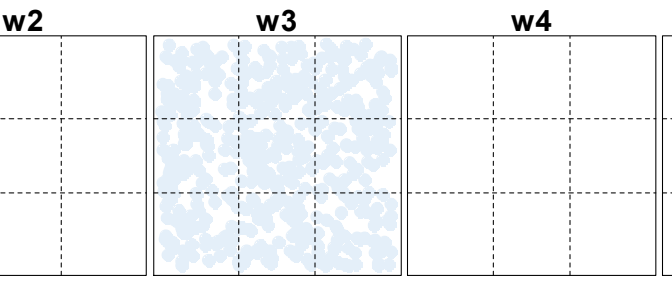

w5

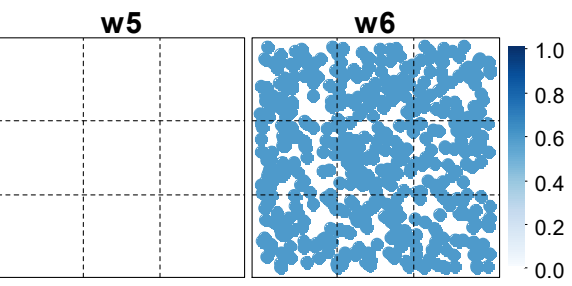

b) $r(x 1, x 3)=0.6$.

parameter estimates for case
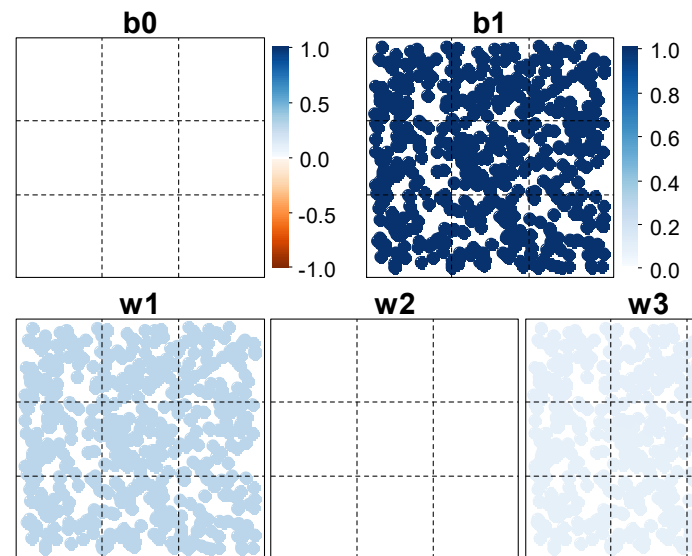

w2

w3

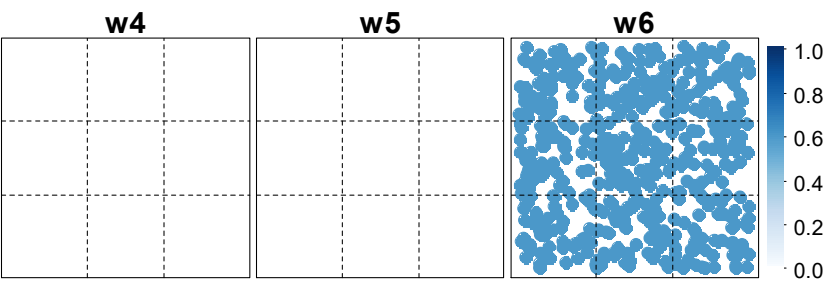

Panel 4. Best subset parameter estimates for case c) environmental correlation matrix 1.

Figure 5.8: Maps of the LWQS best subset parameter estimates in simulation scenario 1 for the cases of: a) independent exposures (panel 2); b) $r(x 1, x 3)=0.6$ (panel 3), and c) environmental correlation matrix 1 (panel 4). The simulated LWQS parameters for scenario 1 are included for the purpose of comparison (panel 1).

Table 5.10: Summary of the LWQS parameter estimates for simulation scenario 1 using the best subset ${ }^{1}$ and ensemble ${ }^{2}$ methods for the cases of a) independent exposures, b) $r(x 1, x 3)=0.6$, and c) environmental correlation structure 1 .

\begin{tabular}{lrrrrrr} 
& & \multicolumn{2}{c}{ Best Subset Estimates } & & \multicolumn{2}{c}{ Ensemble Estimates } \\
\cline { 7 - 8 } Case a & Truth & Mean & Range & & Mean & Range \\
\hline b0 & 0 & 0.00 & $(0.00,0.00)$ & & 0.06 & $(0.06,0.06)$ \\
b1 & 1 & 1.00 & $(1.00,1.00)$ & & 0.96 & $(0.96,0.96)$ \\
w1 & 0.3 & 0.30 & $(0.30,0.30)$ & & 0.30 & $(0.30,0.30)$ \\
w2 & 0 & 0 & $(0,0)$ & & 0.00 & $(0.00,0.00)$ \\
w3 & 0.1 & 0.10 & $(0.10,0.10)$ & & 0.09 & $(0.09,0.09)$ \\
w4 & 0 & 0 & $(0,0)$ & & 0.00 & $(0.00,0.00)$ \\
w5 & 0 & 0 & $(0,0)$ & & 0.00 & $(0.00,0.00)$ \\
w6 & 0.6 & 0.60 & $(0.60,0.60)$ & & 0.61 & $(0.61,0.61)$
\end{tabular}




\begin{tabular}{lrrrrr} 
Case b & & & & & \\
b0 & 0 & 0.00 & $(0.00,0.00)$ & 0.04 & $(0.04,0.04)$ \\
b1 & 1 & 1.00 & $(1.00,1.00)$ & 0.98 & $(0.97,0.98)$ \\
w1 & 0.3 & 0.30 & $(0.30,0.30)$ & 0.30 & $(0.30,0.30)$ \\
w2 & 0 & 0 & $(0,0)$ & 0.00 & $(0.00,0.00)$ \\
w3 & 0.1 & 0.10 & $(0.10,0.10)$ & 0.10 & $(0.10,0.10)$ \\
w4 & 0 & 0 & $(0,0)$ & 0.00 & $(0.00,0.00)$ \\
w5 & 0 & 0 & $(0,0)$ & 0.00 & $(0.00,0.00)$ \\
w6 & 0.6 & 0.60 & $(0.60,0.60)$ & 0.59 & $(0.59,0.59)$ \\
Case c & & & & & \\
b0 & 0 & 0.00 & $(0.00,0.00)$ & 0.01 & $(0.01,0.01)$ \\
b1 & 1 & 1.00 & $(1.00,1.00)$ & 0.99 & $(0.99,0.99)$ \\
w1 & 0.3 & 0.30 & $(0.30,0.30)$ & 0.29 & $(0.29,0.29)$ \\
w2 & 0 & 0 & $(0,0)$ & 0.02 & $(0.02,0.02)$ \\
w3 & 0.1 & 0.10 & $(0.10,0.10)$ & 0.09 & $(0.09,0.09)$ \\
w4 & 0 & 0 & $(0,0)$ & 0.00 & $(0.00,0.00)$ \\
w5 & 0 & 0 & $(0,0)$ & 0.00 & $(0.00,0.00)$ \\
w6 & 0.6 & 0.60 & $(0.60,0.60)$ & 0.59 & $(0.59,0.59)$ \\
\hline
\end{tabular}

${ }^{1}$ Best subset estimates pertain to $\mathrm{S}_{\mathrm{b}}=\{\mathrm{x} 1, \mathrm{x} 3, \mathrm{x} 6\}$ with estimated bandwidth value $\gamma=1.4$.

${ }^{2}$ Ensemble estimates are calculated using subsets of size 5.

Table 5.11: Summary of the bias and RMSE in the estimated LWQS parameters for simulation scenario 1 using the best subset ${ }^{1}$ and ensemble ${ }^{2}$ methods for the cases of a) independent exposures, b) $\mathrm{r}(\mathrm{x} 1, \mathrm{x} 3)=0.6, \mathrm{c})$ environmental correlation structure 1 .

\begin{tabular}{lrrrrrrrr} 
& \multicolumn{3}{c}{ Best Subset Estimates } & & \multicolumn{3}{c}{ Ensemble Estimates } \\
\cline { 2 - 3 } \cline { 7 - 9 } Case a & Avg. Bias & Bias Range & RMSE & & Avg. Bias & Bias Range & RMSE \\
\hline b0 & 0.00 & $(0.00,0.00)$ & 0.00 & & 0.06 & $(0.06,0.06)$ & 0.06 \\
b1 & 0.00 & $(0.00,0.00)$ & 0.00 & & -0.04 & $(-0.04,-0.04)$ & 0.04 \\
w1 & 0.00 & $(0.00,0.00)$ & 0.00 & & 0.00 & $(0.00,0.00)$ & 0.00 \\
w2 & 0 & $(0,0)$ & 0 & & 0.00 & $(0.00,0.00)$ & 0.00 \\
w3 & 0.00 & $(0.00,0.00)$ & 0.00 & & -0.01 & $(-0.01,-0.01)$ & 0.01 \\
w4 & 0 & $(0,0)$ & 0 & & 0.00 & $(0.00,0.00)$ & 0.00 \\
w5 & 0 & $(0,0)$ & 0 & & 0.00 & $(0.00,0.00)$ & 0.00 \\
w6 & 0.00 & $(0.00,0.00)$ & 0.00 & & 0.01 & $(0.01,0.01)$ & 0.01 \\
Case b & & & & & & & \\
b0 & 0.00 & $(0.00,0.00)$ & 0.00 & & 0.04 & $(0.04,0.04)$ & 0.04 \\
b1 & 0.00 & $(0.00,0.00)$ & 0.00 & & -0.02 & $(-0.03,-0.02)$ & 0.02 \\
w1 & 0.00 & $(0.00,0.00)$ & 0.00 & & 0.00 & $(0.00,0.00)$ & 0.00 \\
w2 & 0 & $(0,0)$ & 0.00 & & 0.00 & $(0.00,0.00)$ & 0.00 \\
w3 & 0.00 & $(0.00,0.00)$ & 0.00 & & 0.00 & $(0.00,0.00)$ & 0.00 \\
w4 & 0 & $(0,0)$ & 0 & & 0.00 & $(0.00,0.00)$ & 0.00 \\
w5 & 0 & $(0,0)$ & 0 & & 0.00 & $(0.00,0.00)$ & 0.00 \\
w6 & 0.00 & $(0.00,0.00)$ & 0.00 & & -0.01 & $(-0.01,-0.01)$ & 0.01
\end{tabular}




\begin{tabular}{lrrrrrr} 
Case c & & & & & & \\
b0 & 0.00 & $(0.00,0.00)$ & 0.00 & 0.01 & $(0.01,0.01)$ & 0.01 \\
b1 & 0.00 & $(0.00,0.00)$ & 0.00 & -0.01 & $(-0.01,-0.01)$ & 0.01 \\
w1 & 0.00 & $(0.00,0.00)$ & 0.00 & -0.01 & $(-0.01,-0.01)$ & 0.01 \\
w2 & 0 & $(0,0)$ & 0 & 0.02 & $(0.02,0.02)$ & 0.02 \\
w3 & 0.00 & $(0.00,0.00)$ & 0.00 & -0.01 & $(-0.01,-0.01)$ & 0.01 \\
w4 & 0 & $(0,0)$ & 0 & 0.00 & $(0.00,0.00)$ & 0.00 \\
w5 & 0 & $(0,0)$ & 0 & 0.00 & $(0.00,0.00)$ & 0.00 \\
w6 & 0.00 & $(0.00,0.00)$ & 0.00 & -0.01 & $(-0.01,-0.01)$ & 0.01 \\
\hline
\end{tabular}

${ }^{1}$ Best subset estimates pertain to $\mathrm{S}_{\mathrm{b}}=\{\mathrm{x} 1, \mathrm{x} 3, \mathrm{x} 6\}$ with estimated bandwidth value $\gamma=1.4$.

${ }^{2}$ Ensemble estimates are calculated using subsets of size 5.

Maps of the ensemble estimates from subsets of size 5 are shown for each correlation case in

Figure 5.9. As with Figure 5.8, the simulated parameter values are shown in panel 1 as a point of reference. In panels 2-4, we see evidence of positive bias in b0 across all correlation cases, and an overestimation of w2 in the case of environmental correlation matrix 1 (panel 4). From the right side of Table 5.10, it is clear that the ensemble method produces slight overestimates of the intercept, b0, and slight underestimates of the mixture effect, b1. We note however that the magnitude of the bias in $\mathrm{b} 0$ and $\mathrm{b} 1$ decreases with increasing complexity in the correlation among exposures (Table 5.11). More specifically, the magnitude of bias associated with b0 and b1 is greatest in the case of independent exposures (bias $=0.06$ and -0.04 for b0 and b1, respectively) and smallest in the most complex correlation case, i.e., correlation matrix 1 (bias $=$ 0.01 and -0.01 for $b 0$ and $b 1$, respectively). Maps of the bias in the ensemble estimates are shown in Figure 5.10. From Table 5.10 and 5.11, we see that there is slight error in the ensemble estimates of the weight parameters in each correlation case. In Figure 5.10, the bias appears most notable in w3 in the case of independent exposures (panel 1), and in w2 in the case where exposures are correlated according to environmental correlation matrix 1 (panel 3). 


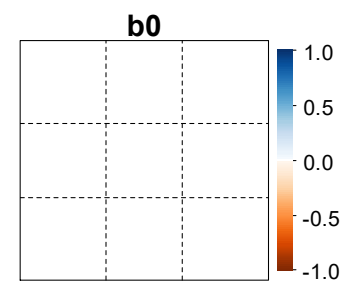

w1

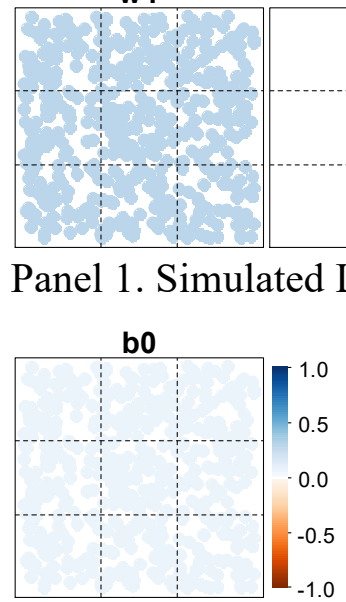

w1

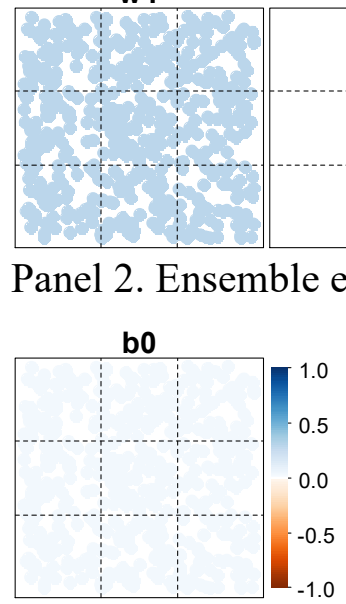

w1

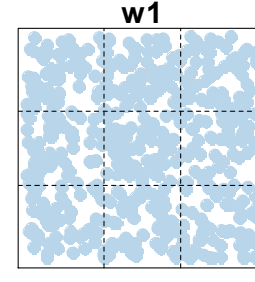

Panel 3. Ensemble estimates from subsets of size 5 for case

b1

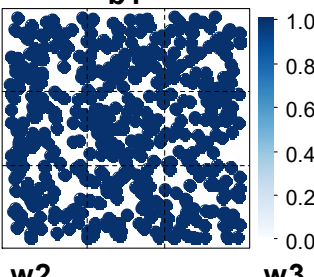

w2

w3

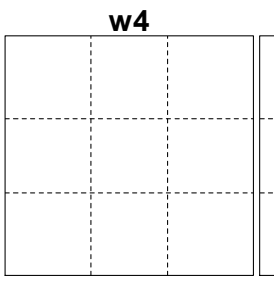

WQS parameter values in scenario 1 .

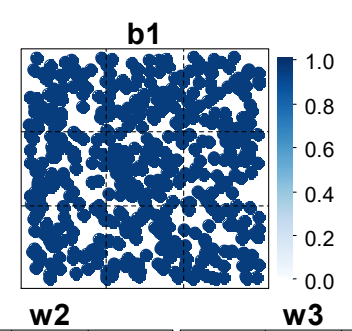

w2

w3
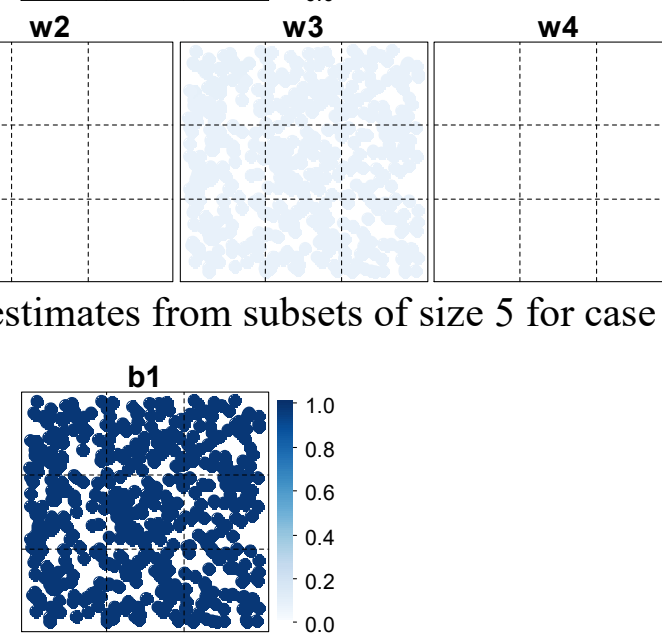

w2

w3

w5

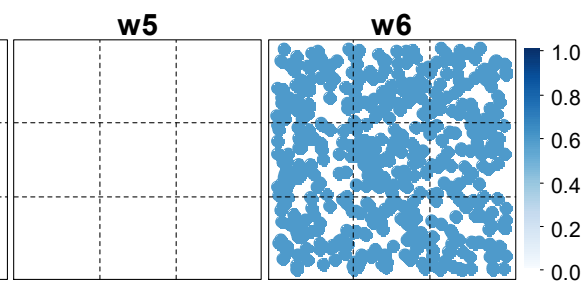
足 a

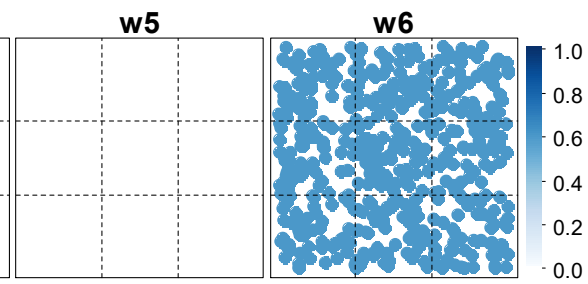

a) independent exposures.

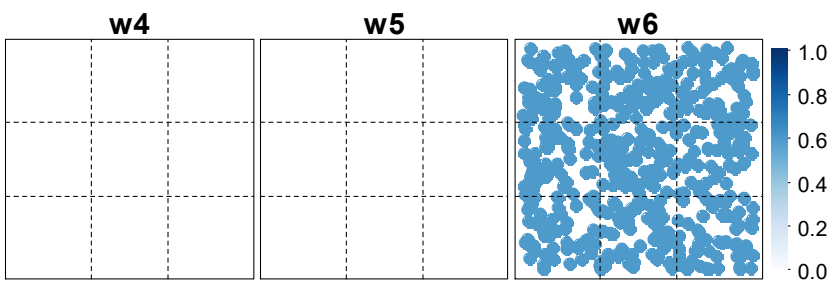

b) $r(x 1, x 3)=0.6$ 


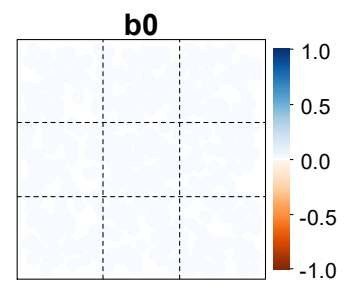

w1

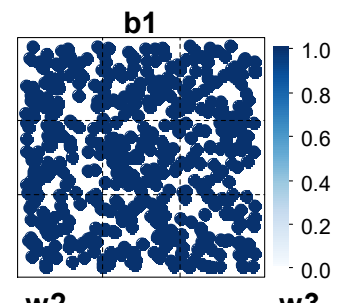

w2

w3

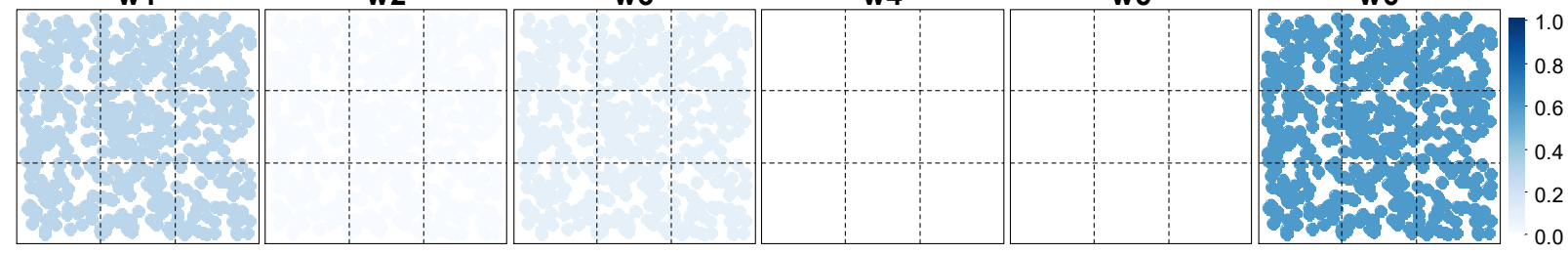

Panel 4. Ensemble estimates from subsets of size 5 for c) environmental correlation matrix 1.

Figure 5.9: Maps of the LWQS ensemble parameter estimates from subsets of size 5 in simulation scenario 1 for the correlation cases of: a) independent exposures (panel 2); $b$ ) $r(x 1, x 3$ ) $=0.6$ (panel 3), and c) environmental correlation matrix 1 (panel 4). The scenario 1 simulated LWQS parameters (panel 1) are included for the purpose of comparison.

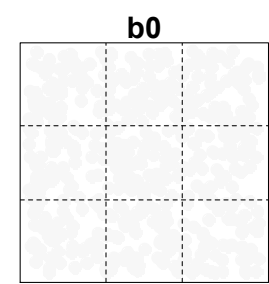

w1

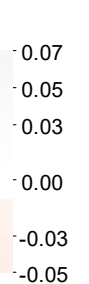

w2

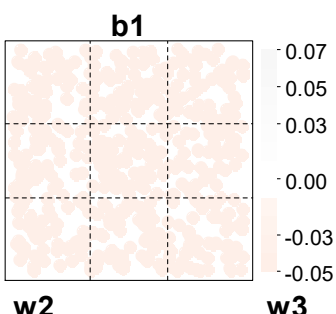

w3

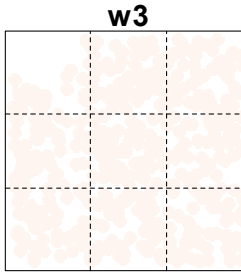

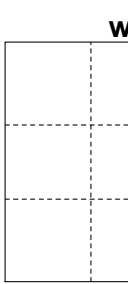

w4

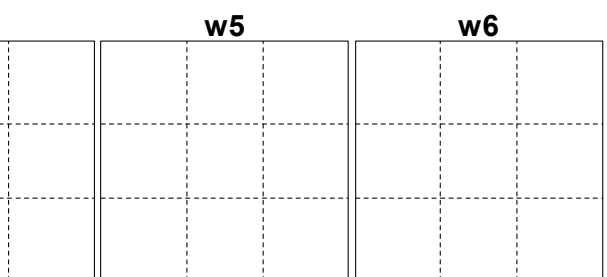

Panel 1. Bias in ensemble parameter estimates from subsets of size 5 for a) independent exposures.

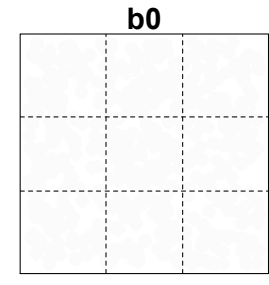

w1

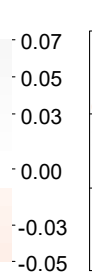

w

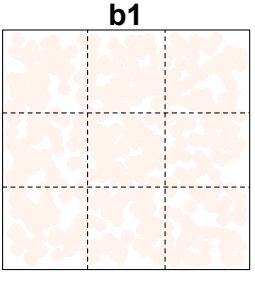

w2
-0.07
-0.05

$-0.03$

$-0.00$

$--0.03$

w3
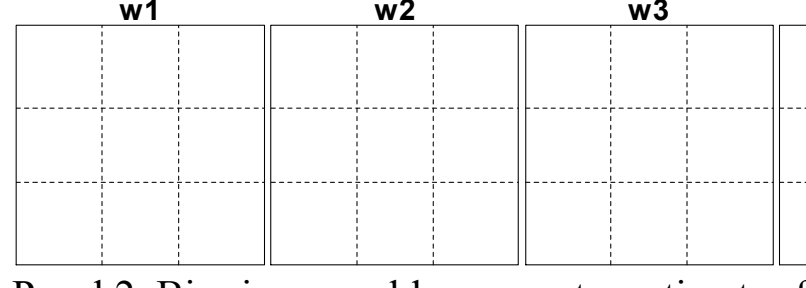

w4
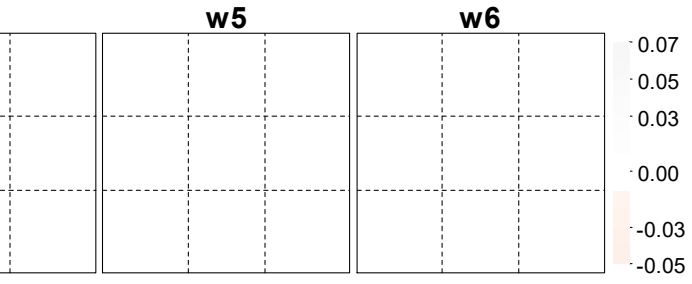

Panel 2. Bias in ensemble parameter estimates from subsets of size 5 for $b) r(x 1, x 3)=0.6$. 


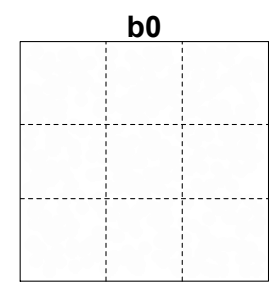

w1

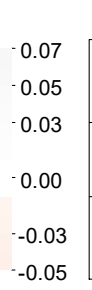

w2

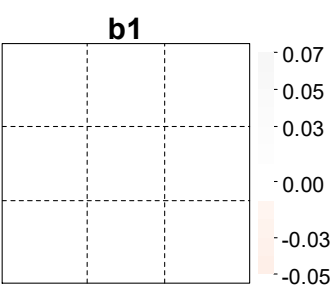

w3

w2 w3

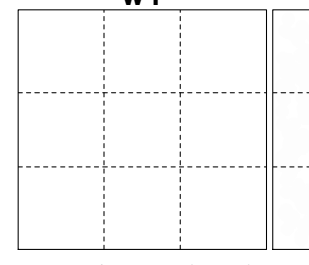

w4

w5

w6

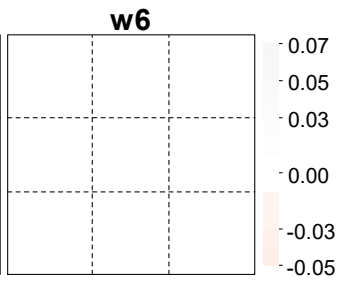

Panel 3. Bias in ensemble parameter estimates from subsets of size 5 for c) environmental correlation matrix 1.

Figure 5.10: Bias in ensemble parameter estimates from subsets of size 5 for scenario 1 in the cases of a) independent exposures (panel 1), b) $\mathrm{r}(\mathrm{x} 1, \mathrm{x} 3)=0.6$ (panel 2), and c) environmental correlation structure 1 (panel 3).

The best subset method performs decisive variable selection by choosing a best subset of exposures for inclusion in the model. In all three correlation cases, the best subset was selected to include only the important exposures. As previously mentioned, the ensemble estimates require a user defined "cut off" value to determine variable importance. When using a cut-off of 0.05 , the ensemble estimates were able to correctly classify exposures $\mathrm{x} 1, \mathrm{x} 3$, and $\mathrm{x} 6$, as important, and exposures $\mathrm{x} 2, \mathrm{x} 4$, and $\mathrm{x} 5$, as unimportant, in all correlation cases.

In scenario 1, we have shown that LWQS performs well when there is no spatial dependency in model effects or exposures, and that model performance is largely unhindered in the presence of a complex and environmentally relevant correlation pattern. Regardless of the correlation among exposures, both the best subset and ensemble methods demonstrate the ability to correctly distinguish important exposures from those unrelated to the outcome. The best subset estimates outperformed the ensemble estimates in terms of precision, producing parameter maps that were indistinguishable from maps of the simulated values in all three correlation cases. 


\section{Scenario 2}

Scenario 2 extends the simulation settings in scenario 1 to include spatially varying weight parameters and a permutation of the environmentally relevant correlation structure. More specifically, the simulated exposure means and mixture effect are constant over the study area, while the weight parameters change by row of the study region (see Table 5.12). The weight for $\mathrm{x} 1$ decreases by row from the top of the study area to the bottom. Conversely, the weight for $\mathrm{x} 6$ increases with descending rows, where it is strongest in the first and weakest in the third. The weight for $\mathrm{x} 3$ does not vary over space, with a constant value for the entirety of the study area. We again consider three correlation patterns of increasing complexity when simulating exposures: a) independent exposures, b) $r(x 1, x 3)=0.6$, and c) environmental correlation matrix 2. Environmental correlation matrix 2 (correlations ranging from 0.08 to 0.91 ) is a rearrangement of environmental correlation matrix 1, aimed at changing the intensity of correlation in the important exposures.

Table 5.12: Simulated LWQS parameters for scenario 2.

\begin{tabular}{lrrrrrrrr} 
& b0 & b1 & w1 & w2 & w3 & w4 & w5 & w6 \\
\hline Row 1 & 0.0 & 1.0 & 0.5 & 0.0 & 0.1 & 0.0 & 0.0 & 0.4 \\
Row 2 & 0.0 & 1.0 & 0.3 & 0.0 & 0.1 & 0.0 & 0.0 & 0.6 \\
Row 3 & 0.0 & 1.0 & 0.1 & 0.0 & 0.1 & 0.0 & 0.0 & 0.8 \\
\hline
\end{tabular}

Model goodness-of-fit (AICc) and choice of bandwidth parameter are summarized for all subsets of sizes 3, 4, and 5, in Tables 5.13, 5.14, and 5.15, for correlation cases a, b, and c, respectively. In all three correlation cases, the best subset (i.e., model with the lowest AICc) is correctly selected as the subset containing exposures $\mathrm{x} 1, \mathrm{x} 3$, and $\mathrm{x} 6$ (the three exposures set to be related to the response). Thus, in terms of variable selection, the best subset method was able to 
correctly retain important exposures (i.e., sensitivity) and exclude unimportant exposures (i.e., specificity) for all correlation cases.

As further shown in Tables 5.13, 5.14, and 5.15, the estimated bandwidth parameters generally reflect the degree of spatial dependency in the composition of the subset. Subset models excluding all exposures with spatially dependent parameters (e.g. subset $S_{b}=\{x 2, x 3, x 4\}$ ) select the largest candidate bandwidth value. Furthermore, subsets containing exposures $\mathrm{x} 1$ and $\mathrm{x} 6$ select small candidate bandwidth values as a consequence of the systematic spatial variation in weight parameters $\mathrm{w} 1$ and $\mathrm{w} 6$. We note that the best subset model $\left(\mathrm{S}_{\mathrm{b}}=\{\mathrm{x} 1, \mathrm{x} 3, \mathrm{x} 6\}\right)$ selects the smallest candidate bandwidth value $(\gamma=0.1)$ across all simulated data sets in all correlation cases.

Table 5.13: Summary of model AICc by variable subset and the corresponding number of times each candidate bandwidth value $\gamma$ was chosen over the 100 simulated data sets for scenario 2 in the case of a) independent exposures.

\begin{tabular}{|c|c|c|c|c|c|c|c|c|c|}
\hline \multirow[b]{3}{*}{ Subset } & \multirow{2}{*}{$\mathrm{AICc}$} & \multicolumn{8}{|c|}{ \# Times Selected } \\
\hline & & \multicolumn{8}{|c|}{ Bandwidth Value $\gamma$} \\
\hline & Mean (SD) & 0.1 & 0.2 & 0.3 & 0.5 & 0.6 & 0.8 & 1.0 & 1.4 \\
\hline $\mathrm{x} 1, \mathrm{x} 2, \mathrm{x} 3$ & $1104(13.7)$ & 0 & 0 & 0 & 6 & 6 & 12 & 8 & 68 \\
\hline $\mathrm{x} 1, \mathrm{x} 2, \mathrm{x} 4$ & $1116(16.0)$ & 0 & 0 & 0 & 5 & 1 & 9 & 8 & 77 \\
\hline$x 1, x 2, x 5$ & $1116(16.3)$ & 0 & 0 & 0 & 4 & 3 & 6 & 10 & 77 \\
\hline$x 1, x 2, x 6$ & $-158(23.7)$ & 5 & 95 & 0 & 0 & 0 & 0 & 0 & 0 \\
\hline$x 1, x 3, x 4$ & $1104(13$ & 0 & 0 & 0 & 5 & 8 & 9 & 10 & 68 \\
\hline$x 1, x 3, x 5$ & $1104(13$ & 0 & 0 & 0 & 7 & 4 & 12 & 8 & 69 \\
\hline $\mathbf{x 1 , x 3 , x 6}$ & $-452(36.8$ & 100 & 0 & 0 & 0 & 0 & 0 & 0 & 0 \\
\hline $\mathrm{x} 1, \mathrm{x} 4, \mathrm{x} 5$ & $1116(16.1)$ & 0 & 0 & 0 & 4 & 1 & 11 & 8 & 76 \\
\hline$x 1, x 4, x 6$ & $-158(24.1)$ & 5 & 95 & 0 & 0 & 0 & 0 & 0 & 0 \\
\hline$x 1, x 5, x 6$ & -158 & 8 & 92 & 0 & 0 & 0 & 0 & 0 & 0 \\
\hline $\mathrm{x} 2, \mathrm{x} 3, \mathrm{x} 4$ & $1213(18.4)$ & 0 & 0 & 0 & 0 & 0 & 0 & 0 & 100 \\
\hline $\mathrm{x} 2, \mathrm{x} 3, \mathrm{x} 5$ & $1213(18.2)$ & 0 & 0 & 0 & 0 & 0 & 0 & 0 & 100 \\
\hline$x 2, x 3, x 6$ & $582(19.6)$ & 0 & 0 & 51 & 47 & 2 & 0 & 0 & 0 \\
\hline$x 2, x 4, x 5$ & $1222(20.0)$ & 0 & 0 & 0 & 0 & 0 & 0 & o & 100 \\
\hline$x 2, x 4, x 6$ & $619(21.7)$ & 0 & 0 & 31 & 62 & 7 & 0 & 0 & 0 \\
\hline$x 2, x 5, x 6$ & $618(22.1)$ & 0 & 0 & 32 & 59 & 9 & 0 & 0 & 0 \\
\hline$\times 3, x 4, \times 5$ & $1213(18.1)$ & 0 & 0 & 0 & 0 & 0 & 0 & 0 & 100 \\
\hline$\times 3, x 4, x 6$ & $582(19.6)$ & 0 & 1 & 57 & 40 & 2 & 0 & 0 & 0 \\
\hline
\end{tabular}




\begin{tabular}{lrrrrrrrrr} 
x3, x5, x6 & $582(19.6)$ & 0 & 0 & 54 & 44 & 2 & 0 & 0 & 0 \\
x4, x5, x6 & $618(21.8)$ & 0 & 0 & 30 & 63 & 7 & 0 & 0 & 0 \\
& & & & & & & & & \\
x1, x2, x3, x4 & $1108(13.8)$ & 0 & 0 & 0 & 1 & 4 & 3 & 8 & 84 \\
x1, x2, x3, x5 & $1108(14.1)$ & 0 & 0 & 0 & 2 & 2 & 5 & 5 & 86 \\
x1, x2, x3, x6 & $-384(36.6)$ & 99 & 1 & 0 & 0 & 0 & 0 & 0 & 0 \\
x1, x2, x4, x5 & $1121(16.3)$ & 0 & 0 & 0 & 0 & 4 & 1 & 4 & 91 \\
x1, x2, x4, x6 & $-135(23.5)$ & 0 & 100 & 0 & 0 & 0 & 0 & 0 & 0 \\
x1, x2, x5, x6 & $-136(23.9)$ & 0 & 100 & 0 & 0 & 0 & 0 & 0 & 0 \\
x1, x3, x4, x5 & $1108(13.7)$ & 0 & 0 & 0 & 2 & 2 & 4 & 6 & 86 \\
x1, x3, x4, x6 & $-384(37.7)$ & 98 & 2 & 0 & 0 & 0 & 0 & 0 & 0 \\
x1, x3, x5, x6 & $-384(37.7)$ & 98 & 2 & 0 & 0 & 0 & 0 & 0 & 0 \\
x1, x4, x5, x6 & $-135(24.2)$ & 0 & 100 & 0 & 0 & 0 & 0 & 0 & 0 \\
x2, x3, x4, x5 & $1217(18.4)$ & 0 & 0 & 0 & 0 & 0 & 0 & 0 & 100 \\
x2, x3, x4, x6 & $591(20.3)$ & 0 & 0 & 15 & 72 & 9 & 3 & 1 & 0 \\
x2, x3, x5, x6 & $591(20.3)$ & 0 & 0 & 23 & 57 & 16 & 2 & 2 & 0 \\
x2, x4, x5, x6 & $626(22.1)$ & 0 & 0 & 10 & 57 & 24 & 8 & 1 & 0 \\
x3, x4, x5, x6 & $591(20.3)$ & 0 & 0 & 17 & 71 & 6 & 5 & 1 & 0 \\
& & & & & & & & & \\
x1, x2, x3, x4, x5 & $1112(14.1)$ & 0 & 0 & 0 & 0 & 2 & 2 & 1 & 95 \\
x1, x2, x3, x4, x6 & $-331(31.3)$ & 18 & 82 & 0 & 0 & 0 & 0 & 0 & 0 \\
x1, x2, x3, x5, x6 & $-331(30.8)$ & 20 & 80 & 0 & 0 & 0 & 0 & 0 & 0 \\
x1, x2, x4, x5, x6 & $-113(23.9)$ & 0 & 100 & 0 & 0 & 0 & 0 & 0 & 0 \\
x1, x3, x4, x5, x6 & $-331(31.8)$ & 22 & 78 & 0 & 0 & 0 & 0 & 0 & 0 \\
x2, x3, x4, x5, x6 & $598(20.8)$ & 0 & 0 & 3 & 51 & 31 & 9 & 4 & 2 \\
\hline
\end{tabular}

Table 5.14: Summary of model AICc by variable subset and the corresponding number of times each candidate bandwidth value $\gamma$ was chosen over the 100 simulated data sets for scenario 2 in the case of $b) r(x 1, x 3)=0.6$.

\begin{tabular}{|c|c|c|c|c|c|c|c|c|c|}
\hline \multirow[b]{3}{*}{ Subset } & \multirow[b]{2}{*}{$\mathrm{AICc}$} & \multicolumn{8}{|c|}{ \# Times Selected } \\
\hline & & \multicolumn{8}{|c|}{ Bandwidth Value $\gamma$} \\
\hline & Mean (SD) & 0.1 & 0.2 & 0.3 & 0.5 & 0.6 & 0.8 & 1.0 & 1.4 \\
\hline $\mathrm{x} 1, \mathrm{x} 2, \mathrm{x} 3$ & $1102(14.4)$ & 0 & 0 & 0 & 10 & 14 & 12 & 12 & 52 \\
\hline $\mathrm{x} 1, \mathrm{x} 2, \mathrm{x} 4$ & $1113(16.0)$ & 0 & 0 & 0 & 4 & 6 & 10 & 20 & 60 \\
\hline$x 1, x 2, x 5$ & $1113(16.1)$ & 0 & 0 & 0 & 2 & 6 & 16 & 12 & 64 \\
\hline$x 1, x 2, x 6$ & $-215(28.4)$ & 36 & 64 & 0 & 0 & 0 & 0 & 0 & 0 \\
\hline$x 1, x 3, x 4$ & $1102(14.7)$ & 0 & 0 & 0 & 8 & 12 & 14 & 10 & 56 \\
\hline$x 1, x 3, x 5$ & $1102(14.7)$ & 0 & 0 & 0 & 8 & 16 & 6 & 14 & 56 \\
\hline$x 1, \times 3, x 6$ & $-454(33.6)$ & 100 & 0 & 0 & 0 & 0 & 0 & 0 & 0 \\
\hline $\mathrm{x} 1, \mathrm{x} 4, \mathrm{x} 5$ & $1113(16.4)$ & 0 & 0 & 0 & 0 & 12 & 10 & 14 & 64 \\
\hline $\mathrm{x} 1, \mathrm{x} 4, \mathrm{x} 6$ & $-216(28.7)$ & 34 & 66 & 0 & 0 & 0 & 0 & 0 & 0 \\
\hline $\mathrm{x} 1, \mathrm{x} 5, \mathrm{x} 6$ & $-215(30.0)$ & 40 & 60 & 0 & 0 & 0 & 0 & 0 & 0 \\
\hline$x 2, x 3, x 4$ & $1182(18.7)$ & 0 & 0 & 0 & 0 & 0 & 0 & 0 & 100 \\
\hline$x 2, x 3, x 5$ & $1182(18.7)$ & 0 & 0 & 0 & 0 & 0 & 0 & 0 & 100 \\
\hline
\end{tabular}




\begin{tabular}{lrrrrrrrrr} 
x2, x3, x6 & $461(25.7)$ & 0 & 2 & 96 & 2 & 0 & 0 & 0 & 0 \\
x2, x4, x5 & $1251(21.8)$ & 0 & 0 & 0 & 0 & 0 & 0 & 0 & 100 \\
x2, x4, x6 & $713(21.1)$ & 0 & 0 & 10 & 56 & 26 & 8 & 0 & 0 \\
x2, x5, x6 & $714(21.4)$ & 0 & 0 & 8 & 58 & 18 & 16 & 0 & 0 \\
x3, x4, x5 & $1182(18.9)$ & 0 & 0 & 0 & 0 & 0 & 0 & 0 & 100 \\
x3, x4, x6 & $461(26.0)$ & 0 & 4 & 94 & 2 & 0 & 0 & 0 & 0 \\
x3, x5, x6 & $461(26.5)$ & 0 & 6 & 92 & 2 & 0 & 0 & 0 & 0 \\
x4, x5, x6 & $714(21.8)$ & 0 & 0 & 10 & 54 & 28 & 8 & 0 & 0 \\
& & & & & & & & & \\
x1, x2, x3, x4 & $1106(14.8)$ & 0 & 0 & 0 & 0 & 6 & 8 & 12 & 74 \\
x1, x2, x3, x5 & $1106(14.9)$ & 0 & 0 & 0 & 0 & 4 & 8 & 14 & 74 \\
x1, x2, x3, x6 & $-383(34.1)$ & 94 & 6 & 0 & 0 & 0 & 0 & 0 & 0 \\
x1, x2, x4, x5 & $1117(16.5)$ & 0 & 0 & 0 & 0 & 0 & 4 & 8 & 88 \\
x1, x2, x4, x6 & $-190(28.4)$ & 0 & 100 & 0 & 0 & 0 & 0 & 0 & 0 \\
x1, x2, x5, x6 & $-189(28.9)$ & 0 & 100 & 0 & 0 & 0 & 0 & 0 & 0 \\
x1, x3, x4, x5 & $1106(15.2)$ & 0 & 0 & 0 & 0 & 0 & 12 & 12 & 76 \\
x1, x3, x4, x6 & $-385(33.5)$ & 96 & 4 & 0 & 0 & 0 & 0 & 0 & 0 \\
x1, x3, x5, x6 & $-386(33.5)$ & 98 & 2 & 0 & 0 & 0 & 0 & 0 & 0 \\
x1, x4, x5, x6 & $-190(29.0)$ & 0 & 100 & 0 & 0 & 0 & 0 & 0 & 0 \\
x2, x3, x4, x5 & $1186(18.8)$ & 0 & 0 & 0 & 0 & 0 & 0 & 0 & 100 \\
x2, x3, x4, x6 & $474(25.9)$ & 0 & 0 & 74 & 26 & 0 & 0 & 0 & 0 \\
x2, x3, x5, x6 & $474(26.3)$ & 0 & 0 & 74 & 26 & 0 & 0 & 0 & 0 \\
x2, x4, x5, x6 & $720(21.8)$ & 0 & 0 & 0 & 26 & 32 & 26 & 12 & 4 \\
x3, x4, x5, x6 & $474(26.5)$ & 0 & 0 & 78 & 22 & 0 & 0 & 0 & 0 \\
x1, x2, x3, x4, x5 & $1110(15.2)$ & 0 & 0 & 0 & 0 & 0 & 2 & 4 & 94 \\
x1, x2, x3, x4, x6 & $-333(30.2)$ & 14 & 86 & 0 & 0 & 0 & 0 & 0 & 0 \\
x1, x2, x3, x5, x6 & $-332(29.7)$ & 12 & 88 & 0 & 0 & 0 & 0 & 0 & 0 \\
x1, x2, x4, x5, x6 & $-167(28.9)$ & 0 & 100 & 0 & 0 & 0 & 0 & 0 & 0 \\
x1, x3, x4, x5, x6 & $-334(29.5)$ & 12 & 88 & 0 & 0 & 0 & 0 & 0 & 0 \\
x2, x3, x4, x5, x6 & $485(26.0)$ & 0 & 0 & 44 & 54 & 2 & 0 & 0 & 0 \\
\hline
\end{tabular}

Table 5.15: Summary of model AICc by variable subset and the corresponding number of times each candidate bandwidth value $\gamma$ was chosen over the 100 simulated data sets for scenario 2 in the case of c) environmental correlation structure 2 .

\# Times Selected

\begin{tabular}{|c|c|c|c|c|c|c|c|c|c|}
\hline \multirow[b]{3}{*}{ Subset } & \multirow{3}{*}{$\frac{\mathrm{AICc}}{\text { Mean }(\mathrm{SD})}$} & \multirow{2}{*}{\multicolumn{8}{|c|}{ Bandwidth Value $\gamma$}} \\
\hline & & & & & & & & & \\
\hline & & 0.1 & 0.2 & 0.3 & 0.5 & 0.6 & 0.8 & 1.0 & 1.4 \\
\hline $\mathrm{x} 1, \mathrm{x} 2, \mathrm{x} 3$ & $1066(16.2)$ & 0 & 0 & 0 & 0 & 0 & 1 & 3 & 96 \\
\hline $\mathrm{x} 1, \mathrm{x} 2, \mathrm{x} 4$ & $1068(15.8)$ & 0 & 0 & 0 & 0 & 0 & 3 & 3 & 94 \\
\hline$x 1, x 2, x 5$ & $564(41.3)$ & 0 & 0 & 88 & 11 & 1 & 0 & 0 & 0 \\
\hline$x 1, x 2, x 6$ & $-366(34.9)$ & 74 & 26 & 0 & 0 & 0 & 0 & 0 & 0 \\
\hline$x 1, x 3, x 4$ & $1062(16.1)$ & 0 & 0 & 0 & 0 & 1 & 3 & 6 & 90 \\
\hline$x 1, x 3, x 5$ & $543(41.5)$ & 0 & 1 & 94 & 5 & 0 & 0 & 0 & 0 \\
\hline
\end{tabular}




\begin{tabular}{|c|c|c|c|c|c|c|c|c|c|}
\hline $\mathbf{x 1 , x 3 , x 6}$ & -499 (37.9) & 100 & 0 & 0 & 0 & 0 & 0 & 0 & 0 \\
\hline $\mathrm{x} 1, \mathrm{x} 4, \mathrm{x} 5$ & $562(40.9)$ & 0 & 0 & 89 & 11 & 0 & 0 & 0 & 0 \\
\hline $\mathrm{x} 1, \mathrm{x} 4, \mathrm{x} 6$ & $-309(33.1)$ & 8 & 92 & 0 & 0 & 0 & 0 & 0 & 0 \\
\hline$x 1, x 5, x 6$ & $-305(32.9)$ & 11 & 89 & 0 & 0 & 0 & 0 & 0 & 0 \\
\hline$x 2, x 3, x 4$ & $1179(18.8)$ & 0 & 0 & 0 & 0 & 0 & 0 & 1 & 99 \\
\hline$x 2, x 3, x 5$ & $740(36.1)$ & 0 & 0 & 0 & 52 & 18 & 16 & 7 & 7 \\
\hline$x 2, x 3, x 6$ & $268(37.6)$ & 0 & 38 & 62 & 0 & 0 & 0 & 0 & 0 \\
\hline$x 2, x 4, x 5$ & $801(35.3)$ & 0 & 0 & 0 & 27 & 22 & 26 & 11 & 14 \\
\hline$x 2, x 4, x 6$ & $402(32.7)$ & 0 & 3 & 93 & 4 & 0 & 0 & 0 & 0 \\
\hline$x 2, x 5, x 6$ & $424(34.4)$ & 0 & 1 & 85 & 14 & 0 & 0 & 0 & 0 \\
\hline$x 3, x 4, x 5$ & $729(34.1)$ & 0 & 0 & 1 & 52 & 19 & 12 & 9 & 7 \\
\hline$x 3, x 4, x 6$ & 278 (34.6) & 0 & 26 & 74 & 0 & 0 & 0 & 0 & 0 \\
\hline$x 3, x 5, x 6$ & $291(36.1)$ & 0 & 9 & 91 & 0 & 0 & 0 & 0 & 0 \\
\hline$x 4, x 5, x 6$ & $652(21.3)$ & 0 & 0 & 3 & 28 & 18 & 27 & 7 & 17 \\
\hline$x 1, x 2, x 3, x 4$ & $1067(16.1)$ & 0 & 0 & 0 & 0 & 0 & 0 & 0 & 100 \\
\hline$x 1, x 2, x 3, x 5$ & $556(41.0)$ & 0 & 0 & 50 & 47 & 2 & 1 & 0 & 0 \\
\hline$x 1, x 2, x 3, x 6$ & $-436(35.5)$ & 64 & 36 & 0 & 0 & 0 & 0 & 0 & 0 \\
\hline$x 1, x 2, x 4, x 5$ & 567 (40.9) & 0 & 0 & 58 & 41 & 1 & 0 & 0 & 0 \\
\hline$x 1, x 2, x 4, x 6$ & $-334(31.7)$ & 1 & 99 & 0 & 0 & 0 & 0 & 0 & 0 \\
\hline$x 1, x 2, x 5, x 6$ & $-332(31.6)$ & 0 & 100 & 0 & 0 & 0 & 0 & 0 & 0 \\
\hline$x 1, x 3, x 4, x 5$ & $547(41.0)$ & 0 & 0 & 72 & 28 & 0 & 0 & 0 & 0 \\
\hline $\mathrm{x} 1, \mathrm{x} 3, \mathrm{x} 4, \mathrm{x} 6$ & $-435(35.4)$ & 53 & 47 & 0 & 0 & 0 & 0 & 0 & 0 \\
\hline$x 1, x 3, x 5, x 6$ & $-436(35.4)$ & 54 & 46 & 0 & 0 & 0 & 0 & 0 & 0 \\
\hline$x 1, x 4, x 5, x 6$ & $-284(32.9)$ & 0 & 100 & 0 & 0 & 0 & 0 & 0 & 0 \\
\hline$x 2, x 3, x 4, x 5$ & $729(34.8)$ & 0 & 0 & 0 & 28 & 28 & 19 & 10 & 15 \\
\hline$x 2, x 3, x 4, x 6$ & $268(35.2)$ & 0 & 3 & 96 & 1 & 0 & 0 & 0 & 0 \\
\hline$x 2, x 3, x 5, x 6$ & $281(36.9)$ & 0 & 1 & 98 & 1 & 0 & 0 & 0 & 0 \\
\hline$x 2, x 4, x 5, x 6$ & $413(32.5)$ & 0 & 0 & 75 & 25 & 0 & 0 & 0 & 0 \\
\hline$\times 3, x 4, x 5, x 6$ & $289(34.1)$ & 0 & 0 & 98 & 2 & 0 & 0 & 0 & 0 \\
\hline$x 1, x 2, x 3, x 4, x 5$ & $559(40.2)$ & 0 & 0 & 25 & 65 & 9 & 1 & 0 & 0 \\
\hline$x 1, x 2, x 3, x 4, x 6$ & $-406(33.5)$ & 0 & 100 & 0 & 0 & 0 & 0 & 0 & 0 \\
\hline$x 1, x 2, x 3, x 5, x 6$ & $-406(33.4)$ & 0 & 100 & 0 & 0 & 0 & 0 & 0 & 0 \\
\hline$x 1, x 2, x 4, x 5, x 6$ & $-311(32.0)$ & 0 & 100 & 0 & 0 & 0 & 0 & 0 & 0 \\
\hline$x 1, x 3, x 4, x 5, x 6$ & $-406(33.0)$ & 0 & 100 & 0 & 0 & 0 & 0 & 0 & 0 \\
\hline$\times 2, x 3, x 4, x 5, x 6$ & $279(34.8)$ & 0 & 0 & 96 & 4 & 0 & 0 & 0 & 0 \\
\hline
\end{tabular}

In Table 5.16, the average weighted AICc for each subset size is given along with the average AICc from the best subset, for each correlation case. For all three correlation structures, the lowest weighted AICc value corresponds to a subset of size 5. Therefore, in each case, we select as our final ensemble estimates, those calculated as the weighted average of the six subsets of 
size 5. We also note that for each correlation structure, the AICc from the best subset is smaller than the weighted average AICc for each of the three subset sizes, suggesting a superior goodness-of-fit for the best subset models.

Table 5.16: Summary of model AICc in simulation scenario 2 for the best subset method and for ensemble methods using variable subset sizes of 3,4 , and 5 for the cases of a) independent exposures, $b) r(x 1, x 3)=0.6$, and c) environmental correlation structure 2 .

\begin{tabular}{lrrrrr} 
& & Case a & & Case b & Case c \\
Ensemble Method & Mean (SD) & Mean (SD) & Mean (SD) \\
\cline { 2 - 3 } & \# of subsets & Weighted AICc & & Weighted AICc & Weighted AICc \\
\hline Size 3 & 20 & $98(20.2)$ & & $73(20.5)$ & $-48(25.6)$ \\
Size 4 & 15 & $-66(25.3)$ & & $-85(24.1)$ & $-210(28.2)$ \\
Size 5 & 6 & $-208(27.2)$ & & $-220(26.8)$ & $-330(30.8)$ \\
Best Subset & & & & & \\
\hline x1, x3, x6 & Bandwidth & AICc & AICc & AICc \\
\hline
\end{tabular}

The best subset parameter estimates are mapped over the study region in Figure 5.11 for each correlation case. We see from the parameter maps that the best subset model accurately reproduces the intercept and mixture effect in all three correlation cases, with the exception of a small cluster of negatively estimated intercepts (located in the upper right corner of the study area) in the case of independent exposures. Additionally, examination of the parameter maps indicates that the best subset model is able to accurately detect the general spatial pattern in weight parameters $\mathrm{w} 1$ and $\mathrm{w} 3$ for all three correlation cases. However, in each case, the distinction between rows appears to be diminished, likely due to the use of the smooth exponential kernel function during model estimation. 


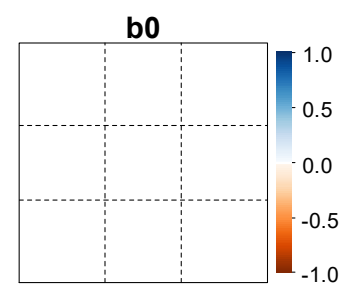

w1

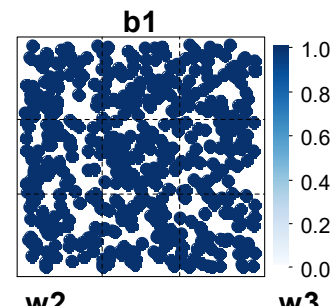

w3

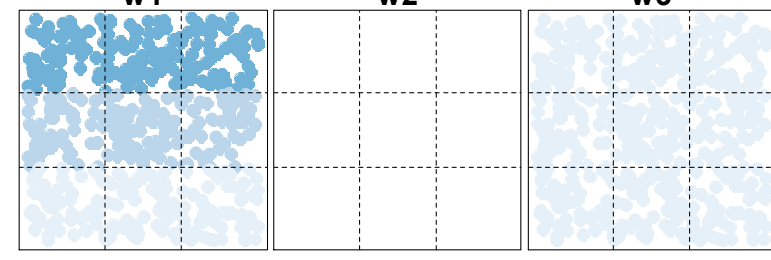

w4

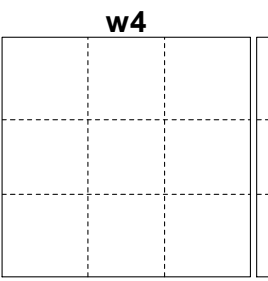

w5

w6

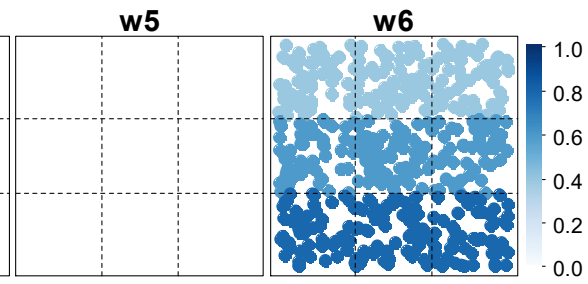

Panel 1. Simulated LWQS parameter values in scenario 2.
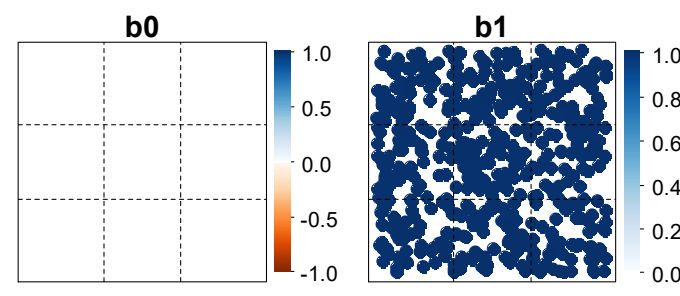

w2

w3

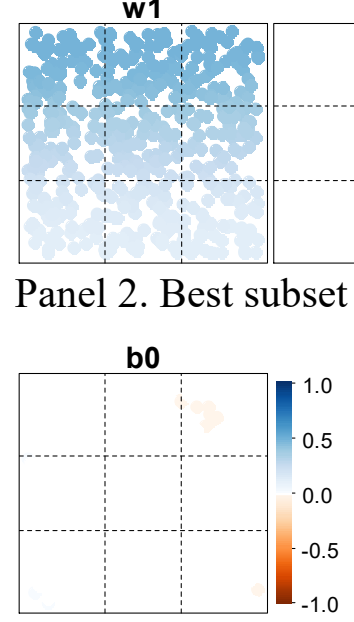

w2

w4

w5

w6
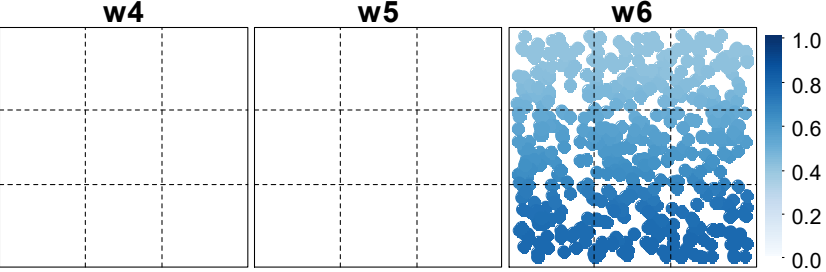

Panel 2. Best subset parameter estimates for case a) independent exposures.
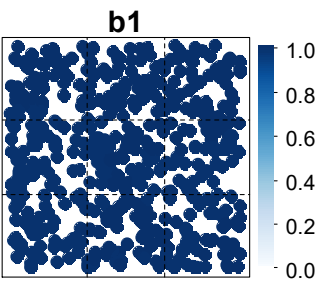

$-0.8$

$-0.6$
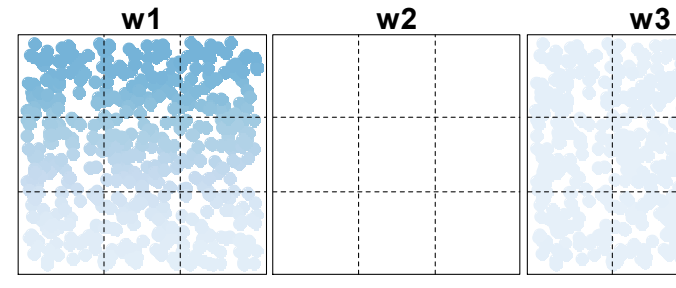

0.4
-0.2

$-0.0$

w3 w4
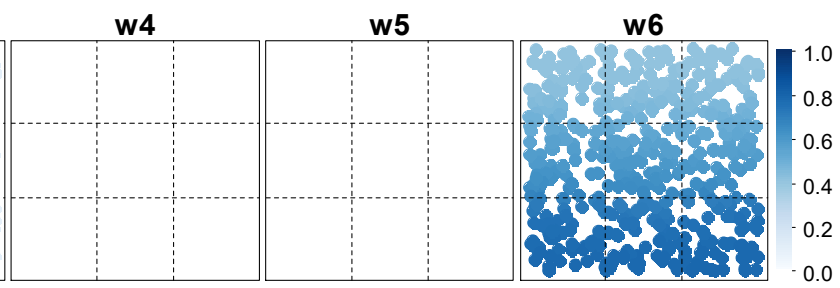

Panel 3. Best subset parameter estimates for case $b) r(x 1, x 3)=0.6$. 


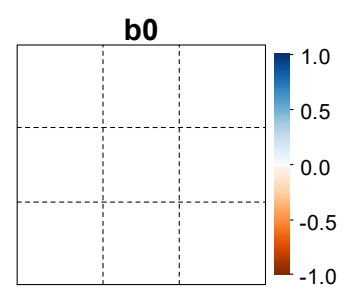

w1

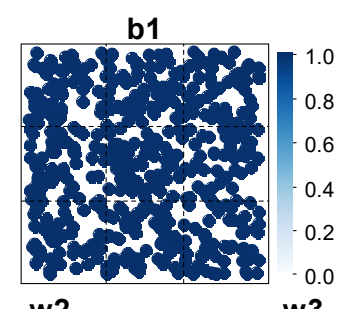
w3

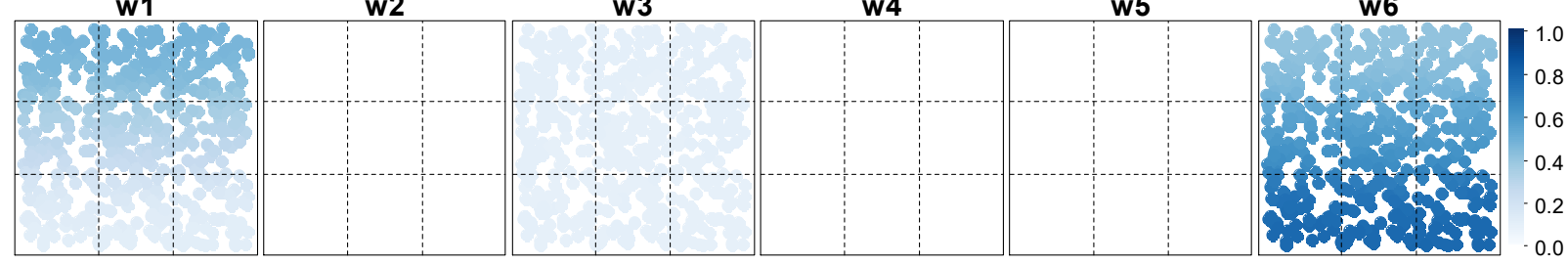

Panel 4. Best subset parameter estimates for case c) environmental correlation matrix 2.

Figure 5.11: Maps of the LWQS best subset parameter estimates in simulation scenario 2 for the cases of: a) independent exposures (panel 2); b) $r(x 1, x 3)=0.6$ (panel 3), and c) environmental correlation matrix 2 (panel 4). The simulated LWQS parameters for scenario 2 are included for the purpose of comparison (panel 1).

The parameter estimates are summarized by row of the study region for each correlation case in

Table 5.17, with error in the parameter estimates summarized similarly in Table 5.18. On

average, the best subset model correctly estimates the intercept and mixture effect with

negligible bias (Table 5.18) in all three correlation cases. When examining the estimates of the weight parameters, we see that $\mathrm{w} 3$ is on average estimated without bias in all rows of the study region for all 3 correlation cases. Additionally, w1 is generally underestimated in row 1 (its region of high activity) and overestimated in row 3 (its region of least activity). Specifically, w1 is on average, underestimated by 0.04 in row 1 and over estimated by 0.04 in row 3 , in all three correlation cases. The weight parameter for $\mathrm{x} 6$, w6, is similarly underestimated in its region of high activity, and overestimated in its region of least activity. Specifically, w6 is on average, underestimated by 0.04 in row 3 (region of high activity) and over estimated by 0.04 in row 1 (region of least activity), in all three correlation cases. The tendency of the parameter estimates to shrink toward the simulated parameter value in the middle row is evidence of the visually detected over-smoothing in Figure 5.11. Finally, we note that in the middle row of the study 
region (row 2), we see an average over estimation of 0.01 in w1 and an average underestimation of 0.01 in w6, for all correlation cases.

Table 5.17: Summary of the LWQS parameter estimates for simulation scenario 2 using the best subset ${ }^{1}$ and ensemble ${ }^{2}$ methods for the cases of a) independent exposures, b) $r(x 1, x 3)=0.6$, and c) environmental correlation structure 2 .

\begin{tabular}{|c|c|c|c|c|c|c|c|}
\hline \multirow{2}{*}{\multicolumn{2}{|c|}{ Case a }} & \multicolumn{3}{|c|}{ Best Subset Estimates } & \multicolumn{3}{|c|}{ Ensemble Estimates } \\
\hline & & Truth & Mean & Range & Truth & Mean & Range \\
\hline \multirow[t]{3}{*}{ b0 } & Row 1 & 0 & 0.00 & $(-0.01,0.01)$ & 0 & 0.05 & $(0.05,0.06)$ \\
\hline & Row 2 & 0 & 0.00 & $(-0.01,0.00)$ & 0 & 0.05 & $(0.04,0.06)$ \\
\hline & Row 3 & 0 & 0.00 & $(-0.01,0.01)$ & 0 & 0.05 & $(0.04,0.05)$ \\
\hline \multirow[t]{3}{*}{ b1 } & Row 1 & 1 & 1.00 & $(1.00,1.00)$ & 1 & 0.96 & $(0.96,0.97)$ \\
\hline & Row 2 & 1 & 1.00 & $(1.00,1.00)$ & 1 & 0.96 & $(0.96,0.97)$ \\
\hline & Row 3 & 1 & 1.00 & $(1.00,1.00)$ & 1 & 0.97 & $(0.96,0.97)$ \\
\hline \multirow[t]{3}{*}{ w1 } & Row 1 & 0.5 & 0.46 & $(0.38,0.49)$ & 0.5 & 0.41 & $(0.36,0.44)$ \\
\hline & Row 2 & 0.3 & 0.31 & $(0.20,0.38)$ & 0.3 & 0.30 & $(0.23,0.36)$ \\
\hline & Row 3 & 0.1 & 0.14 & $(0.11,0.21)$ & 0.1 & 0.18 & $(0.15,0.23)$ \\
\hline \multirow[t]{3}{*}{ w2 } & Row 1 & 0 & 0 & $(0,0)$ & 0 & 0.00 & $(0.00,0.01)$ \\
\hline & Row 2 & 0 & 0 & $(0,0)$ & 0 & 0.00 & $(0.00,0.01)$ \\
\hline & Row 3 & 0 & 0 & $(0,0)$ & 0 & 0.01 & $(0.00,0.01)$ \\
\hline \multirow[t]{3}{*}{ w3 } & Row 1 & 0.1 & 0.10 & $(0.10,0.10)$ & 0.1 & 0.09 & $(0.09,0.09)$ \\
\hline & Row 2 & 0.1 & 0.10 & $(0.10,0.10)$ & 0.1 & 0.09 & $(0.09,0.09)$ \\
\hline & Row 3 & 0.1 & 0.10 & $(0.10,0.10)$ & 0.1 & 0.09 & $(0.09,0.09)$ \\
\hline \multirow[t]{3}{*}{ w4 } & Row 1 & 0 & 0 & $(0,0)$ & 0 & 0.00 & $(0.00,0.01)$ \\
\hline & Row 2 & 0 & 0 & $(0,0)$ & 0 & 0.00 & $(0.00,0.01)$ \\
\hline & Row 3 & 0 & 0 & $(0$, & 0 & 0.01 & $(0.00,0.01)$ \\
\hline \multirow[t]{3}{*}{ w5 } & Row 1 & 0 & 0 & $(0,0)$ & 0 & 0.01 & $(0.00,0.01)$ \\
\hline & Row 2 & 0 & 0 & $(0,0)$ & 0 & 0.01 & $(0.00,0.01)$ \\
\hline & Row 3 & 0 & 0 & $(0,0)$ & 0 & 0.00 & $(0.00,0.01)$ \\
\hline \multirow[t]{3}{*}{ w6 } & Row 1 & 0.4 & 0.44 & $(0.41,0.52)$ & 0.4 & 0.49 & $(0.46,0.54)$ \\
\hline & Row 2 & 0.6 & 0.59 & $(0.52,0.70)$ & 0.6 & 0.60 & $(0.54,0.67)$ \\
\hline & Row 3 & 0.8 & 0.76 & $(0.69,0.79)$ & 0.8 & 0.72 & $(0.66,0.75)$ \\
\hline \multicolumn{8}{|c|}{ Case b } \\
\hline \multirow[t]{3}{*}{ b0 } & Row 1 & 0 & -0.01 & $(-0.01,0.01)$ & 0 & 0.03 & $(0.02,0.03)$ \\
\hline & Row 2 & 0 & 0.00 & $(-0.01,0.01)$ & 0 & 0.03 & $(0.03,0.03)$ \\
\hline & Row 3 & 0 & 0.00 & $(-0.01,0.01)$ & 0 & 0.03 & $(0.02,0.03)$ \\
\hline \multirow[t]{3}{*}{ b1 } & Row 1 & 1 & 1.00 & $(1.00,1.01)$ & 1 & 0.98 & $(0.98,0.98)$ \\
\hline & Row 2 & 1 & 1.00 & $(0.99,1.00)$ & 1 & 0.98 & $(0.98,0.98)$ \\
\hline & Row 3 & 1 & 1.00 & $(0.99,1.00)$ & 1 & 0.98 & $(0.98,0.99)$ \\
\hline \multirow[t]{3}{*}{ w1 } & Row 1 & 0.5 & 0.46 & $(0.38,0.49)$ & 0.5 & 0.41 & $(0.36,0.44)$ \\
\hline & Row 2 & 0.3 & 0.31 & $(0.20,0.39)$ & 0.3 & 0.31 & $(0.24,0.36)$ \\
\hline & Row 3 & 0.1 & 0.14 & $(0.11,0.21)$ & 0.1 & 0.19 & $(0.16,0.24)$ \\
\hline \multirow[t]{2}{*}{ w2 } & Row 1 & 0 & 0 & $(0,0)$ & 0 & 0.00 & $(0.00,0.01)$ \\
\hline & Row 2 & 0 & 0 & $(0,0)$ & 0 & 0.00 & $(0.00,0.00)$ \\
\hline
\end{tabular}




\begin{tabular}{|c|c|c|c|c|c|c|c|}
\hline & Row 3 & 0 & 0 & $(0,0)$ & 0 & 0.00 & $(0.00,0.00)$ \\
\hline \multirow[t]{3}{*}{ w3 } & Row 1 & 0.1 & 0.10 & $(0.10,0.10)$ & 0.1 & 0.10 & $(0.10,0.10)$ \\
\hline & Row 2 & 0.1 & 0.10 & $(0.10,0.10)$ & 0.1 & 0.10 & $(0.09,0.10)$ \\
\hline & Row 3 & 0.1 & 0.10 & $(0.10,0.10)$ & 0.1 & 0.09 & $(0.09,0.09)$ \\
\hline \multirow[t]{3}{*}{ w4 } & Row 1 & 0 & 0 & $(0,0)$ & 0 & 0.00 & $(0.00,0.01)$ \\
\hline & Row 2 & 0 & 0 & $(0,0)$ & 0 & 0.00 & $(0.00,0.01)$ \\
\hline & Row 3 & 0 & 0 & $(0,0)$ & 0 & 0.00 & $(0.00,0.01)$ \\
\hline \multirow[t]{3}{*}{ w5 } & Row 1 & 0 & 0 & $(0,0)$ & 0 & 0.00 & $(0.00,0.01)$ \\
\hline & Row 2 & 0 & 0 & $(0,0)$ & 0 & 0.00 & $(0.00,0.01)$ \\
\hline & Row 3 & 0 & 0 & $(0,0)$ & 0 & 0.00 & $(0.00,0.01)$ \\
\hline \multirow[t]{3}{*}{ w6 } & Row 1 & 0.4 & 0.44 & $(0.41,0.52)$ & 0.4 & 0.48 & $(0.45,0.53)$ \\
\hline & Row 2 & 0.6 & 0.59 & $(0.51,0.70)$ & 0.6 & 0.58 & $(0.53,0.65)$ \\
\hline & Row 3 & 0.8 & 0.76 & $(0.69,0.79)$ & 0.8 & 0.70 & $(0.65,0.73)$ \\
\hline \multicolumn{8}{|c|}{ Case c } \\
\hline \multirow[t]{3}{*}{ b0 } & Row 1 & 0 & 0.00 & $(0.00,0.01)$ & 0 & 0.00 & $(0.00,0.00)$ \\
\hline & Row 2 & 0 & 0.00 & $(-0.01,0.00)$ & 0 & 0.00 & $(-0.01,0.00)$ \\
\hline & Row 3 & 0 & 0.00 & $(-0.01,0.00)$ & 0 & 0.00 & $(-0.01,0.00)$ \\
\hline \multirow[t]{3}{*}{ b1 } & Row 1 & 1 & 1.00 & $(1.00,1.00)$ & 1 & 1.00 & $(1.00,1.00)$ \\
\hline & Row 2 & 1 & 1.00 & $(1.00,1.00)$ & 1 & 1.00 & $(1.00,1.00)$ \\
\hline & Row 3 & 1 & 1.00 & $(1.00,1.00)$ & 1 & 1.00 & $(1.00,1.00)$ \\
\hline \multirow[t]{3}{*}{ w1 } & Row 1 & 0.5 & 0.46 & $(0.37,0.49)$ & 0.5 & 0.40 & $(0.35,0.42)$ \\
\hline & Row 2 & 0.3 & 0.31 & $(0.20,0.38)$ & 0.3 & 0.30 & $(0.23,0.35)$ \\
\hline & Row 3 & 0.1 & 0.14 & $(0.11,0.21)$ & 0.1 & 0.19 & $(0.16,0.23)$ \\
\hline \multirow[t]{3}{*}{ w2 } & Row 1 & 0 & 0 & $(0,0)$ & 0 & 0.02 & $(0.02,0.02)$ \\
\hline & Row 2 & 0 & 0 & $(0,0)$ & 0 & 0.02 & $(0.02,0.02)$ \\
\hline & Row 3 & 0 & 0 & $(0,0)$ & 0 & 0.02 & $(0.02,0.02)$ \\
\hline \multirow[t]{3}{*}{ w3 } & Row 1 & 0.1 & 0.10 & $(0.10,0.11)$ & 0.1 & 0.09 & $(0.09,0.09)$ \\
\hline & Row 2 & 0.1 & 0.10 & $(0.10,0.11)$ & 0.1 & 0.09 & $(0.08,0.09)$ \\
\hline & Row 3 & 0.1 & 0.10 & $(0.10,0.10)$ & 0.1 & 0.08 & $(0.08,0.08)$ \\
\hline \multirow[t]{3}{*}{ w4 } & Row 1 & 0 & 0 & $(0,0)$ & 0 & 0.01 & $(0.01,0.01)$ \\
\hline & Row 2 & 0 & 0 & $(0,0)$ & 0 & 0.01 & $(0.01,0.01)$ \\
\hline & Row 3 & 0 & 0 & $(0,0)$ & 0 & 0.01 & $(0.00,0.01)$ \\
\hline \multirow[t]{3}{*}{ w5 } & Row 1 & 0 & 0 & $(0,0)$ & 0 & 0.02 & $(0.02,0.02)$ \\
\hline & Row 2 & 0 & 0 & $(0,0)$ & 0 & 0.02 & $(0.02,0.02)$ \\
\hline & Row 3 & 0 & 0 & $(0,0)$ & 0 & 0.02 & $(0.02,0.02)$ \\
\hline \multirow[t]{3}{*}{ w6 } & Row 1 & 0.4 & 0.44 & $(0.41,0.52)$ & 0.4 & 0.47 & $(0.45,0.52)$ \\
\hline & Row 2 & 0.6 & 0.59 & $(0.51,0.70)$ & 0.6 & 0.58 & $(0.52,0.64)$ \\
\hline & Row 3 & 0.8 & 0.76 & $(0.69,0.79)$ & 0.8 & 0.69 & $(0.64,0.72)$ \\
\hline
\end{tabular}

\footnotetext{
${ }^{1}$ Best subset estimates pertain to $\mathrm{S}_{\mathrm{b}}=\{\mathrm{x} 1, \mathrm{x} 3, \mathrm{x} 6\}$ with estimated bandwidth value $\gamma=0.1$.

${ }^{2}$ Ensemble estimates are calculated using subsets of size 5.
} 
Table 5.18: Summary of the bias and RMSE in the estimated LWQS parameters for simulation scenario 2 using the best subset ${ }^{1}$ and ensemble ${ }^{2}$ methods for the cases of a) independent exposures, $b) r(x 1, x 3)=0.6, c)$ environmental correlation structure 2 .

Best Subset Estimates

Ensemble Estimates

\begin{tabular}{|c|c|c|c|c|c|c|c|}
\hline \multirow{2}{*}{\multicolumn{2}{|c|}{ Case a }} & \multirow{3}{*}{$\begin{array}{r}\text { Avg. Bias } \\
0.00\end{array}$} & \multirow{3}{*}{$\begin{array}{r}\text { Bias Range } \\
(-0.01,0.01)\end{array}$} & \multirow{3}{*}{$\begin{array}{r}\text { RMSE } \\
0.00\end{array}$} & \multirow{3}{*}{$\begin{array}{r}\text { Avg. Bias } \\
0.05\end{array}$} & \multirow{3}{*}{$\frac{\text { Bias Range }}{(0.05,0.06)}$} & \multirow{3}{*}{$\begin{array}{r}\text { RMSE } \\
0.05\end{array}$} \\
\hline & & & & & & & \\
\hline b0 & Row 1 & & & & & & \\
\hline & Row 2 & 0.00 & $(-0.01,0.00)$ & 0.00 & 0.05 & $(0.04,0.06)$ & 0.05 \\
\hline & Row 3 & 0.00 & $(-0.01,0.01)$ & 0.00 & 0.05 & $(0.04,0.05)$ & 0.05 \\
\hline \multirow[t]{3}{*}{ b1 } & Row 1 & 0.00 & $(0.00,0.00)$ & 0.00 & -0.04 & $(-0.04,-0.03)$ & 0.04 \\
\hline & Row 2 & 0.00 & $(0.00,0.00)$ & 0.00 & -0.04 & $(-0.04,-0.03)$ & 0.04 \\
\hline & Row 3 & 0.00 & $(0.00,0.00)$ & 0.00 & -0.03 & $(-0.04,-0.03)$ & 0.03 \\
\hline \multirow[t]{3}{*}{ w1 } & Row 1 & -0.04 & $(-0.12,-0.01)$ & 0.05 & -0.09 & $(-0.14,-0.06)$ & 0.09 \\
\hline & Row 2 & 0.01 & $(-0.10,0.08)$ & 0.05 & 0.00 & $(-0.07,0.06)$ & 0.03 \\
\hline & Row 3 & 0.04 & $(0.01,0.11)$ & 0.05 & 0.08 & $(0.05,0.13)$ & 0.09 \\
\hline \multirow[t]{3}{*}{ w2 } & Row 1 & 0 & $(0,0)$ & 0 & 0.00 & $(0.00,0.01)$ & 0.00 \\
\hline & Row 2 & 0 & $(0,0)$ & 0 & 0.00 & $(0.00,0.01)$ & 0.00 \\
\hline & Row 3 & 0 & $(0,0)$ & 0 & 0.01 & $(0.00,0.01)$ & 0.01 \\
\hline \multirow[t]{3}{*}{ w3 } & Row 1 & 0.00 & $(0.00,0.00)$ & 0.00 & -0.01 & $(-0.01,-0.01)$ & 0.01 \\
\hline & Row 2 & 0.00 & $(0.00,0.00)$ & 0.00 & -0.01 & $(-0.01,-0.01)$ & 0.01 \\
\hline & Row 3 & 0.00 & $(0.00,0.00)$ & 0.00 & -0.01 & $(-0.01,-0.01)$ & 0.01 \\
\hline \multirow[t]{3}{*}{ w4 } & Row 1 & 0 & $(0,0)$ & 0 & 0.00 & $(0.00,0.01)$ & 0.00 \\
\hline & Row 2 & 0 & $(0,0)$ & 0 & 0.00 & $(0.00,0.01)$ & 0.00 \\
\hline & Row 3 & 0 & $(0,0)$ & 0 & 0.01 & $(0.00,0.01)$ & 0.01 \\
\hline \multirow[t]{3}{*}{ w5 } & Row 1 & 0 & $(0,0)$ & 0 & 0.01 & $(0.00,0.01)$ & 0.01 \\
\hline & Row 2 & 0 & $(0,0)$ & 0 & 0.01 & $(0.00,0.01)$ & 0.01 \\
\hline & Row 3 & 0 & $(0,0)$ & 0 & 0.00 & $(0.00,0.01)$ & 0.00 \\
\hline \multirow[t]{3}{*}{ w6 } & Row 1 & 0.04 & $(0.01,0.12)$ & 0.05 & 0.09 & $(0.06,0.14)$ & 0.09 \\
\hline & Row 2 & -0.01 & $(-0.08,0.10)$ & 0.05 & 0.00 & $(-0.06,0.07)$ & 0.03 \\
\hline & Row 3 & -0.04 & $(-0.11,-0.01)$ & 0.05 & -0.08 & $(-0.14,-0.05)$ & 0.09 \\
\hline \multicolumn{8}{|c|}{ Case b } \\
\hline \multirow[t]{3}{*}{ b0 } & Row 1 & -0.01 & $(-0.01,0.01)$ & 0.01 & 0.03 & $(0.02,0.03)$ & 0.03 \\
\hline & Row 2 & 0.00 & $(-0.01,0.01)$ & 0.00 & 0.03 & $(0.03,0.03)$ & 0.03 \\
\hline & Row 3 & 0.00 & $(-0.01,0.01)$ & 0.00 & 0.03 & $(0.02,0.03)$ & 0.03 \\
\hline \multirow[t]{3}{*}{ b1 } & Row 1 & 0.00 & $(0.00,0.01)$ & 0.00 & -0.02 & $(-0.02,-0.02)$ & 0.02 \\
\hline & Row 2 & 0.00 & $(-0.01,0.00)$ & 0.00 & -0.02 & $(-0.02,-0.02)$ & 0.02 \\
\hline & Row 3 & 0.00 & $(-0.01,0.00)$ & 0.00 & -0.02 & $(-0.02,-0.01)$ & 0.02 \\
\hline \multirow[t]{3}{*}{ w1 } & Row 1 & -0.04 & $(-0.12,-0.01)$ & 0.05 & -0.09 & $(-0.14,-0.06)$ & 0.09 \\
\hline & Row 2 & 0.01 & $(-0.10,0.09)$ & 0.04 & 0.01 & $(-0.06,0.06)$ & 0.03 \\
\hline & Row 3 & 0.04 & $(0.01,0.11)$ & 0.05 & 0.09 & $(0.06,0.14)$ & 0.10 \\
\hline \multirow[t]{3}{*}{ w2 } & Row 1 & 0 & $(0,0)$ & 0 & 0.00 & $(0.00,0.01)$ & 0.00 \\
\hline & Row 2 & 0 & $(0,0)$ & 0 & 0.00 & $(0.00,0.00)$ & 0.00 \\
\hline & Row 3 & 0 & $(0,0)$ & 0 & 0.00 & $(0.00,0.00)$ & 0.00 \\
\hline \multirow[t]{3}{*}{ w3 } & Row 1 & 0.00 & $(0.00,0.00)$ & 0.00 & 0.00 & $(0.00,0.00)$ & 0.00 \\
\hline & Row 2 & 0.00 & $(0.00,0.00)$ & 0.00 & 0.00 & $(-0.01,0.00)$ & 0.00 \\
\hline & Row 3 & 0.00 & $(0.00,0.00)$ & 0.00 & -0.01 & $(-0.01,-0.01)$ & 0.01 \\
\hline w4 & Row 1 & 0 & $(0,0)$ & 0 & 0.00 & $(0.00,0.01)$ & 0.00 \\
\hline
\end{tabular}




\begin{tabular}{|c|c|c|c|c|c|c|c|}
\hline & Row 2 & 0 & $(0,0)$ & 0 & 0.00 & $(0.00,0.01)$ & 0.00 \\
\hline & Row 3 & 0 & $(0,0)$ & 0 & 0.00 & $(0.00,0.01)$ & 0.00 \\
\hline \multirow[t]{3}{*}{ w5 } & Row 1 & 0 & $(0,0)$ & 0 & 0.00 & $(0.00,0.01)$ & 0.00 \\
\hline & Row 2 & 0 & $(0,0)$ & 0 & 0.00 & $(0.00,0.01)$ & 0.00 \\
\hline & Row 3 & 0 & $(0,0)$ & 0 & 0.00 & $(0.00,0.01)$ & 0.00 \\
\hline \multirow[t]{3}{*}{ w6 } & Row 1 & 0.04 & $(0.01,0.12)$ & 0.05 & 0.08 & $(0.05,0.13)$ & 0.08 \\
\hline & Row 2 & -0.01 & $(-0.09,0.10)$ & 0.05 & -0.02 & $(-0.07,0.05)$ & 0.04 \\
\hline & Row 3 & -0.04 & $(-0.11,-0.01)$ & 0.05 & -0.10 & $(-0.15,-0.07)$ & 0.10 \\
\hline \multicolumn{8}{|c|}{ Case c } \\
\hline \multirow[t]{3}{*}{ b0 } & Row 1 & 0.00 & $(0.00,0.01)$ & 0.00 & 0.00 & $(0.00,0.00)$ & 0.00 \\
\hline & Row 2 & 0.00 & $(-0.01,0.00)$ & 0.00 & 0.00 & $(-0.01,0.00)$ & 0.00 \\
\hline & Row 3 & 0.00 & $(-0.01,0.00)$ & 0.00 & 0.00 & $(-0.01,0.00)$ & 0.00 \\
\hline \multirow[t]{3}{*}{ b1 } & Row 1 & 0.00 & $(0.00,0.00)$ & 0.00 & 0.00 & $(0.00,0.00)$ & 0.00 \\
\hline & Row 2 & 0.00 & $(0.00,0.00)$ & 0.00 & 0.00 & $(0.00,0.00)$ & 0.00 \\
\hline & Row 3 & 0.00 & $(0.00,0.00)$ & 0.00 & 0.00 & $(0.00,0.00)$ & 0.00 \\
\hline \multirow[t]{3}{*}{ w1 } & Row 1 & -0.04 & $(-0.13,-0.01)$ & 0.05 & -0.10 & $(-0.15,-0.08)$ & 0.10 \\
\hline & Row 2 & 0.01 & $(-0.10,0.08)$ & 0.04 & 0.00 & $(-0.07,0.05)$ & 0.03 \\
\hline & Row 3 & 0.04 & $(0.01,0.11)$ & 0.05 & 0.09 & $(0.06,0.13)$ & 0.09 \\
\hline \multirow[t]{3}{*}{ w2 } & Row 1 & 0 & $(0,0)$ & 0 & 0.02 & $(0.02,0.02)$ & 0.02 \\
\hline & Row 2 & 0 & $(0,0)$ & 0 & 0.02 & $(0.02,0.02)$ & 0.02 \\
\hline & Row 3 & 0 & $(0,0)$ & 0 & 0.02 & $(0.02,0.02)$ & 0.02 \\
\hline \multirow[t]{3}{*}{ w3 } & Row 1 & 0.00 & $(0.00,0.01)$ & 0.00 & -0.01 & $(-0.01,-0.01)$ & 0.01 \\
\hline & Row 2 & 0.00 & $(0.00,0.01)$ & 0.00 & -0.01 & $(-0.02,-0.01)$ & 0.01 \\
\hline & Row 3 & 0.00 & $(0.00,0.00)$ & 0.00 & -0.02 & $(-0.02,-0.02)$ & 0.02 \\
\hline \multirow[t]{3}{*}{ w4 } & Row 1 & 0 & $(0,0)$ & 0 & 0.01 & $(0.01,0.01)$ & 0.01 \\
\hline & Row 2 & 0 & $(0,0)$ & 0 & 0.01 & $(0.01,0.01)$ & 0.01 \\
\hline & Row 3 & 0 & $(0,0)$ & 0 & 0.01 & $(0.00,0.01)$ & 0.01 \\
\hline \multirow[t]{3}{*}{ w5 } & Row 1 & 0 & $(0,0)$ & 0 & 0.02 & $(0.02,0.02)$ & 0.02 \\
\hline & Row 2 & 0 & $(0,0)$ & 0 & 0.02 & $(0.02,0.02)$ & 0.02 \\
\hline & Row 3 & 0 & $(0,0)$ & 0 & 0.02 & $(0.02,0.02)$ & 0.02 \\
\hline \multirow[t]{3}{*}{ w6 } & Row 1 & 0.04 & $(0.01,0.12)$ & 0.05 & 0.07 & $(0.05,0.12)$ & 0.08 \\
\hline & Row 2 & -0.01 & $(-0.09,0.10)$ & 0.04 & -0.02 & $(-0.08,0.04)$ & 0.04 \\
\hline & Row 3 & -0.04 & $(-0.11,-0.01)$ & 0.05 & -0.11 & $(-0.16,-0.08)$ & 0.11 \\
\hline
\end{tabular}

${ }^{1}$ Best subset estimates pertain to $\mathrm{S}_{\mathrm{b}}=\{\mathrm{x} 1, \mathrm{x} 3, \mathrm{x} 6\}$ with estimated bandwidth value $\gamma=0.1$.

${ }^{2}$ Ensemble estimates are calculated using subsets of size 5.

The average bias in the best subset estimates is mapped over the study region in Figure 5.12.

The overestimation of $w 1$ in row 3 and w6 in row 1 is visualized in grey. Conversely, the underestimation of $\mathrm{w} 1$ in row 1 and w6 in row 3 is visualized in red. The reversal in the direction of bias near the linear boundaries (row divisions) is further evidence of over smoothing. 


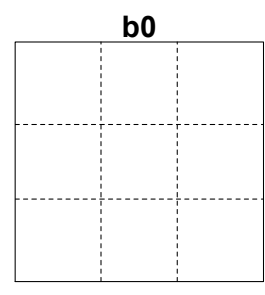

w1

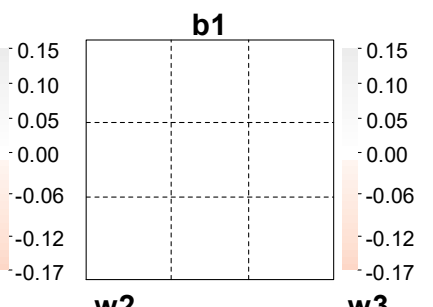

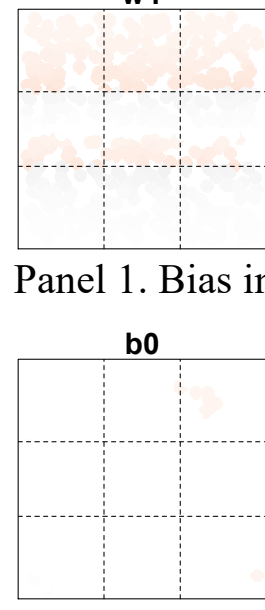

w1

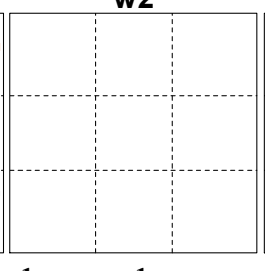

w3

w4

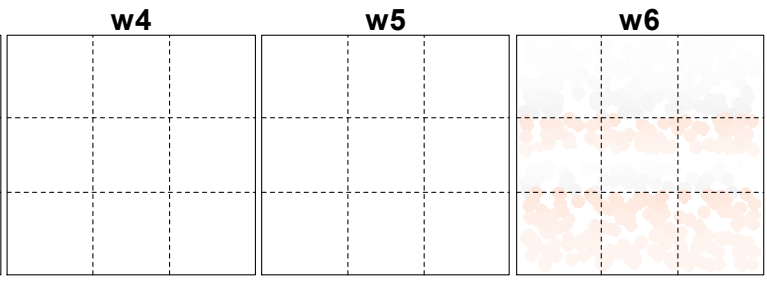
-0.15
-0.10
-0.05
-0.00
-0.06
-0.12
-0.17
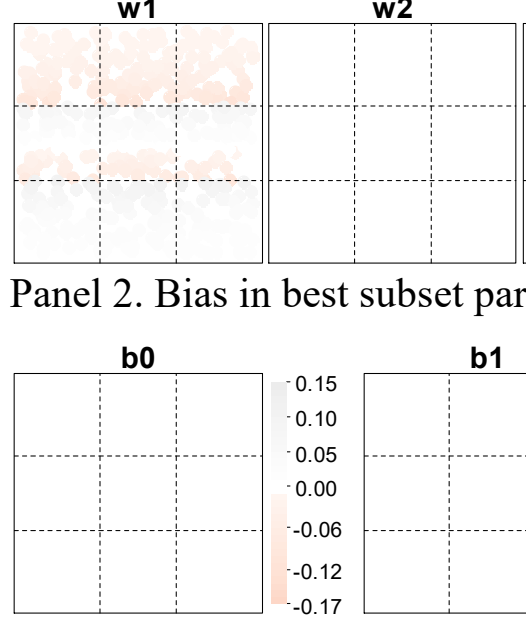

b1

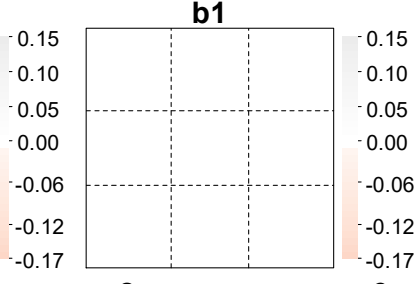

w3
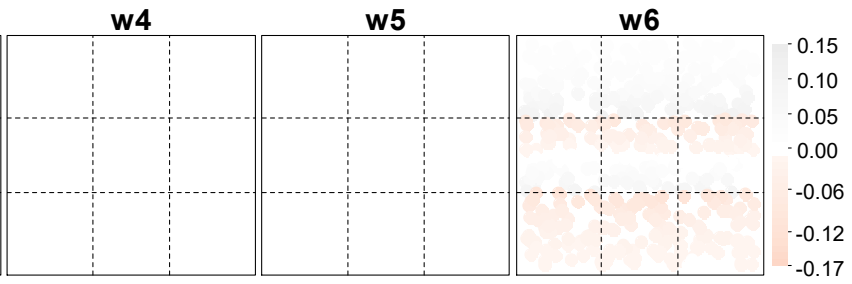

w1

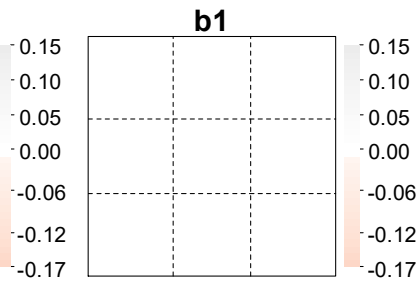

w2 w3
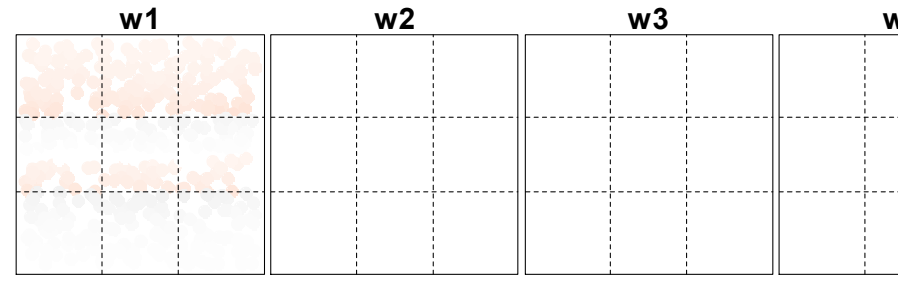

w4
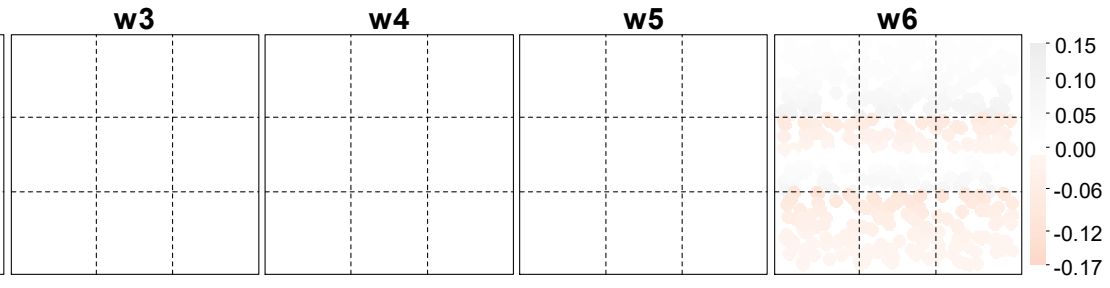

Panel 3. Bias in best subset parameter estimates foe 5 for c) environmental correlation matrix 2.

Figure 5.12: Bias in the best subset parameter estimates for scenario 2 in the cases of a) independent exposures (panel 1), b) $\mathrm{r}(\mathrm{x} 1, \mathrm{x} 3)=0.6$ (panel 2), and c) environmental correlation structure 2 (panel 3). 
Maps of the ensemble estimates are provided in Figure 5.13, where we note overestimation of the intercept, $\mathrm{b} 0$, and underestimation of the mixture effect, $\mathrm{b} 1$, in the first two correlation cases. In contrast, the ensemble method estimates both the intercept and mixture effect with negligible error in the third, most complicated correlation case. Table 5.18, demonstrates that the magnitude of the bias in $\mathrm{b} 0$ and $\mathrm{b} 1$ decreases with increasing complexity in the correlation among exposures. More specifically, the magnitude of bias associated with $\mathrm{b} 0$ and $\mathrm{b} 1$ is greatest in the case of independent exposures ( $\mathrm{bias}=0.05$ and -0.04 for $\mathrm{b} 0$ and $\mathrm{b} 1$, respectively) and effectively zero in the most complex correlation case, i.e., environmental correlation matrix 2 . The trend in bias for b0 and b1 is visually apparent in Figure 5.14, where the bias in the ensemble estimates is mapped over the study area for each correlation case.
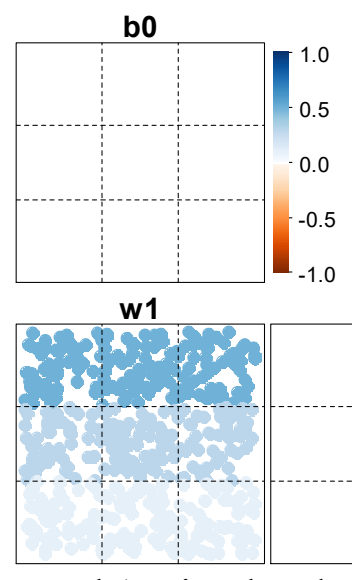

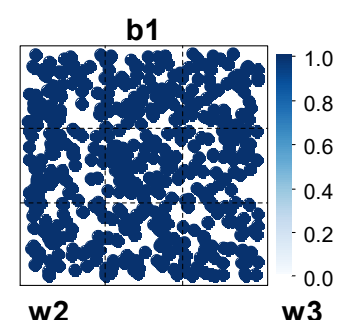

w2 w3

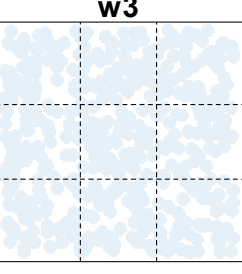

w4

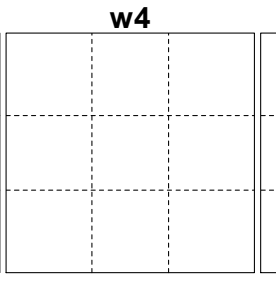

w5

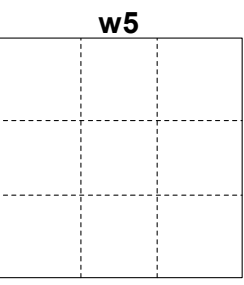

w6

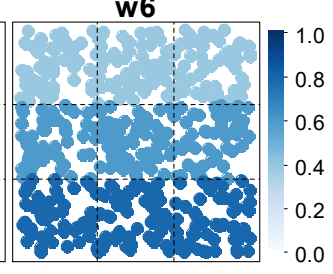

Panel 1. Simulated LWQS parameter values in scenario 2. 


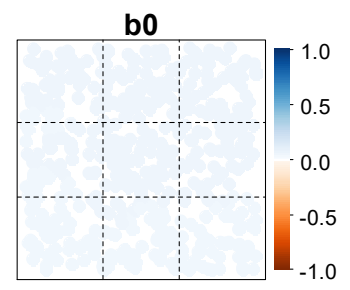

w1

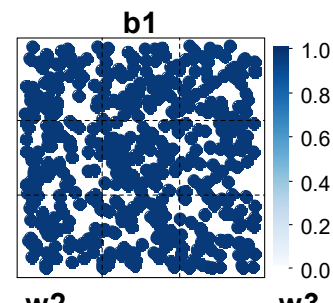

w3

w4

w5 w6

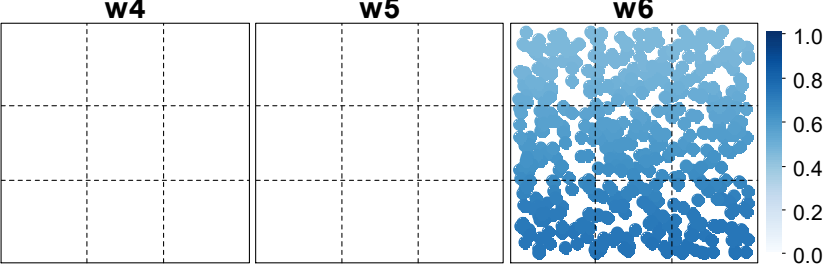

Panel 2. Ensemble estimates from subsets of size 5 for case a) independent exposures.

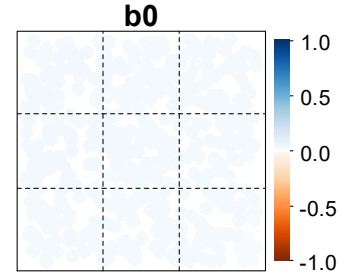

w1

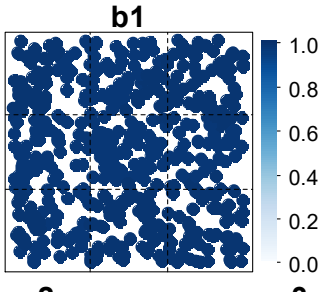

w2 w3

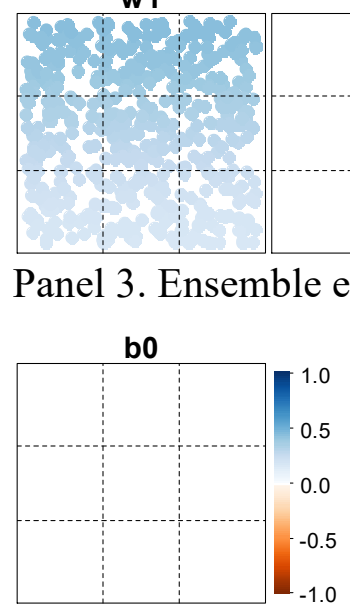

w1

w2

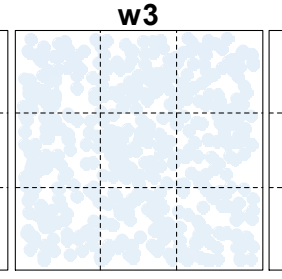

w4

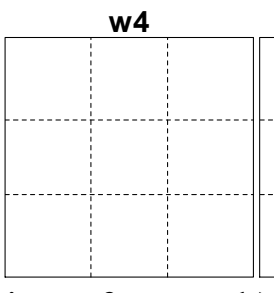

w5

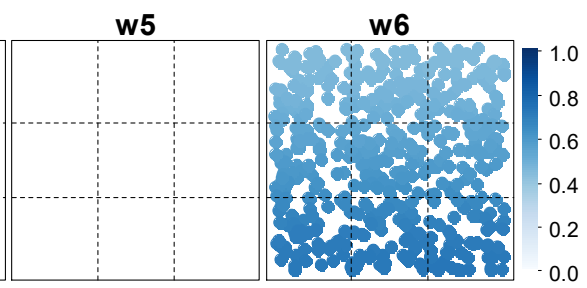

b) $r(x 1, x 3)=0.6$
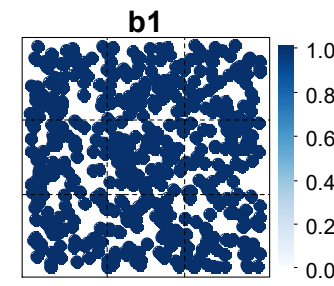

w2 w3
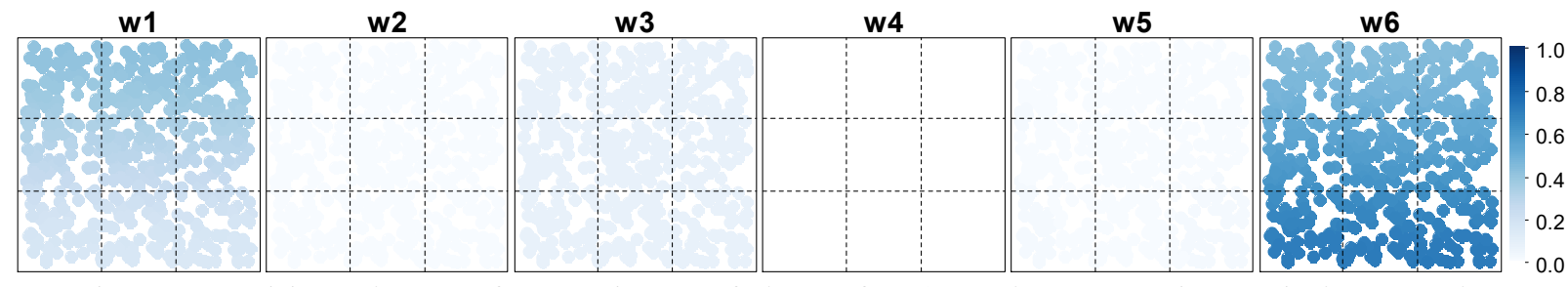

Panel 4. Ensemble estimates from subsets of size 5 for c) environmental correlation matrix 2.

Figure 5.13: Maps of the LWQS ensemble parameter estimates from subsets of size 5 for scenario 2 in the cases of a) independent exposures (panel 2), b) r(x1,x3) =0.6 (panel 3), and c) environmental correlation matrix 2 (panel 4). The scenario 2 simulated LWQS parameters (panel 1) are included for the purpose of comparison. 


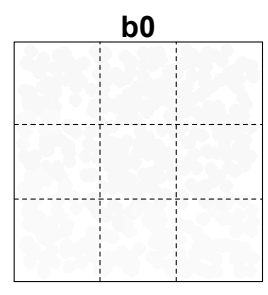

w1

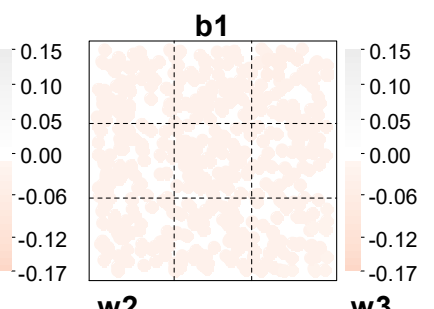

w3

w4

w5

w6

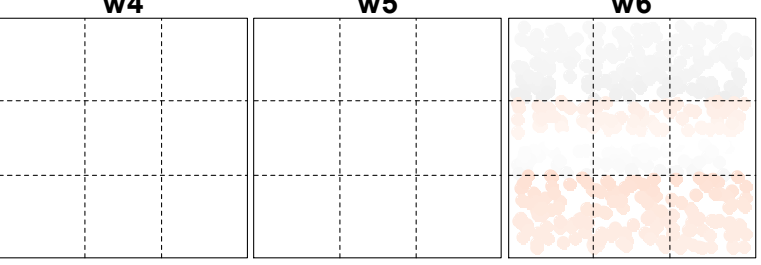

-0.15
-0.10
-0.05
-0.00
-0.06
-0.12
-0.17

Panel 1. Bias in ensemble parameter estimates from subsets of size 5 for a) independent exposures.
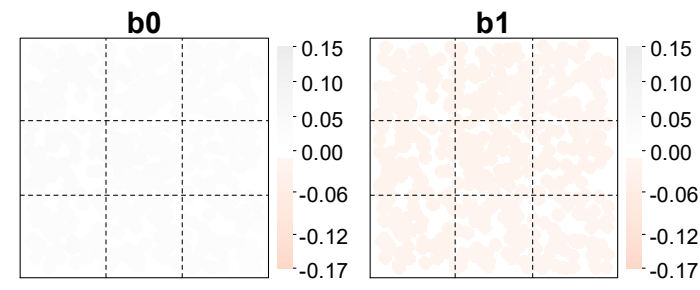

$\mathbf{w 1}$ w2 w3
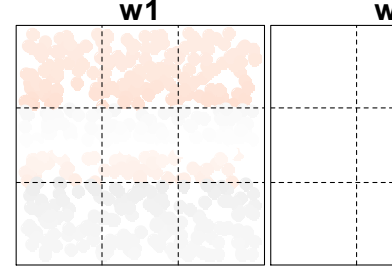

2

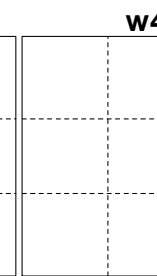

w4
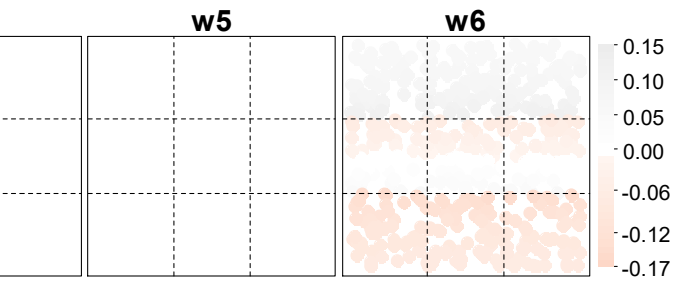

Panel 2. Bias in ensemble parameter estimates from subsets of size 5 for $b) r(x 1, x 3)=0.6$.
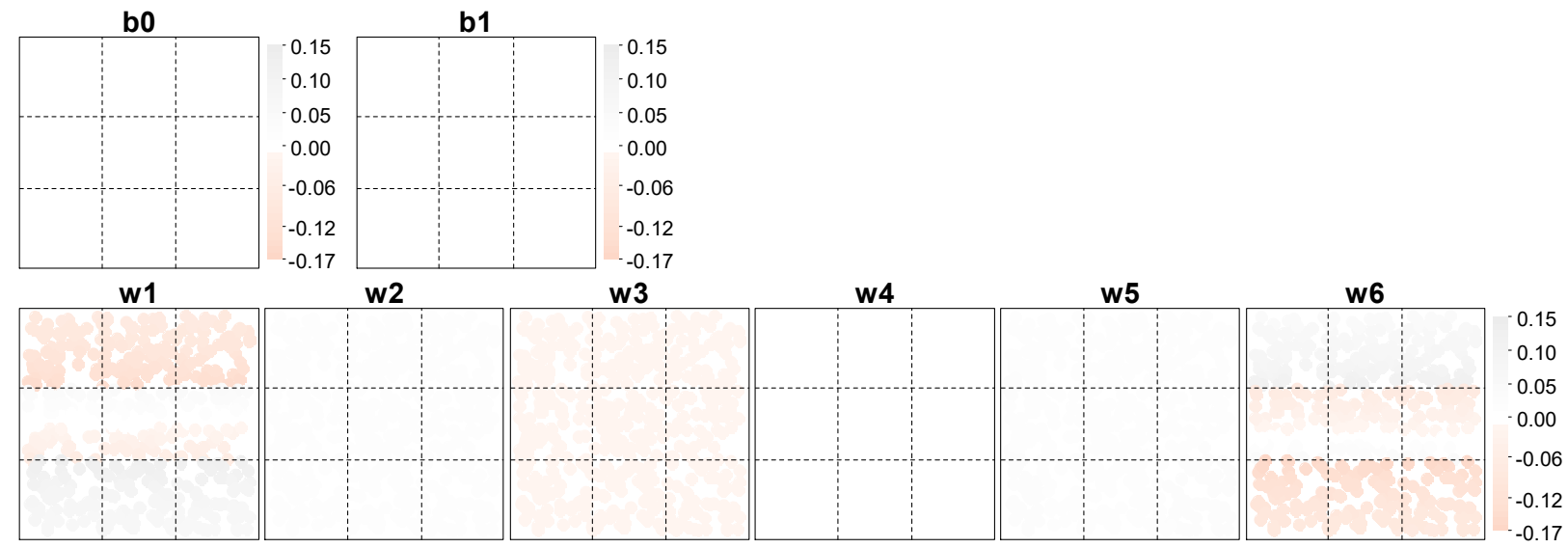

Panel 3. Bias in ensemble parameter estimates from subsets of size 5 for c) environmental correlation matrix 2 .

Figure 5.14: Bias in ensemble parameter estimates from subsets of size 5 for scenario 2 in the cases of a) independent exposures (panel 1), b) $\mathrm{r}(\mathrm{x} 1, \mathrm{x} 3)=0.6$ (panel 2), and c) environmental correlation structure 2 (panel 3). 
As shown in Figures 5.13 and 5.14, the ensemble estimates of the weight parameters demonstrate a more exaggerated version of the over smoothing seen in the best subset estimates. The exaggerated over-smoothing is likely attributable to the averaging of solutions from subset models with larger estimated bandwidth parameters resulting from the inclusion of unimportant exposures and/or the exclusion of important exposures with spatially-dependent effects.

Finally, we note that although the ensemble method produces non-zero estimates of w2, w4, and w5, in the most highly correlated case, none of these exposures are selected as important when using the pre-specified cut-off of 0.05 . More generally, when using a cut-off of 0.05 , it can be stated that the ensemble estimates correctly select important exposures and correctly exclude unimportant exposures, in all correlation cases.

In scenario 2 we have shown that the LWQS method is able to detect the general pattern in spatially dependent weight parameters and accurately estimate the mixture effect even in the presence of a complex environmental correlation pattern among exposures. Both the best subset and ensemble estimates demonstrate the ability to correctly distinguish between important exposures and those unrelated to the outcome (i.e., sensitivity and specificity). While both sets of estimates had difficulty detecting the strictly linear boundaries in the spatially dependent weight parameters, the best subset method produced superior estimates - suffering from over-smoothing to a lesser extent than the ensemble estimates, in all correlation cases.

\section{Scenario 3}

Scenario 3 extends the simulation settings of scenario 2 to include spatial dependency in the mixture effect, and an environmental correlation structure of increased complexity. The simulated weight parameters in scenario 3 are the same as those in scenario 2, with w1 and w3 changing by row of the study region. Scenario 3 differs from the former scenario in that the 
mixture effect, b1, is now also set to vary by row of the study region. The simulated parameter values are listed in Table 5.19.

Table 5.19: Simulated LWQS parameters for scenario 3.

\begin{tabular}{lrrrrrrrr} 
& b0 & b1 & w1 & w2 & w3 & w4 & w5 & w6 \\
\hline Row 1 & 0.0 & 0.5 & 0.5 & 0.0 & 0.1 & 0.0 & 0.0 & 0.4 \\
Row 2 & 0.0 & 0.7 & 0.3 & 0.0 & 0.1 & 0.0 & 0.0 & 0.6 \\
Row 3 & 0.0 & 1.0 & 0.1 & 0.0 & 0.1 & 0.0 & 0.0 & 0.8 \\
\hline
\end{tabular}

As in scenario 1 and 2, the exposure data is again simulated as multivariate normal with means that are fixed over the study region. Scenario 3, however, considers two environmentally relevant correlation patterns of increasing complexity: a) environmental correlation matrix 2 (Table 5.3) in which correlations range from 0.08 to 0.91 , and b) environmental correlation matrix 3 (Table 5.4), in which correlations range from 0.87 to 0.95 . Results of scenario 1 and 2 suggest that model performance is largely unhindered in the presence of complex correlation patterns. We therefore aim to further challenge the model in scenario 3 with the inclusion of the latter correlation structure.

Model AICc and choice of bandwidth parameter are summarized for all subsets of sizes 3, 4, and 5 in Tables 5.20 and $\mathbf{5 . 2 1}$ for correlation cases a and b, respectively. We see that in both correlation cases, the best subset (i.e., model with lowest AICc) is correctly identified as the subset containing only the important exposures of $x 1, x 3$, and $x 6$. Thus, the best subset method was able to accurately perform variable selection in terms of both sensitivity and specificity for both correlation cases. Further, while subset composition is still partially reflected in the estimated bandwidth parameters, the added layer of spatial dependency (through b1) is evident, with all subsets demonstrating a tendency to choose smaller candidate bandwidth values. In 
particular, we note that the estimated bandwidth parameters do not exceed the second smallest candidate value $(\gamma=0.2)$ in the latter, most complex, correlation case (see Table 5.21).

Table 5.20: Summary of model AICc by variable subset and the corresponding number of times each candidate bandwidth value $\gamma$ was chosen over the 100 simulated data sets for scenario 3 in case a) environmental correlation structure 2 .

\begin{tabular}{|c|c|c|c|c|c|c|c|c|c|}
\hline \multirow[b]{3}{*}{ Subset } & \multirow[b]{2}{*}{ AICc } & \multicolumn{8}{|c|}{ \# Times Selected } \\
\hline & & \multicolumn{8}{|c|}{ Bandwidth Value $\gamma$} \\
\hline & Mean (SD) & 0.1 & 0.2 & 0.3 & 0.5 & 0.6 & 0.8 & 1.0 & 1.4 \\
\hline$x 1, x 2, x 3$ & $975(20.4)$ & 0 & 1 & 80 & 19 & 0 & 0 & 0 & 0 \\
\hline $\mathrm{x} 1, \mathrm{x} 2, \mathrm{x} 4$ & $975(19.3)$ & 0 & 0 & 89 & 11 & 0 & 0 & 0 & 0 \\
\hline$x 1, x 2, x 5$ & $520(40.5)$ & 0 & 100 & 0 & 0 & 0 & 0 & 0 & 0 \\
\hline$x 1, x 2, x 6$ & $-181(33.4)$ & 100 & 0 & 0 & 0 & 0 & 0 & 0 & 0 \\
\hline$x 1, x 3, x 4$ & $969(20.0)$ & 0 & 1 & 91 & 8 & 0 & 0 & 0 & 0 \\
\hline$x 1, x 3, x 5$ & $504(40.0)$ & 0 & 100 & 0 & 0 & 0 & 0 & 0 & 0 \\
\hline$x 1, x 3, x 6$ & $-230(36.4)$ & 100 & 0 & 0 & 0 & 0 & 0 & 0 & 0 \\
\hline$x 1, x 4, x 5$ & $517(40.5)$ & 0 & 100 & 0 & 0 & 0 & 0 & 0 & 0 \\
\hline$x 1, x 4, x 6$ & $-145(36.2)$ & 100 & 0 & 0 & 0 & 0 & 0 & 0 & 0 \\
\hline$x 1, x 5, x 6$ & $-143(35.8)$ & 100 & 0 & 0 & 0 & 0 & 0 & 0 & 0 \\
\hline$x 2, x 3, x 4$ & 1039 (21.4) & 0 & 0 & 78 & 22 & 0 & 0 & 0 & 0 \\
\hline$x 2, x 3, x 5$ & 604 (36.7) & 0 & 100 & 0 & 0 & 0 & 0 & 0 & 0 \\
\hline$x 2, x 3, x 6$ & $102(32.1)$ & 100 & 0 & 0 & 0 & 0 & 0 & 0 & 0 \\
\hline$x 2, x 4, x 5$ & $642(36.9)$ & 0 & 100 & 0 & 0 & 0 & 0 & 0 & 0 \\
\hline$x 2, x 4, x 6$ & $214(29.0)$ & 91 & 9 & 0 & 0 & 0 & 0 & 0 & 0 \\
\hline$x 2, x 5, x 6$ & $238(29.2)$ & 55 & 45 & 0 & 0 & 0 & 0 & 0 & 0 \\
\hline $\mathrm{x} 3, \mathrm{x} 4, \mathrm{x} 5$ & $593(36.1)$ & 0 & 100 & 0 & 0 & 0 & 0 & 0 & 0 \\
\hline$x 3, x 4, x 6$ & $112(29.7)$ & 100 & 0 & 0 & 0 & 0 & 0 & 0 & 0 \\
\hline$x 3, x 5, x 6$ & $127(32.0)$ & 100 & 0 & 0 & 0 & 0 & 0 & 0 & 0 \\
\hline$x 4, x 5, x 6$ & $399(22.5)$ & 0 & 100 & 0 & 0 & 0 & 0 & 0 & 0 \\
\hline$x 1, x 2, x 3, x 4$ & $983(20.0)$ & 0 & 0 & 48 & 51 & 1 & 0 & 0 & 0 \\
\hline $\mathrm{x} 1, \mathrm{x} 2, \mathrm{x} 3, \mathrm{x} 5$ & $529(40.5)$ & 0 & 100 & 0 & 0 & 0 & 0 & 0 & 0 \\
\hline$x 1, x 2, x 3, x 6$ & $-164(36.4)$ & 100 & 0 & 0 & 0 & 0 & 0 & 0 & 0 \\
\hline$x 1, x 2, x 4, x 5$ & $533(40.8)$ & 0 & 100 & 0 & 0 & 0 & 0 & 0 & 0 \\
\hline$x 1, x 2, x 4, x 6$ & $-114(34.7)$ & 100 & 0 & 0 & 0 & 0 & 0 & 0 & 0 \\
\hline$x 1, x 2, x 5, x 6$ & $-113(34.1)$ & 100 & 0 & 0 & 0 & 0 & 0 & 0 & 0 \\
\hline$x 1, x 3, x 4, x 5$ & $518(40.3)$ & 0 & 100 & 0 & 0 & 0 & 0 & 0 & 0 \\
\hline$x 1, x 3, x 4, x 6$ & $-161(37.1)$ & 100 & 0 & 0 & 0 & 0 & 0 & 0 & 0 \\
\hline$x 1, x 3, x 5, x 6$ & $-163(37.5)$ & 100 & 0 & 0 & 0 & 0 & 0 & 0 & 0 \\
\hline$x 1, x 4, x 5, x 6$ & $-78(37.2)$ & 100 & 0 & 0 & 0 & 0 & 0 & 0 & 0 \\
\hline$x 2, x 3, x 4, x 5$ & $610(36.8)$ & 0 & 100 & 0 & 0 & 0 & 0 & 0 & 0 \\
\hline
\end{tabular}




\begin{tabular}{llrrrrrrrr} 
x2, x3, x4, x6 & $151(29.5)$ & 81 & 19 & 0 & 0 & 0 & 0 & 0 & 0 \\
x2, x3, x5, x6 & $163(29.9)$ & 63 & 37 & 0 & 0 & 0 & 0 & 0 & 0 \\
x2, x4, x5, x6 & $250(25.8)$ & 3 & 97 & 0 & 0 & 0 & 0 & 0 & 0 \\
x3, x4, x5, x6 & $170(28.0)$ & 39 & 61 & 0 & 0 & 0 & 0 & 0 & 0 \\
& & & & & & & & & \\
x1, x2, x3, x4, x5 & $542(40.7)$ & 0 & 100 & 0 & 0 & 0 & 0 & 0 & 0 \\
x1, x2, x3, x4, x6 & $-94(37.1)$ & 100 & 0 & 0 & 0 & 0 & 0 & 0 & 0 \\
x1, x2, x3, x5, x6 & $-96(37.2)$ & 100 & 0 & 0 & 0 & 0 & 0 & 0 & 0 \\
x1, x2, x4, x5, x6 & $-46(35.5)$ & 100 & 0 & 0 & 0 & 0 & 0 & 0 & 0 \\
x1, x3, x4, x5, x6 & $-94(38.4)$ & 100 & 0 & 0 & 0 & 0 & 0 & 0 & 0 \\
x2, x3, x4, x5, x6 & $183(25.5)$ & 2 & 98 & 0 & 0 & 0 & 0 & 0 & 0 \\
\hline
\end{tabular}

Table 5.21: Summary of model AICc by variable subset and the corresponding number of times each candidate bandwidth value $\gamma$ was chosen over the 100 simulated data sets for scenario 3 in case b) environmental correlation structure 3 .

\begin{tabular}{|c|c|c|c|c|c|c|c|c|c|}
\hline \multirow[b]{3}{*}{ Subset } & \multirow[b]{2}{*}{$\mathrm{AICc}$} & \multicolumn{8}{|c|}{ \# Times Selected } \\
\hline & & \multicolumn{8}{|c|}{ Bandwidth Value $\gamma$} \\
\hline & Mean (SD) & 0.1 & 0.2 & 0.3 & 0.5 & 0.6 & 0.8 & 1.0 & 1.4 \\
\hline$x 1, x 2, x 3$ & $316(37.8)$ & 16 & 84 & 0 & 0 & 0 & 0 & 0 & 0 \\
\hline$x 1, x 2, x 4$ & $353(35.9)$ & 1 & 99 & 0 & 0 & 0 & 0 & 0 & 0 \\
\hline$x 1, x 2, x 5$ & $338(35.6)$ & 7 & 93 & 0 & 0 & 0 & 0 & 0 & 0 \\
\hline$x 1, x 2, x 6$ & $-229(31.3)$ & 100 & 0 & 0 & 0 & 0 & 0 & 0 & 0 \\
\hline$x 1, x 3, x 4$ & $327(36.6)$ & 5 & 95 & 0 & 0 & 0 & 0 & 0 & 0 \\
\hline$x 1, x 3, x 5$ & $324(37.4)$ & 8 & 92 & 0 & 0 & 0 & 0 & 0 & 0 \\
\hline$x 1, x 3, x 6$ & $-259(32.4)$ & 100 & 0 & 0 & 0 & 0 & 0 & 0 & 0 \\
\hline$x 1, x 4, x 5$ & $365(35.4)$ & 1 & 99 & 0 & 0 & 0 & 0 & 0 & 0 \\
\hline$x 1, x 4, x 6$ & $-226(31.7)$ & 100 & 0 & 0 & 0 & 0 & 0 & 0 & 0 \\
\hline$x 1, x 5, x 6$ & $-222(32.4)$ & 100 & 0 & 0 & 0 & 0 & 0 & 0 & 0 \\
\hline$x 2, x 3, x 4$ & $457(35.1)$ & 0 & 100 & 0 & 0 & 0 & 0 & 0 & 0 \\
\hline$x 2, x 3, x 5$ & $434(35.8)$ & 0 & 100 & 0 & 0 & 0 & 0 & 0 & 0 \\
\hline$x 2, x 3, x 6$ & $-105(32.8)$ & 100 & 0 & 0 & 0 & 0 & 0 & 0 & 0 \\
\hline $\mathrm{x} 2, \mathrm{x} 4, \mathrm{x} 5$ & $476(35.5)$ & 0 & 100 & 0 & 0 & 0 & 0 & 0 & 0 \\
\hline $\mathrm{x} 2, \mathrm{x} 4, \mathrm{x} 6$ & $-73(31.1)$ & 100 & 0 & 0 & 0 & 0 & 0 & 0 & 0 \\
\hline $\mathrm{x} 2, \mathrm{x} 5, \mathrm{x} 6$ & $-75(33.1)$ & 100 & 0 & 0 & 0 & 0 & 0 & 0 & 0 \\
\hline$x 3, x 4, x 5$ & 469 (36.9) & 0 & 100 & 0 & 0 & 0 & 0 & 0 & 0 \\
\hline$x 3, x 4, x 6$ & $-99(32.3)$ & 100 & 0 & 0 & 0 & 0 & 0 & 0 & 0 \\
\hline$x 3, x 5, x 6$ & $-91(34.2)$ & 100 & 0 & 0 & 0 & 0 & 0 & 0 & 0 \\
\hline$x 4, x 5, x 6$ & $-58(32.2)$ & 100 & 0 & 0 & 0 & 0 & 0 & 0 & 0 \\
\hline $\mathrm{x} 1, \mathrm{x} 2, \mathrm{x} 3, \mathrm{x} 4$ & $327(36.1)$ & 0 & 100 & 0 & 0 & 0 & 0 & 0 & 0 \\
\hline $\mathrm{x} 1, \mathrm{x} 2, \mathrm{x} 3, \mathrm{x} 5$ & $317(35.5)$ & 1 & 99 & 0 & 0 & 0 & 0 & 0 & 0 \\
\hline $\mathrm{x} 1, \mathrm{x} 2, \mathrm{x} 3, \mathrm{x} 6$ & $-194(32.3)$ & 100 & 0 & 0 & 0 & 0 & 0 & 0 & 0 \\
\hline
\end{tabular}




\begin{tabular}{lrrrrrrrrr}
$\mathrm{x} 1, \mathrm{x} 2, \mathrm{x} 4, \mathrm{x} 5$ & $348(34.5)$ & 1 & 99 & 0 & 0 & 0 & 0 & 0 & 0 \\
$\mathrm{x} 1, \mathrm{x} 2, \mathrm{x} 4, \mathrm{x} 6$ & $-167(31.4)$ & 100 & 0 & 0 & 0 & 0 & 0 & 0 & 0 \\
$\mathrm{x} 1, \mathrm{x} 2, \mathrm{x} 5, \mathrm{x} 6$ & $-167(31.9)$ & 100 & 0 & 0 & 0 & 0 & 0 & 0 & 0 \\
$\mathrm{x} 1, \mathrm{x} 3, \mathrm{x} 4, \mathrm{x} 5$ & $328(35.6)$ & 1 & 99 & 0 & 0 & 0 & 0 & 0 & 0 \\
$\mathrm{x} 1, \mathrm{x} 3, \mathrm{x} 4, \mathrm{x} 6$ & $-194(32.8)$ & 100 & 0 & 0 & 0 & 0 & 0 & 0 & 0 \\
$\mathrm{x} 1, \mathrm{x} 3, \mathrm{x} 5, \mathrm{x} 6$ & $-193(33.0)$ & 100 & 0 & 0 & 0 & 0 & 0 & 0 & 0 \\
$\mathrm{x} 1, \mathrm{x} 4, \mathrm{x} 5, \mathrm{x} 6$ & $-163(32.0)$ & 100 & 0 & 0 & 0 & 0 & 0 & 0 & 0 \\
$\mathrm{x} 2, \mathrm{x} 3, \mathrm{x} 4, \mathrm{x} 5$ & $439(36.0)$ & 0 & 100 & 0 & 0 & 0 & 0 & 0 & 0 \\
$\mathrm{x} 2, \mathrm{x} 3, \mathrm{x} 4, \mathrm{x} 6$ & $-51(31.9)$ & 100 & 0 & 0 & 0 & 0 & 0 & 0 & 0 \\
$\mathrm{x} 2, \mathrm{x} 3, \mathrm{x} 5, \mathrm{x} 6$ & $-53(33.8)$ & 100 & 0 & 0 & 0 & 0 & 0 & 0 & 0 \\
$\mathrm{x} 2, \mathrm{x} 4, \mathrm{x} 5, \mathrm{x} 6$ & $-24(32.0)$ & 100 & 0 & 0 & 0 & 0 & 0 & 0 & 0 \\
$\mathrm{x} 3, \mathrm{x} 4, \mathrm{x} 5, \mathrm{x} 6$ & $-46(32.8)$ & 100 & 0 & 0 & 0 & 0 & 0 & 0 & 0 \\
& & & & & & & & & \\
$\mathrm{x} 1, \mathrm{x} 2, \mathrm{x} 3, \mathrm{x} 4, \mathrm{x} 5$ & $331(35.3)$ & 0 & 100 & 0 & 0 & 0 & 0 & 0 & 0 \\
$\mathrm{x} 1, \mathrm{x} 2, \mathrm{x} 3, \mathrm{x} 4, \mathrm{x} 6$ & $-127(32.8)$ & 100 & 0 & 0 & 0 & 0 & 0 & 0 & 0 \\
$\mathrm{x} 1, \mathrm{x} 2, \mathrm{x} 3, \mathrm{x} 5, \mathrm{x} 6$ & $-128(32.9)$ & 100 & 0 & 0 & 0 & 0 & 0 & 0 & 0 \\
$\mathrm{x} 1, \mathrm{x} 2, \mathrm{x} 4, \mathrm{x} 5, \mathrm{x} 6$ & $-103(31.9)$ & 100 & 0 & 0 & 0 & 0 & 0 & 0 & 0 \\
$\mathrm{x} 1, \mathrm{x} 3, \mathrm{x} 4, \mathrm{x} 5, \mathrm{x} 6$ & $-127(33.1)$ & 100 & 0 & 0 & 0 & 0 & 0 & 0 & 0 \\
$\mathrm{x} 2, \mathrm{x} 3, \mathrm{x} 4, \mathrm{x} 5, \mathrm{x} 6$ & $4(32.6)$ & 98 & 2 & 0 & 0 & 0 & 0 & 0 & 0 \\
\hline
\end{tabular}

As shown in the left side of Table 5.22, average weighted AICc is smallest for subsets of size 5 in the first correlation case. Thus, the ensemble estimates for this case are selected as those calculated using subsets of size 5 . In the second correlation case, we see that while weighted AICc is minimized for subsets of size 4 , the values do not differ largely across subset size (see right side of Table 5.22). We select the ensemble estimates calculated using subsets of size 4 for presentation in the second correlation case, but note that future research into the choice of variable subset size is needed. Finally, we see that regardless of correlation pattern, the AICc from the best subset is far lower than the weighted average AICc for each of the three subset sizes, again suggesting a superior goodness-of-fit for the best subset model. 
Table 5.22: Summary of model AICc in simulation scenario 3 for the best subset method and for ensemble methods using variable subset sizes of 3, 4, and 5 for the cases of a) environmental correlation matrix 2 and b) environmental correlation matrix 3.

\begin{tabular}{lrrrr} 
& & Case a & Case b \\
\cline { 2 - 3 } Ensemble Method & Mean (SD) & Mean (SD) \\
\cline { 2 - 3 } & \# of subsets & Weighted AICc & Weighted AICc \\
\hline Size 3 & 20 & $66(25.5)$ & & $-95(25.7)$ \\
Size 4 & 15 & $-18(30.2)$ & & $-113(28.5)$ \\
Size 5 & 6 & $-51(34.8)$ & & $-100(31.2)$ \\
& & & \\
Best Subset & Bandwidth & AICc & AICc \\
\hline x1, x3, x6 & 0.1 & $-230(36.4)$ & $-259(32.4)$ \\
\hline
\end{tabular}

The best subset estimates are mapped over the study region in Figure 5.15. In both correlation cases, the parameter estimates are able to replicate the general spatial pattern in the mixture effect and in the parameter weights. However, as discussed in scenario 2, the estimates are unable to detect the linear boundaries between rows due to the use of a smooth kernel function. As demonstrated in the parameter maps, this over-smoothing leads to visibly diminished distinction between rows.
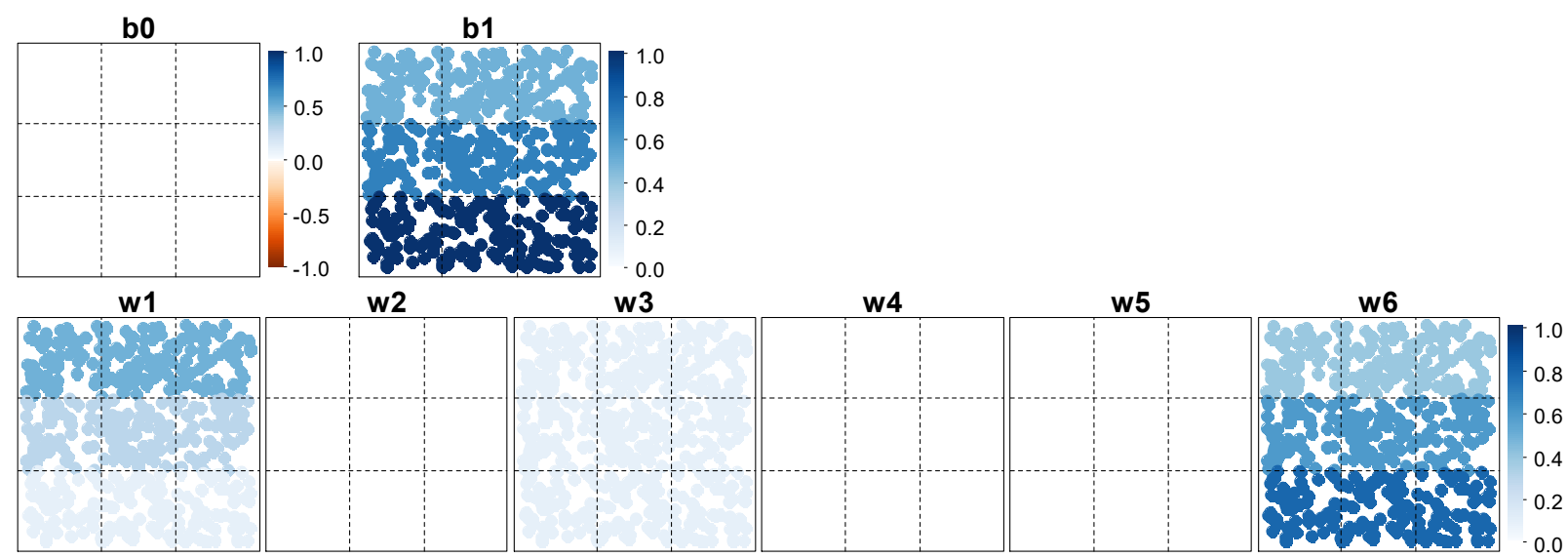

Panel 1. Simulated LWQS parameter values in scenario 3. 


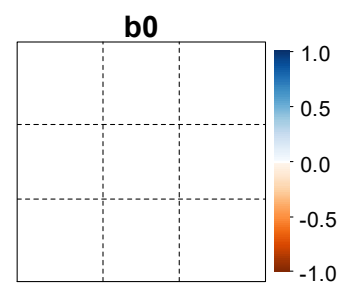

w1

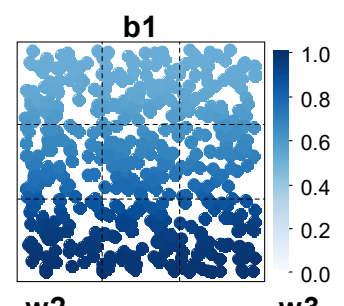
w3

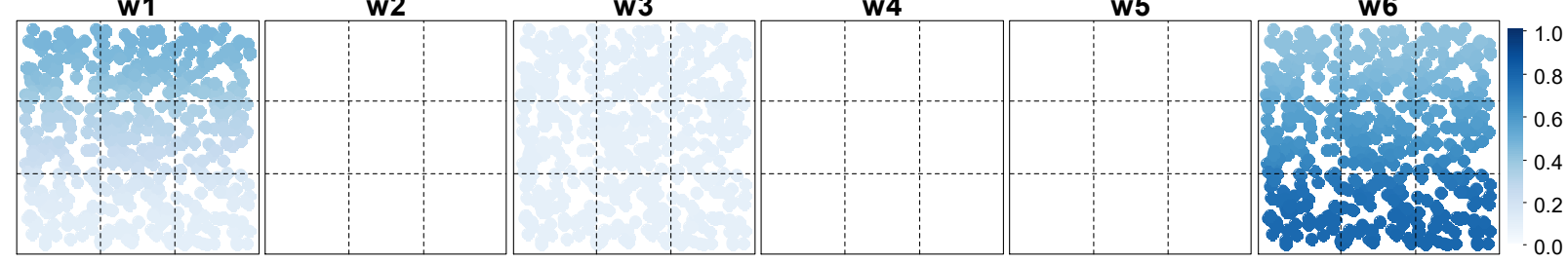

Panel 2. Best subset parameter estimates for case a) environmental correlation matrix 2.

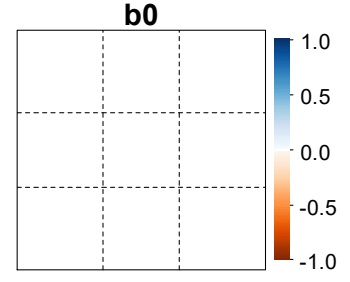

w1

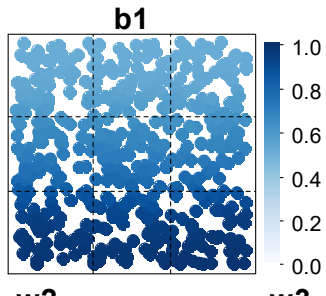

w2

w3
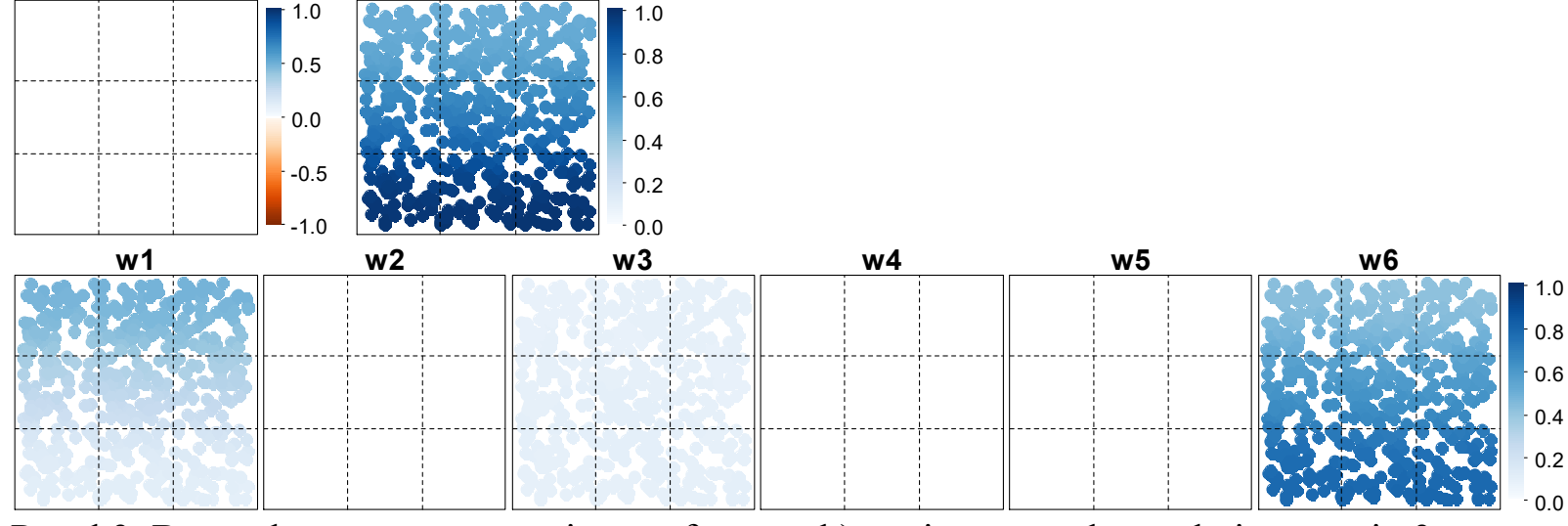

Panel 3. Best subset parameter estimates for case b) environmental correlation matrix 3 .

Figure 5.15: Maps of the LWQS best subset parameter estimates in simulation scenario 3 for cases a) environmental correlation matrix 2 (panel 2), and b) environmental correlation matrix 3 (panel 3). The simulated LWQS parameters for scenario 3 are included for the purpose of comparison (panel 1).

The parameter estimates are summarized by row of the study region for each correlation case in

Table 5.23 with error in summarized similarly in Table 5.24. On average, the best subset model correctly estimates the intercept, $\mathrm{b} 0$, and weight parameter for $\mathrm{x} 3, \mathrm{w} 3$, with little to no bias in both correlation cases. The average estimates of the spatially dependent parameters (b1, w1, and w6) provide further evidence of over-smoothing, with underestimates in regions of high activity and over estimates in regions of low activity. This is clearly visible in Figure 5.16, where the bias in the best subset parameter estimates is mapped over the study region. The reversal in the 
direction of bias near row divisions signals the estimates' difficulty in detecting linear boundaries. The magnitude of bias, however, is relatively consistent across correlation cases.

Table 5.23: Summary of the LWQS parameter estimates for simulation scenario 3 using the best subset $^{1}$ and ensemble ${ }^{2}$ methods in the cases of a) environmental correlation matrix 2 and $b$ ) environmental correlation matrix 3 .

\begin{tabular}{|c|c|c|c|c|c|c|c|}
\hline \multirow{2}{*}{\multicolumn{2}{|c|}{ Case a }} & \multicolumn{3}{|c|}{ Best Subset Estimates } & \multicolumn{3}{|c|}{ Ensemble Estimates } \\
\hline & & Truth & Mean & Range & Truth & Mean & Range \\
\hline \multirow[t]{3}{*}{ b0 } & Row 1 & 0 & 0.00 & $(0.00,0.00)$ & 0 & 0.00 & $(-0.01,0.00)$ \\
\hline & Row 2 & 0 & 0.00 & $(-0.01,0.00)$ & 0 & -0.01 & $(-0.02,0.00)$ \\
\hline & Row 3 & 0 & 0.00 & $(-0.01,0.01)$ & 0 & -0.01 & $(-0.02,0.00)$ \\
\hline \multirow[t]{3}{*}{ b1 } & Row 1 & 0.5 & 0.54 & $(0.51,0.62)$ & 0.5 & 0.55 & $(0.52,0.63)$ \\
\hline & Row 2 & 0.7 & 0.71 & $(0.62,0.85)$ & 0.7 & 0.71 & $(0.63,0.85)$ \\
\hline & Row 3 & 1 & 0.95 & $(0.84,0.99)$ & 1 & 0.94 & $(0.85,0.98)$ \\
\hline \multirow[t]{3}{*}{ w1 } & Row 1 & 0.5 & 0.44 & $(0.35,0.48)$ & 0.5 & 0.40 & $(0.32,0.44)$ \\
\hline & Row 2 & 0.3 & 0.29 & $(0.18,0.36)$ & 0.3 & 0.27 & $(0.16,0.33)$ \\
\hline & Row 3 & 0.1 & 0.13 & $(0.11,0.19)$ & 0.1 & 0.12 & $(0.10,0.18)$ \\
\hline \multirow[t]{3}{*}{ w2 } & Row 1 & 0 & 0 & $(0,0)$ & 0 & 0.03 & $(0.02,0.03)$ \\
\hline & Row 2 & 0 & 0 & $(0,0)$ & 0 & 0.02 & $(0.02,0.03)$ \\
\hline & Row 3 & 0 & 0 & $(0,0)$ & 0 & 0.02 & $(0.02,0.03)$ \\
\hline \multirow[t]{3}{*}{ w3 } & Row 1 & 0.1 & 0.10 & $(0.10,0.11)$ & 0.1 & 0.09 & $(0.09,0.10)$ \\
\hline & Row 2 & 0.1 & 0.10 & $(0.09,0.11)$ & 0.1 & 0.09 & $(0.08,0.09)$ \\
\hline & Row 3 & 0.1 & 0.10 & $(0.09,0.10)$ & 0.1 & 0.08 & $(0.07,0.08)$ \\
\hline \multirow[t]{3}{*}{ w4 } & Row 1 & 0 & 0 & $(0,0)$ & 0 & 0.01 & $(0.01,0.02)$ \\
\hline & Row 2 & 0 & 0 & $(0,0)$ & 0 & 0.01 & $(0.01,0.02)$ \\
\hline & Row 3 & 0 & 0 & $(0,0)$ & 0 & 0.01 & $(0.01,0.02)$ \\
\hline \multirow[t]{3}{*}{ w5 } & Row 1 & 0 & 0 & $(0,0)$ & 0 & 0.02 & $(0.02,0.03)$ \\
\hline & Row 2 & 0 & 0 & $(0,0)$ & 0 & 0.03 & $(0.02,0.04)$ \\
\hline & Row 3 & 0 & 0 & $(0,0)$ & 0 & 0.02 & $(0.02,0.04)$ \\
\hline \multirow[t]{3}{*}{ w6 } & Row 1 & 0.4 & 0.45 & $(0.42,0.54)$ & 0.4 & 0.44 & $(0.40,0.51)$ \\
\hline & Row 2 & 0.6 & 0.61 & $(0.54,0.72)$ & 0.6 & 0.59 & $(0.51,0.68)$ \\
\hline & Row 3 & 0.8 & 0.77 & $(0.71,0.79)$ & 0.8 & 0.74 & $(0.67,0.77)$ \\
\hline \multicolumn{8}{|c|}{ Case b } \\
\hline \multirow[t]{3}{*}{ b0 } & Row 1 & 0 & 0.00 & $(0.00,0.01)$ & 0 & 0.01 & $(0.00,0.01)$ \\
\hline & Row 2 & 0 & 0.00 & $(0.00,0.00)$ & 0 & 0.00 & $(0.00,0.01)$ \\
\hline & Row 3 & 0 & 0.00 & $(-0.01,0.00)$ & 0 & 0.00 & $(0.00,0.01)$ \\
\hline \multirow[t]{3}{*}{ b1 } & Row 1 & 0.5 & 0.54 & $(0.51,0.62)$ & 0.5 & 0.54 & $(0.51,0.62)$ \\
\hline & Row 2 & 0.7 & 0.71 & $(0.62,0.85)$ & 0.7 & 0.71 & $(0.62,0.85)$ \\
\hline & Row 3 & 1 & 0.94 & $(0.84,0.99)$ & 1 & 0.94 & $(0.84,0.98)$ \\
\hline \multirow[t]{2}{*}{ w1 } & Row 1 & 0.5 & 0.45 & $(0.36,0.49)$ & 0.5 & 0.34 & $(0.28,0.36)$ \\
\hline & Row 2 & 0.3 & 0.29 & $(0.19,0.37)$ & 0.3 & 0.23 & $(0.16,0.28)$ \\
\hline
\end{tabular}




\begin{tabular}{rrrrrrrr}
\multirow{2}{*}{ w2 } & Row 3 & 0.1 & 0.13 & $(0.11,0.19)$ & 0.1 & 0.12 & $(0.10,0.16)$ \\
& Row 1 & 0 & 0 & $(0,0)$ & 0 & 0.05 & $(0.04,0.06)$ \\
& Row 2 & 0 & 0 & $(0,0)$ & 0 & 0.04 & $(0.03,0.05)$ \\
W3 & Row 3 & 0 & 0 & $(0,0)$ & 0 & 0.03 & $(0.03,0.04)$ \\
& Row 1 & 0.1 & 0.09 & $(0.08,0.10)$ & 0.1 & 0.07 & $(0.07,0.08)$ \\
& Row 2 & 0.1 & 0.10 & $(0.09,0.11)$ & 0.1 & 0.07 & $(0.07,0.08)$ \\
w4 & Row 3 & 0.1 & 0.10 & $(0.09,0.11)$ & 0.1 & 0.07 & $(0.07,0.08)$ \\
Row 1 & 0 & 0 & $(0,0)$ & 0 & 0.03 & $(0.03,0.04)$ \\
& Row 2 & 0 & 0 & $(0,0)$ & 0 & 0.03 & $(0.02,0.03)$ \\
w5 & Row 3 & 0 & 0 & $(0,0)$ & 0 & 0.02 & $(0.02,0.03)$ \\
& Row 1 & 0 & 0 & $(0,0)$ & 0 & 0.03 & $(0.02,0.03)$ \\
& Row 2 & 0 & 0 & $(0,0)$ & 0 & 0.02 & $(0.02,0.03)$ \\
Row 3 & 0 & 0 & $(0,0)$ & 0 & 0.02 & $(0.02,0.03)$ \\
w6 & Row 1 & 0.4 & 0.46 & $(0.42,0.55)$ & 0.4 & 0.48 & $(0.44,0.55)$ \\
& Row 2 & 0.6 & 0.61 & $(0.54,0.70)$ & 0.6 & 0.61 & $(0.55,0.68)$ \\
& Row 3 & 0.8 & 0.77 & $(0.71,0.79)$ & 0.8 & 0.74 & $(0.68,0.76)$ \\
\hline
\end{tabular}

${ }^{1}$ Best subset estimates pertain to $\mathrm{S}_{\mathrm{b}}=\{\mathrm{x} 1, \mathrm{x} 3, \mathrm{x} 6\}$ with estimated bandwidth value $\gamma=0.1$.

${ }^{2}$ Ensemble estimates are calculated using subsets of size 5 in case a and size 4 in case $b$

Table 5.24: Summary of the bias and RMSE in the estimated LWQS parameters for simulation scenario 3 using the best subset ${ }^{1}$ and ensemble ${ }^{2}$ methods for the cases of a) environmental correlation matrix 2 and b) environmental correlation matrix 3.

\begin{tabular}{rrrrrrrrrr} 
& & \multicolumn{3}{c}{ Best Subset Estimates } & & \multicolumn{3}{c}{ Ensemble Estimates } \\
\cline { 2 - 4 } \cline { 8 - 9 } Case a & Avg. Bias & Bias Range & RMSE & & Avg. Bias & Bias Range & RMSE \\
\hline b0 Row 1 & 0.00 & $(0.00,0.00)$ & 0.00 & & 0.00 & $(-0.01,0.00)$ & 0.00 \\
& Row 2 & 0.00 & $(-0.01,0.00)$ & 0.00 & & -0.01 & $(-0.02,0.00)$ & 0.01 \\
& Row 3 & 0.00 & $(-0.01,0.01)$ & 0.00 & & -0.01 & $(-0.02,0.00)$ & 0.01 \\
b1 & Row 1 & 0.04 & $(0.01,0.12)$ & 0.05 & & 0.05 & $(0.02,0.13)$ & 0.06 \\
& Row 2 & 0.01 & $(-0.08,0.15)$ & 0.06 & & 0.01 & $(-0.07,0.15)$ & 0.06 \\
& Row 3 & -0.05 & $(-0.16,-0.01)$ & 0.07 & & -0.06 & $(-0.15,-0.02)$ & 0.07 \\
w1 & Row 1 & -0.06 & $(-0.15,-0.02)$ & 0.07 & & -0.10 & $(-0.18,-0.06)$ & 0.10 \\
& Row 2 & -0.01 & $(-0.12,0.06)$ & 0.04 & & -0.03 & $(-0.14,0.03)$ & 0.05 \\
& Row 3 & 0.03 & $(0.01,0.09)$ & 0.04 & & 0.02 & $(0.00,0.08)$ & 0.03 \\
w2 & Row 1 & 0 & $(0,0)$ & 0 & & 0.03 & $(0.02,0.03)$ & 0.03 \\
& Row 2 & 0 & $(0,0)$ & 0 & & 0.02 & $(0.02,0.03)$ & 0.02 \\
& Row 3 & 0 & $(0,0)$ & 0 & & 0.02 & $(0.02,0.03)$ & 0.02 \\
w3 & Row 1 & 0.00 & $(0.00,0.01)$ & 0.01 & & -0.01 & $(-0.01,0.00)$ & 0.01 \\
& Row 2 & 0.00 & $(-0.01,0.01)$ & 0.00 & & -0.01 & $(-0.02,-0.01)$ & 0.02 \\
& Row 3 & 0.00 & $(-0.01,0.00)$ & 0.00 & & -0.02 & $(-0.03,-0.02)$ & 0.02 \\
w4 & Row 1 & 0 & $(0,0)$ & 0 & & 0.01 & $(0.01,0.02)$ & 0.01 \\
& Row 2 & 0 & $(0,0)$ & 0 & 0.01 & $(0.01,0.02)$ & 0.01 \\
& Row 3 & 0 & $(0,0)$ & 0 & 0.01 & $(0.01,0.02)$ & 0.01
\end{tabular}




\begin{tabular}{|c|c|c|c|c|c|c|c|}
\hline \multirow[t]{3}{*}{ w5 } & Row 1 & 0 & $(0,0)$ & 0 & 0.02 & $(0.02,0.03)$ & 0.02 \\
\hline & Row 2 & 0 & $(0,0)$ & 0 & 0.03 & $(0.02,0.04)$ & 0.03 \\
\hline & Row 3 & 0 & $(0,0)$ & 0 & 0.02 & $(0.02,0.04)$ & 0.02 \\
\hline \multirow[t]{3}{*}{ w6 } & Row 1 & 0.05 & $(0.02,0.14)$ & 0.06 & 0.04 & $(0.00,0.11)$ & 0.05 \\
\hline & Row 2 & 0.01 & $(-0.06,0.12)$ & 0.05 & -0.01 & $(-0.09,0.08)$ & 0.04 \\
\hline & Row 3 & -0.03 & $(-0.09,-0.01)$ & 0.04 & -0.06 & $(-0.13,-0.03)$ & 0.06 \\
\hline \multicolumn{8}{|c|}{ Case b } \\
\hline \multirow[t]{3}{*}{ b0 } & Row 1 & 0.00 & $(0.00,0.01)$ & 0.00 & 0.01 & $(0.00,0.01)$ & 0.01 \\
\hline & Row 2 & 0.00 & $(0.00,0.00)$ & 0.00 & 0.00 & $(0.00,0.01)$ & 0.00 \\
\hline & Row 3 & 0.00 & $(-0.01,0.00)$ & 0.00 & 0.00 & $(0.00,0.01)$ & 0.00 \\
\hline \multirow[t]{3}{*}{$\mathrm{b} 1$} & Row 1 & 0.04 & $(0.01,0.12)$ & 0.05 & 0.04 & $(0.01,0.12)$ & 0.05 \\
\hline & Row 2 & 0.01 & $(-0.08,0.15)$ & 0.06 & 0.01 & $(-0.08,0.15)$ & 0.06 \\
\hline & Row 3 & -0.06 & $(-0.16,-0.01)$ & 0.07 & -0.06 & $(-0.16,-0.02)$ & 0.07 \\
\hline \multirow[t]{3}{*}{ w1 } & Row 1 & -0.05 & $(-0.14,-0.01)$ & 0.06 & -0.16 & $(-0.22,-0.14)$ & 0.16 \\
\hline & Row 2 & -0.01 & $(-0.11,0.07)$ & 0.05 & -0.07 & $(-0.14,-0.02)$ & 0.08 \\
\hline & Row 3 & 0.03 & $(0.01,0.09)$ & 0.04 & 0.02 & $(0.00,0.06)$ & 0.02 \\
\hline \multirow[t]{3}{*}{ w2 } & Row 1 & 0 & $(0,0)$ & 0 & 0.05 & $(0.04,0.06)$ & 0.05 \\
\hline & Row 2 & 0 & $(0,0)$ & 0 & 0.04 & $(0.03,0.05)$ & 0.04 \\
\hline & Row 3 & 0 & $(0,0)$ & 0 & 0.03 & $(0.03,0.04)$ & 0.03 \\
\hline \multirow[t]{3}{*}{ w3 } & Row 1 & -0.01 & $(-0.02,0.00)$ & 0.01 & -0.03 & $(-0.03,-0.02)$ & 0.03 \\
\hline & Row 2 & 0.00 & $(-0.01,0.01)$ & 0.01 & -0.03 & $(-0.03,-0.02)$ & 0.03 \\
\hline & Row 3 & 0.00 & $(-0.01,0.01)$ & 0.00 & -0.03 & $(-0.03,-0.02)$ & 0.03 \\
\hline \multirow[t]{3}{*}{ w4 } & Row 1 & 0 & $(0,0)$ & 0 & 0.03 & $(0.03,0.04)$ & 0.03 \\
\hline & Row 2 & 0 & $(0,0)$ & 0 & 0.03 & $(0.02,0.03)$ & 0.03 \\
\hline & Row 3 & 0 & $(0,0)$ & 0 & 0.02 & $(0.02,0.03)$ & 0.02 \\
\hline \multirow[t]{3}{*}{ w5 } & Row 1 & 0 & $(0,0)$ & 0 & 0.03 & $(0.02,0.03)$ & 0.03 \\
\hline & Row 2 & 0 & $(0,0)$ & 0 & 0.02 & $(0.02,0.03)$ & 0.02 \\
\hline & Row 3 & 0 & $(0,0)$ & 0 & 0.02 & $(0.02,0.03)$ & 0.02 \\
\hline \multirow[t]{3}{*}{ w6 } & Row 1 & 0.06 & $(0.02,0.15)$ & 0.07 & 0.08 & $(0.04,0.15)$ & 0.08 \\
\hline & Row 2 & 0.01 & $(-0.06,0.10)$ & 0.04 & 0.01 & $(-0.05,0.08)$ & 0.04 \\
\hline & Row 3 & -0.03 & $(-0.09,-0.01)$ & 0.04 & -0.06 & $(-0.12,-0.04)$ & 0.07 \\
\hline
\end{tabular}

${ }^{1}$ Best subset estimates pertain to $\mathrm{S}_{\mathrm{b}}=\{\mathrm{x} 1, \mathrm{x} 3, \mathrm{x} 6\}$ with estimated bandwidth value $\gamma=0.1$.

${ }^{2}$ Ensemble estimates are calculated using subsets of size 5 in case a and size 4 in case $b$. 

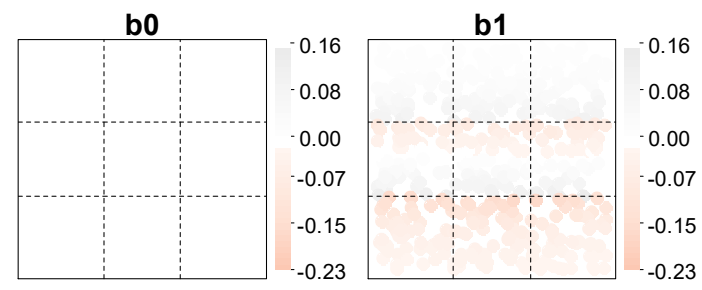

w1

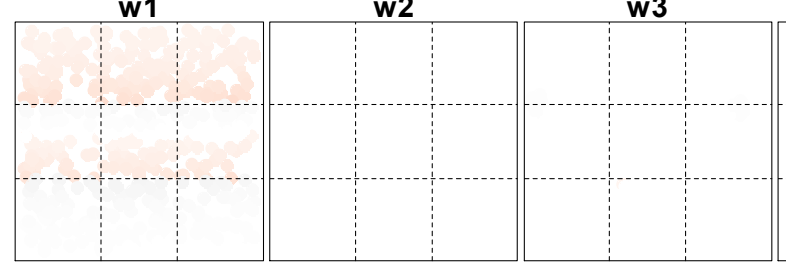

w3

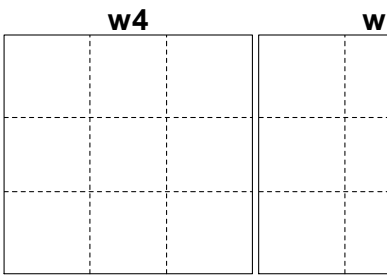

w5

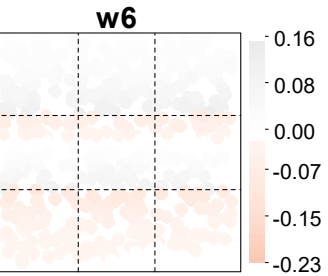

Panel 1. Bias in best subset parameter estimates for a) environmental correlation matrix 2.
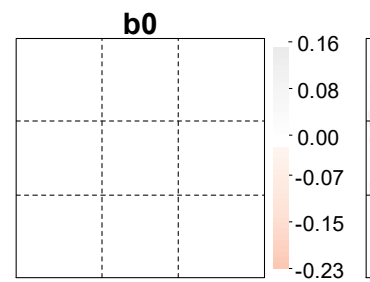

b1
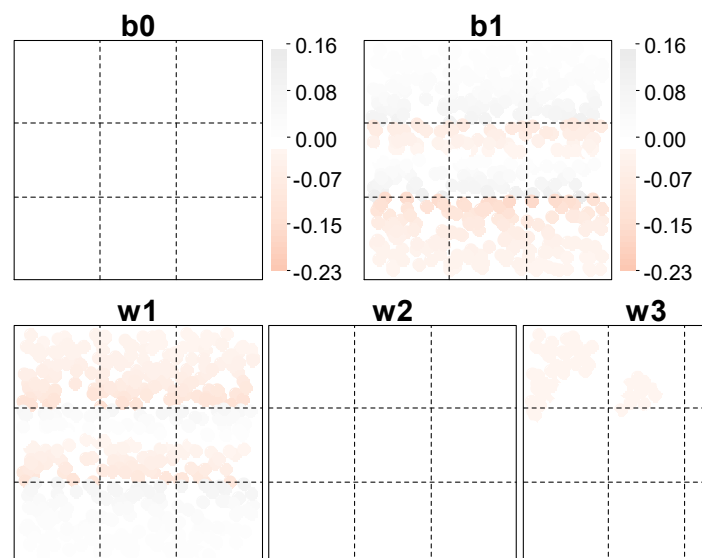

w3

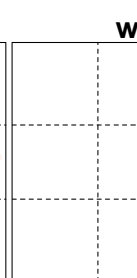

w4

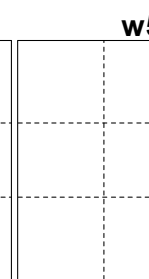

w5

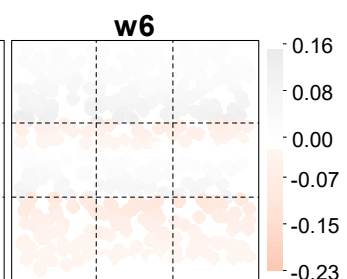

Panel 2. Bias in best subset parameter estimates for $b$ ) environmental correlation matrix 3

Figure 5.16: Bias in the best subset parameter estimates in scenario 3 for the cases of a) environmental correlation matrix 2 (panel 1) and b) environmental correlation matrix 3 (panel 2).

The ensemble estimates for both correlation cases are mapped over the study area in Figure

5.17, with bias shown in Figure 5.18. We note that in the first correlation case, the ensemble method produces a linear cluster of negatively estimated intercepts at the boundary of row 1 and row 2 . Further, while the estimates adequately detect the directional trend in the spatially varying parameters, the effects of over smoothing are particularly pronounced in the second correlation case. Examination of Tables 5.23 and $\mathbf{5 . 2 4}$ reveals that the bias is greatest in row 1 of w1 in both correlation cases. More specifically, the ensemble solutions underestimate w1 in row 1 by 0.10 in the first correlation case, and 0.16 in the second correlation case. The exaggerated bias is 
potentially attributable to an overall signal reduction in row 1 of the study region, where the mixture effect is at its weakest $(\mathrm{b} 1=0.5$ in row 1$)$.

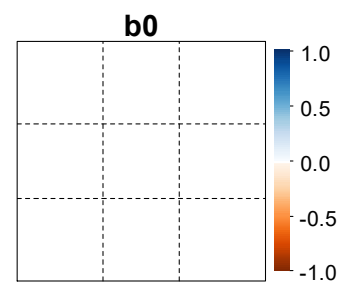

w1

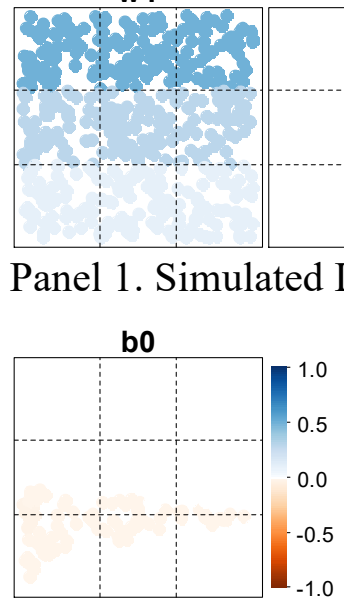

w1

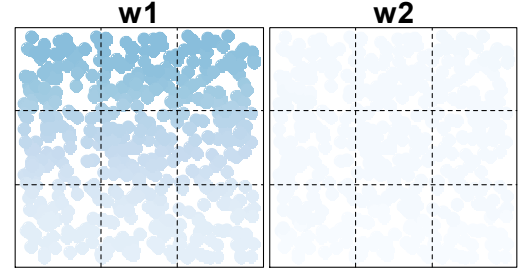

Panel 2. Ensemble estimates from subsets of size 5 for a) environmental correlation matrix 2.

b1

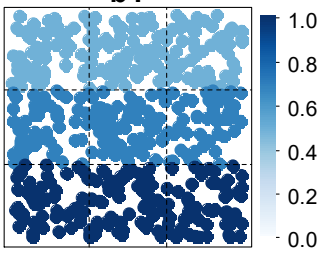

w2

w3

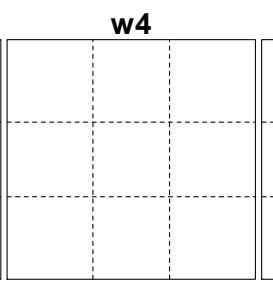

w5

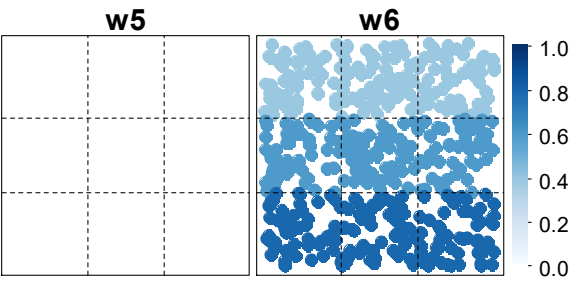

WQS parameter values in scenario 3.

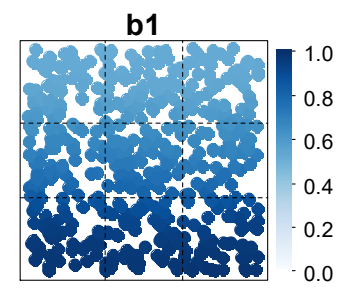

w3

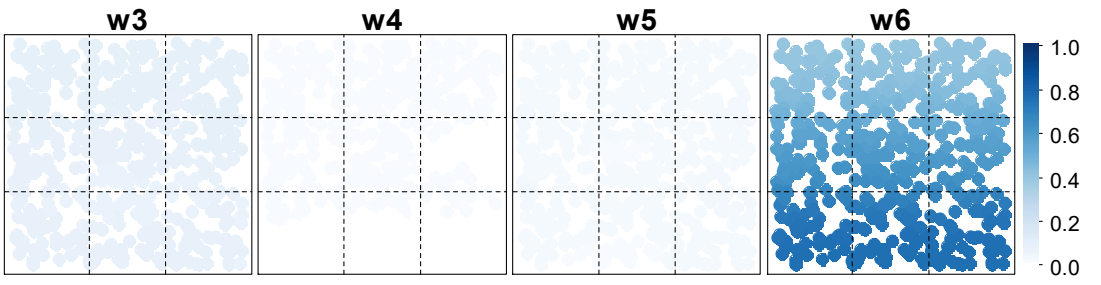

\section{.}



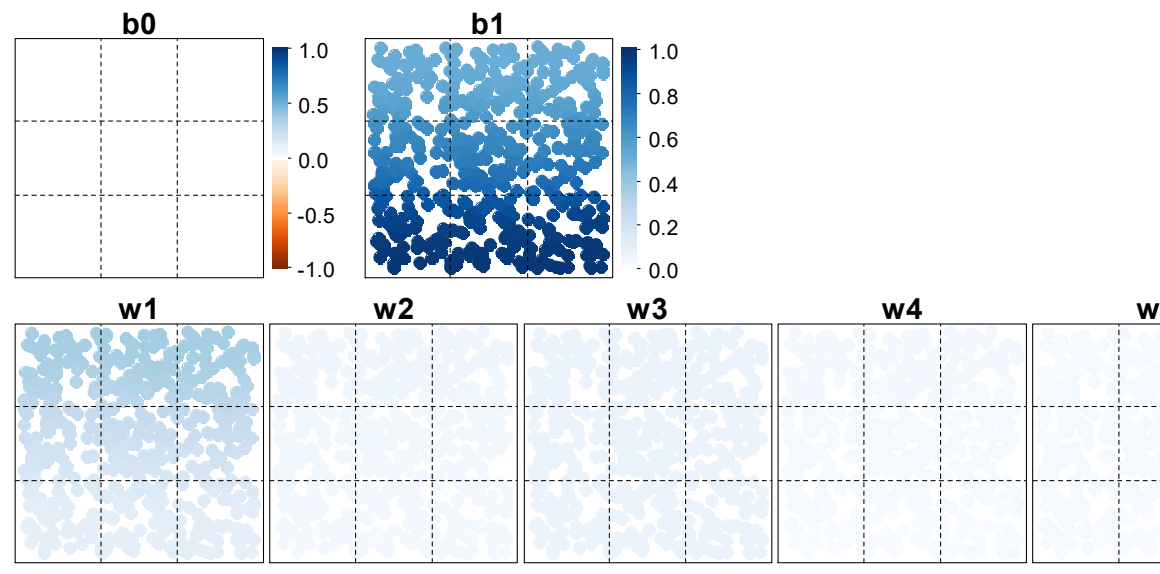

w5

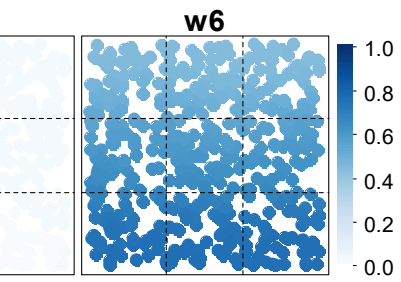

Panel 3. Ensemble estimates from subsets of size 4 for $b$ ) environmental correlation matrix 3.

Figure 5.17: Maps of the ensemble parameter estimates in simulation scenario 3 for cases a) environmental correlation matrix 2 (panel 2) and b) environmental correlation matrix 3 (panel 3). The simulated LWQS parameters for scenario 3 are included for the purpose of comparison (panel 1).
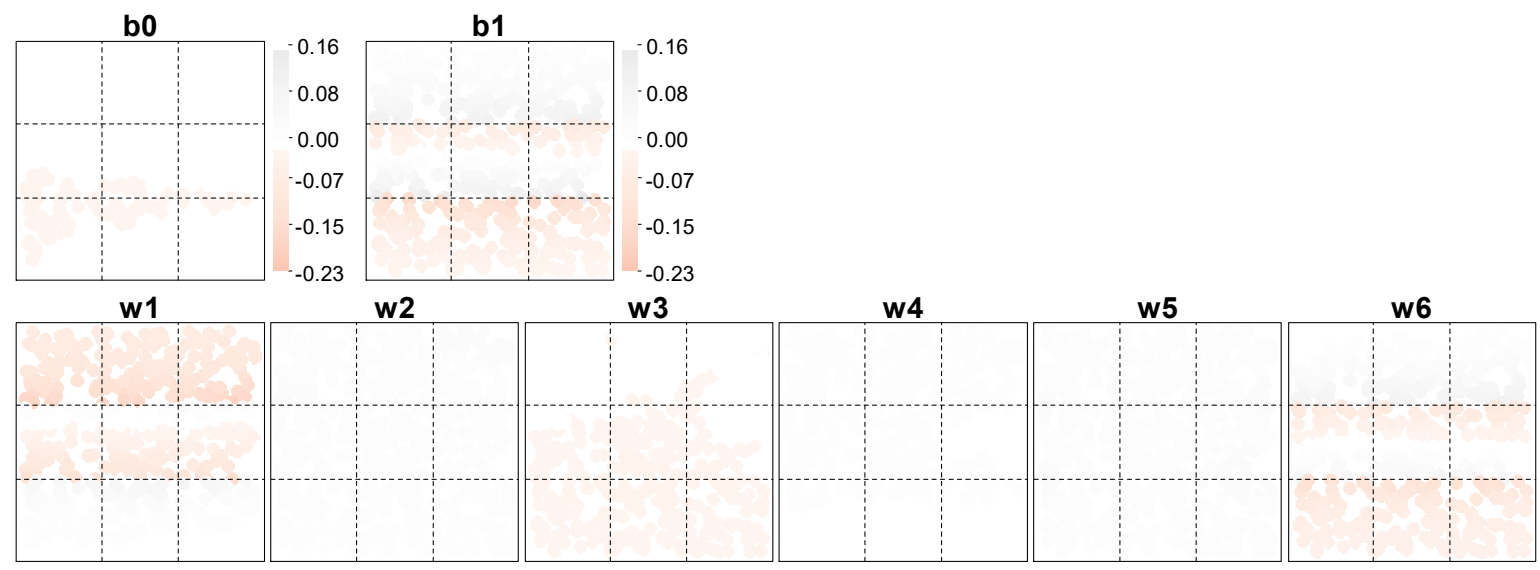

Panel 1. Bias in ensemble parameter estimates from subsets of size 5 for a) environmental correlation matrix 2 


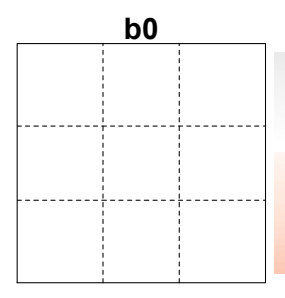

w1

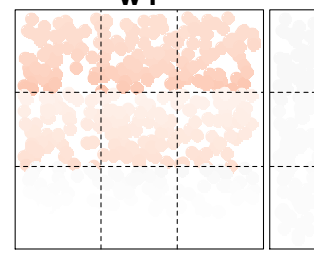

b1

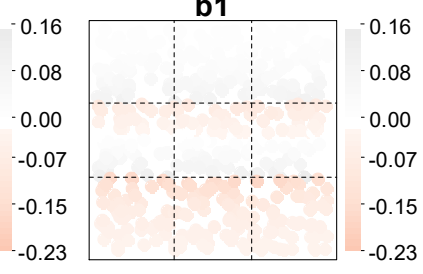

w2 w3

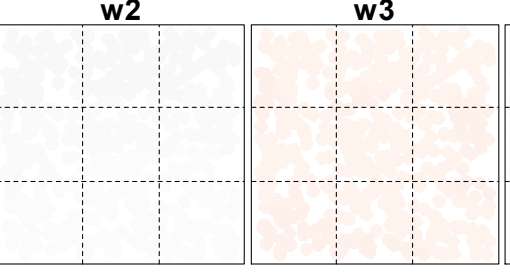

\begin{tabular}{|l|l|l|}
\multicolumn{1}{c|}{ w4 } \\
\hline & & \\
& & \\
\hdashline & & \\
\hdashline & & \\
\hline & & \\
\hline
\end{tabular}

w5

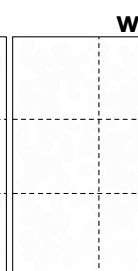

5

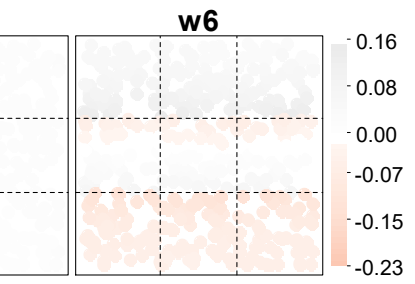

Panel 2. Bias in ensemble parameter estimates from subsets of size 4 for b) environmental correlation matrix 3

Figure 5.18: Bias in ensemble parameter estimates from subsets of size 5 in scenario 3 for the cases of a) environmental correlation matrix 2 (panel 1) and b) environmental correlation matrix 3 (panel 2).

As our last point of discussion, we draw attention to the ensemble method's overestimation of the weight parameters for $\mathrm{x} 2, \mathrm{x} 4$, and $\mathrm{x} 5$, particularly in the second correlation case. Using a cutoff of 0.05 to determine variable importance, the average ensemble estimates correctly distinguish between all important and unimportant exposures in the first correlation case. However, in the second more complicated correlation case, w2 is incorrectly classified as an important chemical in 146 of the 170 locations (i.e., 86\%) in row 1, and in two locations in row 2. We note however that the misclassification may be attributable to the use of subsets of size 4 in the calculation of the ensemble estimates, rather than the correlation structure.

In scenarios 1,2, and 3, LWQS has demonstrated the ability to replicate spatially dependent effects in the presence of highly correlated exposures. Furthermore, the best subset solutions perform accurate variable selection in terms of both sensitivity and specificity in all 3 scenarios. We now turn our attention to scenario 4 and 5, which assume smooth spatial dependency in exposures, the mixture effect (scenario $4 \& 5$ ), and the weight parameters (scenario 5). 


\section{Scenario 4}

In scenario 4, exposures $\mathrm{x} 2, \mathrm{x} 4$, and $\mathrm{x} 6$ vary systematically as a function of the distance from the center of the study region. Concentrations for these variables are highest in the center of the study area and decrease outwardly in a radial pattern. We assume no spatial variation in exposures $\mathrm{x} 1, \mathrm{x} 3$, and $\mathrm{x} 5$, and the weight parameters for all exposures are constant over the study region. The mixture effect is strongest in the center of the study area and decreases outwardly in a pattern similar to that of the spatially varying exposures.

Model AICc and choice of bandwidth parameter are summarized for all subsets in Table 5.25. The best subset was correctly chosen as $\mathrm{S}_{\mathrm{b}}=\{\mathrm{x} 1, \mathrm{x} 3, \mathrm{x} 6\}$, with the minimum average AICc across all subsets models. The estimated bandwidth parameters were largely limited to the two smallest candidate values $(\gamma=0.1$, and 0.2$)$, with the exception of the subset containing exposures x2, x4, and x6. As shown in Table 5.26, the weighted AICc is minimized for subsets of size 4 , and thus, the ensemble estimates calculated using subsets of size 4 are selected for presentation. We also note that the AICc for the best subset is again far lower than the weighted AICc for any subset size, suggesting superior goodness-of-fit for the best subset model.

Table 5.25: Summary of model AICc by variable subset and the corresponding number of times each candidate bandwidth value $\gamma$ was chosen over the 100 simulated data sets for scenario 4 .

\begin{tabular}{|c|c|c|c|c|c|c|c|c|c|}
\hline \multirow[b]{3}{*}{ Subset } & \multirow[b]{2}{*}{ AICc } & \multicolumn{8}{|c|}{ \# Times Selected } \\
\hline & & \multicolumn{8}{|c|}{ Bandwidth Value $\gamma$} \\
\hline & Mean (SD) & 0.1 & 0.2 & 0.3 & 0.5 & 0.6 & 0.8 & 1.0 & 1.4 \\
\hline $\mathrm{x} 1, \mathrm{x} 2, \mathrm{x} 3$ & $402(23.8)$ & 100 & 0 & 0 & 0 & 0 & 0 & 0 & 0 \\
\hline $\mathrm{x} 1, \mathrm{x} 2, \mathrm{x} 4$ & $397(21.7)$ & 100 & 0 & 0 & 0 & 0 & 0 & 0 & 0 \\
\hline$x 1, x 2, x 5$ & $412(23.8)$ & 100 & 0 & 0 & 0 & 0 & 0 & 0 & 0 \\
\hline$x 1, x 2, x 6$ & $-154(21.7)$ & 100 & 0 & 0 & 0 & 0 & 0 & 0 & 0 \\
\hline$x 1, x 3, x 4$ & $437(20.8)$ & 100 & 0 & 0 & 0 & 0 & 0 & 0 & 0 \\
\hline$x 1, x 3, x 5$ & $448(22.5)$ & 100 & 0 & 0 & 0 & 0 & 0 & 0 & 0 \\
\hline$x 1, x 3, x 6$ & $-172(21.8)$ & 100 & 0 & 0 & 0 & 0 & 0 & 0 & 0 \\
\hline $\mathrm{x} 1, \mathrm{x} 4, \mathrm{x} 5$ & $445(22.0)$ & 100 & 0 & 0 & 0 & 0 & 0 & 0 & 0 \\
\hline $\mathrm{x} 1, \mathrm{x} 4, \mathrm{x} 6$ & $-149(22.2)$ & 100 & 0 & 0 & 0 & 0 & 0 & 0 & 0 \\
\hline
\end{tabular}




\begin{tabular}{|c|c|c|c|c|c|c|c|c|}
\hline$x 1, x 5, x 6$ & $-152(22.3)$ & 100 & 0 & 0 & 0 & 0 & 0 & 0 \\
\hline$x 2, x 3, x 4$ & $441(26.0)$ & 100 & 0 & 0 & 0 & 0 & 0 & 0 \\
\hline$x 2, x 3, x 5$ & $441(26.9)$ & 100 & 0 & 0 & 0 & 0 & 0 & U \\
\hline$x 2, x 3, x 6$ & $-34(26.1)$ & 100 & 0 & 0 & 0 & 0 & 0 & \\
\hline$x 2, x 4, x 5$ & $473(27.1)$ & 100 & 0 & 0 & 0 & 0 & 0 & U \\
\hline$x 2, x 4, x 6$ & $304(25.0)$ & 0 & 54 & 37 & 7 & 2 & 0 & ) \\
\hline$x 2, x 5, x 6$ & $48(30.0)$ & 100 & 0 & 0 & 0 & 0 & 0 & U \\
\hline$x 3, x 4, x 5$ & $473(24.0)$ & 100 & 0 & 0 & 0 & 0 & 0 & J \\
\hline$x 3, x 4, x 6$ & $-29(27.5)$ & 100 & 0 & 0 & 0 & 0 & 0 & 0 \\
\hline$x 3, x 5, x 6$ & $-60(24.5)$ & 100 & 0 & 0 & 0 & 0 & 0 & J \\
\hline$x 4, x 5, x 6$ & $53(29.7)$ & 100 & 0 & 0 & 0 & 0 & 0 & 0 \\
\hline $\mathrm{x} 1, \mathrm{x} 2, \mathrm{x} 3, \mathrm{x} 4$ & $446(23.7)$ & 100 & 0 & 0 & 0 & 0 & 0 & \\
\hline $\mathrm{x} 1, \mathrm{x} 2, \mathrm{x} 3, \mathrm{x} 5$ & $466(25.7)$ & 100 & 0 & 0 & 0 & 0 & 0 & 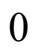 \\
\hline$x 1, x 2, x 3, x 6$ & $-118(22.0)$ & 100 & 0 & 0 & 0 & 0 & 0 & 0 \\
\hline$x 1, x 2, x 4, x 5$ & $455(23.8)$ & 100 & 0 & 0 & 0 & 0 & 0 & 0 \\
\hline$x 1, x 2, x 4, x 6$ & $-95(22.7)$ & 100 & 0 & 0 & 0 & 0 & 0 & $\mathrm{~J}$ \\
\hline$x 1, x 2, x 5, x 6$ & $-98(22.5)$ & 100 & 0 & 0 & 0 & 0 & 0 & 0 \\
\hline $\mathrm{x} 1, \mathrm{x} 3, \mathrm{x} 4, \mathrm{x} 5$ & $501(23.3)$ & 100 & 0 & 0 & 0 & 0 & 0 & J \\
\hline $\mathrm{x} 1, \mathrm{x} 3, \mathrm{x} 4, \mathrm{x} 6$ & $-113(22.8)$ & 100 & 0 & 0 & 0 & 0 & 0 & J \\
\hline$x 1, x 3, x 5, x 6$ & $-108(22.3)$ & 100 & 0 & 0 & 0 & 0 & 0 & J \\
\hline $\mathrm{x} 1, \mathrm{x} 4, \mathrm{x} 5, \mathrm{x} 6$ & $-94(23.1)$ & 100 & 0 & 0 & 0 & 0 & 0 & 0 \\
\hline$x 2, x 3, x 4, x 5$ & $484(27.4)$ & 100 & 0 & 0 & 0 & 0 & 0 & 0 \\
\hline$x 2, x 3, x 4, x 6$ & $25(27.2)$ & 98 & 2 & 0 & 0 & 0 & 0 & 0 \\
\hline$x 2, x 3, x 5, x 6$ & $-6(25.3)$ & 100 & 0 & 0 & 0 & 0 & 0 & 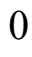 \\
\hline$x 2, x 4, x 5, x 6$ & $101(28.0)$ & 49 & 51 & 0 & 0 & 0 & 0 & 0 \\
\hline $\mathrm{x} 3, \mathrm{x} 4, \mathrm{x} 5, \mathrm{x} 6$ & $-1(25.9)$ & 100 & 0 & 0 & 0 & 0 & 0 & 0 \\
\hline$x 1, x 2, x 3, x 4, x 5$ & $510(25.6)$ & 100 & 0 & 0 & 0 & 0 & 0 & \\
\hline$x 1, x 2, x 3, x 4, x 6$ & $-58(23.2)$ & 100 & 0 & 0 & 0 & 0 & 0 & 0 \\
\hline$x 1, x 2, x 3, x 5, x 6$ & $-53(22.8)$ & 100 & 0 & 0 & 0 & 0 & 0 & 0 \\
\hline$x 1, x 2, x 4, x 5, x 6$ & $-39(23.5)$ & 100 & 0 & 0 & 0 & 0 & 0 & 0 \\
\hline$x 1, x 3, x 4, x 5, x 6$ & $-48(23.4)$ & 100 & 0 & 0 & 0 & 0 & 0 & 0 \\
\hline$x 2, x 3, x 4, x 5, x 6$ & $50(24.8)$ & 61 & 39 & 0 & 0 & 0 & 0 & 0 \\
\hline
\end{tabular}

Table 5.26: Summary of model AICc in simulation scenario 4 for the best subset method and for ensemble methods using variable subset sizes of 3,4 , and 5 .

\begin{tabular}{lrr} 
& & Mean (SD) \\
\cline { 3 - 3 } Ensemble Method & \# of subsets & Weighted AICc \\
\hline Size 3 & 20 & $-20(17.8)$ \\
Size 4 & 15 & $-35(19.9)$ \\
Size 5 & 6 & $-27(21.9)$ \\
& & \\
Best Subset & Bandwidth & AICc \\
x1, x3, x6 & 0.1 & $-172(21.8)$ \\
\hline
\end{tabular}


Maps of the best subset and ensemble estimates are given in panels 2 and 3 of Figure 5.19, with the simulated parameter values shown in panel 1 . Both sets of estimates replicate the general spatial pattern in the mixture effect $\mathrm{b} 1$, with central regions of high activity that decrease outwardly in a radial fashion. However, the estimates of the mixture effect appear to be impacted by over smoothing, with a small central cluster of underestimates that transition to overestimates for the remainder of the study region. As shown in Table 5.27, the range of estimated values for b1 is narrowed in comparison to the true simulated range, for both sets of estimates. More specifically, b1, has a simulated range of 0.25 to 0.97 , with estimated ranges of 0.45 to 0.93 and 0.44 to 0.92 , for the best subset and ensemble estimates, respectively.
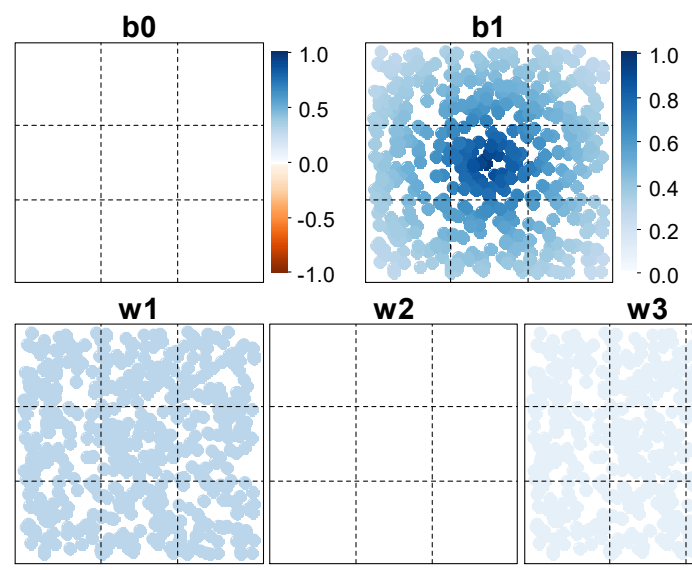

w2 w3

Panel 1. Simulated LWQS parameter values in scenario 4.
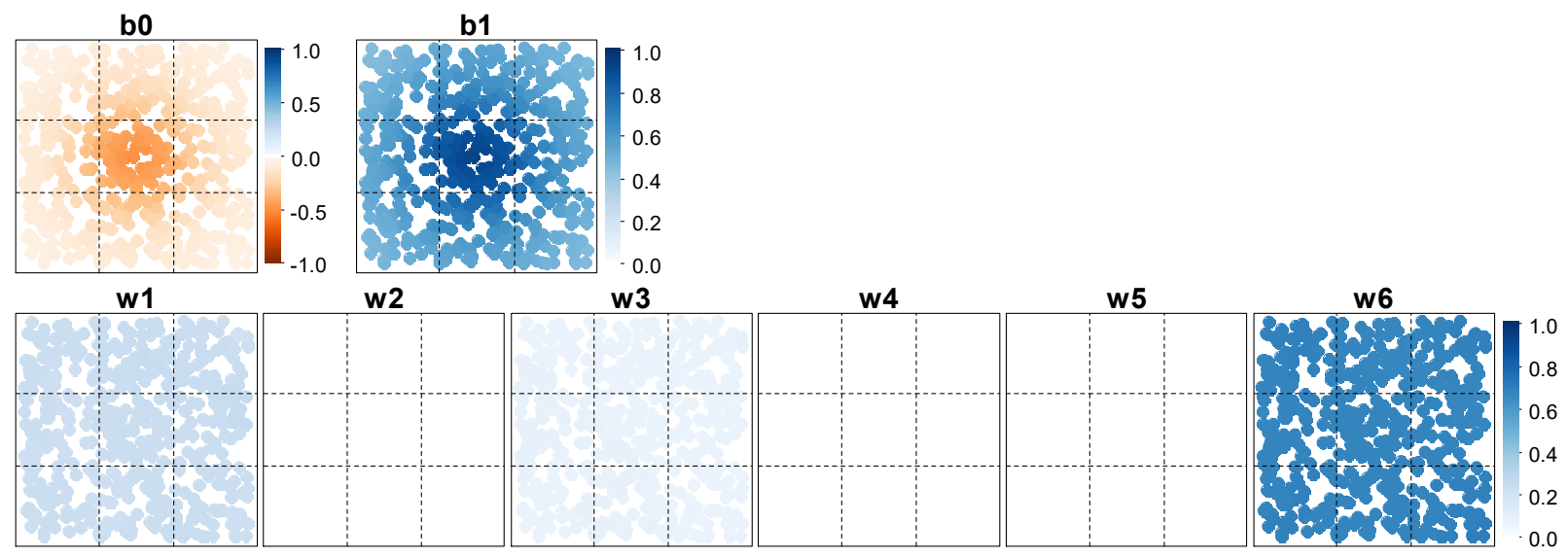

Panel 2. Best subset parameter estimates. 

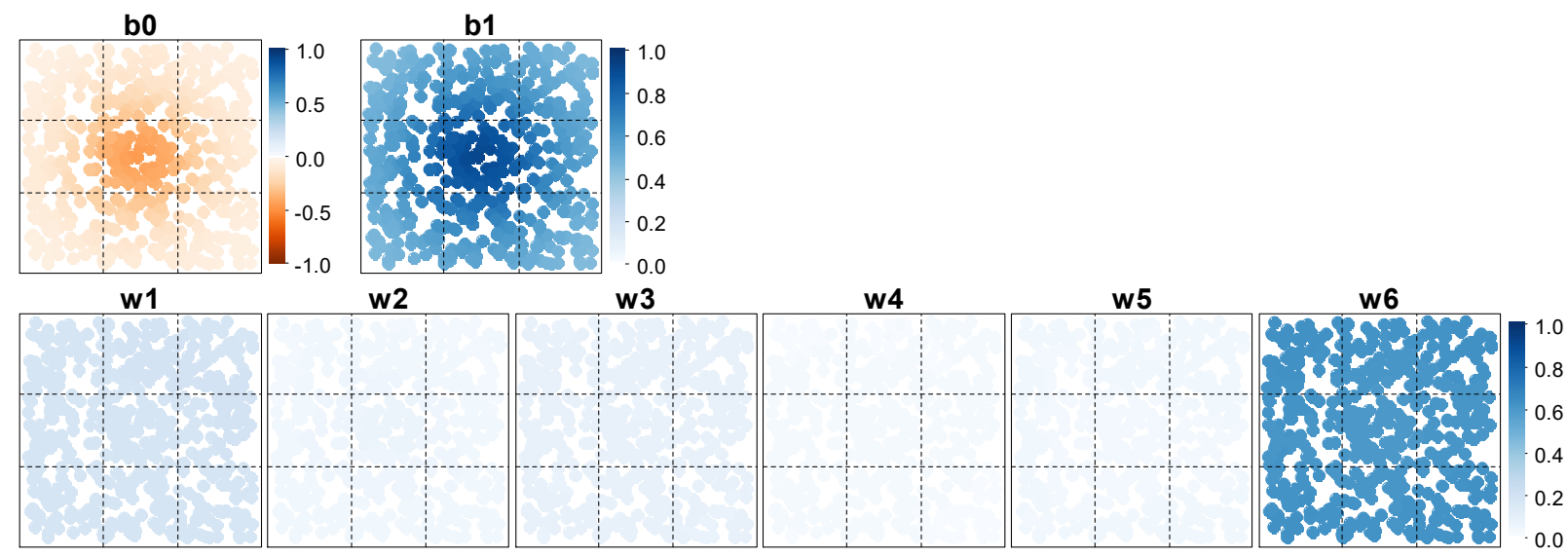

Panel 3. Ensemble estimates from subsets of size 4.

Figure 5.19: Maps of the simulated LWQS parameters (panel 1), best subset estimates (panel 2), and ensemble estimates from subsets of size 4 (panel 3) in simulation scenario 4.

Table 5.27: Summary of the LWQS parameter estimates for simulation scenario 4 using the best subset $^{1}$ and ensemble ${ }^{2}$ methods.

\begin{tabular}{lrrrrrr} 
& & \multicolumn{2}{c}{ Best Subset Estimates } & & \multicolumn{2}{c}{ Ensemble Estimates } \\
\cline { 3 - 4 } \cline { 6 - 7 } & Truth & Mean & Range & & Mean & Range \\
\hline b0 & 0 & -0.17 & $(-0.50,-0.04)$ & & -0.16 & $(-0.46,-0.04)$ \\
w1 & 0.3 & 0.24 & $(0.22,0.25)$ & & 0.19 & $(0.17,0.20)$ \\
w2 & 0 & 0 & $(0,0)$ & & 0.05 & $(0.04,0.07)$ \\
w3 & 0.1 & 0.08 & $(0.07,0.09)$ & & 0.08 & $(0.08,0.09)$ \\
w4 & 0 & 0 & $(0,0)$ & & 0.02 & $(0.02,0.03)$ \\
w5 & 0 & 0 & $(0,0)$ & & 0.04 & $(0.03,0.04)$ \\
w6 & 0.6 & 0.69 & $(0.67,0.70)$ & & 0.62 & $(0.60,0.64)$ \\
& & & & & \\
& Mean (Range) & Mean & Range & & Mean & Range \\
b1 & $0.42(0.25,0.97)$ & 0.62 & $(0.45,0.93)$ & & 0.62 & $(0.44,0.92)$ \\
\hline
\end{tabular}

${ }^{1}$ Best subset estimates pertain to $\mathrm{S}_{\mathrm{b}}=\{\mathrm{x} 1, \mathrm{x} 3, \mathrm{x} 6\}$ with estimated bandwidth value $\gamma=0.1$.

${ }^{2}$ Ensemble estimates are calculated using subsets of size 4.

From Figure 5.19 we also see evidence of collinearity between the intercept, b0, and the mixture effect, b1, in both sets of estimates. More specifically, b0 is underestimated in a pattern that reflects the spatial variation in b1. This is evidenced further when the bias is mapped is mapped over the study region, as shown in Figure 5.20. 


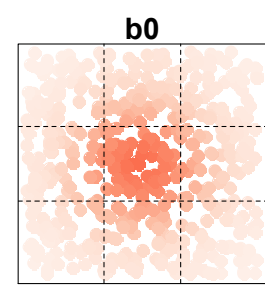

w1
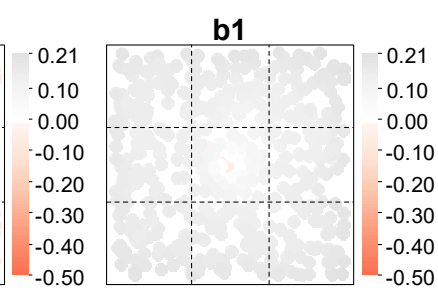

w2 w3

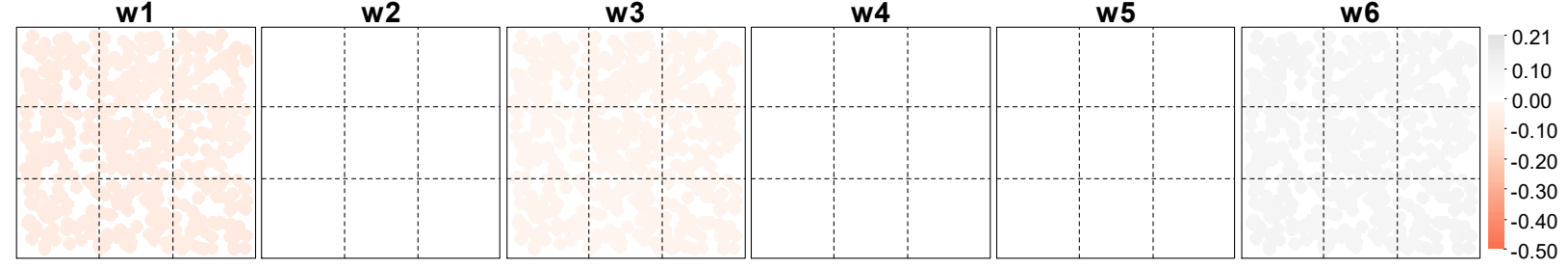

Panel 1. Bias in the best subset parameter estimates.
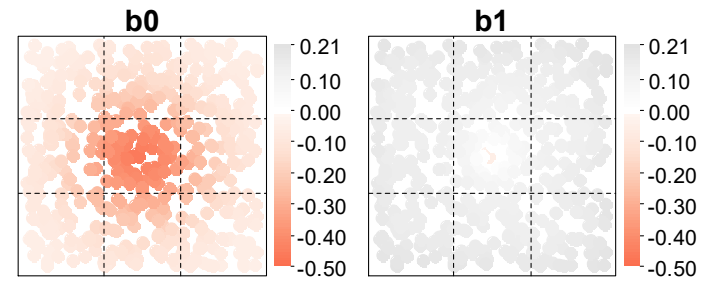

w1

w2

w3

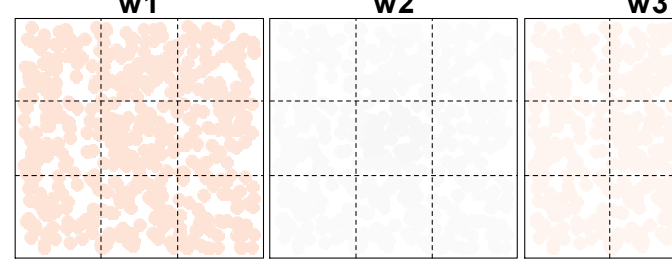

w4

w5

w6
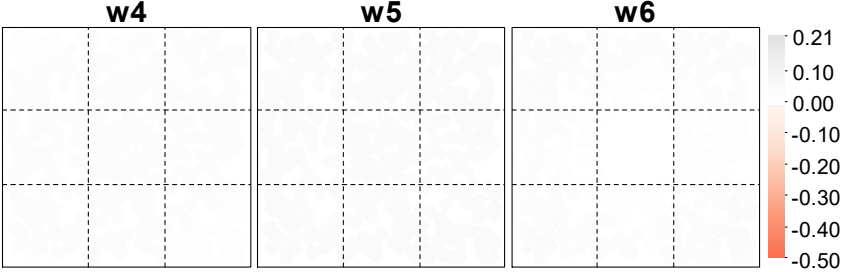

Panel 2. Bias in the ensemble estimates from subsets of size 4.

Figure 5.20: Bias in the best subset estimates (panel 1) and ensemble estimates from subsets of size 4 (panel 2) in simulation scenario 4.

With respect to the weight parameters, we see that no artificial pattern is induced in either set of estimates (Figure 5.19). However, as shown in Figure 5.20, both estimation methods produce underestimates of $\mathrm{w} 1$ and $\mathrm{w} 3$, while overestimating $\mathrm{w} 6$ in the entirety of the study region. On average, the magnitude of bias in the ensemble estimates exceeds that of the best subset estimates for w1, while the reverse is true for estimates of w6 (see Table 5.28). Finally, we note that ensemble method overestimates w2, w4, and w5, while in the best subset model, these parameters are correctly estimated as true zeros by virtue of variable selection. When comparing the estimated weight parameters to a cut-off value of 0.05 , the ensemble estimates correctly 
identify all important exposures (i.e., sensitivity), but also incorrectly identify $\mathrm{x} 2$ as an important exposure in $153(31 \%)$ of the 500 locations.

Table 5.28: Summary of the bias and RMSE in the estimated LWQS parameters for simulation scenario 4 using the best subset ${ }^{1}$ and ensemble ${ }^{2}$ methods.

\begin{tabular}{lrrrrrrrr} 
& \multicolumn{3}{c}{ Best Subset Estimates } & & \multicolumn{3}{c}{ Ensemble Estimates } \\
\cline { 2 - 5 } \cline { 6 - 8 } & Avg. Bias & Bias Range & RMSE & & Avg. Bias & Bias Range & RMSE \\
\hline b0 & -0.17 & $(-0.50,-0.04)$ & 0.21 & & -0.16 & $(-0.46,-0.04)$ & 0.19 \\
b1 & 0.14 & $(-0.04,0.20)$ & 0.15 & & 0.14 & $(-0.05,0.20)$ & 0.14 \\
w1 & -0.06 & $(-0.08,-0.05)$ & 0.06 & & -0.11 & $(-0.13,-0.10)$ & 0.11 \\
w2 & 0 & $(0,0)$ & 0 & & 0.05 & $(0.04,0.07)$ & 0.05 \\
w3 & -0.02 & $(-0.03,-0.01)$ & 0.02 & & -0.02 & $(-0.02,-0.01)$ & 0.02 \\
w4 & 0 & $(0,0)$ & 0 & & 0.02 & $(0.02,0.03)$ & 0.02 \\
w5 & 0 & $(0,0)$ & 0 & & 0.04 & $(0.03,0.04)$ & 0.04 \\
w6 & 0.09 & $(0.07,0.10)$ & 0.09 & & 0.02 & $(0.00,0.04)$ & 0.02 \\
\hline
\end{tabular}

${ }^{1}$ Best subset estimates pertain to $S_{b}=\{x 1, x 3, x 6\}$ with estimated bandwidth value $\gamma=0.1$.

${ }^{2}$ Ensemble estimates are calculated using subsets of size 4 .

The best subset and ensemble solutions were comparable in performance when estimating the intercept, $b 0$, and mixture effect, b1. Both were able to replicate the general spatial pattern in b1, but collinearity between the intercept and mixture effect limit the interpretability of b0. While interpretation of the intercept is cautioned, inference can be made regarding the mixture effect in both estimation methods. When considering variable selection and estimation of the weight parameters, the best subset solutions were superior in the correct identification of inactive exposures.

\section{Scenario 5}

Scenario 5 extends the simulation settings in scenario 4 to include spatially dependent weight parameters in addition to the spatially varying exposures and mixture effect. As in the previous scenario, concentrations for $\mathrm{x} 2, \mathrm{x} 4$, and $\mathrm{x} 6$ decrease radially from the center of the study region. The mixture effect is strongest in the center of the study area and also decreases outwardly in a radial pattern. Scenario 6 introduces spatial dependency in the weight parameters by allowing w6 
to decrease radially in a pattern similar to that of $\mathrm{x} 6$. The remaining weight is randomly allocated between w1 and w3 in proportions ranging from 0.3 to 0.7 .

Model goodness-of-fit and choice of bandwidth parameter are summarized for all subset models in Table 5.29. The best subset was again correctly chosen as $S_{b}=\{x 1, x 3, x 6\}$, with the minimum average AICc across all subsets models. Consistent with the former scenario, the estimated bandwidth parameters are largely limited to the two smallest candidate values $(\gamma=0.1$, and 0.2$)$, with the exception of the subset containing exposures $\mathrm{x} 2, \mathrm{x} 4$, and $\mathrm{x} 6$. The ensemble estimates calculated using subsets of size 4 are again selected for presentation, with the minimum weighted AICc across all candidate subset sizes (Table 5.30). Finally, AICc for the best subset is again far lower than the weighted AICc for any subset size, suggesting superior goodness-of-fit for the best subset model.

Table 5.29: Summary of model AICc by variable subset and the corresponding number of times each candidate bandwidth value $\gamma$ was chosen over the 100 simulated data sets for scenario 5 .

\begin{tabular}{|c|c|c|c|c|c|c|c|c|c|}
\hline \multirow[b]{3}{*}{ Subset } & \multirow[b]{2}{*}{$\mathrm{AICc}$} & \multicolumn{8}{|c|}{ \# Times Selected } \\
\hline & & \multicolumn{8}{|c|}{ Bandwidth Value $\gamma$} \\
\hline & Mean (SD) & 0.1 & 0.2 & 0.3 & 0.5 & 0.6 & 0.8 & 1.0 & 1.4 \\
\hline $\mathrm{x} 1, \mathrm{x} 2, \mathrm{x} 3$ & $256(23.7)$ & 100 & 0 & 0 & 0 & 0 & 0 & 0 & 0 \\
\hline $\mathrm{x} 1, \mathrm{x} 2, \mathrm{x} 4$ & $304(22.9)$ & 100 & 0 & 0 & 0 & 0 & 0 & 0 & 0 \\
\hline$x 1, x 2, x 5$ & $305(23.8)$ & 100 & 0 & 0 & 0 & 0 & 0 & 0 & 0 \\
\hline$x 1, x 2, x 6$ & $17(21.8)$ & 100 & 0 & 0 & 0 & 0 & 0 & 0 & 0 \\
\hline $\mathrm{x} 1, \mathrm{x} 3, \mathrm{x} 4$ & $285(20.7)$ & 100 & 0 & 0 & 0 & 0 & 0 & 0 & 0 \\
\hline$x 1, x 3, x 5$ & $293(20.0)$ & 100 & 0 & 0 & 0 & 0 & 0 & 0 & 0 \\
\hline $\mathbf{x 1 ,} \times 3, \times 6$ & $-83(18.8)$ & 100 & 0 & 0 & 0 & 0 & 0 & 0 & 0 \\
\hline$x 1, x 4, x 5$ & $332(22.7)$ & 100 & 0 & 0 & 0 & 0 & 0 & 0 & 0 \\
\hline$x 1, x 4, x 6$ & $21(21.8)$ & 100 & 0 & 0 & 0 & 0 & 0 & 0 & 0 \\
\hline$x 1, x 5, x 6$ & $3(22.5)$ & 100 & 0 & 0 & 0 & 0 & 0 & 0 & 0 \\
\hline$x 2, x 3, x 4$ & $308(25.5)$ & 100 & 0 & 0 & 0 & 0 & 0 & 0 & 0 \\
\hline$x 2, x 3, x 5$ & $305(26.5)$ & 100 & 0 & 0 & 0 & 0 & 0 & 0 & 0 \\
\hline$x 2, x 3, x 6$ & $21(21.9)$ & 100 & 0 & 0 & 0 & 0 & 0 & 0 & 0 \\
\hline$x 2, x 4, x 5$ & $429(29.0)$ & 100 & 0 & 0 & 0 & 0 & 0 & 0 & 0 \\
\hline$x 2, x 4, x 6$ & $536(21.0)$ & 0 & 20 & 46 & 22 & 6 & 3 & 3 & 0 \\
\hline$x 2, x 5, x 6$ & $222(29.4)$ & 100 & 0 & 0 & 0 & 0 & 0 & 0 & 0 \\
\hline
\end{tabular}




\begin{tabular}{lrrrrrrrrr} 
x3, x4, x5 & $331(22.9)$ & 100 & 0 & 0 & 0 & 0 & 0 & 0 & 0 \\
x3, x4, x6 & $26(22.8)$ & 100 & 0 & 0 & 0 & 0 & 0 & 0 & 0 \\
x3, x5, x6 & $2(21.2)$ & 100 & 0 & 0 & 0 & 0 & 0 & 0 & 0 \\
x4, x5, x6 & $227(29.3)$ & 100 & 0 & 0 & 0 & 0 & 0 & 0 & 0 \\
& & & & & & & & & \\
x1, x2, x3, x4 & $304(24.1)$ & 100 & 0 & 0 & 0 & 0 & 0 & 0 & 0 \\
x1, x2, x3, x5 & $321(25.6)$ & 100 & 0 & 0 & 0 & 0 & 0 & 0 & 0 \\
x1, x2, x3, x6 & $-30(19.6)$ & 100 & 0 & 0 & 0 & 0 & 0 & 0 & 0 \\
x1, x2, x4, x5 & $352(24.2)$ & 100 & 0 & 0 & 0 & 0 & 0 & 0 & 0 \\
x1, x2, x4, x6 & $75(22.6)$ & 100 & 0 & 0 & 0 & 0 & 0 & 0 & 0 \\
x1, x2, x5, x6 & $56(22.7)$ & 100 & 0 & 0 & 0 & 0 & 0 & 0 & 0 \\
x1, x3, x4, x5 & $351(22.5)$ & 100 & 0 & 0 & 0 & 0 & 0 & 0 & 0 \\
x1, x3, x4, x6 & $-25(20.1)$ & 100 & 0 & 0 & 0 & 0 & 0 & 0 & 0 \\
x1, x3, x5, x6 & $-19(19.5)$ & 100 & 0 & 0 & 0 & 0 & 0 & 0 & 0 \\
x1, x4, x5, x6 & $61(23.1)$ & 100 & 0 & 0 & 0 & 0 & 0 & 0 & 0 \\
x2, x3, x4, x5 & $352(27.5)$ & 100 & 0 & 0 & 0 & 0 & 0 & 0 & 0 \\
x2, x3, x4, x6 & $79(22.9)$ & 100 & 0 & 0 & 0 & 0 & 0 & 0 & 0 \\
x2, x3, x5, x6 & $55(22.0)$ & 100 & 0 & 0 & 0 & 0 & 0 & 0 & 0 \\
x2, x4, x5, x6 & $268(27.2)$ & 29 & 71 & 0 & 0 & 0 & 0 & 0 & 0 \\
x3, x4, x5, x6 & $60(22.6)$ & 100 & 0 & 0 & 0 & 0 & 0 & 0 & 0 \\
& & & & & & & & & \\
x1, x2, x3, x4, x5 & $369(25.9)$ & 100 & 0 & 0 & 0 & 0 & 0 & 0 & 0 \\
x1, x2, x3, x4, x6 & $30(21.2)$ & 100 & 0 & 0 & 0 & 0 & 0 & 0 & 0 \\
x1, x2, x3, x5, x6 & $34(20.5)$ & 100 & 0 & 0 & 0 & 0 & 0 & 0 & 0 \\
x1, x2, x4, x5, x6 & $115(23.7)$ & 99 & 1 & 0 & 0 & 0 & 0 & 0 & 0 \\
x1, x3, x4, x5, x6 & $39(21.0)$ & 100 & 0 & 0 & 0 & 0 & 0 & 0 & 0 \\
x2, x3, x4, x5, x6 & $114(23.5)$ & 99 & 1 & 0 & 0 & 0 & 0 & 0 & 0 \\
\hline & & & & & & & &
\end{tabular}

Table 5.30: Summary of model AICc in simulation scenario 5 for the best subset method and for ensemble methods using variable subset sizes of 3,4 , and 5 .

\begin{tabular}{lrr} 
& & Mean (SD) \\
\cline { 3 - 3 } Ensemble Method & \# of subsets & Weighted AICc \\
\hline Size 3 & 20 & $62(16.0)$ \\
Size 4 & 15 & $48(17.6)$ \\
Size 5 & 6 & $61(19.7)$ \\
& & \\
Best Subset & Bandwidth & AICc \\
x1, x3, x6 & 0.1 & $-83(18.8)$ \\
\hline
\end{tabular}

Maps of the best subset and ensemble estimates are given in panels 2 and 3 of Figure 5.21, with the simulated parameter values shown in panel 1. Both sets of estimates replicate the general spatial pattern in the mixture effect, b1, but are again impacted by over-smoothing. The estimates 
of $\mathrm{b} 1$ correctly detect the central region of high activity, but fail to decay as rapidly as the true simulated values. Additionally, the estimates of b0 again reflect the spatial pattern in b1, with a central region of underestimation that decreases in magnitude when approaching the peripheral. Both the best subset and ensemble methods produce overly-smooth estimates of w1 and w3, demonstrating difficulty in replicating the local (point to point) variation in the true simulated values. With respect to w6, we see that while both estimates are impacted by over-smoothing, the ensemble estimates have greater difficulty in distinguishing the central region of high activity. This is shown more clearly in Figure 5.22, where bias is mapped over the study area for each set of estimates. Here, the central region of underestimation in the ensemble estimates of w6 is clearly visualized in red (panel 2).
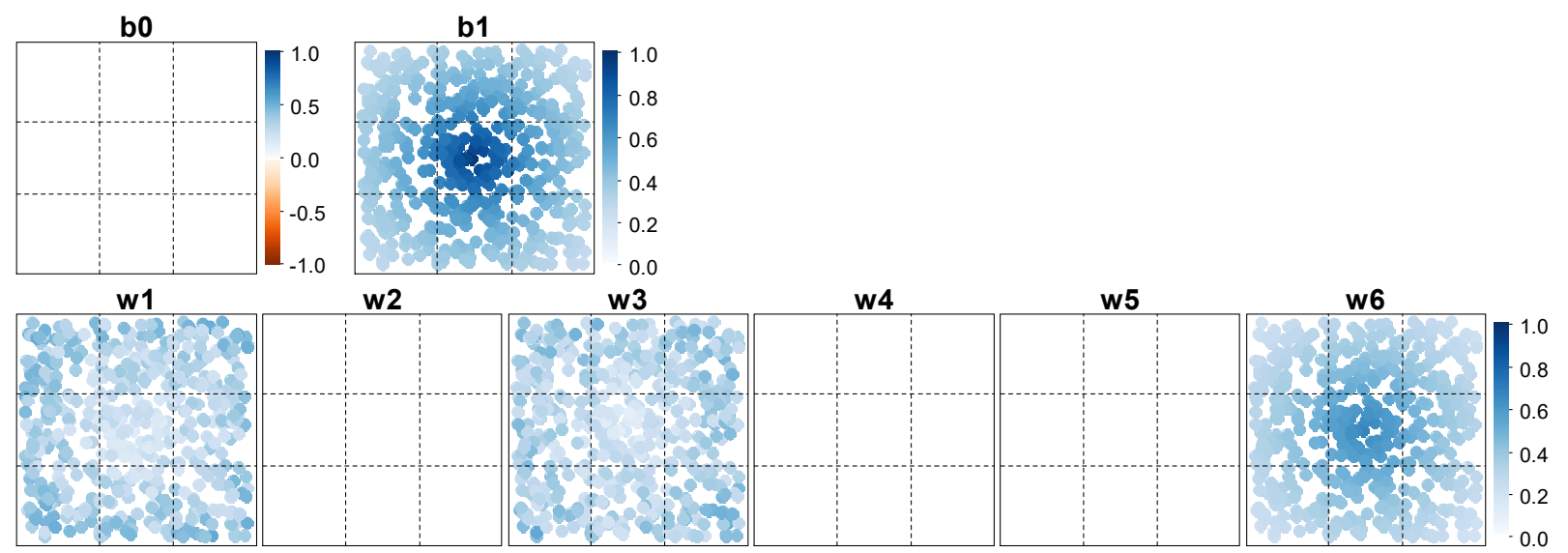

Panel 1. Simulated LWQS parameter values in scenario 5. 


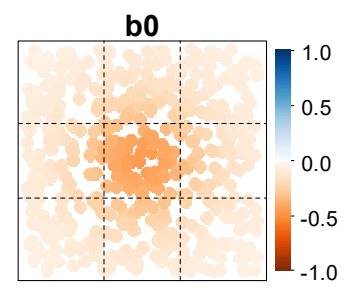

w1

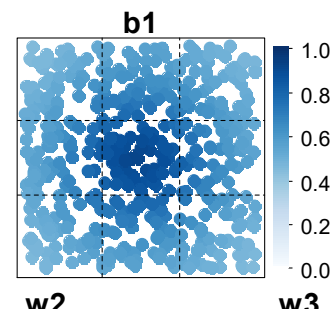

w3

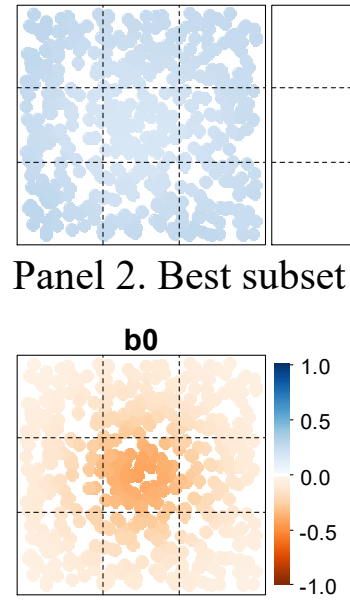

w2

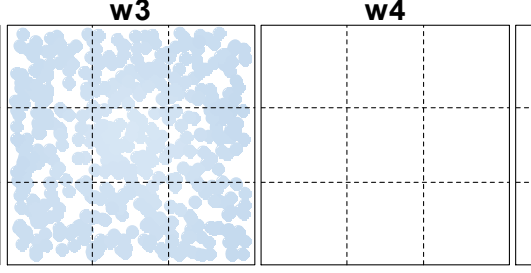

w5

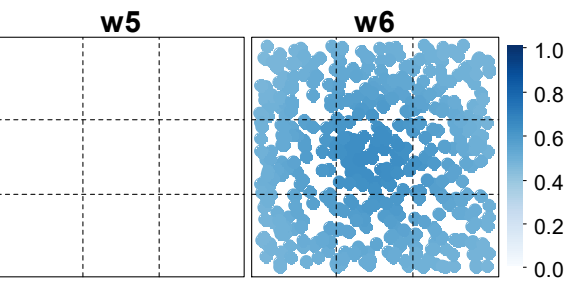

w1

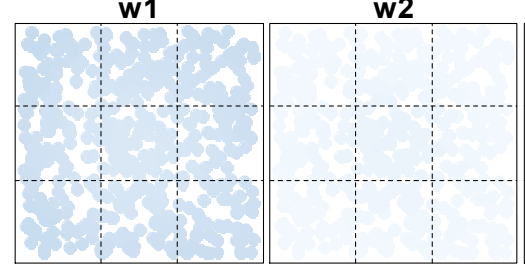

b1

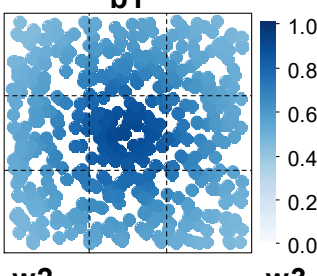

w3

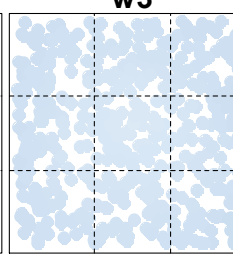

w4

w4 w5

w5 w6

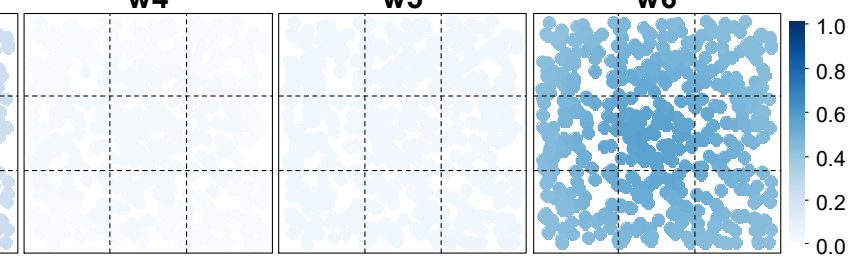

Panel 3. Ensemble estimates from subsets of size 4.

Figure 5.21: Maps of the simulated LWQS parameters (panel 1), best subset estimates (panel 2), and ensemble estimates from subsets of size 4 (panel 3) in simulation scenario 5. 


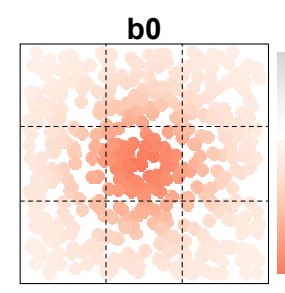

w1

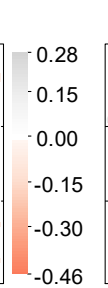

w2 b1

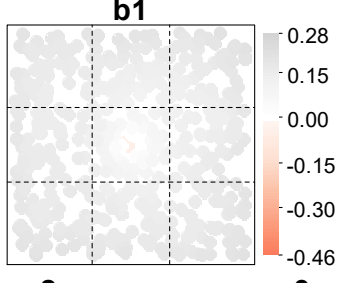

w3

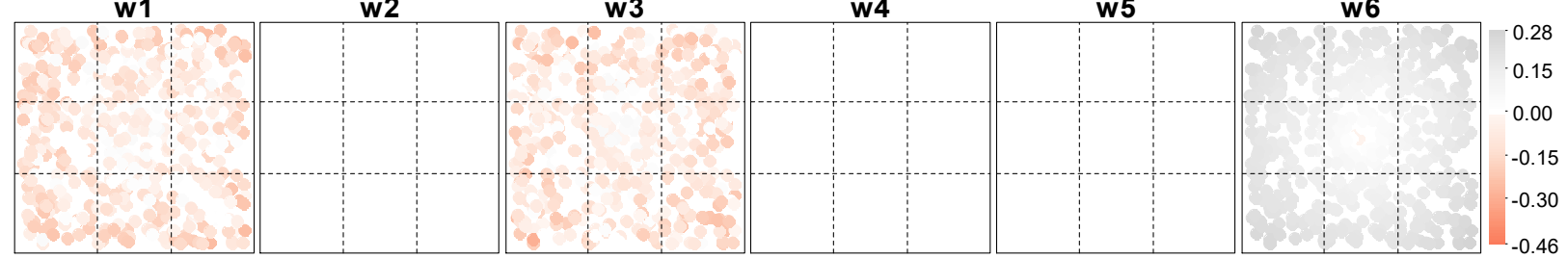

Panel 1. Bias in the best subset parameter estimates.
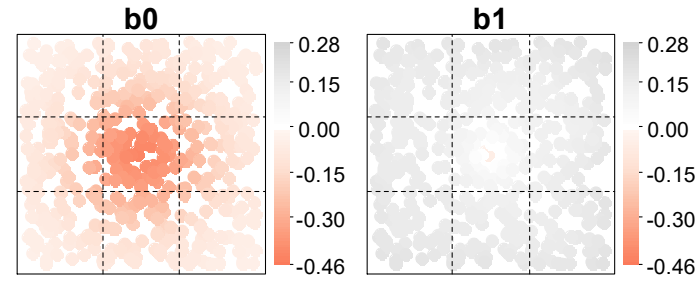

w1

w2

w3
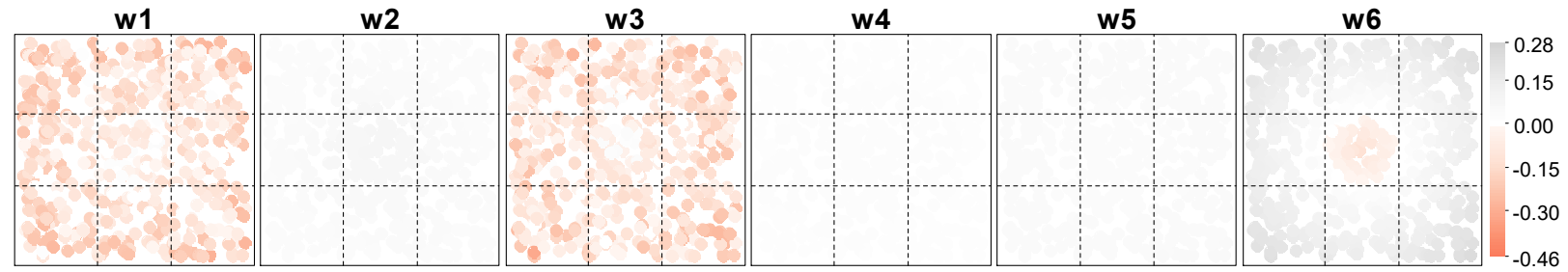

Panel 2. Bias in the ensemble estimates from subsets of size 4.

Figure 5.22: Bias in the best subset estimates (panel 1) and ensemble estimates from subsets of size 4 (panel 2) in simulation scenario 5.

Figure 5.22 also elucidates the general overestimation of $\mathrm{b} 1$ and w6, in comparison to the general underestimation of $\mathrm{w} 1$ and $\mathrm{w} 3$, for both sets of estimates. The range of estimated values is shown in comparison to the true simulated range for each of the spatially dependent parameters in Table 5.31. Here we see a notable overestimation of the lower bound in b1 and w6, and notable underestimation of the upper bound in w1 and w3, for both sets of estimates. With that said, we deemphasize interpretation of average values calculated over the study area for the spatially dependent parameters. The locations of estimated values and bias are more telling with respect to a method's ability to detect spatial features. Therefore, Tables $\mathbf{5 . 3 1}$ and 
5.32 should be interpreted in the presence of the corresponding maps in Figures 5.21 and 5.22, respectively.

Table 5.31: Summary of the LWQS parameter estimates for simulation scenario 5 using the best subset $^{1}$ and ensemble ${ }^{2}$ methods.

\begin{tabular}{rrrrr} 
& & Best Subset Estimates & & Ensemble Estimates \\
\cline { 5 - 5 } & & Mean (Range) & Mean (Range) \\
\hline b0 & 0 & $-0.17(-0.46,-0.04)$ & & $-0.16(-0.43,-0.04)$ \\
w2 & 0 & $0(0,0)$ & & $0.05(0.03,0.07)$ \\
w4 & 0 & $0(0,0)$ & $0.02(0.01,0.03)$ \\
w5 & & $0(0,0)$ & $0.04(0.03,0.05)$ \\
& & & \\
& Mean (Range) & Mean (Range) & Mean (Range) \\
b1 & $0.42(0.25,0.97)$ & $0.63(0.45,0.92)$ & & $0.62(0.45,0.92)$ \\
w1 & $0.31(0.12,0.53)$ & $0.23(0.16,0.29)$ & & $0.21(0.15,0.25)$ \\
w3 & $0.30(0.11,0.53)$ & $0.23(0.17,0.27)$ & $0.20(0.15,0.24)$ \\
w6 & $0.39(0.29,0.69)$ & $0.54(0.47,0.66)$ & $0.48(0.42,0.56)$ \\
\hline
\end{tabular}

${ }^{1}$ Best subset estimates pertain to $S_{b}=\{x 1, x 3, x 6\}$ with estimated bandwidth value $\gamma=0.1$.

${ }^{2}$ Ensemble estimates are calculated using subsets of size 4 .

Table 5.32: Summary of the bias and RMSE in the estimated LWQS parameters for simulation scenario 5 using the best subset ${ }^{1}$ and ensemble ${ }^{2}$ methods.

\begin{tabular}{lrrrrrrrr} 
& \multicolumn{3}{c}{ Best Subset Estimates } & & \multicolumn{3}{c}{ Ensemble Estimates } \\
\cline { 2 - 5 } \cline { 6 - 8 } & Avg. Bias & Bias Range & RMSE & & Avg. Bias & Bias Range & RMSE \\
\hline b0 & -0.17 & $(-0.46,-0.04)$ & 0.20 & & -0.16 & $(-0.43,-0.04)$ & 0.18 \\
b1 & 0.15 & $(-0.05,0.21)$ & 0.15 & & 0.14 & $(-0.05,0.20)$ & 0.15 \\
w1 & -0.08 & $(-0.26,0.06)$ & 0.11 & & -0.10 & $(-0.29,0.04)$ & 0.13 \\
w2 & 0 & $(0,0)$ & 0 & & 0.05 & $(0.03,0.07)$ & 0.05 \\
w3 & -0.08 & $(-0.30,0.06)$ & 0.11 & & -0.10 & $(-0.31,0.04)$ & 0.13 \\
w4 & 0 & $(0,0)$ & 0 & & 0.02 & $(0.01,0.03)$ & 0.02 \\
w5 & 0 & $(0,0)$ & 0 & & 0.04 & $(0.03,0.05)$ & 0.04 \\
w6 & 0.16 & $(-0.03,0.28)$ & 0.17 & & 0.10 & $(-0.13,0.23)$ & 0.12 \\
\hline
\end{tabular}

${ }^{1}$ Best subset estimates pertain to $S_{b}=\{x 1, x 3, x 6\}$ with estimated bandwidth value $\gamma=0.1$.

${ }^{2}$ Ensemble estimates are calculated using subsets of size 4 .

Finally, when using a cut-off value of 0.05 for identification of important exposures, the ensemble estimates perform well in terms of sensitivity, correctly selecting $\mathrm{x} 1, \mathrm{x} 3$, and $\mathrm{x} 6$ as important at all locations. However, in terms of specificity, the ensemble estimates incorrectly 
identify $\mathrm{x} 2$ as important in $145(29 \%)$ of the 500 locations, while $\mathrm{x} 5$ is incorrectly identified as important in $9(2 \%)$ of the total locations. The misclassification of $\mathrm{x} 2$ by the ensemble estimates in both scenario 4 and scenario 5 may be a consequence of the exposure's relationship with the heavily weighted exposure $\mathrm{x} 6$.

Scenario 5 has shown that LWQS is capable of identifying spatially dependent mixture effects and parameter weights in the presence of correlated spatially dependent exposures. The best subset and ensemble solutions were both able to replicate the general spatial pattern in the mixture effect, b1. Further, while neither set of solutions were immune to the effects of oversmoothing, the best subset solutions were superior in recognizing the spatial pattern in the parameter weights. As in scenario 4, estimates of the intercept, b0, demonstrated collinearity with the mixture effect, limiting the interpretability of the intercept in the local models. Finally, both sets of solutions were able to correctly identify all important exposures, but the best subset solutions provide a more decisive method of variable selection, and outperformed the ensemble solutions in the identification of unimportant exposures (i.e., specificity).

\subsection{Conclusions}

In this simulation study we considered five scenarios of increasing complexity with respect to spatial dependency. We also systematically increased collinearity in the simulated exposure data and demonstrated that LWQS model performance is generally robust to the extreme effects of collinearity. Both estimation methods (best subset and ensemble) were able to accurately identify spatial patterns in the mixture effect. However the best subset solutions improved on the accuracy of the estimated parameter (i.e., chemical importance) weights when compared to the ensemble approach. While bias was still present in the best subset solutions due to oversmoothing, no spatial artifacts were present in the estimates of the mixture effect or weight 
parameters. Additionally, the appropriate spatial patterns were consistently intact. In the final two scenarios, we saw collinearity in the estimates of the intercept and mixture effect for both sets of solutions suggesting identifiability issues with $b 0$ and $b 1$ when spatial dependency is introduced in the exposures. However, the collinearity only impacted the interpretability of the intercept, and we are still able to make inference regarding the parameter of interest, b1. We note also that that the inclusion of covariates in the LWQS model may help to diffuse the degree to which the intercept tracks the mixture effect. Encouragingly, we saw very little tendency for the underestimation of the mixture effect, and no evidence of incorrect classification of harmful exposures. The best subset solutions also consistently selected an index containing only the three important exposures, demonstrating perfect specificity in addition to perfect sensitivity in all scenarios considered in this study. 


\title{
Chapter 6
}

\author{
Conclusions and Future Work
}

\subsection{Conclusions}

In this work we demonstrated the importance of examining the relationship between chemical mixtures and disease risk, and focused on the development of analysis strategies that effectively accommodate complex and spatially varying exposure patterns. Through simulation studies, we illustrated the limited applicability of existing methods for the analysis of spatially varying effects, and motivated the development of new methodology that effectively models spatial variation in individual and composite exposure effects in environmental health models.

In response, we developed local weighted quantile sum (LWQS) regression - a spatial extension of the WQS method that accommodates spatially varying effects of chemical mixtures. The performance of LWQS was assessed in a simulation study that considered several environmentally relevant scenarios of increasing complexity with respect to spatial dependency and correlation among simulated exposures. Results showed that LWQS is capable of spatially accurate variable selection and signal detection when modeling the associations of multiple exposures and a continuous response. LWQS was largely unhindered by the presence of complex correlations, and was able to consistently distinguish between important exposures and those unrelated to the response. The LWQS estimates replicated spatially varying effects when present, 
and no spatial artifacts were present in estimates of the mixture effect or exposure weights, when the true underlying process was constant.

\subsection{Future Work}

The LWQS model and simulation study presented in this work was limited to the application of exposure data with a continuous response variable. Future work will extend the LWQS model to a more generalized version that accommodates multiple types of response data. Simulation studies will evaluate the performance of LWQS using non-continuous outcomes, with the intent of applying the LWQS method to the NCI-SEER case-control study of NHL.

In chapter 5 we presented two approaches to the estimation of the final LWQS weights based on variable subspaces. The first selected the best subset over a range of candidate subset sizes according to model goodness-of-fit. The second used an ensemble strategy to create local composite estimates across variable subsets of the same size. Because our simulation study was limited to six exposures, we were able to estimate models for all subsets at all subset sizes. Future work will consider the use of random subsets in larger sets of exposures, and/or the development of a search algorithm to aid in the identification of the best subset solutions, and selection of subset size in the ensemble approach. Incorporation of random subsets (as opposed to exhaustive estimation of all subset solutions) will be particularly important in higher dimensional scenarios. While our current methodology incorporates a variable subspace approach as a method of diversity generation and variable selection, future work will explore other forms of diversity generation in the estimation of final solutions, including random subsampling of the observations. 
In both estimation methods (best subset and ensemble), AICc was selected as the measure of signal with the understanding that WQS regression is typically used for variable selection over prediction. However, it would be interesting to compare the performance of both estimates using alternative selection criteria and/or alternative signal functions in identification of the best subset and calculation of the composite (ensemble) weights. In higher dimensional settings, ensemble methods may have the advantage of estimating parameters in subsets of varying degrees of spatial dependency, allowing the composite estimates to better reflect the true spatial variation of model effects. Future work will evaluate if the ensemble subset approach has better relative performance with alternative signal functions and larger chemical mixtures of highly correlated components.

Examination of the trend in AICc across subset models can provide valuable insight in terms of variable importance (i.e., recognition that exclusion/inclusion of particular exposure results in marked changes in model AICc). Similarly, trend in bandwidth parameter selection regarding inclusion/exclusion of variables is indicative of the degree of spatial dependency introduced by an exposure in the model. Changes in the measure of signal across subsets and bandwidth parameters is a potential avenue into the development of search algorithms for choice of subsets and estimation of the bandwidth parameter.

Finally, in simulation scenario 2 and 3, LWQS demonstrated difficulty in detecting the linear divisions between risk regions - likely resulting from use of the exponential kernel function. Future implementations of LWQS should consider the use of triangulation methods to improve the detection of spatial features with linear boundaries. 


\section{List of References}




\section{List of References}

Bithell JF, Dutton SJ, Draper GJ, Neary NM. 1994. Distribution of childhood leukaemias and non-Hodgkin's lymphomas near nuclear installations in England and Wales. BMJ 309(6953):501-505.

Breiman L, Friedman J, Olshen R, Stone C. Classification and regression trees. Wadsworth \& Brooks/Cole Advanced Books \& Software; 1984.

Carrico C. Characterization of a weighted quantile score approach for highly correlated data in risk analysis scenarios. VCU Theses and Dissertations: Biostatistics, Virginia Commonwealth University; 2013.

Carrico C, Gennings C, Wheeler DC, Factor-Litvak P. 2014. Characterization of a weighted quantile sum regression for highly correlated data in a risk analysis setting. J Agr Biol Envir St.

Cleveland W S, 1979, "Robust locally-weighted regression and smoothing scatterplots" Journal of the American Statistical Association 74829 - 836.

Colt JS, Davis S, Severson RK, Lynch CF, Cozen W, Camann D, et al. 2006. Residential Insecticide Use and Risk of Non-Hodgkin's Lymphoma. Cancer Epidemiol Biomarkers Prev 15(2):251-257.

Colt JS, Lubin J, Camann D, Davis S, Cerhan J, Severson RK, et al. 2004. Comparison of pesticide levels in carpet dust and self-reported pest treatment practices in four US sites. $\mathrm{J}$ Expo Anal Env Epid 14:74-83.

Colt JS, Severson RK, Lubin J, Rothman N, Camann D, Davis S, et al. 2005. Organochlorines in carpet dust and non-Hodgkin lymphoma. Epidemiology 16(4):516-525.

Czarnota J, Gennings C, Colt JS, De Roos AJ, Cerhan JR, Severson RK, Hartge P, Ward MH, Wheeler D. 2015. Analysis of environmental chemical mixtures and non-Hodgkin lymphoma risk in the NCI-SEER NHL study. Environmental Health Perspectives, 123:965-970, http://dx.doi.org/10.1289/ehp.1408630.6.

Czarnota J, Gennings C, Wheeler D. 2015 . Assessment of weighted quantile sum regression for modeling chemical mixtures and cancer risk. Cancer Informatics, 14(Suppl 2): 159-171.

Czarnota J, Wheeler D. 2015. WQS: Weighted quantile sum regression. R package. URL: https://cran.fhcrc.org/web/packages/wqs/index.html. 
Czarnota J, Wheeler D, Gennings C. $2015^{\mathrm{b}}$. Evaluating geographically weighted regression models for environmental chemical risk analysis. Cancer Informatics, 14(Suppl 2): 117-127. 5

De Roos AJ, Davis S, Colt JS, Blair A, Airola M, Severson RK, et al. 2010. Residential proximity to industrial facilities and risk of non-Hodgkin lymphoma. Environ Res 110(1):70-78.

De Roos AJ, Hartge P, Lubin JH, Colt JS, Davis S, Cerhan JR, et al. 2005. Persistent organochlorine chemicals in plasma and risk of non-Hodgkin's lymphoma. Cancer Res 65(23):11214-11226.

DellaValle C, Wheeler D, Deziel N, et al. Environmental determinants of polychlorinated biphenyl concentrations in residential carpet dust. Environmental Science \& Technology. 2013;47(18):10405-10414.

Dreiher J, Novack V, Barachana M, Yerushalmi R, Lugassy G, Shpilberg O. 2005. NonHodgkin's lympohma and residential proximity to toxic industrial waste in southern Israel. Haematologica 90(12):1709-1710.

Engel LS, Laden F, Andersen A, Strickland PT, Blair A, Needham LL, et al. 2007a.

Polychlorinated biphenyl levels in peripheral blood and non-Hodgkin's lymphoma: A report from three cohorts. Cancer Res 67(11):5545-5552.

Engel LS, Lan Q, Rothman N. 2007b. Polychlorinated biphenyls and non-Hodgkin lymphoma. Cancer Epidemiol Biomarkers Prev 16(3):373-376.

Everett CJ, Mainous AG III, Frithsen IL, Player MS, Matheson EM. 2008. Association of polychlorinated biphenyls with hypertension in the 1999-2002 National Health and Nutrition Examination Survey. Environ Res 108(1):94-97.

Floret N, Mauny F, Challier B, Arveux P, Cahn JY, Viel JF. 2003. Dioxin emissions from a solid waste incinerator and risk of non-Hodgkin lymphoma. Epidemiology 14(4):392-398.

Fotheringham A, Brunsdon C, Charlton M, 2002. Geographically Weighted Regression: The Analysis of Spatially Varying Relationships (Wiley, Chichester).

Franchini M, Rial M, Buiatti E, Bianchi F. 2004. Health effects of exposure to waste incinerator emissions: a review of epidemiological studies. Ann Ist Super Sanità 40(1):101-115.

Friedman J, Hastie T, Tibshirani R. Regularization paths for generalized linear models via coordinate descent. Journal of Statistical Software. 2010;33(1):1-22.

Goldberg MS, Siemiatyck J, DeWar R, Désy M, Riberdy H. 1999. Risk of developing cancer relative to living near a municipal solid waste landfill site in Montreal, Quebec, Canada. Arch Environ Health 54(4):291-296.

Hartge P, Colt JS, Severson RK, Cerhan JR, Cozen W, Camann D, et al. 2005. Residential herbicide use and risk of non-Hodgkin lymphoma. Cancer Epidemiol Biomarkers Prev 14:934937.

Hartge P, Wang S, Bracci P, Devesa S, Holly E. 2006. Non-Hodgkin lymphoma. In: Cancer Epidemiology and Prevention, Vol. 3rd edition, (Schottenfeld D, Fraumeni J, Jr., eds). New York:Oxford University Press, 898-918. 
Hastie T, Tibshirani R, Friedman J, 2001 The Elements of Statistical Learning: Data Mining, Inference, and Prediction (Springer-Verlag, New York).

Ioannidis JP, Loy EY, Poulton R, Chia KS. 2009. Researching genetic versus nongenetic determinants of disease: A comparison and proposed unification. Sci Transl Med 1(7):7ps8.

Khun M, Johnson K, 2013. Applied Predictive Modeling (Springer, New York).

Knobeloch L, Turyk M, Imm P, Anderson H. 2012. Polychlorinated biphenyls in vacuum dust and blood of residents in 20 Wisconsin households. Chemosphere 86(7):735-740.

Lanphear BP, Matte TD, Rogers J, Clickner RP, Dietz B, Bornschein RL, et al. 1998. The contribution of lead-contaminated house dust and residential soil to children's blood lead levels. Environmental Research, Section A 79(1):51-68.

Loader C, 1999 Local Regression and Likelihood (Springer, New York).

Lubin JH, Colt JS, Camann D, Davis S, Cerhan JR, Severson R, et al. 2004. Epidemiologic evaluation of measurement data in the presence of detection limits. Environ Health Perspect 112(17):1691-1696.

Morton LM, Wang SS, Cozen W, Linet MS, Chatterjee N, Davis S, et al. 2008. Etiologic heterogeneity among non-Hodgkin lymphoma subtypes. Blood 112(13):5150-5160.

Páez A, Farber S, Wheeler D. 2011. A simulation-based study of geographically weighted regression as a method for investigating spatially varying relationships. Environment and Planning A, 43(12): 2992-3010.

Patel CJ, Bhattacharya J, Butte AJ. 2010. An Environment-Wide Association Study (EWAS) on Type 2 Diabetes Mellitus. PLoS ONE 5(5):e10746.

Patel CJ, Cullen MR, Ioannidis JP, Butte AJ. 2012. Systematic evaluation of environmental factors: persistent pollutants and nutrients correlated with serum lipid levels. Int $\mathbf{J}$ Epidemiol 41(3):828-843.

Porta M, Gasull M, Pumarega J. 2012. Commentary: A step towards more comprehensive analyses of life course effects of mixtures of environmental factors. Int J Epidemiol 41(3):843846.

Pronk A, Nuckols JR, De Roos AJ, Airola M, Colt JS, Cerhan JR, et al. 2013. Residential proximity to industrial combustion facilities and risk of non-Hodgkin lymphoma: A case-control study. Environ Health 12(20).

Rsolnp: General Non-linear Optimization Using Augmented Lagrange Multiplier Method [computer program]. Version R package version 1.142012.

Rudel RA, Seryak LM, Brody JG. 2008. PCB-containing wood floor finish is a likely source of elevated PCBs in residents' blood, household air and dust: A case study of exposure. Environ Health 7(2).

Shen M, Cozen W, Huang L, Colt J, De Roos AJ, Severson RK, et al. 2008. Census and geographic differences between respondents and nonrespondents in a case-control study of nonHodgkin lymphoma. Am J Epidemiol 167(3):350-361. 
Stern AH, Fagliano JA, Savrin JE, Freeman NC, Lioy PJ. 1998. The association of chromium in household dust with urinary chromium in residences adjacent to chromate production waste sites. Environ Health Perspect 106(12):833-839.

Tibshirani R. Regression shrinkage and selection via the lasso. Journal of the Royal Statistical Society. 1996;58(1):267-288.

Tu Y-K, Gunnell D, Gilthorpe MS. Simpson's Paradox, Lord's Paradox, and Suppression Effects are the same phenomenon - the reversal paradox. Emerging Themes in Epidemiology 2008;5:2.

Wheeler D. 2007. Diagnostic tools and a remedial method for collinearity in geographically weighted regression. Environment and Planning A, 39(10).

Wheeler D. 2014. Geographically weighted regression. In Handbook of Regional Science, Eds. M. Fischer and P. Nijkamp, Springer.

Wheeler D. 2009. Simultaneous coefficient penalization and model selection in geographically weighted regression: The geographically weighted lasso. Environment and Planning A, 41: 722742 .

Wheeler D, Calder C. 2007. An assessment of coefficient accuracy in linear regression models with spatially varying coefficients. Journal of Geographical Systems, 9(2).

Wheeler D, Tiefelsdorf M. 2005. Multicollinearity and correlation among local regression coefficients in geographically weighted regression. Journal of Geographical Systems, 7(2): 1-28.

Wheeler DC, De Roos AJ, Cerhan JR, Morton LM, Severson R, Cozen W, et al. 2011. Spatialtemporal analysis of non-Hodgkin lymphoma in the NCI-SEER NHL case-control study. Environ Health 10(63).

Whitehead T, Metayer C, Gunier R, et al. Determinants of polycyclic aromatic hydrocarbon levels in house dust. Journal of Exposure Science and Environmental Epidemiology. 2009:1-10.

Whitehead T, Metayer C, Ward M, et al. Persistent organic pollutants in dust from older homes: learning from lead. American Journal of Public Health. 2014.

Zou H. The adaptive lasso and its oracle properties. Journal of the American Statistical Association. 2006;101:1418-1429.

Zou H, Hastie T. Regularization and variable selection via the elastic net. Journal of the Royal Statistical Society.Series B (Statistical Methodology). 2005;67(2):301-320. 


\title{
Appendix A
}

\author{
Supplemental Material: Chapter 2
}

Table A1: Association between individual chemicals in carpet dust and non-Hodgkin lymphoma for all study sites combined.

\begin{tabular}{|c|c|c|}
\hline Chemical & Odds Ratio $^{\mathrm{a}}(95 \% \mathrm{CI})$ & $p$-value \\
\hline PCB 105 & $1.20(0.87,1.67)$ & 0.27 \\
\hline PCB 138 & $1.20(0.86,1.68)$ & 0.29 \\
\hline PCB 153 & $1.31(0.93,1.84)$ & 0.12 \\
\hline PCB 170 & $1.37(0.98,1.91)$ & 0.07 \\
\hline PCB 180 & $1.55(1.11,2.17)$ & 0.01 \\
\hline benz(a)anthracene & $0.86(0.57,1.29)$ & 0.47 \\
\hline benzo(b)fluoranthene & $0.83(0.55,1.25)$ & 0.37 \\
\hline benzo(k)fluoranthene & $0.96(0.64,1.43)$ & 0.83 \\
\hline benzo(a)pyrene & $0.96(0.64,1.44)$ & 0.84 \\
\hline chrysene & $0.79(0.53,1.17)$ & 0.24 \\
\hline dibenz(ah)anthracene & $0.91(0.61,1.37)$ & 0.66 \\
\hline indeno $(1,2,3-c d)$ pyrene & $0.79(0.52,1.19)$ & 0.26 \\
\hline$\alpha$-chlordane & $1.40(0.99,1.98)$ & 0.06 \\
\hline$\gamma$-chlordane & $1.35(0.95,1.92)$ & 0.09 \\
\hline carbaryl & $1.07(0.77,1.49)$ & 0.69 \\
\hline chlorpyrifos & $0.73(0.52,1.02)$ & 0.06 \\
\hline cis-permethrin & $0.95(0.67,1.34)$ & 0.76 \\
\hline trans-permethrin & $0.98(0.69,1.39)$ & 0.90 \\
\hline $2,4-\mathrm{D}$ & $0.70(0.48,1.03)$ & 0.07 \\
\hline DDE & $1.26(0.90,1.76)$ & 0.19 \\
\hline DDT & $1.03(0.73,1.44)$ & 0.87 \\
\hline diazinon & $0.79(0.56,1.10)$ & 0.16 \\
\hline dicamba & $0.74(0.53,1.04)$ & 0.08 \\
\hline methoxychlor & $0.90(0.64,1.27)$ & 0.55 \\
\hline$o$-phenylphenol & $1.00(0.71,1.41)$ & 0.99 \\
\hline pentachlorophenol & $1.02(0.72,1.45)$ & 0.92 \\
\hline propoxur & $1.27(0.90,1.79)$ & 0.18 \\
\hline
\end{tabular}

${ }^{a}$ Estimated odds ratios compare the fourth vs. first exposure quartile; quartile cut points were based on the distribution of cases and controls combined. Models were adjusted for gender, race, education, age, and study site. 
Table A2: Association between individual chemicals in carpet dust and non-Hodgkin lymphoma by study site.

\begin{tabular}{|c|c|c|c|c|c|c|c|c|}
\hline \multirow[b]{2}{*}{ Chemical } & \multicolumn{2}{|l|}{ Detroit } & \multicolumn{2}{|l|}{ Iowa } & \multicolumn{2}{|l|}{ Los Angeles } & \multicolumn{2}{|l|}{ Seattle } \\
\hline & $\mathrm{OR}^{\mathrm{a}}(95 \% \mathrm{CI})$ & $p$ & $\mathrm{OR}^{\mathrm{a}}(95 \% \mathrm{CI})$ & $p$ & $\mathrm{OR}^{\mathrm{a}}(95 \% \mathrm{CI})$ & $p$ & $\mathrm{OR}^{\mathrm{a}}(95 \% \mathrm{CI})$ & $p$ \\
\hline PCB 105 & $1.41(0.61,3.24)$ & 0.42 & $1.25(0.67,2.32)$ & 0.49 & $1.20(0.62,2.35)$ & 0.59 & $1.13(0.62,2.08)$ & 0.69 \\
\hline PCB 138 & $1.14(0.49,2.68)$ & 0.76 & $1.24(0.67,2.32)$ & 0.49 & $1.27(0.65,2.48)$ & 0.48 & $1.17(0.64,2.16)$ & 0.61 \\
\hline PCB 153 & $1.27(0.54,3.01)$ & 0.59 & $1.24(0.66,2.32)$ & 0.50 & $1.61(0.82,3.16)$ & 0.17 & $1.25(0.67,2.31)$ & 0.48 \\
\hline PCB 170 & $2.27(0.95,5.39)$ & 0.06 & $1.07(0.58,2.00)$ & 0.82 & $1.27(0.65,2.48)$ & 0.49 & $1.17(0.63,2.15)$ & 0.63 \\
\hline PCB 180 & $2.87(1.19,6.91)$ & 0.02 & $1.23(0.65,2.32)$ & 0.52 & $1.21(0.62,2.36)$ & 0.58 & $1.53(0.82,2.85)$ & 0.18 \\
\hline benz(a)anthracene & $0.64(0.26,1.58)$ & 0.33 & $0.86(0.46,1.59)$ & 0.63 & $0.88(0.46,1.70)$ & 0.71 & $1.13(0.61,2.06)$ & 0.70 \\
\hline benzo(b)fluoranthene & $0.73(0.30,1.76)$ & 0.48 & $0.89(0.48,1.65)$ & 0.72 & $1.66(0.85,3.25)$ & 0.13 & $0.93(0.51,1.71)$ & 0.81 \\
\hline benzo(k)fluoranthene & $0.79(0.33,1.91)$ & 0.60 & $0.94(0.51,1.73)$ & 0.83 & $2.05(1.04,4.04)$ & 0.04 & $1.11(0.61,2.03)$ & 0.73 \\
\hline benzo(a)pyrene & $0.88(0.37,2.13)$ & 0.78 & $1.05(0.57,1.94)$ & 0.88 & $0.84(0.43,1.65)$ & 0.62 & $1.00(0.54,1.83)$ & 0.99 \\
\hline Chrysene & $0.71(0.29,1.70)$ & 0.44 & $0.88(0.48,1.62)$ & 0.68 & $1.04(0.54,2.00)$ & 0.91 & $1.22(0.66,2.23)$ & 0.53 \\
\hline dibenz(ah)anthracene & $0.74(0.31,1.79)$ & 0.51 & $1.17(0.64,2.17)$ & 0.61 & $1.27(0.65,2.47)$ & 0.48 & $1.09(0.59,1.98)$ & 0.79 \\
\hline indeno( $(1,2,3-c d)$-pyrene & $0.90(0.37,2.18)$ & 0.82 & $1.18(0.63,2.18)$ & 0.60 & $0.93(0.48,1.82)$ & 0.84 & $1.01(0.55,1.85)$ & 0.97 \\
\hline$\alpha$-chlordane & $1.21(0.53,2.76)$ & 0.65 & $2.18(1.15,4.14)$ & 0.02 & $1.12(0.58,2.18)$ & 0.73 & $0.92(0.50,1.68)$ & 0.78 \\
\hline$\gamma$-chlordane & $0.84(0.37,1.94)$ & 0.69 & $2.25(1.20,4.24)$ & 0.01 & $1.26(0.65,2.45)$ & 0.49 & $0.89(0.49,1.63)$ & 0.71 \\
\hline Carbaryl & $1.59(0.68,3.74)$ & 0.28 & $0.72(0.38,1.33)$ & 0.29 & $1.16(0.60,2.23)$ & 0.66 & $1.05(0.57,1.93)$ & 0.88 \\
\hline Chlorpyrifos & $0.82(0.35,1.93)$ & 0.65 & $1.11(0.60,2.03)$ & 0.74 & $0.69(0.36,1.34)$ & 0.28 & $0.69(0.38,1.27)$ & 0.23 \\
\hline cis-permethrin & $1.60(0.69,3.72)$ & 0.28 & $0.74(0.40,1.36)$ & 0.33 & $1.10(0.57,2.11)$ & 0.79 & $1.06(0.58,1.95)$ & 0.85 \\
\hline trans-permethrin & $1.19(0.51,2.78)$ & 0.69 & $0.62(0.33,1.15)$ & 0.13 & $0.86(0.44,1.65)$ & 0.64 & $1.07(0.58,1.99)$ & 0.82 \\
\hline $2,4-\mathrm{D}$ & $1.11(0.47,2.66)$ & 0.81 & $0.36(0.19,0.68)$ & $<0.01$ & $1.05(0.54,2.04)$ & 0.89 & $0.53(0.29,0.97)$ & 0.04 \\
\hline DDE & $0.82(0.35,1.94)$ & 0.65 & $1.96(1.05,3.68)$ & 0.04 & $1.45(0.75,2.82)$ & 0.27 & $1.53(0.83,2.84)$ & 0.17 \\
\hline DDT & $0.97(0.41,2.28)$ & 0.95 & $1.06(0.57,1.97)$ & 0.86 & $1.13(0.58,2.17)$ & 0.72 & $1.19(0.64,2.24)$ & 0.58 \\
\hline Diazinon & $0.84(0.37,1.92)$ & 0.67 & $0.82(0.44,1.52)$ & 0.53 & $0.53(0.27,1.04)$ & 0.07 & $0.81(0.44,1.48)$ & 0.49 \\
\hline Dicamba & $1.07(0.45,2.54)$ & 0.88 & $0.48(0.26,0.90)$ & 0.02 & $0.93(0.48,1.81)$ & 0.83 & $0.41(0.22,0.76)$ & $<0.01$ \\
\hline Methoxychlor & $1.92(0.81,4.57)$ & 0.14 & $0.98(0.53,1.82)$ & 0.95 & $0.68(0.35,1.33)$ & 0.26 & $0.62(0.33,1.15)$ & 0.13 \\
\hline$o$-phenylphenol & $0.52(0.22,1.27)$ & 0.15 & $1.58(0.83,3.00)$ & 0.16 & $0.52(0.26,1.02)$ & 0.06 & $1.03(0.56,1.89)$ & 0.93 \\
\hline Pentachlorophenol & $0.66(0.27,1.62)$ & 0.36 & $1.24(0.67,2.30)$ & 0.50 & $0.76(0.39,1.48)$ & 0.42 & $1.45(0.78,2.73)$ & 0.24 \\
\hline Propoxur & $1.06(0.45,2.52)$ & 0.89 & $2.02(1.09,3.78)$ & 0.03 & $0.60(0.30,1.17)$ & 0.13 & $1.53(0.82,2.85)$ & 0.18 \\
\hline
\end{tabular}

Abbreviations: OR, odds ratio; $p, p$-value

${ }^{\text {a }}$ Estimated odds ratios compare the fourth vs. first exposure quartile based on site-specific cut points of cases and controls combined. Models were adjusted for gender, race, education, and age. 


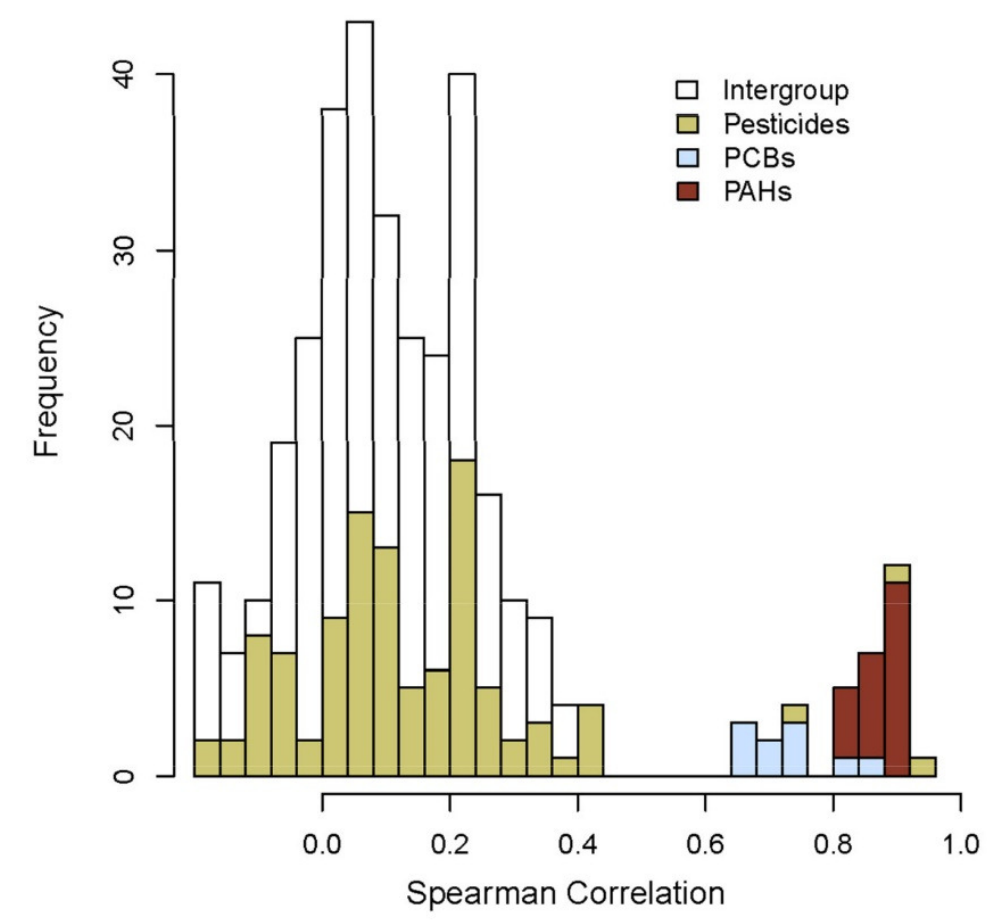

Figure A1: Pairwise correlations among the 27 chemical concentrations by type of chemical. There is a high level of correlation within PCBs and PAHs, while the correlations among pesticides and the correlations between chemical groups (intergroup) are lower. 

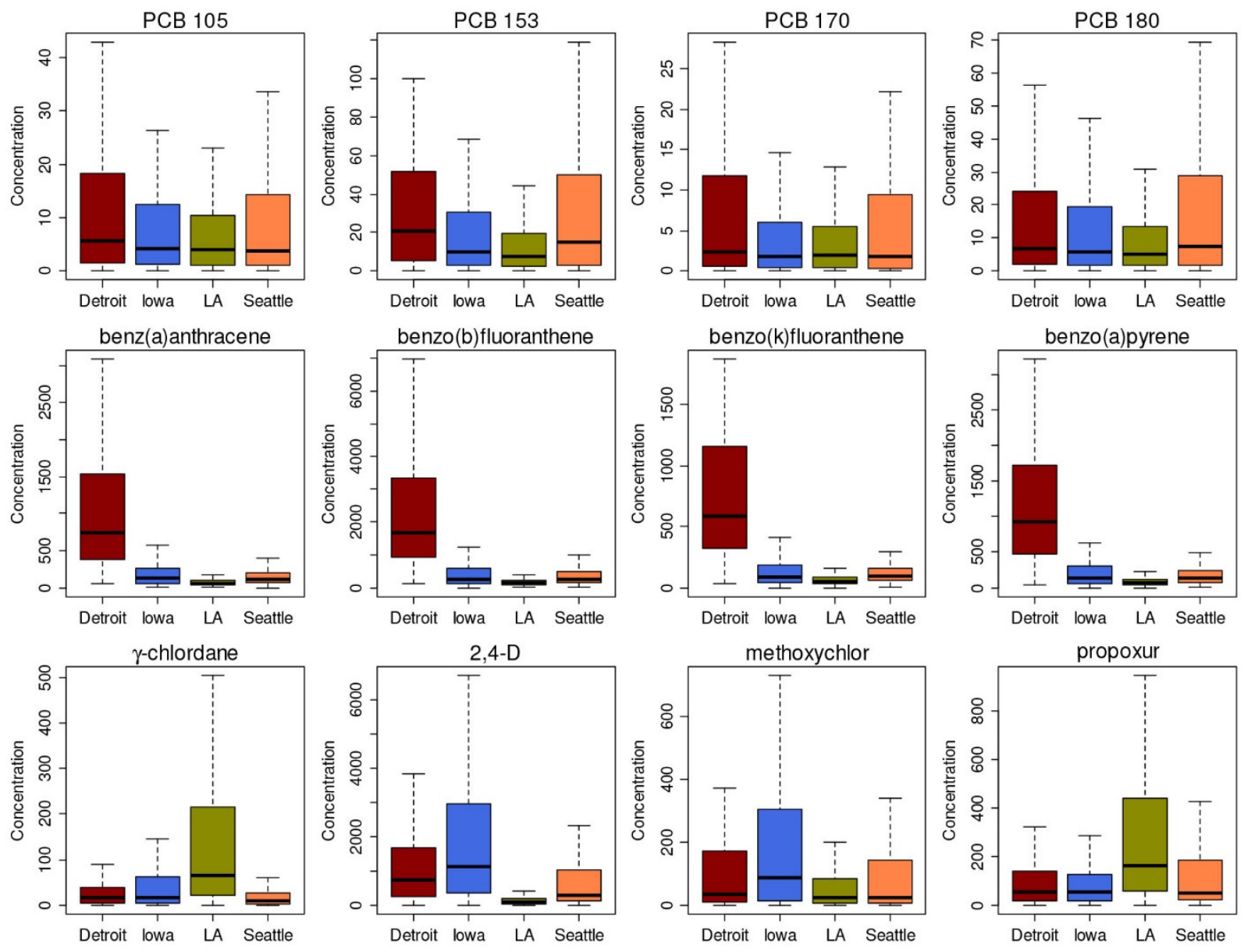

Figure A2: Distribution of chemical concentrations among cases and controls combined in carpet dust by study site for selected chemicals. Boxes extend from the 25 th to the 75 th percentile, horizontal bars represent the median, and whiskers extend 1.5 times the length of the interquartile range (IQR) above and below the 75th and 25th percentiles, respectively. 

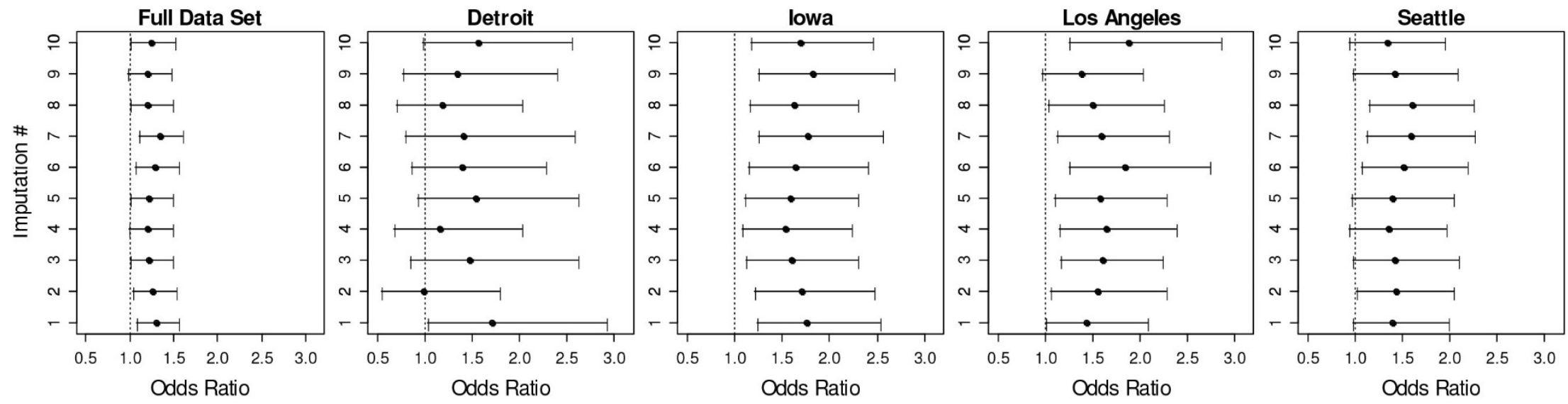

Figure A3: Associations ${ }^{a}$ between non-Hodgkin lymphoma and weighted quantile sum regression index across the ten imputations for the study population and each study site.

${ }^{\text {a }}$ Estimated odds ratio and 95\% confidence interval (displayed as error bars) associated with a unit increase in the weighted quantile sum regression index. All models were adjusted for gender, race, education, and age. The model for the study population (i.e., the full data set) was also adjusted for study site. 

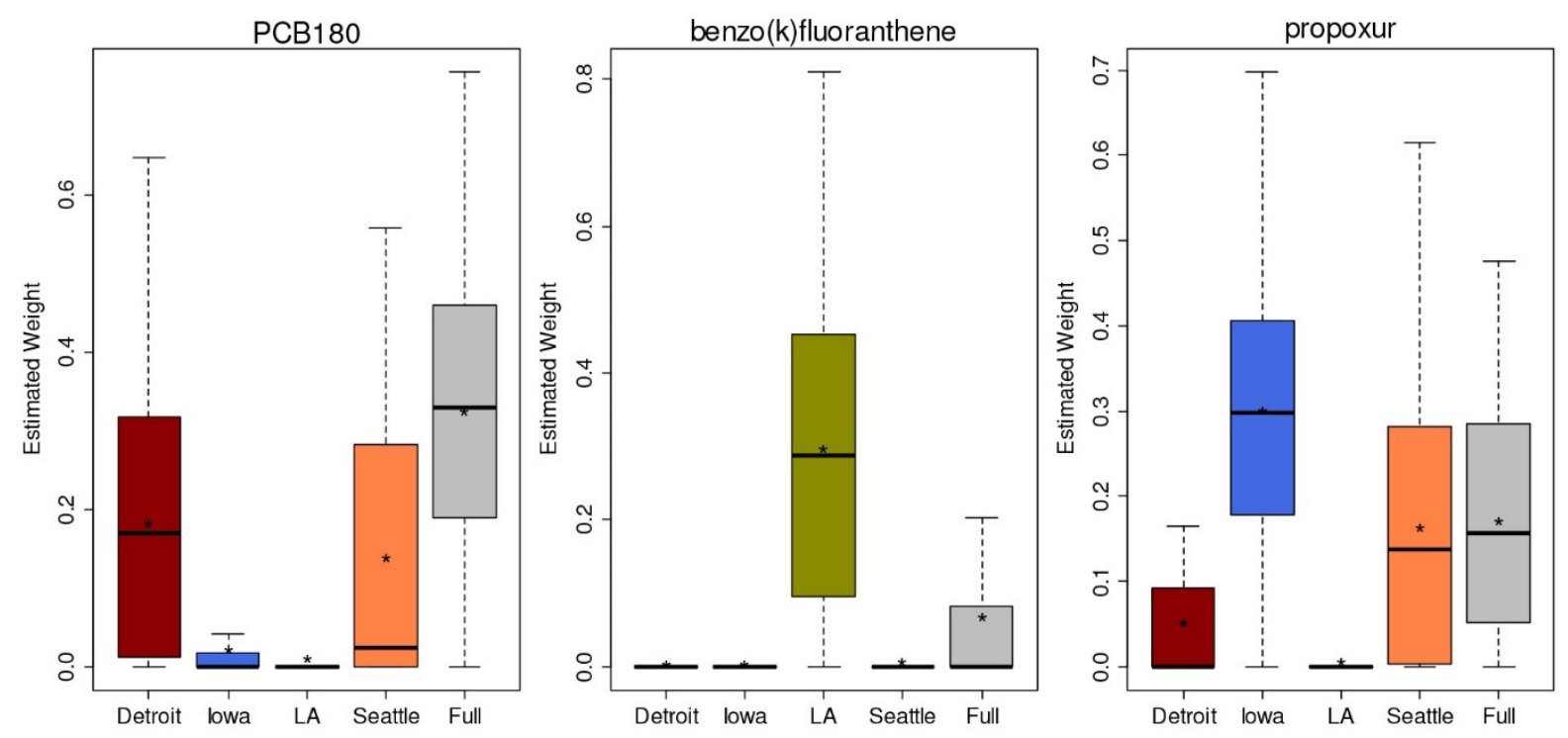

Figure A4: Distribution of estimated weights for selected chemicals from the weighted quantile sum regression model of non-Hodgkin lymphoma in the study population and each study site. Boxes extend from the 25th to the 75th percentile, horizontal bars represent the median, and whiskers extend 1.5 times the length of the interquartile range (IQR) above and below the 75th and 25 th percentiles, respectively. The asterisk denotes the mean weight. 


\section{Appendix B}

Supplemental Material: Chapter 5 
Table B1: Summary of simulation settings by scenario.

Scenario 1: Mixture effect and weight parameters constant over space; No systematic spatial variation in exposures.

\begin{tabular}{|c|c|c|c|}
\hline Mixture Effect & Weight parameters & Exposure Data & Correlation Case \\
\hline \multirow{4}{*}{$\begin{array}{l}\text { No spatial variation } \\
\text { b1 }=1\end{array}$} & \multirow{4}{*}{$\begin{array}{l}\text { No spatial variation } \\
\mathrm{w}=(0.3,0,0.1,0,0,0.6)\end{array}$} & \multirow{4}{*}{$\begin{array}{l}\text { Flat exposures } \\
\text { Exposures for } x 1, \ldots, \mathrm{x} 6 \\
\text { are simulated as multivariate } \\
\text { normal with standard deviations } \\
\text { of } 0.5 \text { and mean vector } \\
\mu_{\mathrm{x}}=(5,1.5,2.5,4,3,6)\end{array}$} & a) Independent Exposures \\
\hline & & & b) $r(x 1, x 3)=0.6$ \\
\hline & & & c) Correlation Structure 1 \\
\hline & & & $\begin{array}{rrrrrrr} & \mathrm{x} 1 & \mathrm{x} 2 & \mathrm{x} 3 & \mathrm{x} 4 & \mathrm{x} 5 & \mathrm{x} 6 \\
\mathrm{x} 1 & 1.00 & 0.71 & 0.77 & 0.24 & 0.29 & 0.37 \\
\mathrm{x} 2 & & 1.00 & 0.84 & 0.11 & 0.14 & 0.26 \\
\mathrm{x} 3 & & & 1.00 & 0.15 & 0.20 & 0.31 \\
\mathrm{x} 4 & & & & 1.00 & 0.91 & 0.08 \\
\mathrm{x} 5 & & & & & 1.00 & 0.16 \\
\mathrm{x} 6 & & & & & & 1.00\end{array}$ \\
\hline
\end{tabular}

Scenario 2: Mixture effect constant over space; Weight parameters change by row; No systematic spatial variation in exposures.

\begin{tabular}{|c|c|c|c|}
\hline \multirow{3}{*}{$\begin{array}{l}\text { No spatial variation } \\
\text { b1 }=1\end{array}$} & \multirow{3}{*}{$\begin{array}{l}\text { Weights change by row } \\
\text { Row 1: } \mathrm{w}=(0.5,0,0.1,0,0,0.4) \\
\text { Row 2: } \mathrm{w}=(0.3,0,0.1,0,0,0.6) \\
\text { Row 3: } \mathrm{w}=(0.1,0,0.1,0,0,0.8)\end{array}$} & \multirow{3}{*}{$\begin{array}{l}\text { Flat exposures } \\
\mu_{x}=(5,1.5,2.5,4,3,6) \\
\mathrm{sd}=0.5\end{array}$} & a) Independent Exposures \\
\hline & & & b) $r(x 1, x 3)=0.6$ \\
\hline & & & \begin{tabular}{lrrrrrr}
\multicolumn{6}{c}{ c) Correlation Structure 2 } \\
& $\mathrm{x} 1$ & $\mathrm{x} 2$ & $\mathrm{x} 3$ & $\mathrm{x} 4$ & $\mathrm{x} 5$ & $\mathrm{x} 6$ \\
$\mathrm{x} 1$ & 1.00 & 0.71 & 0.77 & 0.37 & 0.24 & 0.29 \\
$\mathrm{x} 2$ & & 1.00 & 0.84 & 0.26 & 0.11 & 0.14 \\
$\mathrm{x} 3$ & & & 1.00 & 0.31 & 0.15 & 0.20 \\
$\mathrm{x} 4$ & & & & 1.00 & 0.08 & 0.16 \\
$\mathrm{x} 5$ & & & & & 1.00 & 0.91 \\
$\mathrm{x} 6$ & & & & & & 1.00
\end{tabular} \\
\hline
\end{tabular}




\begin{tabular}{|c|c|c|c|}
\hline \multicolumn{4}{|c|}{ Scenario 3: Mixture increases by row; Weight parameters change by row; No systematic spatial variation in exposures. } \\
\hline \multirow{16}{*}{$\begin{array}{l}\text { b1 increases by row } \\
\text { from row } 1 \text { to row } 3 \\
\text { b1 }=0.5 \\
\text { b1 }=0.7 \\
\text { b1 }=1.0\end{array}$} & \multirow{16}{*}{$\begin{array}{l}\text { Weights change by row } \\
\text { Row 1: } \mathrm{w}=(0.5,0,0.1,0,0,0.4) \\
\text { Row 2: } \mathrm{w}=(0.3,0,0.1,0,0,0.6) \\
\text { Row 3: } \mathrm{w}=(0.1,0,0.1,0,0,0.8)\end{array}$} & \multirow{16}{*}{$\begin{array}{l}\text { Flat exposures } \\
\mu_{\mathrm{x}}=(5,1.5,2.5,4,3,6) \\
\mathrm{sd}=0.5\end{array}$} & \multirow{8}{*}{\begin{tabular}{lrrrrrr}
\multicolumn{6}{l}{ a) Correlation Structure 2 } \\
& $\mathrm{x} 1$ & $\mathrm{x} 2$ & $\mathrm{x} 3$ & $\mathrm{x} 4$ & $\mathrm{x} 5$ & $\mathrm{x} 6$ \\
$\mathrm{x} 1$ & 1.00 & 0.71 & 0.77 & 0.37 & 0.24 & 0.29 \\
$\mathrm{x} 2$ & & 1.00 & 0.84 & 0.26 & 0.11 & 0.14 \\
$\mathrm{x} 3$ & & & 1.00 & 0.31 & 0.15 & 0.20 \\
x4 & & & & 1.00 & 0.08 & 0.16 \\
x5 & & & & 1.00 & 0.91 \\
x6 & & & & & 1.00
\end{tabular}} \\
\hline & & & \\
\hline & & & \\
\hline & & & \\
\hline & & & \\
\hline & & & \\
\hline & & & \\
\hline & & & \\
\hline & & & \multirow{8}{*}{$\begin{array}{l}\text { b) Correlation Structure 3 } \\
\text { 6 PAHs (all highly correlated) } \\
\begin{array}{lrrrrrr} \\
\text { x1 } & \text { x2 } & \text { x3 } & \text { x4 } & \text { x5 } & \text { x6 } \\
\text { x1 } & 1.00 & 0.94 & 0.91 & 0.91 & 0.88 & 0.94 \\
\text { x2 } & & 1.00 & 0.94 & 0.95 & 0.88 & 0.94 \\
\text { x3 } & & 1.00 & 0.91 & 0.87 & 0.92 \\
\text { x4 } & & & 1.00 & 0.87 & 0.91 \\
\text { x5 } & & & & 1.00 & 0.89 \\
\text { x6 } & & & & & 1.00\end{array}\end{array}$} \\
\hline & & & \\
\hline & & & \\
\hline & & & \\
\hline & & & \\
\hline & & & \\
\hline & & & \\
\hline & & & \\
\hline \multicolumn{4}{|c|}{ Scenario 4: Mixture effect and exposures x2, x4, \& x6 decrease radially from center of grid; Weight parameters constant over space. } \\
\hline \multirow[t]{6}{*}{$\begin{array}{l}\text { Radial pattern } \\
\mathrm{b} 1=\exp (-2 \mathrm{~d})\end{array}$} & $\begin{array}{l}\text { No spatial variation } \\
\mathrm{w}=(0.3,0,0.1,0,0,0.6)\end{array}$ & $\begin{array}{l}\text { Radial pattern in } \mathbf{x} 2, \mathbf{x} 4, \text { and } x 6 \\
\text { Exposures for } x 1, x 3 \text {, and } x 5 \text { are }\end{array}$ & \multirow{6}{*}{$\begin{array}{l}\text { Based on correlation structure } 3: \\
\mathrm{r}(\mathrm{x} 1, \mathrm{x} 3)=0.91 \\
\mathrm{r}(\mathrm{x} 1, \mathrm{x} 5)=0.88 \\
\mathrm{r}(\mathrm{x} 3, \mathrm{x} 5)=0.87\end{array}$} \\
\hline & & simulated as multivariate normal & \\
\hline & & with means $\mu_{\mathrm{x}}=(5.0,2.5,3.0)$ & \\
\hline & & and standard deviations of 0.5 . & \\
\hline & & Exposures for $\mathrm{x} 2, \mathrm{x} 4$, and $\mathrm{x} 6$ were & \\
\hline & & simulated as & \\
\hline
\end{tabular}




\begin{tabular}{|c|c|c|c|}
\hline & & $\begin{array}{l}x_{6}=3+5 \exp (-2 d)+\varepsilon_{N(0,0.5)} \\
x_{2}=x_{6}-\varepsilon_{N(1,1)} \\
x_{4}=\sqrt{x_{6}}+\varepsilon_{N(0,0.5)}\end{array}$ & \\
\hline \multicolumn{4}{|c|}{ Scenario 5: Mixture effect, exposures x2, x4, \& x6, and weight parameter w6 decrease radially from the center of grid. } \\
\hline $\begin{array}{l}\text { Radial pattern } \\
\mathrm{b} 1=\exp (-2 \mathrm{~d})\end{array}$ & $\begin{array}{l}\text { Radial pattern in w6 } \\
\text { w6 }=\exp (-\mathrm{d})-0.3 \\
\mathrm{w} 1=\mathrm{k}(1-\mathrm{w} 6) \\
\mathrm{w} 3=(1-\mathrm{k})(1-\mathrm{w} 6) \\
k \in[0.3,0.7]\end{array}$ & $\begin{array}{l}\text { Radial pattern in } \mathbf{x} 2, \mathbf{x} 4, \text { and } \mathbf{x} 6 \\
\text { Exposures for } \mathrm{x} 1, \mathrm{x} 3 \text {, and } \mathrm{x} 5 \text { are } \\
\text { simulated as multivariate normal } \\
\text { with means } \mu_{\mathrm{x}}=(5.0,2.5,3.0) \\
\text { and standard deviations of } 0.5 \text {. } \\
\text { Exposures for } \mathrm{x} 2, \mathrm{x} 4 \text {, and } \mathrm{x} 6 \text { were } \\
\text { simulated as } \\
x_{6}=3+5 \exp (-2 d)+\varepsilon_{N(0,0.5)} \\
x_{2}=x_{6}-\varepsilon_{N(1,1)} \\
x_{4}=\sqrt{x_{6}}+\varepsilon_{N(0,0.5)}\end{array}$ & $\begin{array}{l}\text { Based on correlation structure 3: } \\
r(x 1, x 3)=0.91 \\
r(x 1, x 5)=0.88 \\
r(x 3, x 5)=0.87\end{array}$ \\
\hline
\end{tabular}

$d=\left(d_{1}, \ldots, d_{\mathrm{N}}\right)$ is the vector of distances from the center of the study region to the $i=1, \ldots, \mathrm{N}$ locations with the $i^{\text {th }}$ entry defined as $d_{i}=\sqrt{\left(u_{i}-0.5\right)^{2}+\left(v_{i}-0.5\right)^{2}}$, where $\left(u_{i}, v_{i}\right)$ are the spatial coordinates for location $i$.

Note: The continuous outcome variable, y, was simulated through the WQS model $y_{i}=\beta_{0}+\beta_{1 i} \sum_{j=1}^{6} \omega_{i j} q_{i j}+\varepsilon_{i}$, where $\varepsilon_{i} \sim \mathrm{N}(0,0.1)$.

In all scenarios, exposure variables $\mathrm{x} 1, \mathrm{x} 3$, and $\mathrm{x} 6$ are related to the response variable through the mixture effect and weight parameters. Exposure variables $\mathrm{x} 2, \mathrm{x} 4$, and $\mathrm{x} 5$ are assumed to have no relationship with the outcome, and therefore the weight parameters for these variables are set to zero. 


\section{Vita}

Jenna Nichole Czarnota was born on January $13^{\text {th }}, 1988$, in Newark, Delaware. She graduated from Elkton High School in Cecil County, Maryland in 2005. Jenna received a Bachelor of Science in Mathematics with a minor in Economics from the University of Maryland Baltimore County (UMBC) in Catonsville, Maryland in 2010. Jenna was a Meyerhoff Scholar at UMBC from 2005 to 2010, and a National Institute of Environmental Health Sciences Research Service Award Trainee from 2013 to 2016 at Virginia Commonwealth University. 